\title{
ENDWALL FLOWS IN TRANSONIC TURBINE CASCADES
}

\author{
by
}

\section{Farzad Taremi}

A thesis submitted to the Faculty of Graduate and Postdoctoral Affairs in partial fulfillment of the requirements for the degree of

\section{Doctor of Philosophy}

in Aerospace Engineering

Ottawa-Carleton Institute for Mechanical and Aerospace Engineering

Department of Mechanical and Aerospace Engineering

Carleton University

Ottawa, Ontario, Canada

(C) Farzad Taremi, 2013 
The undersigned recommend to

the Faculty of Graduate Studies and Research acceptance of the thesis

\title{
Endwall Flows in Transonic Turbine Cascades
}

\author{
Submitted by \\ Farzad Taremi \\ in partial fulfilment of the requirements for the degree of \\ Doctor of Philosophy in Aerospace Engineering
}

Thesis Supervisor: Professor Steen. A. Sjolander, Department of Mechanical and Aerospace Engineering

Department Chair: Professor Metin I. Yaras, Department of Mechanical and Aerospace Engineering

External Examiner: Dr. Ali Mahallati, National Research Council of Canada

Carleton University

2013 
Dedicated to my mother. 


\section{Abstract}

The results of an investigation of the midspan and endwall flows in transonic linear turbine cascades are documented here. A family of five turbine cascades with different levels of flow turning and aerodynamic loading were used for this study to quantify the effects of these parameters on loss generation in compressible flows. Experimental data on these flows are scarce in the open literature. In addition, the application of two different passive flow control techniques for reducing the endwall losses is examined here: these are referred to as endwall contouring, and airfoil pressure-side modification.

The experimental investigations were conducted in the Pratt and Whitney Canada (PWC) High-Speed Wind Tunnel Laboratory at Carleton University. The measurements were made using a seven-hole pressure probe downstream of the cascades at both design and off-design Mach numbers. In addition to the measurements, surface flow visualization was conducted to assist in the interpretation of the flow physics. The results from complementary numerical investigations are also presented, and compared with the experimental data.

Overall, the examined secondary flow structures are in agreement with previous lowspeed findings. However, in contrast to low-speed results, the downstream mixing losses are mainly attributed to the dissipation of primary kinetic energy in the present study. Raising the exit Mach number results in weaker secondary flow structures and smaller secondary losses.

The experimental results demonstrate that endwall contouring is a viable option for mitigating the secondary flows, particularly for the more highly-loaded cascade. The modification of the airfoil pressure surface is also found to provide a significant benefit in terms of endwall loss reduction. The differences between the measurements and the computational results highlight the need for detailed experimental investigations. 


\section{Acknowledgments}

I would like to express my gratitude to my thesis supervisor, Professor Steen Sjolander, for giving me the opportunity to work on this research project. I would also like to thank him for his invaluable advice regarding the interpretation of the results, and for reviewing the text.

I would like to thank Dr. Thomas Praisner for his technical support and guidance, and for his efforts in getting permission for publishing the work.

The financial support of Pratt \& Whitney Aircraft, the Government of Ontario (through the Ontario Graduate Scholarship Program), and the Department of Mechanical and Aerospace Engineering (through the J.Y. and E.W. Wong Research Award in Mechanical/Aerospace Engineering) is gratefully acknowledged.

I would like to thank the following individuals for their technical contributions: Dr. Daniel Knezevici, Gordon MacIsaac, Steven Elia, Hamza Abo El Ella, Andrew Scribner, Steven Hall and Lanqin Yuan. I would also like to thank the staff of the Department of Mechanical and Aerospace Engineering.

Finally, I am grateful to my family and friends for their encouragement, patience and support. Thank you. 


\section{Table of Contents}

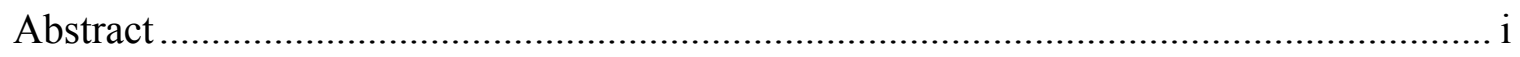

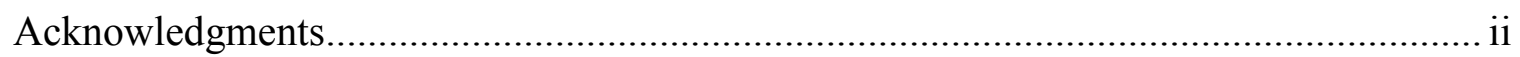

List of Tables .................................................................................................... ix

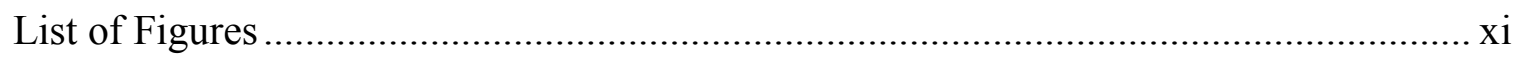

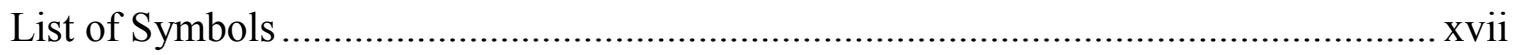

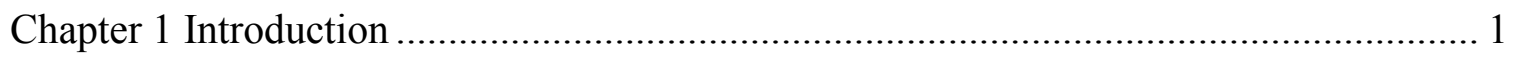

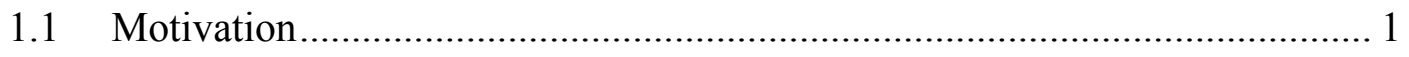

1.2 Research Objectives .......................................................................... 3

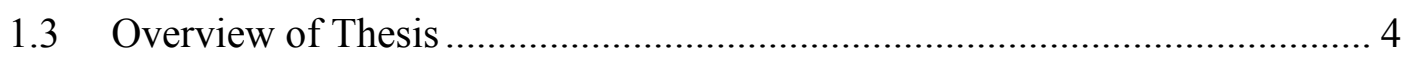

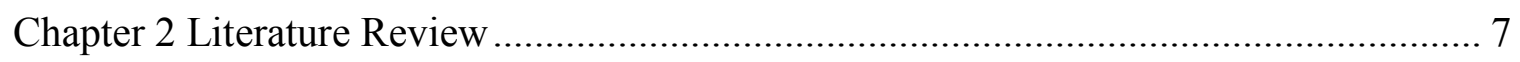

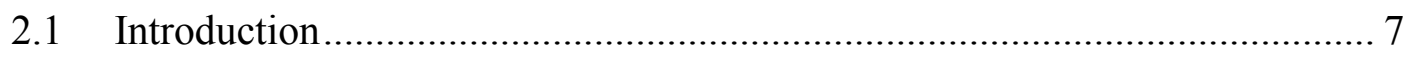

2.2 Flow Field and Losses in Turbine Blade Passages ................................... 7

2.3 Role of Linear Turbine Cascade Research................................................ 9

2.4 Characterization of Blade Loading .................................................. 11

2.5 Profile and Secondary Losses in Linear Turbine Cascades ........................ 13

2.5.1 Profile Losses........................................................................ 13

2.5.2 Secondary Flows and Secondary Losses ........................................ 13

2.6 Linear Turbine Cascade Research on Secondary Losses............................ 16

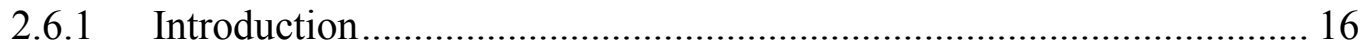

2.6.2 Inlet Boundary Layer Thickness and Free-Stream Turbulence ............ 18 
2.6.3 Zweifel Coefficient and Blade Loading Distribution ........................... 20

2.6.4 Total Flow Turning ...................................................................... 23

2.6.5 Flow Compressibility .................................................................. 24

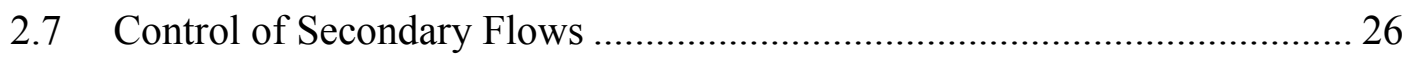

2.7.1 Introduction ................................................................................. 26

2.7.2 Endwall Contouring Research ......................................................... 28

2.7.3 Airfoil Pressure Surface Modification ................................................... 30

Chapter 3 Experimental Apparatus and Measurement Procedures..................................... 32

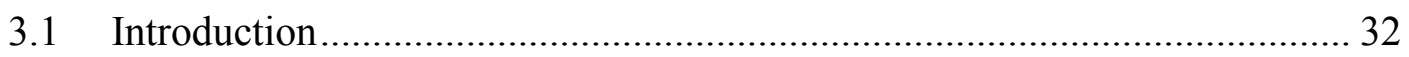

3.2 PWC High-Speed Wind Tunnel Laboratory ................................................... 32

3.2.1 Main Features of the Wind Tunnel ........................................................ 32

3.2.2 Wind Tunnel Test Section ................................................................. 34

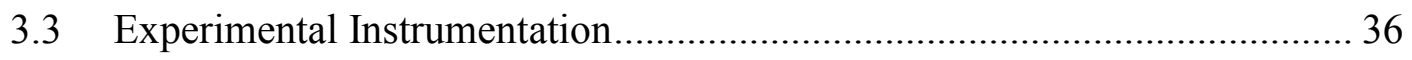

3.3.1 Data Acquisition System....................................................................... 36

3.3.2 Pressure and Temperature Probes ...................................................... 37

3.3.3 Probe Traverse Mechanism.................................................................. 38

3.3.4 Probe Calibration Rig ....................................................................... 39

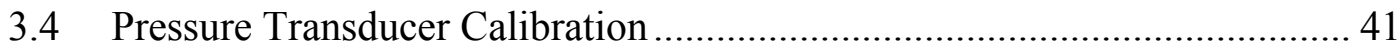

3.5 Kiel Probe Assessment: Flow Angle Sensitivity …………............................. 41

3.6 Seven-Hole Probe Calibration .................................................................. 42

3.7 Data Acquisition Procedures....................................................................... 44

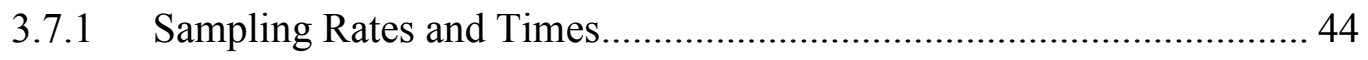

3.7.2 Inlet and Exit Measurements ……………………………………..... 45

3.7.3 Static Pressure Measurements............................................................. 46

3.8 Surface Flow Visualization Procedures ……………………………………... 47

3.9 Test Section Flow Quality Analysis ............................................................. 47

3.10 Turbine Cascade Geometries ...................................................................... 49

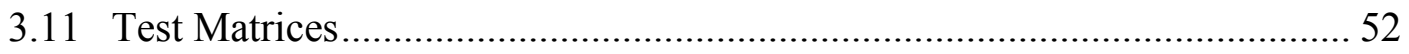


Chapter 4 Data Reduction Procedures and Uncertainty Analysis

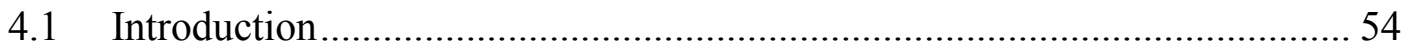

4.2 Loss Coefficients and Secondary Flow Parameters ................................... 54

4.2.1 Entropy Production and Loss Coefficients ...................................... 54

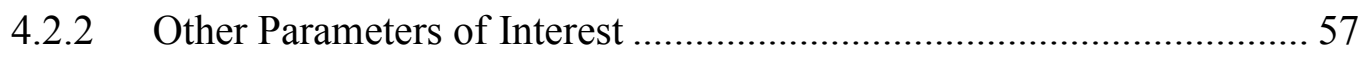

4.2.3 Reduction of the Data from the Measurement Plane .......................... 61

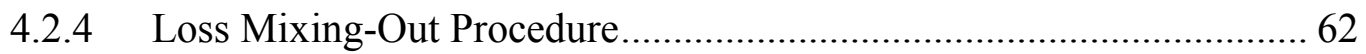

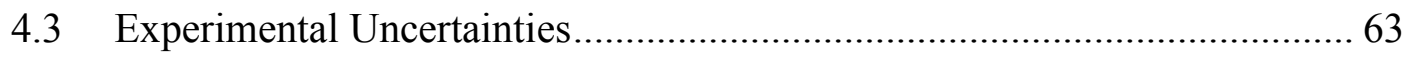

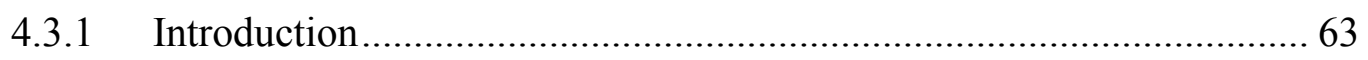

4.3.2 Sources of Uncertainty and Analysis Procedure............................... 64

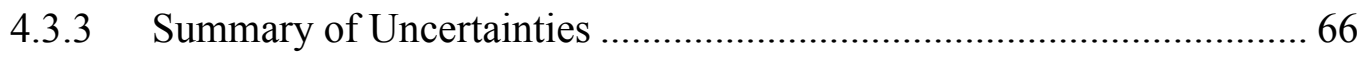

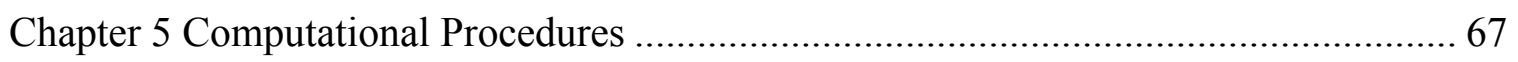

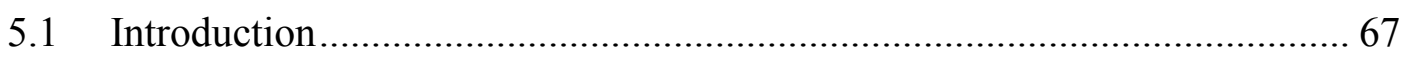

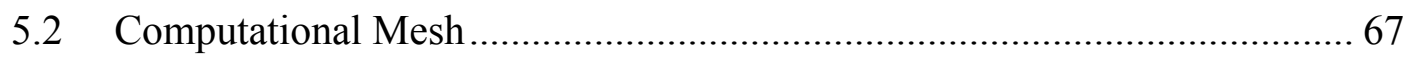

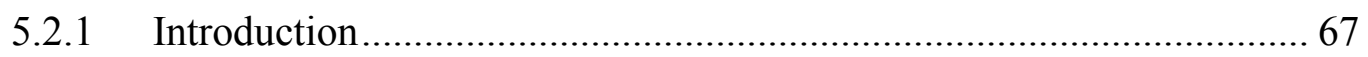

5.2.2 Inlet Pre-Domain: Inlet Endwall Boundary Layers ........................... 68

5.2.3 Turbine Cascades: 2D and 3D Mesh Generation............................... 68

5.3 Reynolds-Averaged Navier-Stokes Solver: ANSYS CFX ......................... 70

5.3.1 General Procedures .................................................................... 70

5.3.2 Simulation Settings and Boundary Conditions ................................ 72

5.4 Convergence Criteria and Convergence Analysis ................................... 74

5.4.1 2D Convergence Analysis......................................................... 74

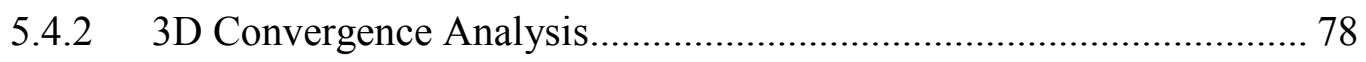

5.5 Sensitivity of 2D CFD Predictions to Inlet Turbulence and Transition

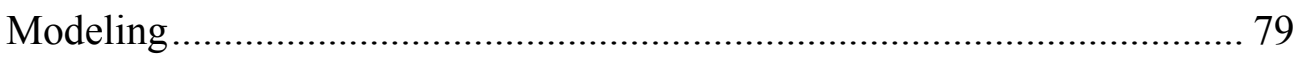

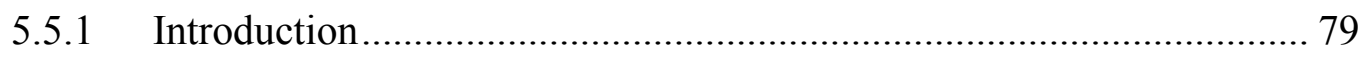

5.5.2 Sensitivity of 2D Predictions to Inlet Eddy Viscosity Ratio................. 80

5.5.3 Sensitivity of 2D Predictions to Inlet Turbulence Intensity ................. 82

Chapter 6 Midspan Airfoil Loading Distributions and Profile Losses ........................... 84 


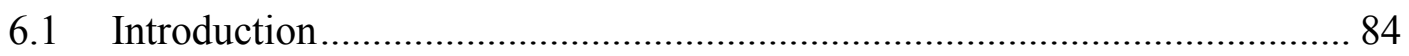

6.2 Summary of Cascade Parameters at Design and Off-Design Conditions..... 85

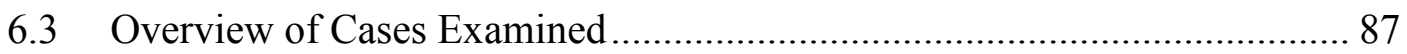

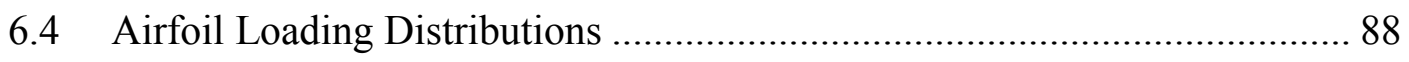

6.4.1 Experimental and Numerical Loading Results at the Design Mach

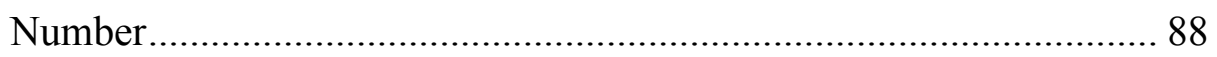

6.4.2 Experimental and Numerical Loading Results at Off-Design Mach

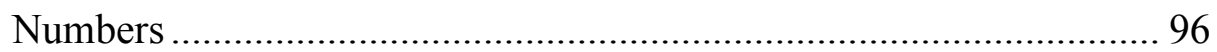

6.4.3 Additional Effects of Mach Number and Reynolds Number on

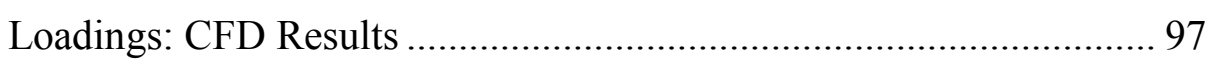

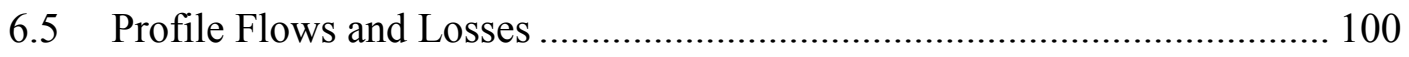

6.5.1 Experimental and Numerical Results at Tested Mach Numbers ........ 100

6.5.2 Additional Effects of Mach Number and Reynolds Number on Profile

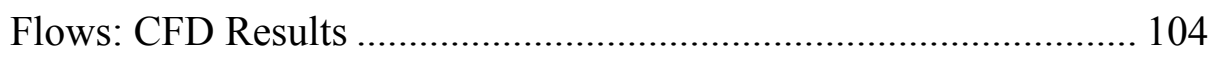

6.5.3 Comparisons with Kacker and Okapuu (1982) Loss Correlation....... 107 Chapter 7 Secondary Flows and Losses in Turbine Cascades with Flat Endwalls......... 111

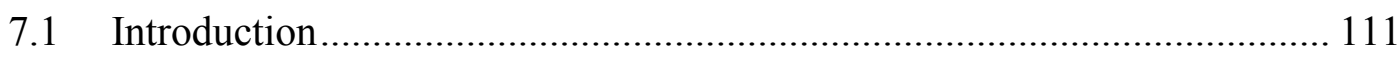

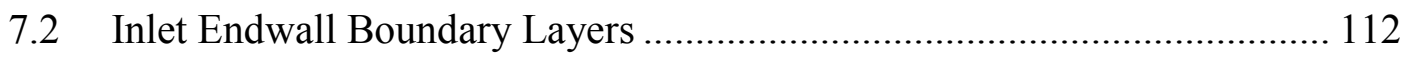

7.3 Results from the Low-Turning Cascades: SL3F and SL4F ........................ 113

7.3.1 Turbine Cascades .............................................................................. 113

7.3.2 Midspan Blade Loading Distributions ................................................. 114

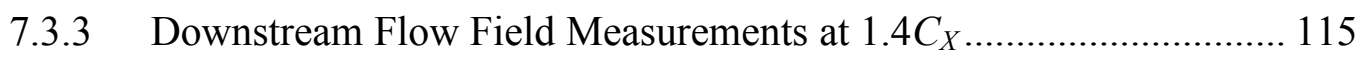

7.3.4 Downstream Growth of Secondary Losses .......................................... 122

7.4 Results from the High-Turning Cascades: SL1F and SL2F ……............... 126

7.4.1 Turbine Cascades ......................................................................... 126

7.4.2 Midspan Blade Loading Distributions ................................................. 126

7.4.3 Surface Flow Visualization Results at Design Conditions ................. 128

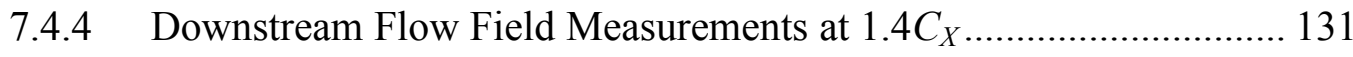

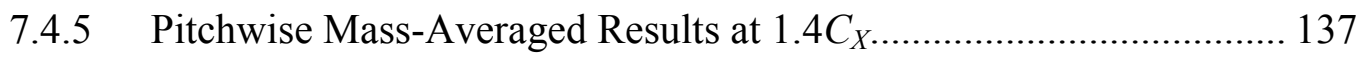


7.4.6 Downstream Growth of Secondary Losses at the Design Mach Number 139

7.4.7 CFD Predictions at the Design Mach Number .................................... 141

7.5 Overall Integrated Results: Mass Averaged and Mixed Out ....................... 146 Chapter 8 Secondary Flows and Losses in Turbine Cascades with Contoured Endwalls 150

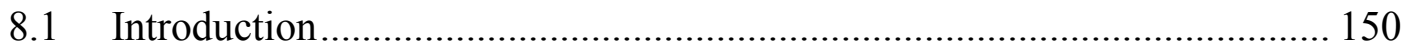

8.2 Non-Axisymmetric Endwall Contouring Design......................................... 151

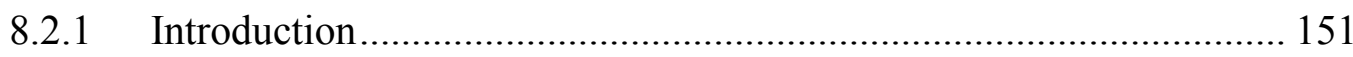

8.2.2 Computational Analysis for Design and Optimization ........................ 151

8.2.3 Geometric Features of the Endwalls ................................................... 153

8.2.4 Predicted Airfoil and Endwall Isentropic Mach Number Distributions 155

8.3 Experimental Results at the Design Mach Number .................................... 157

8.3.1 Surface Flow Visualization Results ................................................... 157

8.3.2 Downstream Flow Field Measurements at $1.4 C_{X} \ldots \ldots \ldots \ldots \ldots \ldots \ldots \ldots \ldots \ldots \ldots . . . . . . . . . . . . .160$

8.3.3 Downstream Growth of Secondary Losses ........................................... 163

8.4 Computational Results at the Design Mach Number................................... 166

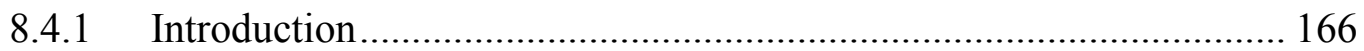

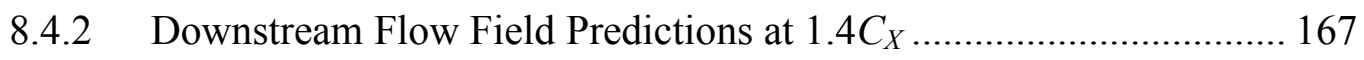

8.5 Integrated Results at both Design and Off-Design Mach Numbers ............ 170 Chapter 9 Modification of the Airfoil Pressure Surface for Secondary Loss Reduction 175

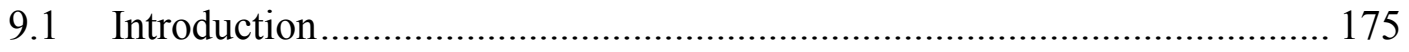

9.2 Concept for the Modified Pressure Side ........................................................ 175

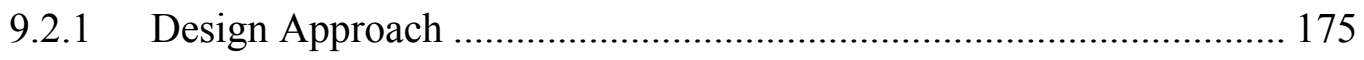

9.2.2 Predicted Airfoil and Endwall Isentropic Mach Number Distributions 177

9.3 Experimental Results at the Design Mach Number..................................... 178 
9.3.1 Surface Flow Visualizations ..................................................... 178

9.3.2 Downstream Flow Field Measurements at $1.4 C_{X} \ldots \ldots \ldots \ldots \ldots \ldots \ldots \ldots \ldots . . . . . . . . . . . .180$

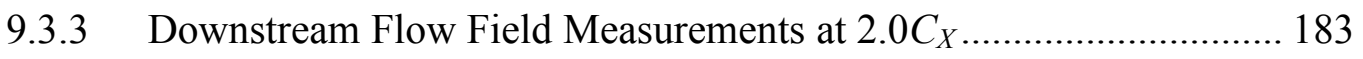

9.4 Computational Results at the Design Mach Number................................ 185

9.5 Integrated Results at both Design and Off-Design Mach Numbers .......... 187

Chapter 10 Summary, Conclusions and Recommendations .................................... 190

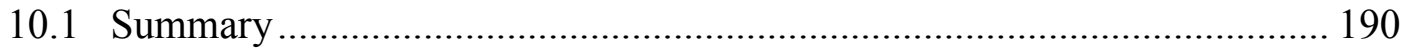

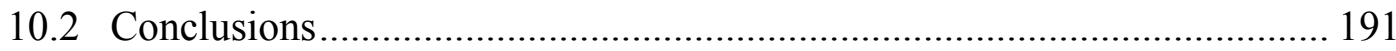

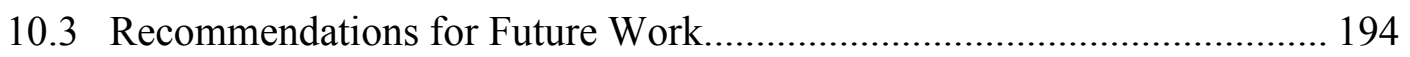

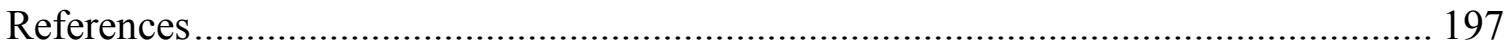

Appendix A Derivation of Streamwise Vorticity …............................................. 209

Appendix B Pitchwise Averaged Plots ................................................................. 212

Appendix C Loss Mixing Out Procedure............................................................... 214 


\section{List of Tables}

Table 1.1 Boeing 737 and Airbus A320 data (www.boeing.com and www.airbus.com) .. 2 Table 2.1 Technology Readiness Levels used by PWA (TRLP) ................................... 10

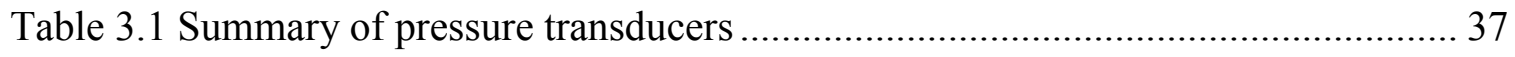

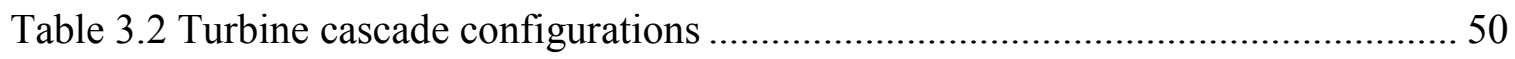

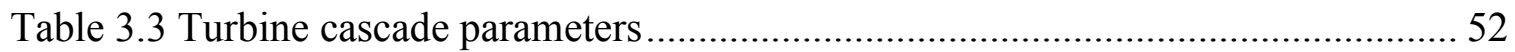

Table 3.4 Test cases for the low-turning cascades: SL3 and SL4 (design Mach number) 53

Table 3.5 Test cases for the high-turning cascades: SL1, SL2 and SL2P (design and off-

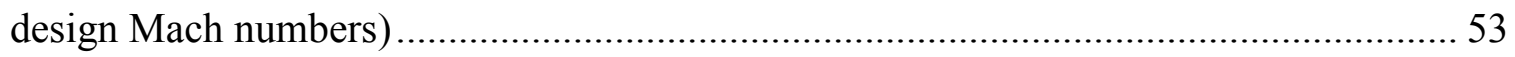

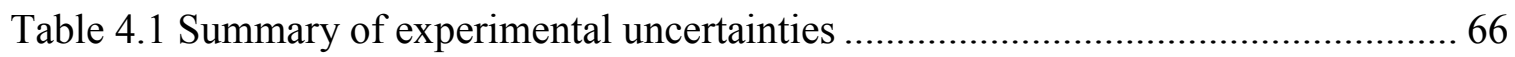

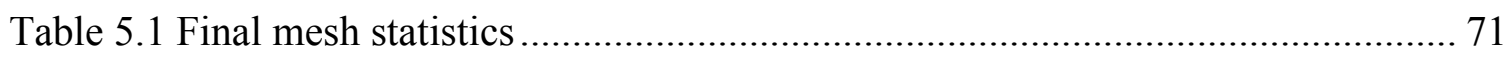

Table 5.2 3D grid convergence study: mass-averaged results (SL2F) .......................... 79

Table 5.3 Inlet turbulence length scale variations (transitional simulations) ................... 80

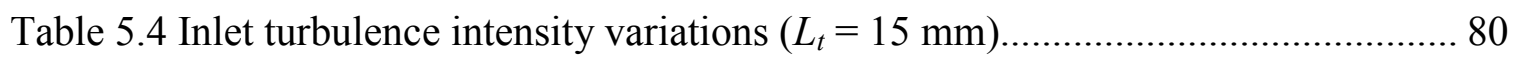

Table 6.1 Summary of cascade parameters at the design point $\left(M_{2, i s}=0.8\right) \ldots \ldots \ldots \ldots \ldots \ldots . . .86$

Table 6.2 Summary of cascade parameters at off-design Mach numbers ...................... 87

Table 6.3 Summary of the experimental and computational investigations .................... 87

Table 6.4 SL2 computational investigations: Mach number variation at constant Reynolds

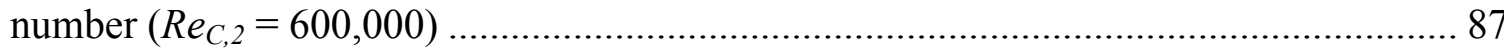

Table 6.5 SL2 computational investigations: Reynolds number variation at constant Mach

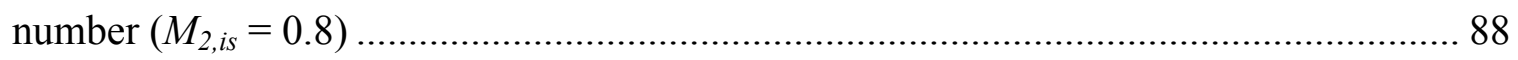

Table 6.6 Mixed-out loss coefficients at tested Mach numbers.................................... 104

Table 6.7 Comparisons with the A-M profile loss correlation $\left(M_{2}=0.8\right) \ldots \ldots \ldots \ldots \ldots \ldots \ldots . . . . . . .108$

Table 6.8 Comparisons with the K-O profile loss correlation $\left(M_{2}=0.8\right) \ldots \ldots \ldots \ldots \ldots \ldots \ldots . . . . . . .110$

Table 7.1 Integrated endwall boundary layer parameters for tested Mach numbers ...... 113 
Table 7.2 Summary of cascade parameters: low-turning airfoils 114

Table 7.3 Mass-Averaged Results for SL3F and SL4F at $1.4 C_{X}$ 125

Table 7.4 Summary of cascade parameters: high-turning airfoils 126

Table 7.5 Overall integrated results at the design Mach number 147

Table 7.6 Overall integrated results at off-design Mach numbers (high-turning cascades)

Table 7.7 Mixed-out total pressure loss coefficients based on the method of Benner et al. (2006a) at the design Mach number.

Table 7.8 Mixed-out total pressure loss coefficients based on the classical lossbreakdown method at the design Mach number ..... 149

Table 8.1 Endwall spanwise displacement due to contouring 153

Table 8.2 Overall integrated results for SL1F and SL1C at the design Mach number... 172 Table 8.3 Overall integrated results for SL1F and SL1C at three different Mach numbers 173

Table 8.4 Overall integrated results for SL2F and SL2C at three different Mach numbers 173

Table 9.1 Overall integrated results for SL2F, SL2C and SL2P at the design Mach number. 188

Table 9.2 Integrated results for SL2F and SL2P at three different Mach numbers $\left(1.4 C_{X}\right)$ 188 


\section{List of Figures}

Figure 1.1 PW6000 gas turbine engine schematic (www.pw.utc.com) ............................. 1

Figure 2.1 Aerodynamic features in a turbine cascade (reproduced from Sjolander, 2006)

Figure 2.2 Turbine blade loading distribution (reproduced from Sjolander, 2006)......... 12

Figure 2.3 Endwall flow structures (reproduced from Wang et al., 1997) ....................... 14

Figure 2.4 Endwall unloading results: front-loaded versus aft-loaded distributions

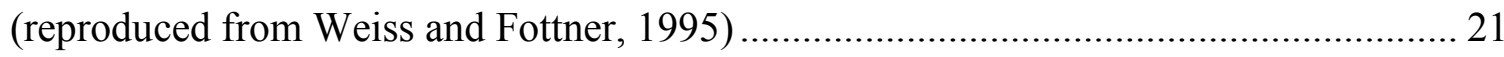

Figure 2.5 Turbine blade loading distribution: midspan loading versus endwall loading

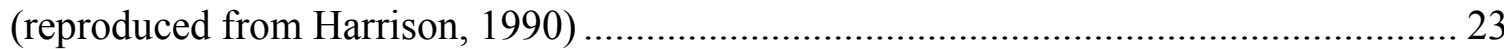

Figure 2.6 Path of the passage vortex with and without the endwall fence (reproduced from Chung et al., 1991) ................................................................................... 26

Figure 2.7 Turbine endwall contouring (left) and airfoil pressure-side modification (right)

Figure 2.8 Application of leading edge bulbs to the T106 airfoil (reproduced from Sauer, 2001) 30

Figure 2.9 Modification of the airfoil pressure-side profile (reproduced from Brear, 2002)

Figure 3.1 High-Speed Wind Tunnel Laboratory at Carleton University ......................... 33

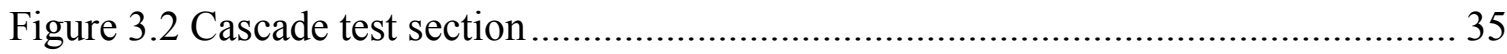

Figure 3.3 Seven-hole probe (top) and Kiel probe (bottom) .......................................... 36

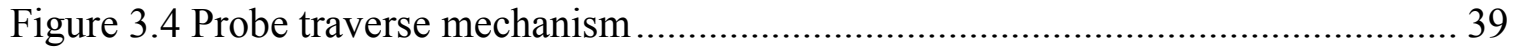

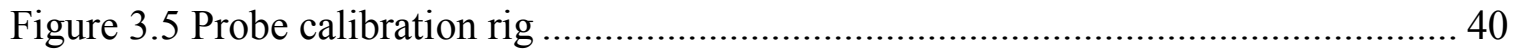

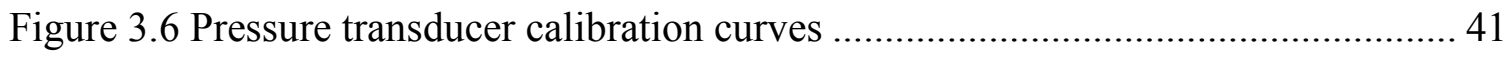

Figure 3.7 Kiel probe and seven-hole probe flow angularity response ........................... 42 
Figure 3.8 Seven-hole probe calibration coefficients (Mach 0.78): $C_{\alpha}$ (top-left), $C_{\beta}$ (top-

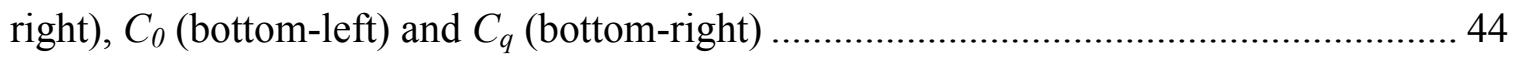

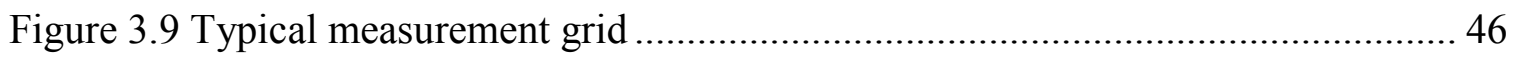

Figure 3.10 Inlet and outlet Mach number distributions based on static tap pressures .... 48

Figure 3.11 Blade surface isentropic Mach number distributions ................................ 48

Figure 3.12 Outlet flow periodicity results at the design Mach number ....................... 49

Figure 3.13 Turbine cascade nomenclature (reproduced from Corriveau, 2005)............ 51

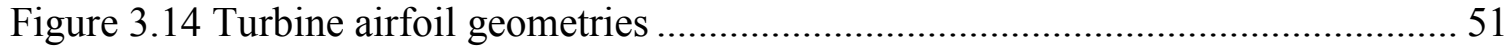

Figure 4.1 Enthalpy-entropy diagram for turbine (reproduced from Denton, 1993)....... 55

Figure 4.2 Suction surface definition for the new loss breakdown scheme (reproduced

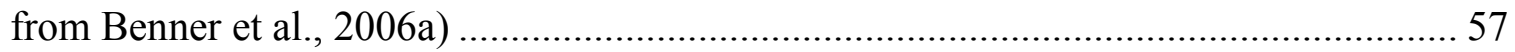

Figure 4.3 Turbine blade loading distribution, and inlet and outlet flow angles

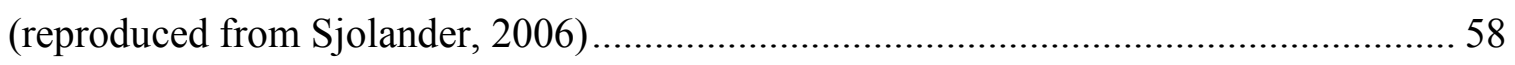

Figure 4.4 Relative pressure uncertainty for the pressure range in the high-speed wind

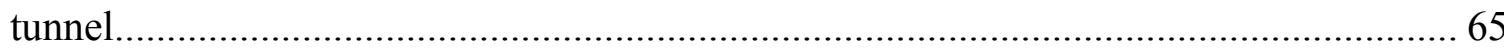

Figure 5.1 Inlet (pre-domain) configuration and computational mesh .......................... 68

Figure 5.2 Computational domain and 2D surface mesh for SL2F .............................. 69

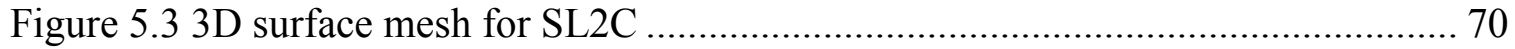

Figure 5.4 CFD results at the outlet of the pre-domain: Mach number ratio, turbulence

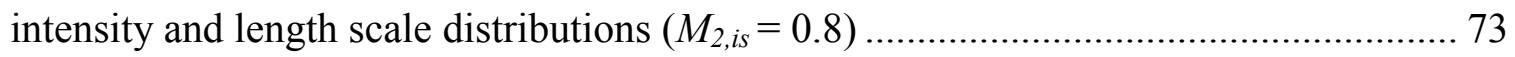

Figure 5.5 Convergence history for one of the 2D simulations for SL2F: RMS residuals

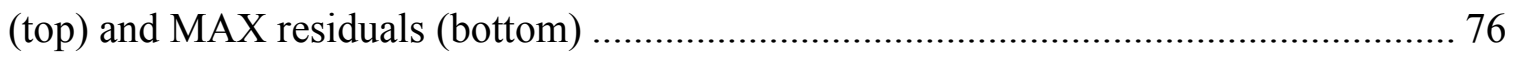

Figure 5.6 Grid convergence study for SL2F (2D simulations): total pressure deficit at $2.0 C_{X}$ (left) and integrated quantities (right) 77

Figure 5.7 Iterative convergence study for SL2F (2D simulation): total pressure deficit (left), loss coefficients and exit flow angles (right). 77

Figure 5.8 3D grid convergence study (SL2F): colour floods of energy loss coefficient 79 Figure 5.9 Effects of inlet eddy viscosity ratio $(E V R)$ on turbulence decay .................... 81 Figure 5.10 Effects of inlet eddy viscosity ratio on skin friction coefficients $\left(C_{f}\right)$ and mixed-out profile loss coefficients for SL2F $\left(M_{2, i s}=0.8\right)$ 81 
Figure 5.11 Effects of inlet turbulence intensity $(T u)$ on skin friction coefficients $\left(C_{f}\right)$ and mixed-out profile loss coefficients for SL2F $\left(M_{2, i s}=0.8\right)$ 83

Figure 6.1 High turning airfoils: blade surface Mach numbers versus axial chord location (left) and normalized surface length (right) at the design Mach number $\left(M_{2, i s}=0.8\right) \ldots . .89$ Figure 6.2 Low turning airfoils: blade surface Mach numbers versus axial chord location (left) and normalized surface length (right) at the design Mach number $\left(M_{2, i s}=0.8\right) \ldots . .90$ Figure 6.3 Pressure-side modified airfoil: blade surface Mach numbers versus axial chord location (left) and normalized surface length (right) at the design Mach number $\left(M_{2, i s}=\right.$ $0.8)$ 91

Figure 6.4 Blade surface Mach numbers at the design Mach number $\left(M_{2, i s}=0.8\right) \ldots \ldots \ldots . .92$ Figure 6.5 Pressure surface acceleration parameters at the design Mach number........... 92 Figure 6.6 Suction surface acceleration parameters at the design Mach number............ 94 Figure 6.7 Blade surface skin friction coefficients from CFD $\left(M_{2, i s}=0.8\right) \ldots \ldots \ldots \ldots \ldots \ldots . . . .94$ Figure 6.8 Midspan surface flow visualization results at the design Mach number........ 95 Figure 6.9 Blade surface Mach number distributions at off-design Mach numbers........ 97 Figure 6.10 Mach number variation in SL2: surface Mach numbers and skin friction

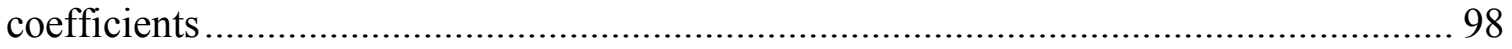

Figure 6.11 Mach number variation in SL2: $C_{P S, Z w}, Z w$ and $\beta_{2}$ distributions ................ 99 Figure 6.12 Reynolds number variation in SL2: $M_{i s}$ and $C_{f}$ distributions ..................... 100 Figure 6.13 Profile flow measurements at the design Mach number at $1.4 C_{X}$ : energy loss

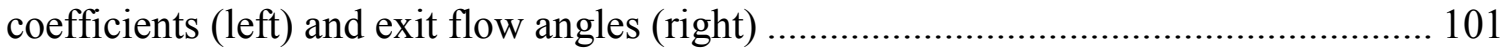

Figure 6.14 CFD predictions at the design Mach number at $1.4 C_{X}$ : energy loss

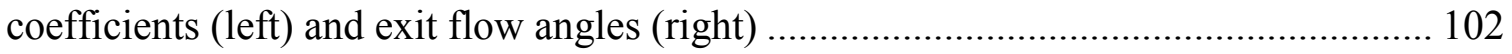
Figure 6.15 Mixed-out energy loss coefficients: experiments (left) and CFD (right).... 103 Figure 6.16 Effects of Mach number variation for SL2 at $2.0 C_{X}: \Delta P_{0} / P_{01}$ (left) and $\zeta$ (right) ...... 105

Figure 6.17 The effects of Mach number variation on the predicted loss coefficients for SL2 $\left(\operatorname{Re}_{C, 2}=600,000\right)$ 106

Figure 6.18 The effects of Reynolds number variation on the predicted loss coefficients for SL2 $\left(M_{2, i s}=0.8\right)$ 106 
Figure 6.19 Ainley and Mathieson (1951) profile loss correlations for nozzles (left) and impulse blades (right) 108

Figure 6.20 Components of the K-O loss correlation at $M_{2}=0.8$ : trailing-edge-thickness effects (left) and subsonic Mach number correction (right) .... 110

Figure 7.1 Inlet endwall boundary layer measurements 113

Figure 7.2 Low-turning airfoils: midspan blade loading distributions $\left(M_{2, i s}=0.78\right) \ldots . .114$

Figure 7.3 Downstream measurement plane and secondary flow features................... 115

Figure 7.4 Low-turning cascades: energy loss coefficient contour plots....................... 116

Figure 7.5 (a) Colour floods of local axial velocity density ratio superimposed over contour lines of energy loss coefficient, and (b) pitch-averaged energy loss coefficients 117

Figure 7.6 (a) Streamwise vorticity coefficients, (b) pitch-averaged enstrophy coefficients and (c) exit flow angles.....

Figure 7.7 (a) Secondary kinetic energy coefficient contour plots, and (b) pitch-averaged results at $1.4 C_{X}$ 122

Figure 7.8 Downstream development of secondary loss distributions for SL3F. 124

Figure 7.9 Pitch-averaged energy loss coefficients for SL3F 125

Figure 7.10 High-turning airfoils: midspan loading distributions at design conditions . 127 Figure 7.11 High-turning airfoils: midspan loading distributions at off-design conditions 127

Figure 7.12 Surface flow visualization results $\left(M_{2, i s}=0.78\right)$ : airfoil suction surfaces ... 128 Figure 7.13 Surface flow visualization results $\left(M_{2, i s}=0.78\right)$ : endwalls. 129

Figure 7.14 (a) Colour floods of local axial velocity density ratio superimposed over contour lines of energy loss coefficient, and (b) pitch-averaged energy loss coefficients $\left(M_{2}=0.78\right)$. 132

Figure 7.15 Streamwise vorticity coefficients $\left(C_{\omega s}\right)$ at three different exit Mach numbers

Figure 7.16 Secondary kinetic energy coefficients at three different exit Mach numbers 136

Figure 7.17 Pitch-averaged results at $1.4 C_{X}$ : (a) energy loss coefficients, (b) secondary kinetic energy coefficients and (c) exit flow angles 138 
Figure 7.18 (a) Secondary kinetic energy coefficients at $2.0 C_{X}$, (b) pitch-averaged $C_{S K E}$ and (c) pitch-averaged energy loss coefficients $\left(M_{2, i s}=0.78\right)$ 140

Figure 7.19 Blade loading distributions at various spanwise locations $\left(M_{2, i s}=0.78\right) \ldots 142$ Figure 7.20 3D CFD results: colour floods of streamwise vorticity coefficient superimposed over contour lines of energy loss coefficient $\left(M_{2, i s}=0.78\right)$ 143

Figure 7.21 Experimental and numerical pitch-averaged results for SL1F at $1.4 C_{X}$ : exit flow angles, $S K E$ coefficients and energy loss coefficients $\left(M_{2, i s}=0.78\right) \ldots \ldots \ldots \ldots \ldots \ldots \ldots \ldots . . . .145$ Figure 7.22 Experimental and numerical pitch-averaged results for SL2F at $1.4 C_{X}$ : exit flow angles, $S K E$ coefficients and energy loss coefficients $\left(M_{2, i s}=0.78\right)$..... 146 Figure 8.1 Example distribution of endwall control points (reproduced from Praisner et al., 2007) 152

Figure 8.2 Optimized contoured endwall geometries 153

Figure 8.3 Endwall height contours $\left(z / C_{X}\right)$... 154

Figure 8.4 Midspan blade loading distributions $\left(M_{2, i s}=0.78\right)$. 155

Figure 8.5 CFD predictions: endwall isentropic Mach number distributions overlaid on velocity vectors at $0.6 \mathrm{~mm}$ above the endwall surface 156

Figure 8.6 Endwall surface flow visualization results $\left(M_{2, i s} \approx 0.8\right)$ : (a) SL1F and (b) SL1C 158

Figure 8.7 Endwall surface flow visualization results $\left(M_{2, i s} \approx 0.8\right)$ : (a) SL2F and (b) SL2C 159

Figure 8.8 (a) Colour floods of streamwise vorticity coefficient overlaid on contour lines of mass-weighted energy loss coefficient and (b) pitch-averaged exit flow angles at $1.4 C_{X}$ 161

Figure 8.9 (a) Secondary kinetic energy coefficients and secondary velocities and (b) pitch-averaged $C_{S K E}$ at $1.4 C_{X}$ 164

Figure 8.10 (a) Secondary kinetic energy coefficients and secondary velocities and (b) pitch-averaged $C_{S K E}$ at $2.0 C_{X}$ 165

Figure 8.11 CFD predictions for SL2F and SL2C: colour floods of streamwise vorticity coefficient overlaid on contour lines of mass-weighted energy loss coefficient $\left(M_{2, i s}=\right.$ $0.78)$ 168 
Figure 8.12 Experimental and numerical pitch-averaged results for SL1F and SL1C at $1.4 C_{X}$ : exit flow angles, SKE coefficients and energy loss coefficients $\left(M_{2, i s}=0.78\right) \ldots 169$ Figure 8.13 Experimental and numerical pitch-averaged results for SL2F and SL2C at $1.4 C_{X}$ : exit flow angles, SKE coefficients and energy loss coefficients $\left(M_{2, i s}=0.78\right) \ldots 169$ Figure 8.14 Pitch-averaged energy loss coefficients: (a) $1.4 C_{X}$ and (b) $2.0 C_{X} \ldots \ldots \ldots \ldots . . .170$ Figure 9.1 Airfoil geometries and midspan loading distributions $\left(M_{2, i s}=0.78\right) \ldots \ldots \ldots \ldots . . .177$ Figure 9.2 CFD predictions: endwall isentropic Mach number distributions overlaid on

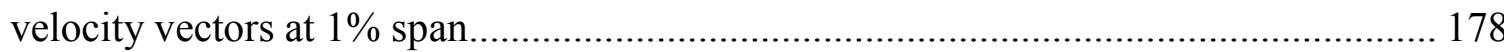

Figure 9.3 Suction surface flow visualization results ............................................ 179

Figure 9.4 Endwall surface flow visualization results: (a) SL2F and (b) SL2P ............ 180 Figure 9.5 (a) Streamwise vorticity coefficients overlaid on contour lines of massweighted energy loss coefficient and (b) pitch-averaged exit flow angles at $1.4 C_{X} \ldots \ldots 181$ Figure 9.6 (a) Secondary kinetic energy coefficients and secondary velocities, (b) pitchaveraged $C_{S K E}$ and (c) pitch-averaged energy loss coefficients at $1.4 C_{X}$ 183 Figure 9.7 (a) Secondary kinetic energy coefficients and secondary velocities, (b) pitchaveraged $C_{S K E}$ and (c) pitch-averaged energy loss coefficients at $2.0 C_{X}$ 184

Figure 9.8 CFD predictions for SL2F and SL2P: colour floods of streamwise vorticity coefficient overlaid on contour lines of mass-weighted energy loss coefficient $\left(M_{2, i s}=\right.$ $0.78)$

Figure 9.9 Experimental and numerical pitch-averaged results for SL2F and SL2P at $1.4 C_{X}$ : exit flow angles, $S K E$ coefficients and energy loss coefficients $(\mathrm{M} 2$, is $=0.78) .187$ Figure 9.10 Mixed-out loss coefficient variation with Mach number from three different axial locations for SL2P 189 


\section{List of Symbols}

$$
\begin{array}{ll}
a & =\text { speed of sound } \\
A & =\text { area } \\
A C & =\text { acceleration parameter (Equation 4.15) } \\
A V R & =\text { axial velocity ratio (Equation 4.11) } \\
A V D R & =\text { axial velocity density ratio (Equation } 4.10) \\
C & =\text { airfoil chord length } \\
C R & =\text { convergence ratio (Equation } 4.9) \\
C_{f} & =\text { skin friction coefficient (Equation } 4.16) \\
C_{P S, Z w} & =\text { static-pressure coefficient (Equation } 6.1) \text { for comparisons with } Z w \\
C_{0} & =\text { seven-hole probe total pressure coefficient } \\
C_{q} & =\text { seven-hole probe dynamic pressure coefficient } \\
C_{S K E} & =\text { secondary kinetic energy coefficient (Equation } 4.21) \\
C_{v} & =\text { heat capacity at constant volume } \\
c_{p} & =\text { heat capacity at constant pressure } \\
C_{X} & =\text { airfoil axial chord length } \\
C_{\alpha} & =\text { seven-hole probe pitch angle coefficient } \\
C_{\beta} & =\text { seven-hole probe yaw angle coefficient } \\
C_{\varepsilon} & =\text { enstrophy coefficient (Equation } 4.19) \\
C_{\omega s} & =\text { streamwise vorticity coefficient (Equation 4.22) } \\
D & =\text { diameter } \\
E V R & =\text { eddy viscosity ratio } \\
h & =\text { span; enthalpy } \\
H & =\text { boundary layer shape factor } \\
L_{t} & =\text { integral length scale of turbulence }
\end{array}
$$




$$
\begin{aligned}
& \dot{m}=\text { mass flow rate } \\
& M \quad=\text { Mach number } \\
& M R \quad=\text { Mach number ratio (Equation 4.9) } \\
& o \quad=\text { throat opening } \\
& P \quad=\text { pressure } \\
& R \quad=\text { gas constant for air } \\
& \text { Re } \quad=\text { Reynolds number } \\
& R V R \quad=\text { row velocity ratio (Equation 4.9) } \\
& s \quad=\text { blade pitch or spacing; entropy } \\
& S \quad=\text { suction-surface coordinate } \\
& \text { SKE = secondary kinetic energy } \\
& \text { SSL = suction-surface length } \\
& t \quad=\text { time; airfoil thickness } \\
& T=\text { temperature } \\
& \text { TET = trailing-edge thickness } \\
& T K E \quad=\text { turbulence kinetic energy } \\
& \mathrm{Tu} \quad=\text { turbulence intensity } \\
& U \quad=\text { velocity } \\
& u, v, w=\text { velocity components in axial, pitchwise and spanwise directions } \\
& v_{s e c}, w_{s e c}=\text { components of secondary velocity vector (Equation } 4.20 \text { ) } \\
& V \quad=\text { velocity; vortex } \\
& W \quad=\text { airfoil leading edge or trailing edge wedge angle } \\
& x=\text { axial coordinate measured from the leading-edge plane } \\
& y, z \quad=\text { local pitchwise and spanwise coordinates } \\
& y+\quad=\text { non-dimensional wall distance } \\
& Y \quad=\text { total pressure loss coefficient (Equation 4.5) } \\
& Z_{T E} \quad=\text { spanwise distance of the } \mathrm{S}_{4} \text { separation line from the endwall at the trailing } \\
& \text { edge (Equation 4.8) } \\
& \text { Zw } \quad=\text { Zweifel coefficient (Equation 4.12) } \\
& \alpha \quad=\text { flow angle measured from the axial direction } \\
& \beta \quad=\text { airfoil metal angle measured from the axial direction }
\end{aligned}
$$




$$
\begin{array}{ll}
\gamma & =\text { stagger angle measured from the axial direction; specific heat ratio } \\
\delta_{99 \%} & =99 \% \text { boundary-layer thickness } \\
\delta * & =\text { boundary-layer displacement thickness } \\
\varepsilon & =\text { enstrophy } \\
\zeta & =\text { energy loss coefficient (Equation } 4.6) \\
\theta & =\text { boundary-layer momentum thickness; uncovered turning } \\
\mu & =\text { dynamic viscosity } \\
\nu & =\text { kinematic viscosity } \\
\rho & =\text { density } \\
\tau & =\text { shear stress } \\
\varphi & =\text { flow misalignment relative to the probe } \\
\omega & =\text { vorticity (Equation 4.18) }
\end{array}
$$

\section{Subscripts}

$$
\begin{array}{ll}
0 & =\text { total } \\
1,2 & =\text { cascade inlet and outlet } \\
C L & =\text { centreline value at the inlet boundary layer traverse plane } \\
\text { is } & =\text { isentropic } \\
L E & =\text { leading edge } \\
\text { max } & =\text { maximum } \\
M S & =\text { midspan } \\
P & =\text { profile } \\
\text { prim } & =\text { primary } \\
\text { ref } & =\text { reference } \\
S & =\text { static; secondary } \\
\text { sec } & =\text { secondary } \\
T E & =\text { trailing edge } \\
\text { tip } & =\text { tip-leakage } \\
w & =\text { wall value }
\end{array}
$$$$
x, y, z, s=\text { components in axial, pitchwise, spanwise and streamwise directions }
$$ 


\section{Superscripts}
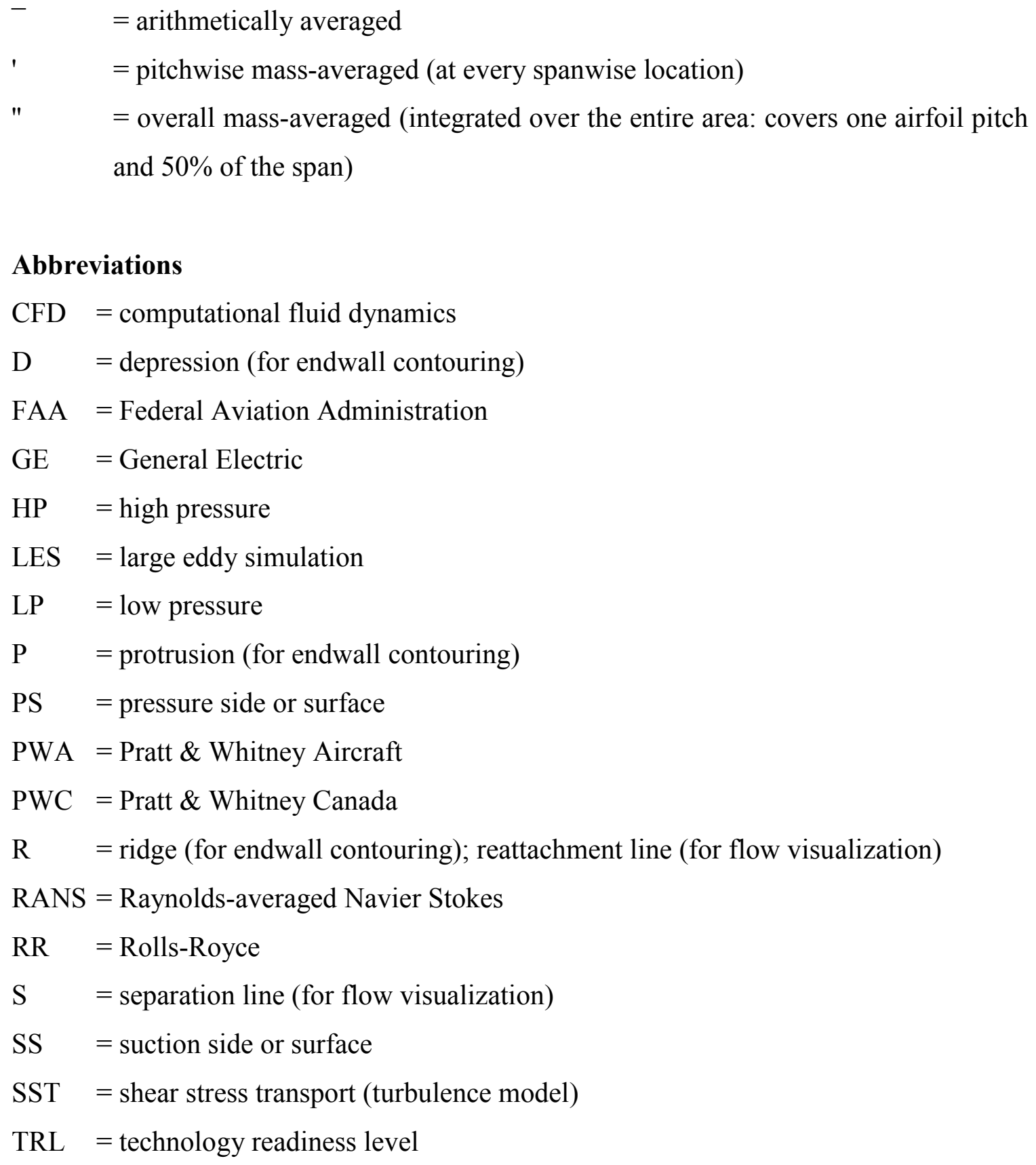


\section{Chapter 1}

\section{Introduction}

\subsection{Motivation}

In order to improve the efficiency and reduce the weight, capital cost and manufacturing costs of an aircraft gas turbine engine, all engine components need to be optimized, including the fan, the compressor, the combustor and the turbine. As presented in Figure 1.1, the turbine section of an aircraft gas turbine engine typically consists of both high-pressure (HP) and low-pressure (LP) turbine stages. The present study is concerned with the aerodynamic design and optimization of the HP turbine stages of aircraft engines, with particular focus on the highly three-dimensional flows near the endwalls of the blade rows.

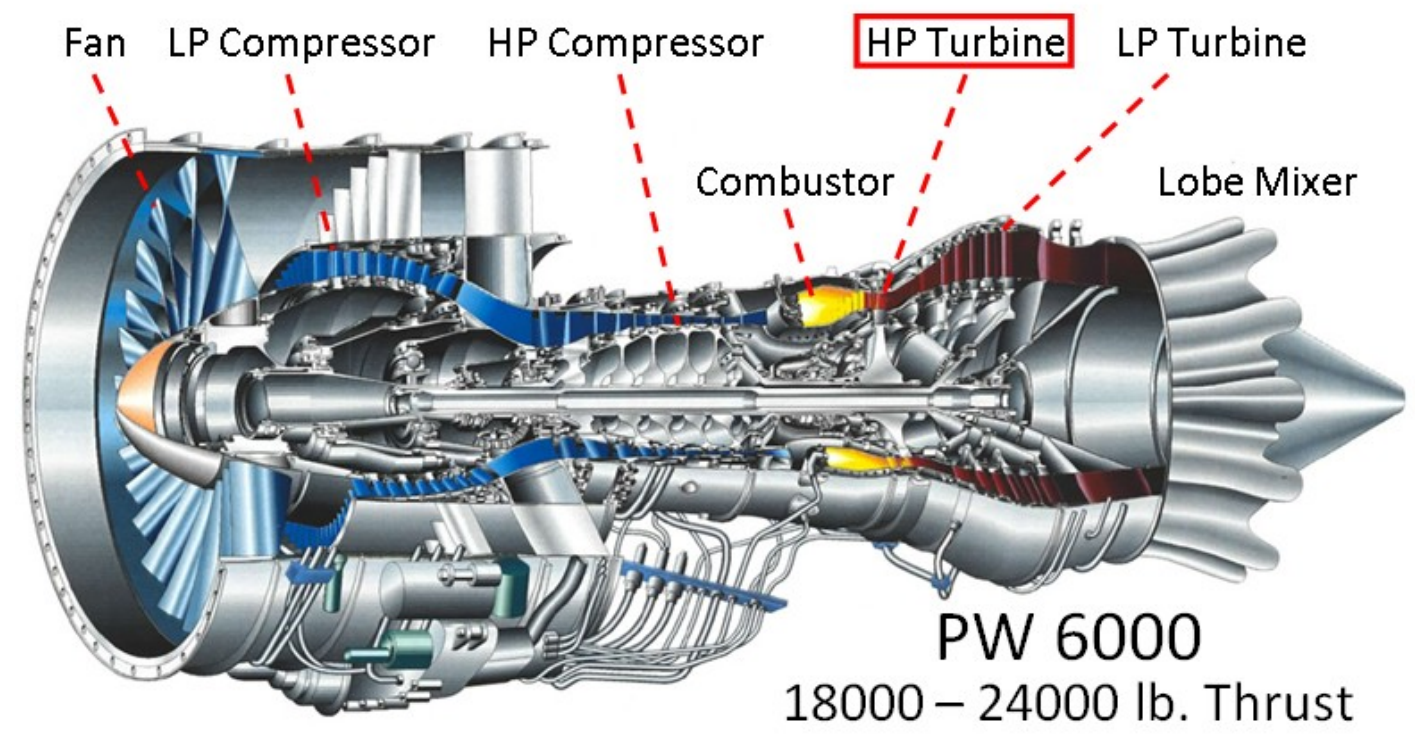

Figure 1.1 PW6000 gas turbine engine schematic (www.pw.utc.com) 
Since the advent of aircraft gas turbine engines, all engine manufacturers, including Pratt \& Whitney Aircraft (PWA), General Electric (GE) and Rolls-Royce (RR) have been conducting research with the aim of reducing engine cost and increasing engine efficiency. Recent aircraft engine research has been focused on reducing the weight and parts count, and thus reducing the cost of the engine.

The current research project was initiated in 2005 under contract from PWA. The technologies that are investigated here will find applications in future generations of PWA engines, including engines for use in the replacements for the Boeing 737 and Airbus A320 families. Operating data for these aircraft are presented in Table 1.1. As shown, the aircraft families combine for about 13000 units delivered through 2012, and an additional 6600 units on firm orders.

Table 1.1 Boeing 737 and Airbus A320 data (www.boeing.com and www.airbus.com)

\begin{tabular}{|c|c|c|}
\hline & Airbus A320 Family & Boeing 737 Family \\
\hline Seating Capacity & $100-220$ & $100-220$ \\
\hline Range $(\mathrm{km})$ & $3100-12000$ & $3500-10000$ \\
\hline Available Engines & CFM56, V2500 , PW6000 & CFM56 \\
\hline Units Delivered (Through 2012) & +5400 & +7400 \\
\hline Firm Orders (Through 2013) & +3600 & +3000 \\
\hline
\end{tabular}

The PW6000 engine, presented in Figure 1.1, is one of the engines available on the Airbus A319 aircraft. The International Aero Engines' V2500 engine, developed in part by PWA, has a small share of the A320 family as well. The PW1000G, which is a high by-pass geared turbofan engine, is also aimed at the single-aisle aircraft market. In order to attract more interest from Boeing and Airbus in this market, PWA needs to offer more efficient, light-weight engines, which can compete with GE and RR engines. To this end, the turbine aerodynamics group at PWA has been conducting research to optimize the design of both HP and LP turbine stages.

An important objective of the turbine aerodynamics group is to minimize the pressure losses through the turbine passages. Since modern turbine design is strongly influenced by the cost and the weight of the engine, reducing the number of the turbine blades has become important in the design. In HP turbines, reducing the number of blades introduces other positive benefits such as cooling flow reductions, as well as turbine disk 
and casing weight reductions. In order to extract the same work output from the turbine stages, decreasing the number of turbine blades would result in higher blade loadings. Increasing the blade loading, however, could potentially result in higher pressure losses and thus lower engine efficiencies.

In order to achieve the various and sometimes conflicting objectives, computational fluid dynamics (CFD) has been employed increasingly in recent years. By the mid 1990s, most engine manufacturers relied heavily on their CFD predictions. The CFD predictions, however, showed some shortcomings due to imperfect modelling of the complicated three-dimensional flows, particularly near the endwalls of the turbines. Furthermore, while correlations based on experimental data from the 1950s and 1960s have been the basis for the preliminary design of most modern turbine stages, due to advances in turbine design, a requirement has arisen to conduct further experimental research both to validate the CFD predictions and to obtain improved design correlations. Once the numerical results have been validated experimentally, CFD can be used with greater confidence in order to expand the turbine design envelope.

\subsection{Research Objectives}

In addition to supporting the broader goals identified in the last paragraph, the present research project is also aimed at improving the understanding of the three dimensional flows near the endwalls in transonic turbines. In order to achieve this objective, both experimental and computational research was conducted in collaboration with PWA. The results from several different cascade geometries at both design and off-design Mach numbers are presented, particularly to examine the influence of airfoil loading on total pressure losses and loss reduction technologies.

The research has fundamental objectives in that it is aimed at obtaining new physical insights into the highly three-dimensional flows inside turbine cascades. This is particularly relevant since experimental data from transonic turbine cascades are scarce in the open literature. The research is also practical since the new technologies for reducing the secondary losses, which are investigated, may be implemented in the next generation of aircraft gas turbine engines. In this regard, the experimental results may be used to improve the CFD modelling and to devise loss correlations. 
The first part of the research project consists of experimental investigations of the three-dimensional flows in a family of five transonic linear turbine cascades. These cascades have been designed and manufactured with flat endwalls on both sides. The main objective of this segment of the research project is to study the three-dimensional flow features near the endwalls, as well as to investigate the aerodynamic performance of some modern turbine airfoil designs. The three-dimensional flow features in turbine blade passages are responsible for generating total pressures losses, commonly referred to as secondary losses. Secondary losses are particularly important in HP turbine stages due to the smaller airfoil aspect ratios.

The second phase of the research project is concerned with the aerodynamic performance of cascades with contoured endwalls. These cascades use the same turbine airfoil geometries as the flat endwall cascades; however, one endwall has been contoured with the aim of reducing the secondary losses. The endwall contouring design was carried out by PWA using CFD-based optimization software. The main goal here is to experimentally investigate the physical effects of non-axisymmetric endwall contouring on the secondary flows and the consequent secondary losses.

The third phase of the research project is focused on the performance of a so-called "pressure-side" modified cascade. The pressure-side modification is also aimed at controlling the secondary flow structures and reducing the secondary losses without deteriorating the two dimensional flow characteristics.

Finally, complementary CFD investigations were carried out for both 2D and 3D aspects of the flows. These assisted in the interpretation of the experimental results since the simulations can provide approximate results in areas of the flow that are not accessible with the instrumentation. In regions of the flow where both experimental and computational data were available, it was also possible to assess the ability of CFD to predict the more subtle features of these flows. In addition, CFD results were used to verify the correct functioning of some of the data reduction software.

\subsection{Overview of Thesis}

The review of the available literature, pertinent to the current project, is presented in Chapter 2. Detailed comparisons between the results of the current work and the 
previous findings, however, are postponed until later chapters as appropriate. The experimental investigations were conducted at the Pratt and Whitney Canada High-Speed Wind Tunnel Laboratory at Carleton University. The experimental data includes pressure probe measurements and surface flow visualization results. The experimental apparatus and measurements procedures are described in Chapter 3.

The data reduction procedures and experimental uncertainty analysis are presented in Chapter 4; these include the definitions of the loss coefficients, the secondary loss parameters and the data averaging techniques. The computational domain, the CFD simulation settings, the boundary conditions and the numerical convergence analysis are presented in Chapter 5. The computational investigations were conducted using the commercial CFD code ANSYS CFX (versions 11 and 12). The effects of varying the inlet turbulence parameters on the CFD predictions are also investigated and presented in Chapter 5 since the turbulence model is one of the major contributors to the numerical uncertainties.

The results of the experimental and numerical investigations are presented in Chapters 6 through 9. The midspan blade loading distributions and profile losses at both design and off-design Mach numbers are examined in Chapter 6. The experimental results are also compared both with the numerical predictions and empirical profile loss correlations. The secondary loss results from the cascades with flat endwalls are discussed in Chapter 7. The secondary flow fields are characterized using both probe measurements and surface flow visualization results. Furthermore, the probe data from two different measurement planes are used to investigate the flow mixing processes downstream of the cascades. The results from the contoured endwall cascades are discussed in Chapter 8. The benefits of endwall contouring are assessed in several ways: in terms of mass-averaged and mixed-out total pressure losses, secondary kinetic energy, flow underturning and overturning, and surface streamline patterns. Endwall contouring is determined to be effective for the more highly loaded cascade, whereas the moderately loaded cascade only shows modest benefit. The airfoil pressure-side modification to reduce the secondary losses is described in Chapter 9. The pressure-side modification significantly reduces the strength of the vortical structures and is determined to be particularly successful in reducing the secondary losses. 
Various areas of improvement have been identified during the course of the experimental investigations; these include improvements to the data collection and data reduction procedures. In addition, it is recognized that the present work represents an idealized approach to the problem. The conclusions from the current work and the recommendations for future work are presented in Chapter 10. The results of this study may be particularly useful for devising secondary loss correlations for endwall contouring and airfoil optimization in the future. 


\section{Chapter 2}

\section{Literature Review}

\subsection{Introduction}

The aerodynamic features of the three-dimensional flows inside turbine blade passages and their relationship to losses are presented in this chapter. To present the relevant background information regarding the research project, the choice of the test procedures also needs to be clarified. As a result, the importance of high-speed linear turbine cascade testing will be discussed to identify some of the strengths and limitations of this experimental approach. The current research project is both fundamental and practical, and thus, the connection of the research to engine technology readiness levels will be described.

The available literature on secondary losses in low-speed and high-speed linear turbine cascades will be presented next. The goal here is to identify some of the geometric and aerodynamic parameters, which may affect secondary flows that are the focus of the present research. These effects include inlet boundary layer thickness, freestream turbulence intensity, blade loading distribution and total flow turning. The implementation of endwall contouring and airfoil 2D profile modification to reduce the secondary losses will also be described.

\subsection{Flow Field and Losses in Turbine Blade Passages}

Figure 2.1 shows schematically the main flow features present in a typical turbine blade passage; cooling flows are omitted here for simplicity. Away from the endwalls, tipwall and hubwall in Figure 2.1, the flow and the boundary layers on the blade surfaces are largely two-dimensional. This is usually referred to as the profile flow (near midspan 
of the cascade). The pressure losses associated with the profile flow are referred to as profile losses and trailing-edge losses:

- Profile losses: The losses generated in the blade-surface boundary layers.

- Trailing-edge losses: Additional losses generated by the shed vortices downstream of the trailing edge through mixing. Trailing edge losses are sometimes considered to be part of the profile losses.

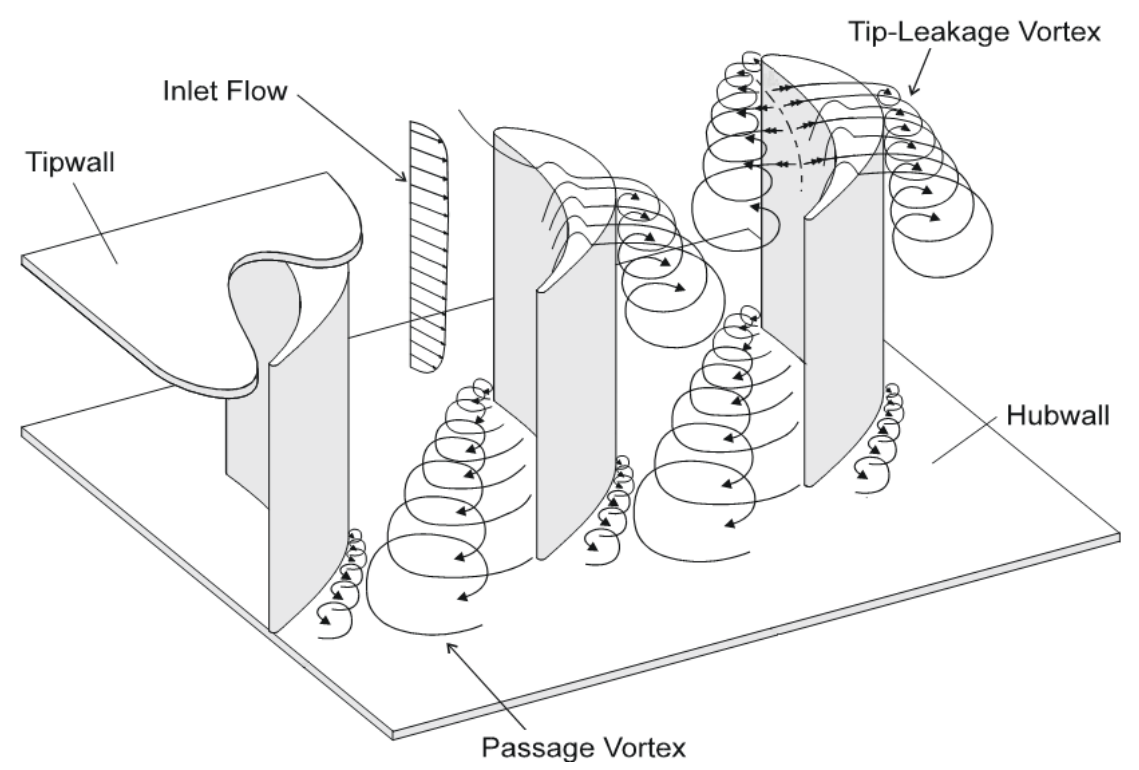

Figure 2.1 Aerodynamic features in a turbine cascade (reproduced from Sjolander, 2006)

Near the endwalls, the flow is much more three dimensional. Considering first the hubwall in Figure 2.1, where there is no clearance between the blade and the endwall, flow three-dimensionality develops as a result of the interaction between the blade pressure distribution and the endwall boundary layer. Since the endwall boundary layer fluid has lower momentum than the mainstream fluid, once it enters the blade passage it is turned more strongly by the blade-to-blade (cross-passage) pressure difference than the mainstream, profile flow. As a result, the endwall flow is driven towards the convex (suction) side of the blade passage. This is known as secondary flow. The secondary flow is blocked by the blade suction surface, and it is thus induced to roll up into a vortex known as the secondary or passage vortex. The pressure losses associated with secondary flows are referred to as secondary losses:

- Secondary losses: The losses generated in the endwall boundary layers, and also through mixing as the vortical structures are convected downstream. 
Next, considering the tipwall in Figure 2.1, flow through the clearance between the blade and the endwall (tip gap) contributes an additional three-dimensional flow feature. As the tip-leakage flow emerges from the suction side of the gap, driven by the pressure difference across the airfoil, it is blocked by the secondary flow and itself rolls up into a vortex known as the tip-leakage vortex. The pressure losses associated with tip-leakage flows are referred to as tip-leakage losses:

- Tip-leakage losses: The main contribution to the tip-leakage losses arises from the mixing out of the tip-leakage vortex with downstream distance.

In the present work, the tip-leakage flow and the associated losses are not of interest. Therefore, the blades are sealed at both ends and there is no tip-clearance.

In addition to the loss sources mentioned, the presence of shock waves can lead to further losses. These losses include the direct loss of total pressure across the shock waves, and additional losses as a result of boundary layer separation induced by shockwave/boundary-layer interaction. It is generally believed that the presence of shock waves has a smaller effect on the endwall boundary layers than on the blade-surface boundary layers, particularly with respect to the overall work extraction. To avoid the additional complexity of secondary flow distortion by shock waves, the outlet Mach numbers are kept low enough so that only weak shock waves will be generated at worst.

The High-Speed Wind Tunnel Laboratory at Carleton University has been used extensively in the past for midspan aerodynamic measurements in transonic linear turbine cascades (Islam, 1999; Jeffries, 2000; Jouini, 2000; Corriveau, 2005). The main focus of the past research projects has been on profile loss measurements. The present study, on the other hand, is focused on secondary flows and secondary losses.

\subsection{Role of Linear Turbine Cascade Research}

The current research is concerned with the highly three-dimensional flows near the endwalls in transonic linear turbine cascades. A linear turbine cascade is a planar row of a finite number of blades that is used to approximate the flow in the annular, effectively infinite row of blades, in an actual machine. The project uses linear turbine cascades that were designed and manufactured by PWA, as described in Chapter 3. 
The linear turbine cascade is an idealized case, which simulates only some of the many flow features present within the turbine stage of an aircraft engine. Some of the flow features, which are present within the turbine stage but are not simulated, include:

- Elevated temperature levels

- Flow unsteadiness due to wakes from upstream blade rows

- Radial pressure gradients

- $\quad$ Blade tip leakage flows

- Blade surface and endwall film cooling flows

- Turbine-disk cooling and leakage flows (purge flows)

- Upstream and downstream platform overlaps (endwall steps)

- Skewed inlet endwall boundary layers due to platform rotation

Some of the flow features simulated in the linear turbine cascade facility include cross-passage and streamwise pressure gradients, free-stream turbulence levels, and flow compressibility. Here, the main objective is to investigate the effects of blade loading distribution, flow turning and endwall contouring on secondary losses in the compressible flow regime.

From an industrial perspective, the limitations of linear turbine cascade testing might raise the question as to what is the value of collecting such experimental data. To answer this question, it is useful to outline the six technology readiness levels (TRL) employed by PWA. The technology readiness levels, presented in Table 2.1, are used to assess the maturity of a new technology from the conceptual phase (level zero: TRLP-0) to possible engine implementation (level six: TRLP-9).

Table 2.1 Technology Readiness Levels used by PWA (TRLP)

\begin{tabular}{|c|c|c|}
\hline TRLP & Description & Example (present study) \\
\hline 0 & Technology concept & Endwall contouring \\
\hline 1 & Feasibility and analytical studies & Euler (inviscid) calculations \\
\hline 2 & Numerical investigations & CFD analysis (3D RANS solver) \\
\hline 3 & $1^{\text {st }}$ stage of experimental investigations & Low-speed cascade testing \\
\hline 4 & $2^{\text {nd }}$ stage of experimental investigations & High-speed cascade testing \\
\hline 5 & $3^{\text {rd }}$ stage of experimental investigations & High-speed rotating test rig \\
\hline 6 & Full scale tests & Engine ground testing \\
\hline 7 & Validation of concept in flight & Flight testing \\
\hline $8-9$ & FAA certification and market introduction & $\mathrm{n} / \mathrm{a}$ \\
\hline
\end{tabular}


As shown Table 2.1, the preliminary design and optimization studies are carried out using CFD analysis (TRL-2) by PWA. However, due to uncertainties associated with turbulence and transition modelling in CFD, the numerical results need to be validated experimentally prior to any further design optimizations.

The effects of endwall contouring on secondary losses have been previously investigated in the low-speed linear cascade facility (TRLP-3) at Carleton University by Knezevici (2011). The research project outlined in this dissertation represents TRLP-4: high-speed linear turbine cascade research. Future technology readiness levels include high-speed rotating rig and engine testing, which will be conducted by PWA.

It should be emphasized that the design is altered using CFD after each stage of experimental testing. In other words, the design is modified several times before it is considered for the engine. Therefore, if a particular design succeeds in the high-speed linear turbine cascade facility (with the highlighted limitations), the same may not be concluded for the engine. This is not the purpose of the current research project. The main goal of the current research is to study secondary flows in the compressible flow regime using linear turbine cascades.

\subsection{Characterization of Blade Loading}

One of the goals of this project is to study the effects of blade loading on the endwall flows and the endwall pressure losses. The blade loading for turbine blades is often specified in terms of a non-dimensional tangential force parameter, known as the Zweifel coefficient, based on the actual and reference loading distributions shown in Figure 2.2.

The reference blade loading distribution in Figure 2.2 is defined as the maximum tangential force that can be obtained for the given inlet and outlet conditions, while avoiding adverse pressure gradients on both sides of the airfoil. This physically unrealizable distribution corresponds to the entire pressure side (PS) at the stagnation pressure $\left(P_{01}\right)$, and the entire suction side $(\mathrm{SS})$ at the minimum static pressure, which is the outlet static pressure $\left(P_{S 2}\right)$. An example of the actual loading distribution is also presented in Figure 2.2. The actual loading distribution exhibits adverse pressure gradients near the leading edge on the pressure side and past the suction peak on the suction side of the airfoil. Based on experimental data from the 1930s and 1940s, 
Zweifel (1945) concluded that $Z w$ should be limited to values below 0.8 in order to minimize the profile losses. In his experiments, higher Zweifel coefficients resulted in larger adverse pressure gradients on the suction surface of the blade, leading to boundary layer separation and higher profile losses.

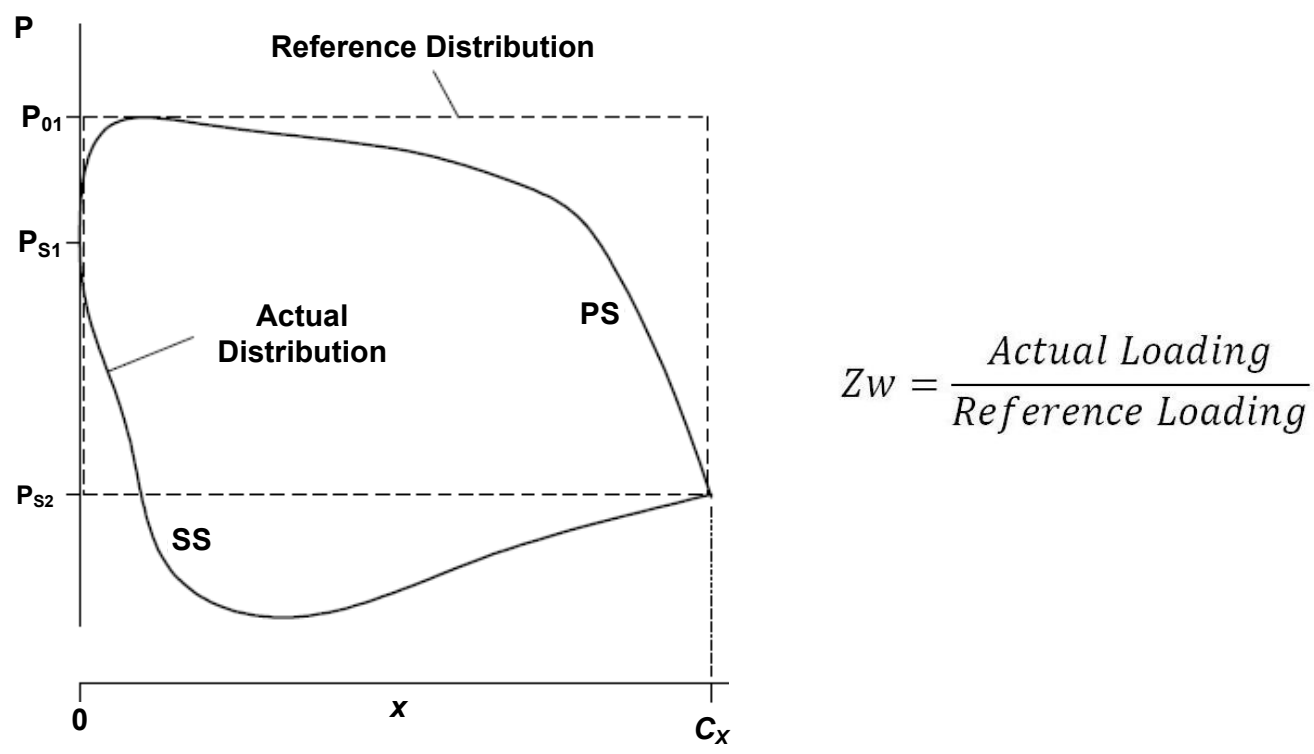

Figure 2.2 Turbine blade loading distribution (reproduced from Sjolander, 2006)

There have been substantial advancements in turbine airfoil design in the last 60 years. Successful low-speed research airfoils (i.e. without boundary layer separation on the suction surface) have been designed with Zweifel coefficients as high as 1.8 (Praisner and Grover, 2008). However, a Zweifel coefficient limit of approximately 0.8 is still widely accepted for HP turbine blades, due in part to a lack of experimental data from high-speed cascade facilities. Within the HP turbine stage, larger Zweifel coefficients, which are traditionally associated with higher profile and secondary losses in subsonic flows, may also lead to higher flow acceleration, accompanied with undesirable strong shock waves. Zweifel coefficients selected for the turbine cascades in the current research are 0.8 and 1.0, which would be regarded as high values of loading for HP turbine airfoils. The goal here is to investigate the effects of the Zweifel coefficient on secondary losses. 


\subsection{Profile and Secondary Losses in Linear Turbine Cascades}

\subsubsection{Profile Losses}

As described in section 2.2, profile losses are defined as the total pressure losses generated within the boundary layers in the $2 \mathrm{D}$ flow region near the midspan of the cascade. Trailing edge losses and losses incurred by the wake mixing process downstream of the blade passage are also included in the profile losses.

Profile losses are strongly influenced by the state of the boundary layer on the suction surface of the blade. The state of the boundary layer is highly dependent on the Reynolds number and the local pressure gradients. At low Reynolds numbers, such as those experienced by LP turbines during cruise, there tends to be a substantial length of laminar boundary layer starting at the leading edge of the blade. This laminar boundary layer is susceptible to separation in the presence of an adverse pressure gradient on the suction surface of the airfoil. Suction-surface boundary layer separation may be followed by:

- boundary layer reattachment, resulting in a separation bubble.

- blade stall, resulting in substantially higher pressure losses and instabilities.

In high-speed wind tunnel facilities, an additional adverse pressure gradient may be introduced by the trailing edge shock wave impinging on the suction surface of the adjacent airfoil. In the current research, the outlet Mach number is selected such to avoid the formation of strong shock waves within the blade passages. Furthermore, due to large Reynolds numbers $\left(\operatorname{Re}_{C, 2} \approx 500,000\right)$ there has not been any evidence of large laminar boundary layer separation. Since the current research is mainly concerned with endwall flows, secondary flows and secondary losses are described next.

\subsubsection{Secondary Flows and Secondary Losses}

The secondary loss, associated with the three-dimensional flow structures near the endwalls, is defined as the difference between the total row loss and the profile loss. This is not a precise description since the profile losses vary across the blade span due to the strong interaction between the vortical structures and the blade-surface boundary layers. However, this description is accepted in the industry since it provides means for defining performance correlations based on specific pressure loss categories (e.g. Kacker and 
Okapuu, 1982). In order to discuss the factors affecting secondary losses in turbine cascades, it is important to first describe the main features of the three-dimensional turbine flow field.

The general interpretation of the flow field inside the turbine blade passage is presented in Figure 2.3. The vortical structures shown in Figure 2.3 were documented by Wang et al. (1997) at a Reynolds number of 27,000 based on the exit velocity and blade chord length. As the inlet flow near the endwall, encounters an adverse streamwise pressure gradient ahead of the leading edge of the turbine blade, the inlet boundary layer rolls up at the saddle point of separation to form a horseshoe vortex. The horseshoe vortex consists of a pressure side leg and a suction side leg. These vortices grow in size and strength inside the passage under the influence of pitchwise (cross-passage), spanwise (radial), and streamwise pressure gradients. Secondary losses are generated as a result of high shear stresses caused by the interaction between the vortical structures and the low-momentum boundary layer fluid within the blade passages.

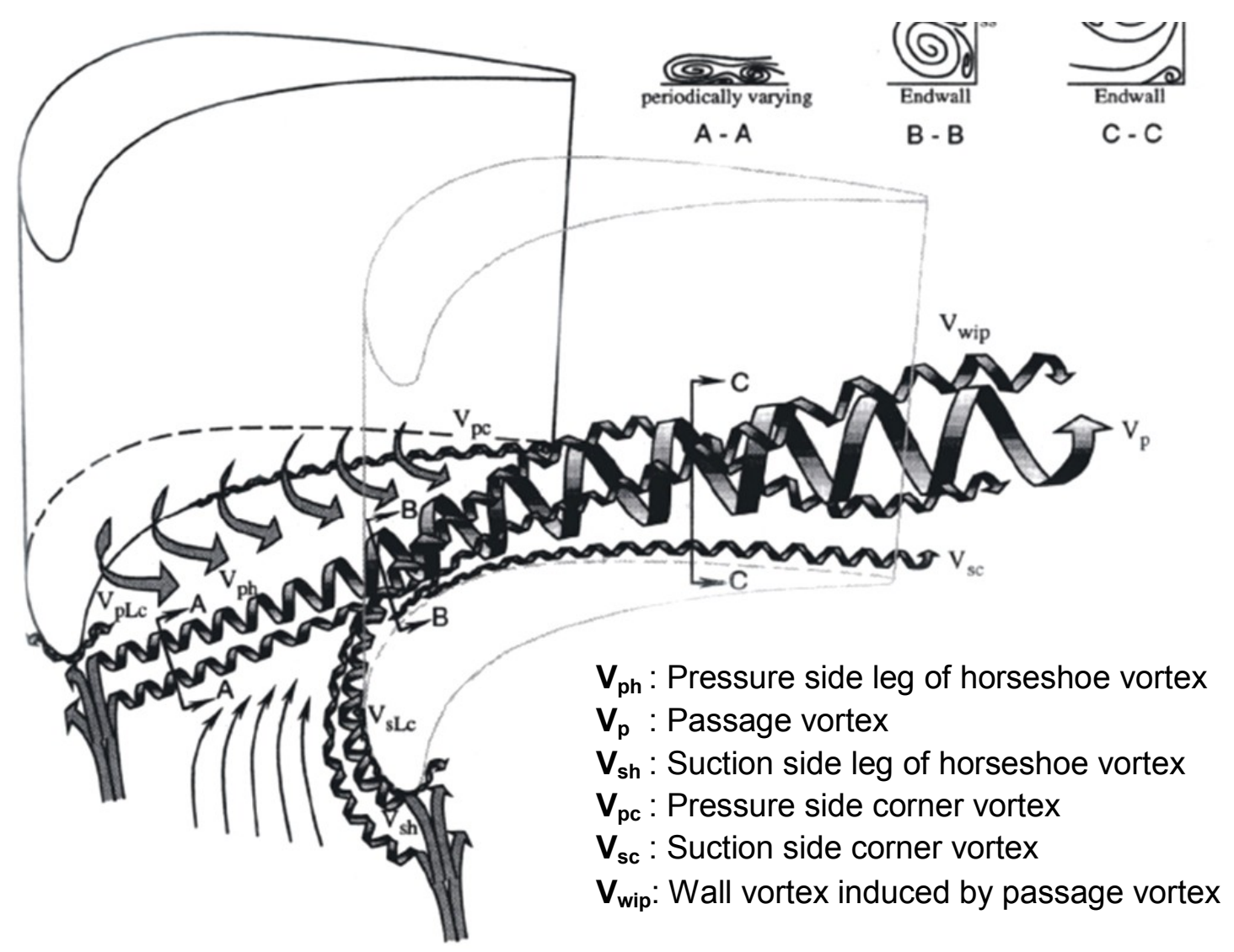

Figure 2.3 Endwall flow structures (reproduced from Wang et al., 1997) 
Looking downstream in Figure 2.3, the pressure side leg of the horseshoe vortex $\left(\mathrm{V}_{\mathrm{ph}}\right)$ has positive vorticity (counter clockwise). Under the action of the cross-passage pressure gradient, the pressure side leg of the horseshoe vortex migrates towards the suction surface of the adjacent blade. In this process, the pressure side leg of the horseshoe vortex entrains low momentum fluid from the endwall boundary layer to form the passage vortex $\left(\mathrm{V}_{\mathrm{p}}\right)$. The growth of the passage vortex is amplified by an adverse streamwise pressure gradient, following the suction peak inside the passage. The passage vortex ultimately reaches the suction surface of the adjacent blade and exhibits strong interaction with the two-dimensional blade-surface boundary layer, as documented by Sieverding (1985). The development of the passage vortex is strongly influenced by the Zweifel coefficient (Dossena et al., 2004), blade loading distribution (Zoric, 2006), leading edge geometry (Benner, 2003) and total flow turning (Yamamoto, 1987a; Yamamoto, 1987b). The interactions between the passage vortex and the other vortical structures also affect the secondary losses. As a result, the geometric features implemented to reduce the secondary losses, are often aimed at reducing the size and strength of the passage vortex.

Looking downstream in Figure 2.3, the suction side leg of the horseshoe vortex $\left(\mathrm{V}_{\mathrm{sh}}\right)$ has negative vorticity (clockwise). The suction side leg of the horseshoe vortex $\left(\mathrm{V}_{\mathrm{sh}}\right)$ may remain close to the suction surface of the blade (Langston, 2001), or otherwise wrap around the passage vortex as shown in Figure 2.3 (Wang et al., 1997). Furthermore, the suction side leg of the horseshoe vortex may remain close to the suction side corner or grow towards the midspan of the cascade depending on the strength and the location of the passage vortex. The interaction between the suction side leg of the horseshoe vortex and the passage vortex is strongly influenced by the cascade aerodynamic parameters.

Other vortical structures shown in Figure 2.3 include pressure side corner vortex $\left(\mathrm{V}_{\mathrm{pc}}\right)$, suction side corner vortex $\left(\mathrm{V}_{\mathrm{sc}}\right)$, and wall vortex induced by the passage vortex $\left(\mathrm{V}_{\text {wip }}\right)$, all of which initiate inside the passage and contribute to the secondary losses.

Secondary flows, and thus secondary losses, are affected by the size, location and strength of the vortical structures. Flow angles at the outlet of the cascade are also affected by the vortical structures. The aerodynamic performance of turbine blades typically declines at off-design inlet incidence as shown by Benner (2003) and Zoric 
(2006). As a result, the spanwise distribution of the outlet flow angles affects the aerodynamic performance of the subsequent blade rows. The following terminology is used to quantitatively compare the spanwise flow angle distributions for different test cases:

- The primary flow direction is identified as the direction of the mass-averaged streamwise component of velocity at midspan of the cascade. The component of flow velocity in the primary flow direction is referred to as the primary velocity. Flow velocity components in the plane normal to the primary flow direction are referred to as secondary velocities.

- Pitchwise-averaged flow angles at other spanwise locations are referred to as flow underturning if they are smaller than the midspan flow angle, and flow overturning if they are larger than the midspan flow angle. The amount of flow underturning and overturning is a function of the size and strength of the passage vortex, and thus linked to secondary losses.

One of the goals of the present study is to investigate the relation between exit flow angle distributions and secondary losses. The flow angle distributions and pressure loss measurements are presented and discussed in Chapters 7 through 9.

\subsection{Linear Turbine Cascade Research on Secondary Losses}

\subsubsection{Introduction}

Secondary losses typically account for about $30 \%$ of total pressure losses in a blade row. Secondary losses produced by low aspect ratio blades, such as HP turbine blades, on the other hand, could contribute up to $50 \%$ of total losses in a blade row (Langston, 2001). As a result, the study of secondary losses has received substantial attention since the 1950s.

Sieverding (1985) provided an excellent review of secondary flow experiments prior to 1985. In his review, he described the vortical structures, the separation and reattachment lines, and the spanwise loss distribution, supplemented with probe measurements and flow visualization results. More recently, secondary flow reviews have been provided by Gregory-Smith (1997) and Langston (2001). 
In the past, secondary loss measurements have been obtained mainly using low-speed linear turbine cascades. Low-speed facilities allow for detailed flow measurements at a reasonable cost. Furthermore, the complications associated with the high aerodynamic forces on measurement probes in high-speed facilities, may be avoided in low-speed facilities. However, the conclusions from low-speed results may not be directly applicable to high-speed flows since the effects of flow compressibility are not simulated.

The current research project is concerned with the effects of flow compressibility on the secondary flow structures. Experimental data on these flows are scarce in the open literature. Therefore, the pertinent literature from both low-speed and high-speed research facilities are reviewed in the next sections. The following parameters are often used to present and compare the secondary flow results from different test cases (see Chapter 4 for derivations and formulas):

- Vorticity is defined as twice the angular velocity of the fluid particles rotating about their own axes. Streamwise vorticity contour plots $\left(\omega_{S}\right)$ are used to determine the location, size and peak vorticity of the vortical structures in the primary flow direction.

- Secondary kinetic energy $(S K E)$ represents the kinetic energy of the flow within the three-dimensional flow region associated with the secondary velocities generated by the vortical structures. In low-speed cascades, $S K E$ production and dissipation has been found to correlate with the rise in total pressure losses (Moore and Adhye, 1985). However, the link between SKE and total pressure losses is not fully understood for transonic flows (Perdichizzi, 1990).

- Turbulence kinetic energy (TKE) represents the mean kinetic energy of the turbulent eddies, often normalized with respect to inlet free-stream velocity. Gregory-smith et al. (1988b) noted that turbulence production is the first step in losing energy since TKE production is followed by dissipation, and energy is lost in this process. The contribution of TKE production to total pressure losses requires further analysis in both low-speed and high-speed cascades.

- The total pressure loss coefficient $(Y)$ is used to quantitatively compare the massaveraged total pressure deficit for different test cases with different outlet dynamic pressures. 
- The energy loss coefficient $(\zeta)$ is also used to quantitatively compare total pressure losses for different test cases. The energy loss coefficient is typically used for compressible flow analysis since it is not highly sensitive to small Mach number variations, associated with blowing pressure fluctuations.

The aerodynamic and geometric parameters presented in the following sections include inlet boundary layer thickness, free-stream turbulence intensity and length scale, blade loading distribution, flow turning and flow compressibility.

\subsubsection{Inlet Boundary Layer Thickness and Free-Stream Turbulence}

For the present study, the inlet endwall boundary layer thickness and the free-stream turbulence levels are not varied. The inlet parameters used for the present study are:

- Turbulent inlet endwall boundary layer $(H \approx 1.4)$

- Inlet endwall boundary layer thickness $\left(\delta_{99 \%}\right)=20 \%$ to $30 \%$ of blade span

- Free-stream turbulence intensity $=4 \%$ (Corriveau, 2005)

- Free-stream turbulence integral length scale $=15 \mathrm{~mm}$ ( $60 \%$ of blade axial chord)

Therefore, the purpose of this section is to examine the importance of the inlet boundary conditions on secondary flows; this is to ensure that the values in the present study are within acceptable ranges, and will not significantly alter the flow fields.

Gregory-Smith et al. (1988a) investigated the effects of inlet endwall boundary layer thickness on secondary flow structures inside a turbine rotor blade passage. Three values of inlet boundary layer thickness were investigated: $18 \%, 22 \%$ and $33 \%$ of blade span. Increasing the inlet boundary layer thickness resulted in less intense, but wider vortical structures within the passage and near the outlet of the cascade. This was attributed to the lower peak vorticity of the thicker inlet boundary layer convecting through the passage, and resulting in lower peak vorticities within and downstream of the cascade. The location of the vortical structures and the net pressure losses were not affected by the boundary layer thickness.

Chen and Dixon (1986) also investigated the effects of inlet boundary layer thickness on secondary losses. They used seven measurement planes (from $15 \%$ to $240 \%$ chord length downstream of the trailing edge) in order to determine the rise in the mixing losses with downstream distance. Their results included the spanwise variations of the pitchwise-averaged total pressure loss coefficient at several downstream locations. There 
were two distinct loss peaks: first loss peak close to the endwall associated with the endwall boundary layer, and the second loss peak at approximately $10 \%$ span associated with the interaction between the passage vortex and the blade-surface boundary layer. The spanwise location of the second loss peak shifted away from the endwall at farther downstream locations. The inlet boundary layer thickness was varied from $4 \%$ to $22 \%$ of blade span. Increasing the inlet boundary layer thickness was accompanied by a reduction of the corresponding boundary layer shape factor $(H)$ from 1.375 to 1.25. Although the spanwise locations of the loss peaks were not affected by the inlet boundary layer thickness, the magnitude of the second loss peak increased substantially with thickening of the inlet endwall boundary layer. They concluded that the magnitude of the second loss peak was proportional to the boundary layer displacement thickness $\left(\delta^{*}\right)$. However, increasing the inlet endwall boundary layer thickness had no clear effect on secondary losses.

Adjlout and Dixon (1992) investigated the effects of free-stream turbulence intensity $(0.8 \%, 2.2 \%$ and $3.8 \%)$ on endwall losses in a high-aspect-ratio turbine cascade. Increasing the free-stream turbulence intensity resulted in lower midspan blade loadings. The resulting change in profile and secondary losses, however, did not exhibit any clear trend with increased levels of free-stream turbulence intensity.

Gregory-Smith and Cleak (1992) also investigated the effects of free-stream turbulence intensity on secondary flow structures. In their experiments, the free-stream turbulence intensity was increased from $1.4 \%$ to $5 \%$ by using a turbulence generating grid, which also resulted in the reduction of the inlet endwall boundary layer thickness. Increasing the turbulence intensity resulted in higher profile losses $(+7 \%)$ associated with earlier transition on the suction surface of the blade. Within the three-dimensional flow region, higher free-stream turbulence intensity resulted in lower secondary losses $(-18 \%)$ and smaller secondary kinetic energy, which was in part attributed to the thinner inlet boundary layer. They also examined the contribution of $T K E$ production to total pressure losses due to high levels of local turbulence intensity within the passage vortex $(\sim 30 \%)$. Regions of high $T K E$ coincided with regions of high total pressure loss, and the development of TKE and total pressure losses followed similar trends through the passage. The rise in total pressure loss, however, was much larger than TKE production. 
They concluded that significant viscous dissipation effects were necessary to account for the differences between the rise in total pressure loss and TKE production.

The author of this report is not aware of any experimental studies concerning the effects of free-stream turbulence length scale on secondary losses. Increasing the freestream turbulence length scale may result in higher pressure losses due to higher TKE

production. In the current project, the effects of free-stream turbulence intensity and length scale on profile losses are examined numerically in Chapter 5.

In the works of Gregory-Smith et al. (1988b) and Gregory-Smith and Cleak (1992), the turbulence measurements were also used to examine the secondary flow field for flow unsteadiness. An initial spectral analysis of turbulence revealed a discrete frequency of $30 \mathrm{~Hz}$ in a high turbulence region, which was initially attributed to passage vortex unsteadiness. The observed flow unsteadiness was later associated with a large separation bubble on the suction surface of the blade. Data obtained from a similar cascade, however, did not exhibit any dominant frequencies except in regions of very low local turbulence intensity (explained as acoustic resonance by Gregory-Smith and Cleak, 1992). The spectral analysis of the turbulence measurements presented by Adjlout and Dixon (1992) showed a discrete dominant frequency of $50 \mathrm{~Hz}$ within the corner vortices. The source of flow unsteadiness could not be identified in this case. The validity of the steady flow assumption for the present study is examined in Chapter 4.

\subsubsection{Zweifel Coefficient and Blade Loading Distribution}

An important objective of the current research is to investigate the effects of midspan turbine blade loading on secondary flows and secondary losses. As mentioned in section 2.4, the Zweifel coefficients selected for the turbine cascades in the current project are 0.8 and 1.0, which would be regarded as high values of loading for HP turbine airfoils. Furthermore, the cascades have been designed using aft-loaded distributions to reduce the secondary losses as predicted by CFD.

Weiss and Fottner (1995) presented data from two highly-loaded cascades with the same Zweifel coefficient, but different loading distributions as shown in Figure 2.4. The front-loaded cascade, T104, exhibited a higher cross-passage pressure gradient near the leading edge of the blade, which resulted in stronger secondary flows in the forward part 
of the passage. The results for $\mathrm{T} 104$ at three different axial locations within and downstream of the passage revealed a stronger passage vortex and higher secondary losses than for the aft-loaded cascade, T106. The higher secondary losses $(+20 \%)$ were attributed to the dissipation of the higher $S K E(+35 \%)$ produced by T104. The flow angle distributions at the outlet of the cascades were also altered by the midspan blade loading distributions. The results from T106 showed smaller flow underturning associated with a weaker passage vortex.

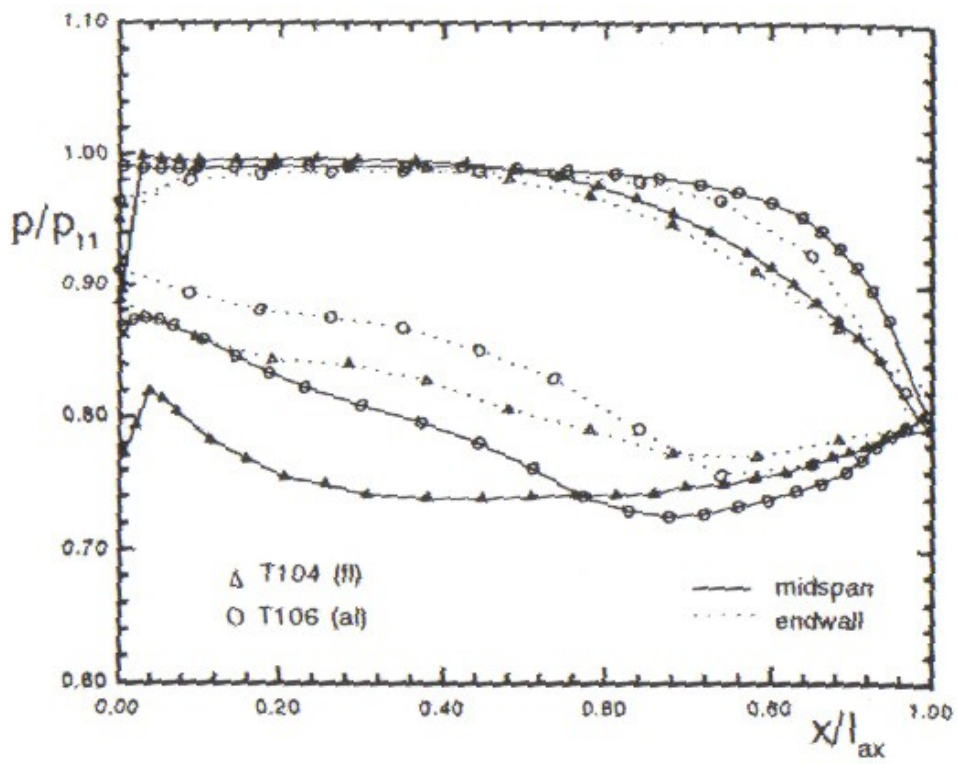

Figure 2.4 Endwall unloading results: front-loaded versus aft-loaded distributions (reproduced from Weiss and Fottner, 1995)

Perdichizzi and Dossena (1993) and Dossena et al. (2004) investigated the effects of blade loading on secondary losses in high speed turbine cascades. Higher blade loadings resulted in higher profile and secondary losses and larger exit flow angle variations (larger underturning and overturning), which may also deteriorate the performance of the subsequent blade rows.

Zoric (2006) investigated the effects of Zweifel coefficient and blade loading distribution on secondary losses in low speed cascades. Three linear turbine cascades with the same inlet and outlet flow angles were used for her study:

- $\quad$ PAKB (aft-loaded): $Z w=1.08$

- PAKD-A (aft-loaded): $Z w=1.37$

- PAKD-F (front-loaded): $Z w=1.37$ 
At 4\% inlet free-stream turbulence intensity, both PAKB and PAKD-A produced higher profile losses than PAKD-F. The higher profile losses were attributed to the presence of separation bubbles on the suction surfaces of PAKB and PAKD-A airfoils, which are common for aft-loaded LP turbine blades. Furthermore, PAKD-A produced significantly higher profile losses than PAKB $(+17 \%)$ associated with the higher Zweifel coefficient $(+27 \%)$. There were significant differences between the secondary flow structures of the three cascades. PAKD-F generated higher secondary losses than PAKB $(+39 \%)$ and PAKD-A (+50\%). The higher secondary losses were accompanied by higher streamwise vorticity and higher $S K E$, congruent to the findings of Weiss and Fottner (1995). PAKD-A produced smaller secondary losses (-7\%) and lower SKE (-9\%) than PAKB, which was not expected due to the higher Zweifel coefficient of PAKD-A. The higher secondary losses produced by PAKB were attributed to the interaction between the large separation bubble on its suctions surface and the secondary flow structures (passage vortex). It may be concluded that the blade loading distribution should be optimized across the span to minimize both profile and secondary losses, which is sometimes referred to as the mixed-loading approach (Knezevici, 2011).

Secondary flows generated within the turbine blade passage also affect the blade loading distribution near the endwalls due to the interaction between the vortical structures and the blade-surface boundary layers. This is commonly referred to as endwall unloading as shown in Figure 2.5, reproduced from Harrison (1990).

The amount of work extracted from a rotor blade is affected by the spanwise variation of the blade loading distribution. In this regard, stronger secondary flows not only increase the secondary losses, but may also introduce a performance penalty due to lower work output from the turbine stage (due to lower blade loading near the endwall). Results from Weiss and Fottner (1995), presented in Figure 2.4, illustrate the differences between endwall unloading characteristics of front-loaded and aft-loaded turbine blades. The front-loaded cascade, T104, exhibits aft-loaded characteristics near the endwall due to strong secondary flows in the forward part of the passage $\left(0<\mathrm{x} / \mathrm{l}_{\mathrm{ax}}<0.4\right)$.

The effects of Zweifel coefficient and blade loading distributions on secondary flows and secondary losses are investigated in the current study, and presented in Chapter 7. 


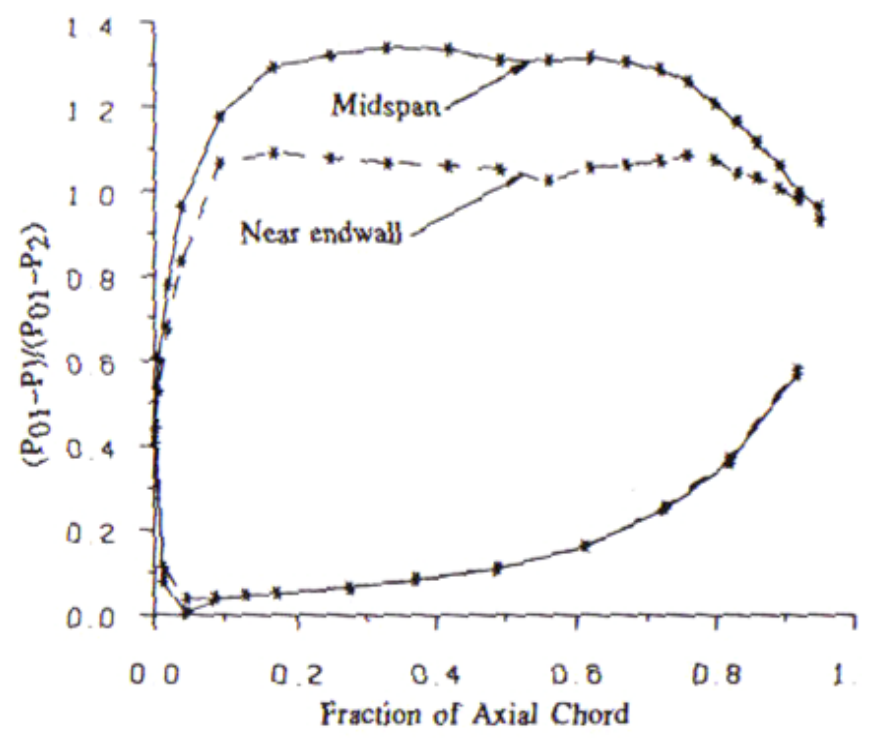

Figure 2.5 Turbine blade loading distribution: midspan loading versus endwall loading (reproduced from Harrison, 1990)

\subsubsection{Total Flow Turning}

In the present study, the effects of total flow turning on secondary losses are also investigated and presented in Chapter 7. The total flow turning values selected for the cascades in the present study are $90^{\circ}$ and $112^{\circ}$.

Yamamoto presented experimental data from two linear turbine cascades: a stator case with low flow turning of $65^{\circ}$ (Yamamoto, 1987a) and a rotor case with high flow turning of $102^{\circ}$ (Yamamoto, 1987b). In the stator case, losses increased slightly inside the passage from $0.75 C_{X}$ to the trailing edge plane. This was attributed to the action of the passage vortex on the suction-surface boundary layer in the aft part of the passage. The results from the rotor case, on the other hand, showed substantial increase in losses inside the passage from $0.25 C_{X}$ to the trailing edge plane. Higher total pressure losses were accompanied with higher levels of $S K E$ inside the passage, associated with stronger vortical structures. SKE decreased substantially downstream of the trailing edge plane as the vortical structures mixed out. The passage vortex was more intense (higher peak vorticity) and wider downstream of the rotor blade. Overall, the rotor cascade produced higher losses at all measurement planes, within and downstream of the blade passage. The author concluded that the interaction between the passage vortex and the suctionsurface boundary layer had the largest contribution to total pressure losses. 


\subsubsection{Flow Compressibility}

The design outlet Mach number for the cascades in the present study is 0.8 . Additional off-design tests were performed for outlet Mach numbers of 0.65 and 0.91 . The pertinent literature on the effects of flow compressibility on secondary flows and losses are presented here.

Measurements in transonic linear turbine cascade facilities have been obtained mainly using pressure probes in the past. Due to potential probe interference effects, such as flow blockage inside the passage, the majority of the measurements have been obtained only upstream and downstream of the cascades. Flow visualization techniques, such as surface oil film visualization (Camus et al. 1984; Duden and Fottner, 1997), and nonintrusive measurement techniques, such as laser Doppler anemometry and Schlieren photography (Michelassi et al., 1998), have been utilized to investigate the flow field structures inside the blade passage. Additionally, CFD has been used to provide complementary data, which may not be easily obtained from the experiments.

Sieverding and Wilputte (1981) investigated the effects of flow compressibility on secondary losses, with and without cooling flows, in a linear turbine cascade with lowaspect-ratio blades $(\operatorname{span} /$ chord $=0.83)$. Three subsonic outlet Mach numbers were investigated: $0.1,0.6$ and 0.8. Raising the outlet Mach number resulted in the migration of the vortical structures towards the endwall of the cascade. This was accompanied by higher flow underturning at 30\% span and lower flow overturning near the endwall. The pitchwise-averaged loss distributions were very similar for the three outlet Mach numbers; the highest Mach number resulted in a slightly higher loss peak associated with a stronger passage vortex. Overall, increasing the Mach number did not have a large influence on secondary losses. They concluded that in the subsonic flow regime, the effects of cooling flows on secondary losses were more important than the effects of flow compressibility.

Bassi et al. (1989) presented both experimental and computational data obtained from a turbine rotor cascade at three exit Mach numbers: 0.5, 1.02 and 1.38. Exit flow angle variation and loss distribution results supported the data obtained by Sieverding and Wilputte (1981). Bassi et al.'s results also indicated a reduction in $S K E$ at higher Mach

numbers. The computational investigations were carried out using a three-dimensional 
Euler (inviscid) code. The numerical simulations overpredicted the strength of the vortical structures downstream of the cascade due to lack of viscous dissipation, but captured the correct trends (e.g. SKE reduction with Mach number).

Perdichizzi (1990) also investigated the effects of flow compressibility on secondary losses in both subsonic and supersonic flow regimes (outlet Mach number $=0.2$ to 1.55). At higher outlet Mach numbers, the passage vortex migrated towards the endwall, and the counter vortex and the corner vortices occupied a larger portion of the flow field. This was accompanied by a reduction of flow underturning at approximately $20 \%$ span and flow overturning near the endwall. In the subsonic flow regime, both secondary losses and profile losses decreased at higher outlet Mach numbers. The loss increase in the supersonic flow regime was mainly attributed to the shock waves.

Perdichizzi et al.'s results (1990) also indicated larger primary kinetic energy (PKE) dissipation, and smaller TKE production at higher Mach numbers. In Perdichizzi's experiments, $S K E$ decay did not fully account for the increase in total pressure losses in the transonic and supersonic flow regimes: the increase in total pressure losses, from $10 \%$ to $110 \%$ axial chord downstream of the trailing edge, was eight times higher than $S K E$ dissipation at an outlet Mach number of 1.02 .

Duden and Fottner (1997) also investigated the effects of flow compressibility and Reynolds number variation on secondary flows for two highly loaded turbine cascades. Three outlet Mach numbers were investigated: 0.3,0.6 and 0.8. Both cascades exhibited more aft-loaded midspan blade loading distributions at higher Mach numbers. Increasing the outlet Mach number from 0.6 to 0.8 resulted in higher profile losses $(+4 \%)$ and lower secondary losses $(-12 \%)$. The authors attributed the reduction in secondary losses to the more aft-loaded blade loading distribution obtained at Mach 0.8. Reducing the Reynolds number from 500,000 to 129,000 at Mach 0.6 resulted in a laminar separation bubble on the suction surface of the blade. Both profile and secondary losses increased accordingly. However, the spanwise distribution of flow angles remained unaltered.

Secondary losses increase downstream of the trailing-edge plane as a result of endwall boundary layer growth and vortex dissipation (Hodson and Dominy, 1987). The contribution of $S K E$ decay to the downstream growth of secondary losses is examined in the present study, as discussed in Chapters 7 through 9. 


\subsection{Control of Secondary Flows}

\subsubsection{Introduction}

Recent turbine research has been focused on reducing the number of turbine blades by increasing the blade loadings. As a result, turbine designers have been investigating methods for reducing the higher secondary losses associated with higher blade loadings. These methods typically utilize endwall modifications, such as endwall fences or endwall contouring, to reduce the growth of the vortical structures inside the passage. Application of endwall fences to restrict the migration of the passage vortex towards the suction surface of the adjacent blade (shown in Figure 2.6) have been investigated by a number of researchers including Chung et al. (1991), Chung and Simon (1993) and Aunapu et al. (2000). These studies also looked at the effects of endwall fences on film cooling effectiveness. They observed enhanced blade surface and endwall cooling due to weaker interaction between the passage vortex and endwall cooling flows. The durability of the fence itself, however, has been a major concern due to high heat transfer rates within the HP turbine stage.

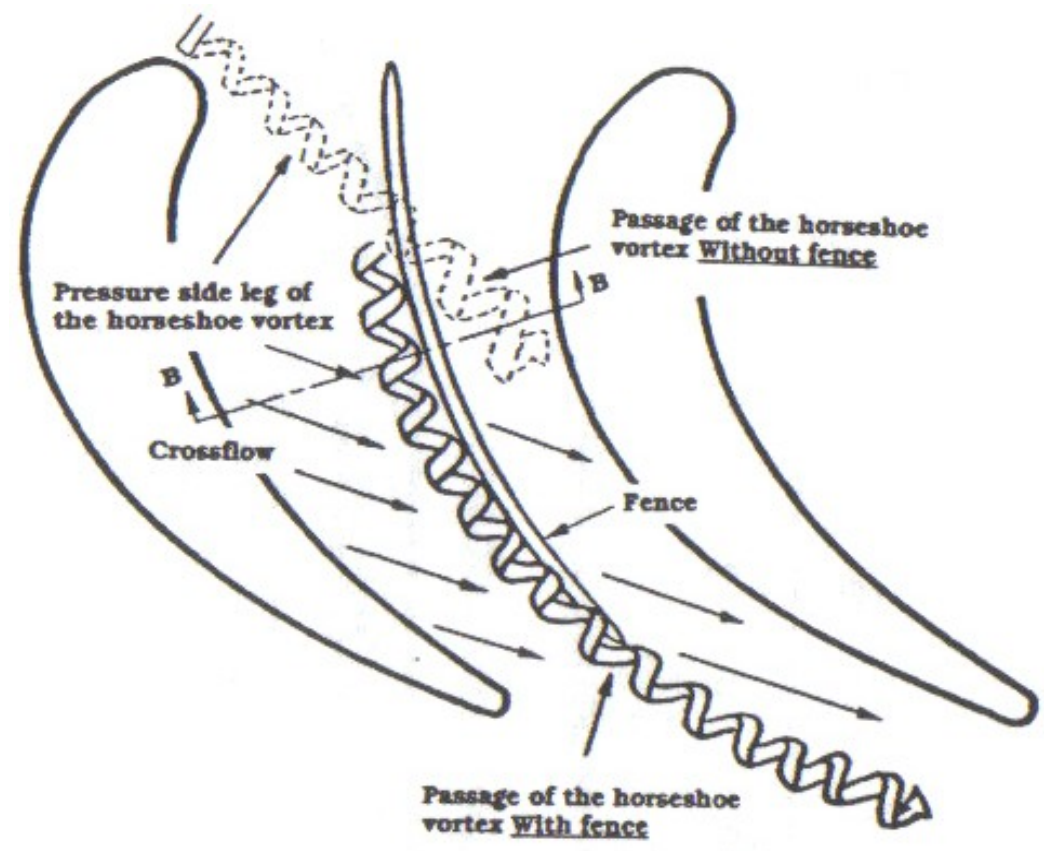

Figure 2.6 Path of the passage vortex with and without the endwall fence (reproduced from Chung et al., 1991) 
In the present study, two different techniques for secondary loss reduction are investigated that are particularly relevant for the more highly loaded cascades; the methods are referred to as non-axisymmetric endwall contouring and airfoil pressure-side modification, as shown in Figure 2.7. The contoured endwalls have been designed by PWA, and the design methods have been described by Praisner et al. (2007). The contoured endwall geometries were optimized using PWA's in-house CFD code (3D RANS code with $k$ - $\omega$ turbulence model) with the goal of minimizing the mass-averaged total pressure losses. The pressure-side modified airfoil has also been designed by PWA.
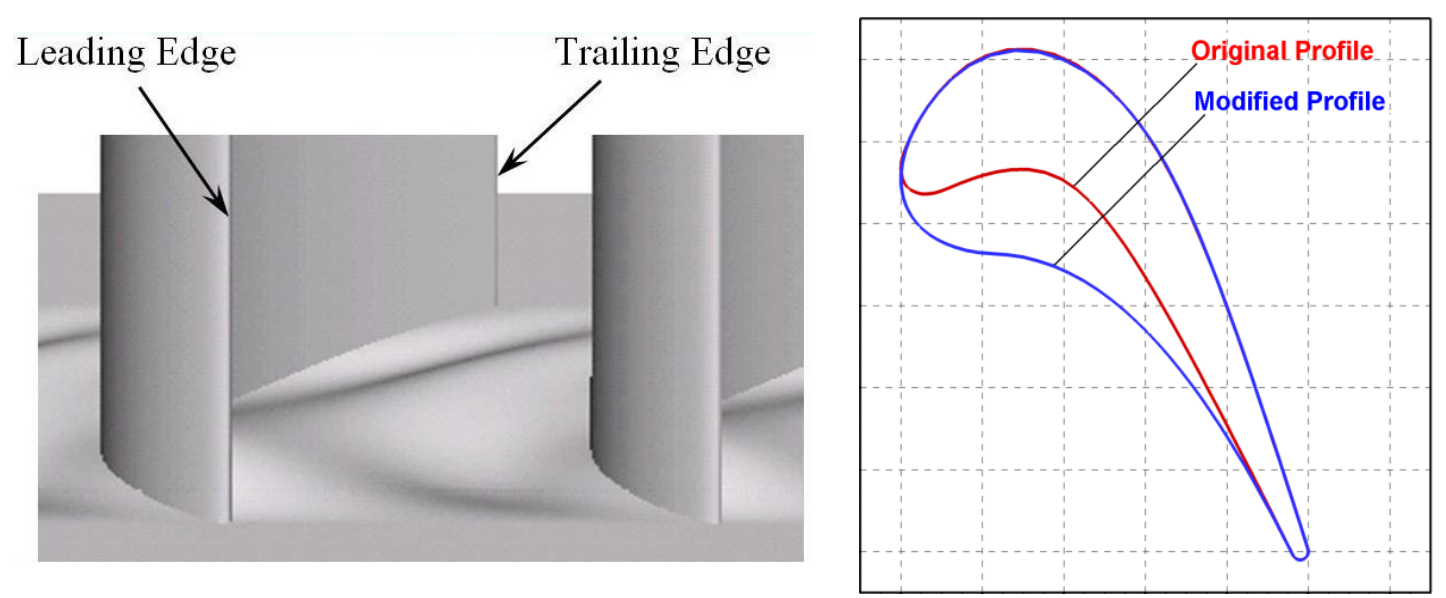

Figure 2.7 Turbine endwall contouring (left) and airfoil pressure-side modification (right)

The main objective of endwall contouring is to reduce the cross-passage pressure gradient by altering the static pressure distribution near the endwall region of the cascade. This is accomplished through streamline curvature by locally accelerating and decelerating the flow over convex and concave regions on the endwall, respectively. By reducing the cross-passage pressure gradient, endwall contouring is aimed at reducing the growth of the passage vortex, which is typically the main source of secondary losses. The airfoil pressure-side modification aims to reduce the flow deceleration in the stagnation region near the leading edge, to delay the formation of the passage vortex. The thicker airfoil profile shown in Figure 2.7 is not expected to increase the airfoil weight since HPT blades typically incorporate internal cooling passages and therefore are hollow. Furthermore, the pressure-side modified airfoil may be optimized to reduce its thickness while preserving the sought-after loading distribution. Previous work on endwall contouring and airfoil profile modification is reviewed next. 


\subsubsection{Endwall Contouring Research}

The effects of endwall contouring on secondary losses have been investigated in both low-speed and high-speed linear turbine cascade facilities in the past. Duden et al. (1999) applied axisymmetric endwall contouring and three-dimensional airfoil design to a highly loaded turbine cascade, with an outlet Mach number of 0.6 , to mitigate the growth of the passage vortex. Their goal was to reduce the cross-passage pressure gradient near the endwall of the cascade. Endwall contouring was also aimed at reducing the adverse streamwise pressure gradient past the suction peak inside the passage. Three-dimensional airfoil design was utilized to produce a radial pressure gradient towards the endwall of the cascade, which was intended to reduce the migration of the passage vortex towards the midspan. Endwall contouring significantly reduced the strength of the passage vortex. They observed a $26 \%$ reduction in secondary losses. Flow underturning and overturning were also significantly reduced for the contoured case. The reduction in secondary losses, however, was accompanied by higher profile losses $(+7 \%)$ due to higher midspan blade loadings.

Dossena et al. (1999) also investigated the effects of axisymmetric endwall contouring on secondary losses under compressible flow conditions. The growth of the passage vortex was inhibited by stronger flow acceleration over the contoured endwall. Overall, endwall contouring resulted in weaker secondary flow structures and smaller row losses (26\% reduction).

Over the past decade, the research groups at Rolls-Royce plc and Durham University have designed and tested several non-axisymmetric profiled endwalls with the aim of minimizing the secondary kinetic energy of the vortical structures and reducing the exit flow under/overturning. The CFD predictions by Harvey et al. (2000) showed significant reduction of secondary kinetic energy and exit flow angle variation, and a small reduction in losses due to endwall contouring. Hartland et al. (2000) presented experimental data from the contoured-endwall cascade designed by Harvey et al. (2000). Endwall contouring resulted in a reduction of the endwall cross-passage pressure gradient in the forward part of the passage. This was accompanied by a significant reduction of secondary flows through the passage, resulting in a less intense, but wider passage vortex within and downstream of the cascade. The passage-vortex-induced counter vortex also 
appeared smaller and less intense due to weaker interaction between the passage vortex and the suction-surface boundary layer. The downstream measurements revealed a strong corner vortex produced by the contoured endwall cascade. The corner vortex resulted in a significant increase in $S K E(+50 \%)$ at the spanwise location closest to the endwall. Flow overturning near the endwall, however, was not affected by the corner vortex. There was a 30\% reduction in secondary losses, which had not been predicted by CFD. They concluded that additional loss reduction could be achieved by decreasing the strength of the corner vortex. Further investigations in a cold-flow rotating test rig substantiated the improvements due to endwall contouring downstream of the blade rows (Rose et al., 2001; Harvey et al., 2002).

Ingram et al.'s results (2002) showed similar trends in terms of secondary loss reduction due to endwall contouring. It was also confirmed that the loss reductions were not appreciably influenced by the transition behaviour on the endwalls (Ingram et al., 2005a). In one case, however, Ingram et al.'s experimental results (2005b) revealed large endwall separation, not predicted by CFD, for an "aggressive" contouring design, which ultimately led to higher losses albeit with smaller $S K E$.

More recently, Knezevici (2011) presented low-speed experimental results from an aft-loaded cascade (PAKB) and a front-loaded cascade (PAKD-F) with different Zweifel coefficients. The application of endwall contouring resulted in secondary loss reductions for both cases: $15 \%$ for PAKB and $32 \%$ for PAKD-F. The measured improvements in loss, which exceeded the numerical predictions, were mainly attributed to the diminished size and strength of the passage vortex, reducing the associated free-stream flow convection rates. This was also accompanied with smaller $S K E$ and flow underturning, subsequently recognized as additional benefit in terms of the mixed-out losses.

Other studies from low-speed cascades (Gustafson et al., 2007; Torre et al., 2011) and model test rigs (Schuepbach et al., 2009; Germain et al., 2010) have also demonstrated the effectiveness of endwall contouring for both low- and high-pressure turbines. The effects of endwall contouring on secondary losses in high-speed linear cascades are investigated in the current study, as presented in Chapter 8. 


\subsubsection{Airfoil Pressure Surface Modification}

The modification of airfoil pressure surface to reduce the secondary losses in turbines is a novel design approach. Therefore, the available literature on the subject is very limited at this point.

The application of leading edge bulbs to the T106 airfoil (Figure 2.4) was examined by Sauer et al. (2001). The resulting configurations are labelled T106/1 and T106/2 in Figure 2.8. They observed a 50\% reduction in losses associated with a weaker passage vortex. The intensification of the suction-side leg of the horseshoe vortex was credited with delaying the migration of the passage vortex towards the suction side of the passage.

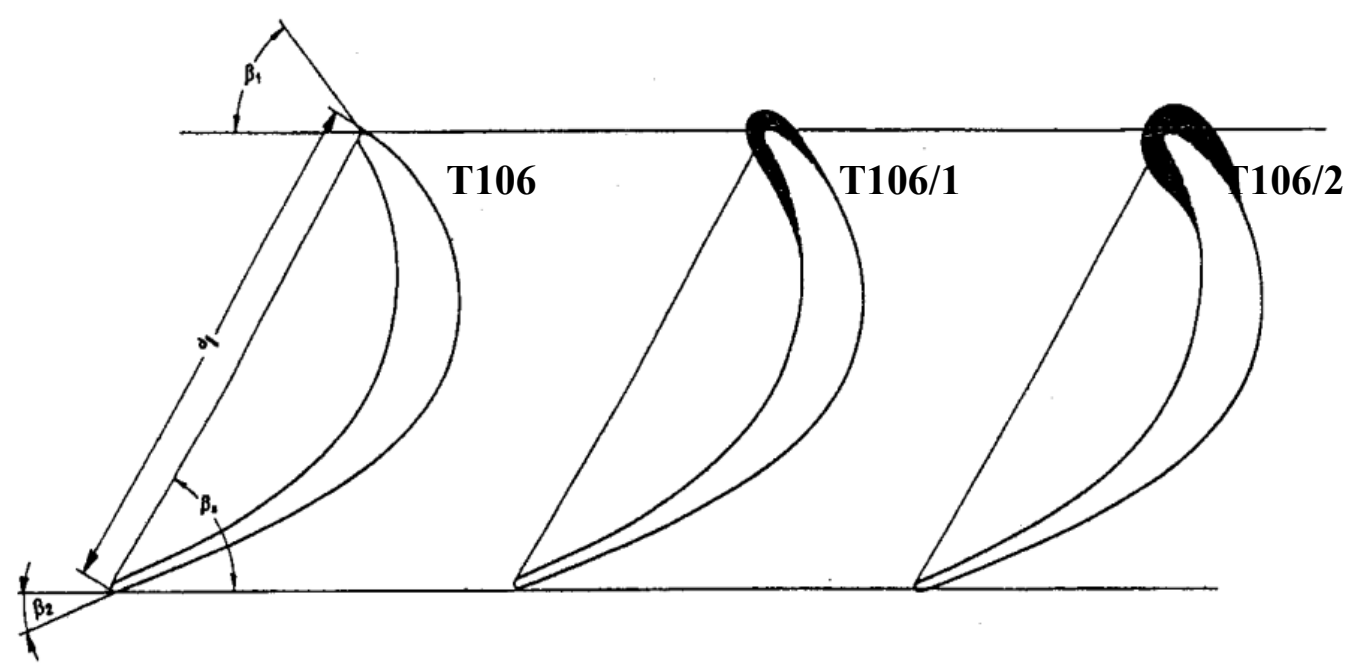

Figure 2.8 Application of leading edge bulbs to the T106 airfoil (reproduced from Sauer, 2001)

Brear et al. (2002) presented results from four turbine airfoils with identical suction surface geometry and different pressure surface geometries, as shown in Figure 2.9. The goal of the study was to suppress a pressure-side separation bubble that was present on the original airfoil (labelled "A" in Figure 2.9). However, the secondary flows and losses were also affected by the pressure-side modification. Increasing the thickness of the airfoil resulted in smaller adverse pressure gradients, which in turn eliminated the separation bubble on the pressure surface. Peak flow underturning was also reduced by $2^{\circ}$. The consequent $10 \%$ reduction in mass-averaged row losses for Airfoil D, however, was primarily attributed to the suppression of the separation bubble by the authors. 


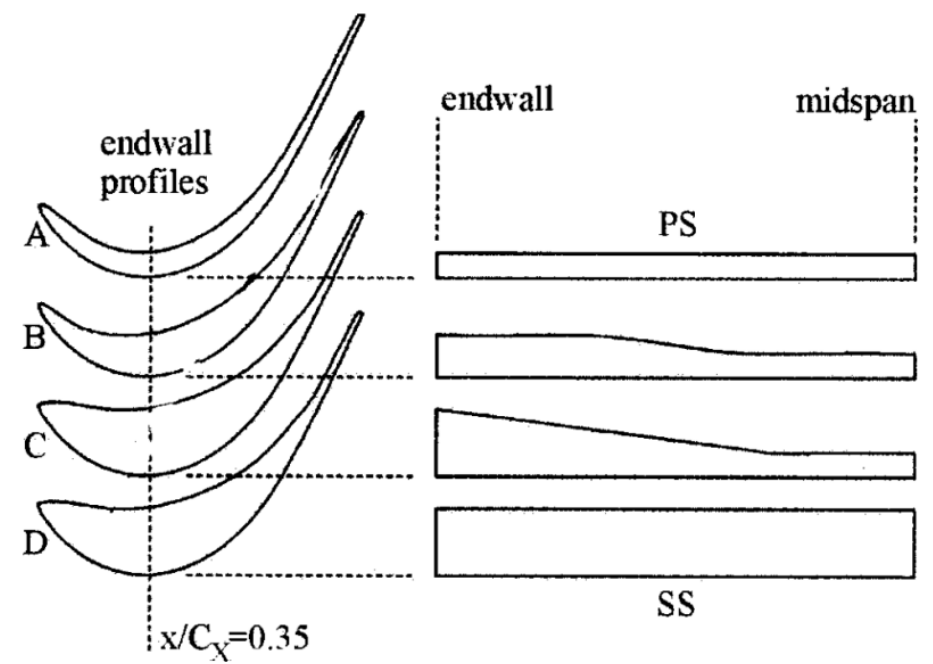

Figure 2.9 Modification of the airfoil pressure-side profile (reproduced from Brear, 2002)

As mentioned, the modification of the airfoil pressure-surface profile in the present study is mainly aimed at reducing the secondary losses. A related study was recently completed by Knezevici (2011) using low-speed linear turbine cascades. The pressure side modification was applied to the PAKD-F airfoil, resulting in the PAKD-FP configuration. In comparison to PAKD-F, the PAKD-FP cascade showed weaker crossflows near the endwall and lower vorticity associated with a weaker passage vortex. Consequently, the secondary losses were reduced by $44 \%$ for PAKD-FP. The results suggest that the pressure-side modification is a viable method for reducing the secondary losses in turbines. The effects of pressure-side modification on secondary losses in transonic flows are investigated in the current project, as discussed in Chapter 9. 


\section{Chapter 3}

\section{Experimental Apparatus and Measurement Procedures}

\subsection{Introduction}

The experimental investigations were conducted in the Pratt and Whitney Canada (PWC) High-Speed Wind Tunnel Laboratory at Carleton University. The wind tunnel, shown schematically in Figure 3.1, was commissioned by Jeffries (1994) and has been used extensively for midspan flow measurements in the past. Various high-pressure and low-pressure turbine cascades have been examined to study the effects of flow compressibility and off-design incidence on turbine profile aerodynamic performance (Islam, 1999; Jeffries, 2000; Jouini, 2000; Corriveau, 2005). The components of the wind tunnel laboratory have been described in detail by Corriveau (2005). Therefore, only the most relevant features of the wind tunnel and instrumentation are summarized here for completeness. The focus of this chapter is on the recent modifications and upgrades to the test section and the data acquisition system; these include the replacement of the data acquisition hardware and software, three-dimensional flow measurements using a seven-hole pressure probe and a new probe calibration rig. The turbine cascades employed for the current study, the experimental instrumentation and the measurement procedures are also described here.

\subsection{PWC High-Speed Wind Tunnel Laboratory}

\subsubsection{Main Features of the Wind Tunnel}

The high-speed wind tunnel is of the blow-down type with typical run durations of approximately 40 seconds. A Broom Wade VC500 two-stage reciprocating compressor is 


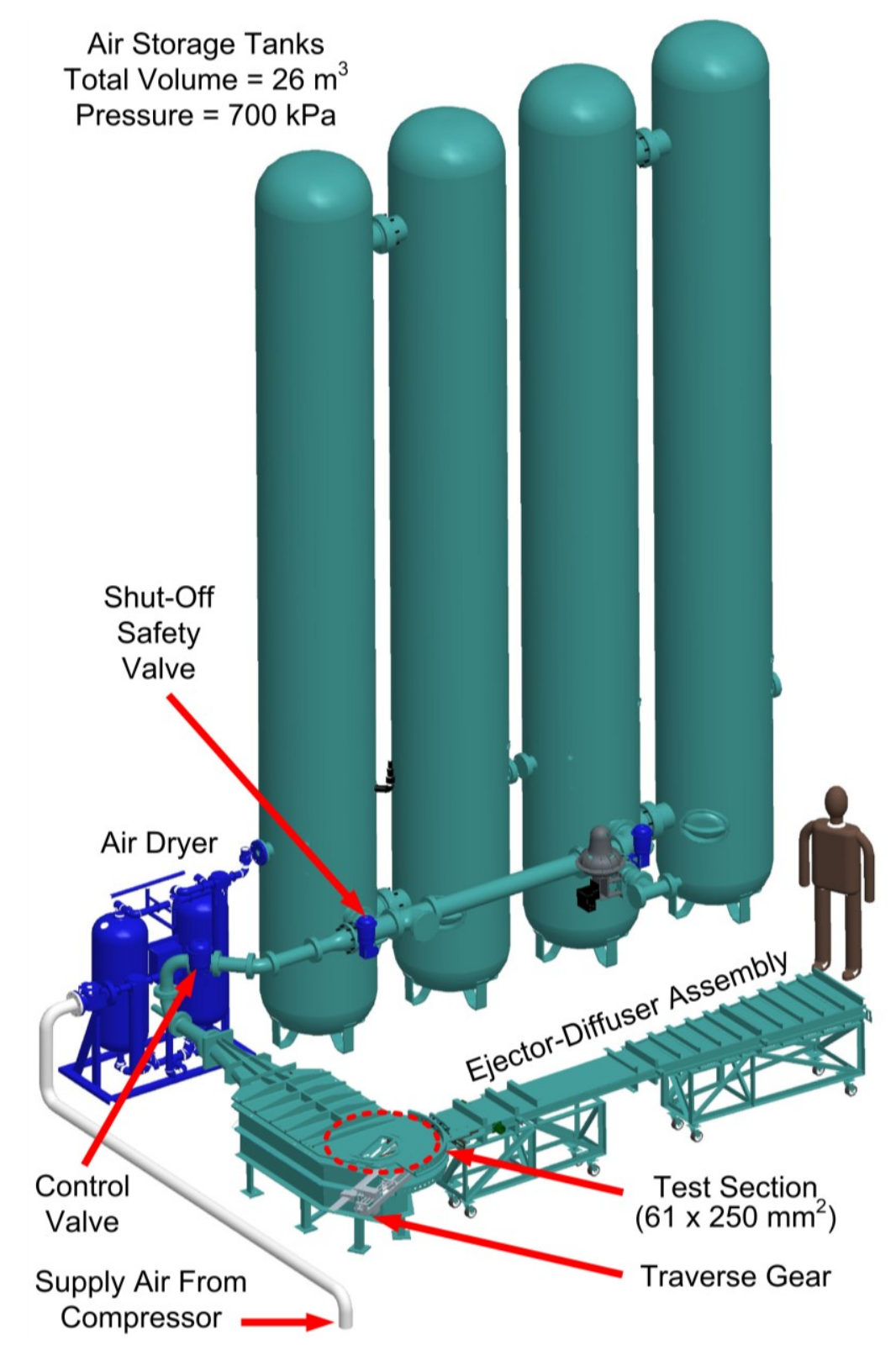

Figure 3.1 High-Speed Wind Tunnel Laboratory at Carleton University

used to fill the storage tanks (total volume $\approx 26 \mathrm{~m}^{3}$ ) with pressurized air at about 800 $\mathrm{kPa}(\mathrm{a})$. The compressed air is cooled using an intercooler within the compressor and an aftercooler following the high-pressure stage. The moisture in the air is removed by a Comp-Air Kellogg CDH520 regenerative desiccant dryer prior to storage in the tanks. In this process, air dewpoint is decreased to about $-45^{\circ} \mathrm{C}$ to avoid any condensation and icing in the test section. It takes approximately 20 minutes to raise the pressure in the tanks, monitored using an OMEGA PX613 pressure transducer, from atmospheric to about $800 \mathrm{kPa}(\mathrm{a})$. Once the tanks are fully pressurized, air is first discharged through a 
shut-off safety valve (Neles-Jamesbury $Q P 3 C-M$ actuator coupled to an $815 L$ 6" butterfly valve), and then throttled using an electro-pneumatic control valve (Neles-Jamesbury $Q P 2 C / M$ actuator coupled to a $71504 "$ ball valve). The throttle valve is controlled using a feedback loop to keep the tunnel blowing pressure and thus the Mach number approximately constant. The Mach number at the outlet of the turbine cascade can be varied from about 0.3 to 1.4 depending on the total throat area of the cascade. The Reynolds number in the test section can be set independently of the Mach number through use of the ejector-diffuser assembly. The ejector nozzles, however, were not employed for the present study (Reynolds number varied with Mach number) due to the limited range of Reynolds number encountered. The use of the diffuser, on the other hand, allowed for longer run times by reducing the static pressure at the outlet of the cascade ( $80 \mathrm{kPa}(\mathrm{a}))$ as compared to atmospheric conditions. During the run, the air is discharged into the laboratory through the diffuser.

\subsubsection{Wind Tunnel Test Section}

The general configuration of the test section is shown schematically in Figure 3.2. The linear cascade consists of several airfoils, a suction-side endblock and a pressure-side endblock, mounted on an endplate. The cascade is mounted on a turntable, allowing for inlet incidence variation. Once the turntable is rotated and set to the desired incidence, the tunnel walls are adjusted to satisfy the flow quality requirements. The sliding walls and the test section side walls are adjusted to establish inlet flow uniformity; the adjustments are made based on the inlet flow angle and the distance between the two endblocks. Downstream of the cascade, the transition walls are aligned in the direction of the airfoil outlet metal angle. The probe traverse mechanism is also attached to the outer transition wall, and thus the probe holder is set parallel to the transition walls. A Lexan window covers and seals the test section. Sample results for inlet flow uniformity and outlet flow periodicity are presented in Section 3.9.

As in previous experiments in this facility (Corriveau, 2005), a turbulence-generating grid is used to raise the inlet turbulence intensity to about $4 \%$ for all the test cases. Various pressure and temperature measurements are collected to document the flow characteristics in the wind tunnel. The reference total temperature $\left(T_{01, \text { ref }}\right)$ and total 
pressure $\left(P_{01, r e f}\right)$ are measured at the upstream end of the test section, before the contraction. Pressure measurements are collected in the test section using Pitot tubes, Kiel probes and multi-hole pressure probes. In addition, the top Lexan window, the bottom endplate, and the two airfoils adjacent to the centre passage are instrumented with surface static pressure taps. These static taps are used to monitor the inlet and outlet static pressures $\left(P_{S 1}\right.$ and $\left.P_{S 2}\right)$ and measure the blade loading distributions, respectively. Additional details regarding the measurement locations and procedures are provided in Section 3.7.

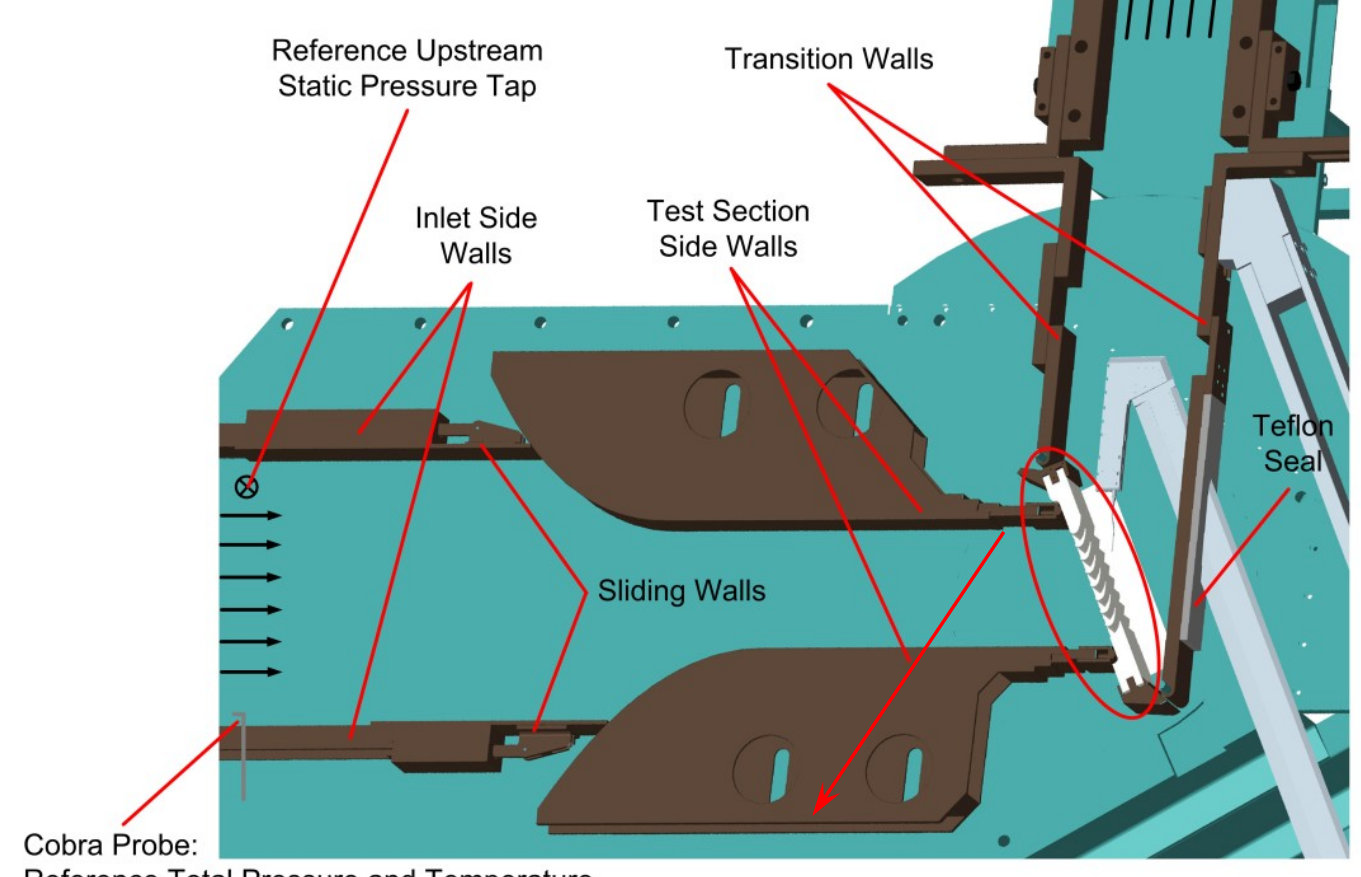

Reference Total Pressure and Temperature

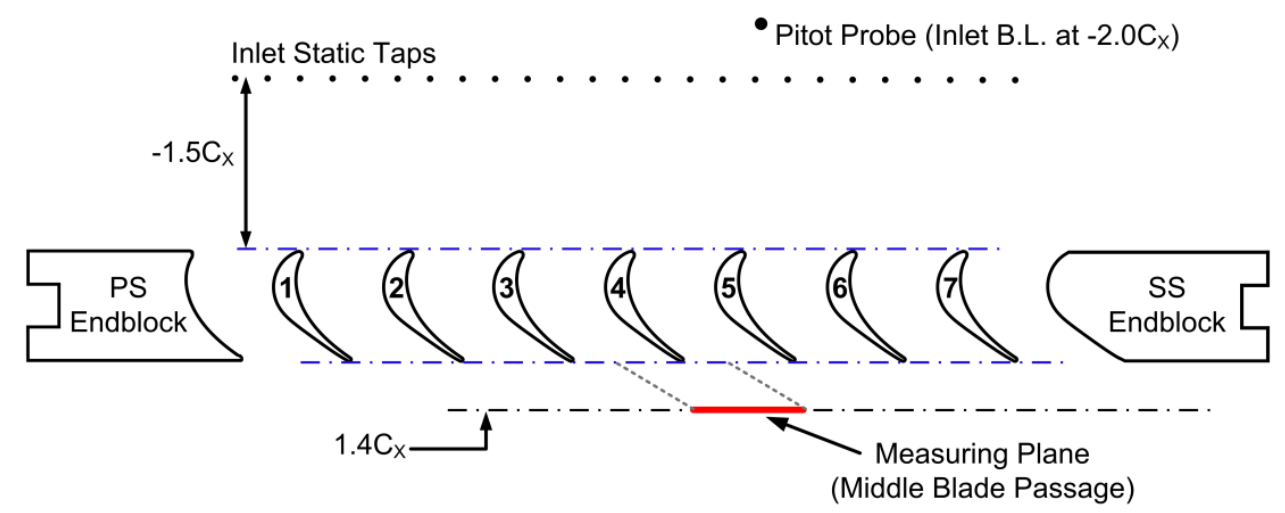

Figure 3.2 Cascade test section 


\subsection{Experimental Instrumentation}

\subsubsection{Data Acquisition System}

As part of the present study, the test section of the wind tunnel was modified to allow for three-dimensional flow measurements using a seven-hole pressure probe. The data acquisition hardware and software were also upgraded to facilitate the seven-hole probe measurements. In the preliminary stages of the experimental investigations, it was found that the pneumatic response of the system was adversely affected by the use of a 48-port Scanivalve SSS/CM48-1 pressure measuring system with a single pressure transducer, which was used by Corriveau (2005) for his measurements using a three-hole pressure probe. Using separate transducers connected to each pressure port on the seven-hole probe, and implementing a $250 \mathrm{~ms}$ delay after moving the probe prior to data collection, eliminated the pneumatic response problems; this was verified by analyzing the step response of the system as discussed in Section 3.7.

The data acquisition system employs three absolute and eight differential Druck pressure transducers as indicated in Table 3.1. Seven transducers are connected to the seven ports of the seven-hole probe, shown in Figure 3.3. The remaining transducers are connected to $P_{01, r e f}, P_{S 1}, P_{S 2}$ and $P_{a t m}$. The atmospheric pressure is collected at every data point in order to extract the absolute pressure from the differential pressure transducers,

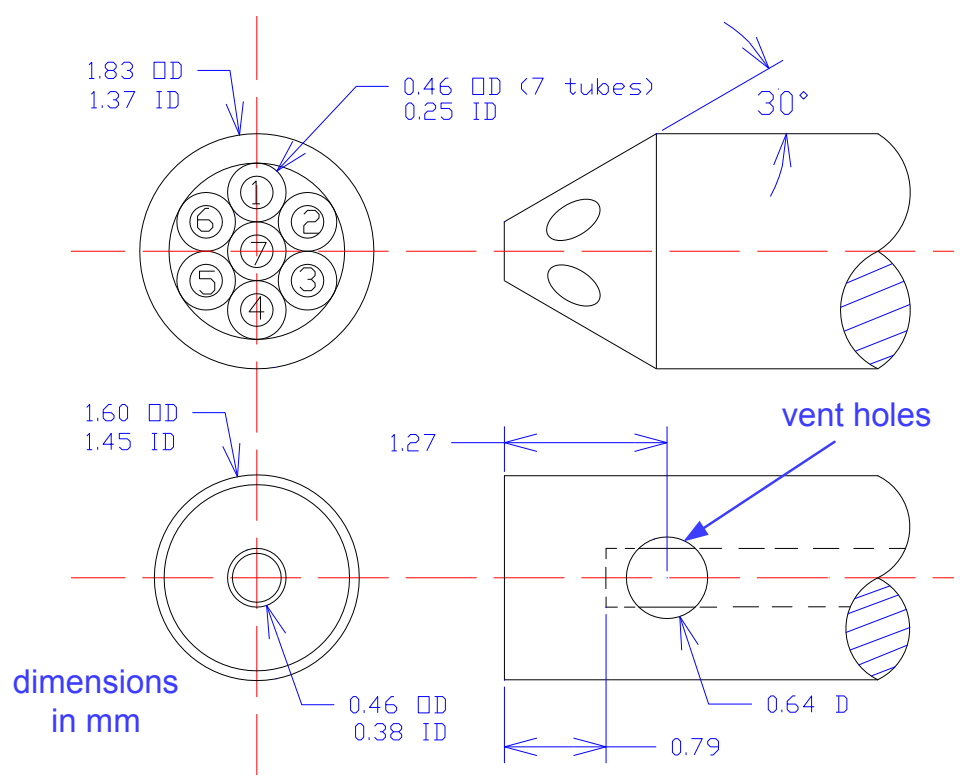

Figure 3.3 Seven-hole probe (top) and Kiel probe (bottom) 
which had their reference ports open to atmosphere. The atmospheric pressure is also monitored using an OMEGA DPI 740 digital barometer for comparisons. The signals from the transducers are amplified and acquired using a 16-bit National Instruments (NI) PCI-6229 data acquisition card (32 analog inputs and a combined sampling rate of 250 $\mathrm{kS} / \mathrm{s}$ ) installed in a Microsoft Windows-based computer. A K-type thermocouple is used to monitor the upstream total temperature. The thermocouple signal is filtered, amplified and acquired using a 24-bit NIUSB-9211A data acquisition system. The data acquisition software was developed using NI LabVIEW 8.0.1.4000.

Table 3.1 Summary of pressure transducers

\begin{tabular}{|c|c|c|c|c|}
\hline Channel & Serial Number & Range $(\mathrm{psi})$ & Type & Pressure Measurement \\
\hline 1 & 76738 & 50 & Absolute & $\mathrm{P}_{\mathrm{atm}}$ \\
\hline 2 & 76741 & 100 & Absolute & $\mathrm{P}_{01, \text { ref }}$ \\
\hline 3 & 134061 & 100 & Differential & $\mathrm{P}_{\mathrm{S} 1}$ \\
\hline 4 & 76740 & 100 & Absolute & $\mathrm{P}_{\mathrm{S} 2}$ \\
\hline 5 & 114370 & 50 & Differential & Seven-hole probe: P7 \\
\hline 6 & $\mathrm{~B} 6228$ & 50 & Differential & Seven-hole probe: P1 \\
\hline 7 & $82842 / 834$ & 50 & Differential & Seven-hole probe: P2 \\
\hline 8 & $\mathrm{~B} 3116$ & 75 & Differential & Seven-hole probe: P3 \\
\hline 9 & 134060 & 100 & Differential & Seven-hole probe: P4 \\
\hline 10 & $\mathrm{~B} 6202$ & 100 & Differential & Seven-hole probe: P5 \\
\hline 11 & B6992 & 100 & Differential & Seven-hole probe: P6 \\
\hline
\end{tabular}

\subsubsection{Pressure and Temperature Probes}

As identified in Figure 3.2, a cobra-head (three-hole) pressure probe with an integrated thermocouple was used to measure the inlet reference total pressure $\left(P_{01, r e f}\right)$ and total temperature $\left(T_{01, r e f}\right)$. The cobra-head probe did not affect the inlet flow uniformity or outlet flow periodicity results, as will be seen later. A separate reference wall static pressure tap was used to control the tunnel blowing pressure (also shown in Figure 3.2). Since the static tap is located upstream of the contraction, where the flow velocity is low, the measured pressure is close to the upstream total pressure.

The inlet total pressure $\left(P_{01}\right)$ is required to determine the pressure losses through the cascade. To obtain the inlet total pressure, the measured reference total pressure was corrected for wind tunnel losses incurred between the cobra probe and the inlet of the 
cascade (Figure 3.2), as measured by a separate Pitot probe (e.g. $P_{01} / P_{01, \text { ref }}=0.995$ at $M_{1}=0.3$ ). The inlet total pressure was also used to normalize all other pressure measurements, and thus to minimize the effects of blowing pressure fluctuations. During the run, the total temperature drops by approximately $20^{\circ} \mathrm{C}$ due to expansion of the air in the tanks. The inlet total temperature is recorded for every data point to determine the air density at the outlet of the cascade, assuming adiabatic expansion (no downstream total temperature measurements).

The inlet boundary layer was traversed using a gooseneck Pitot probe $(D=0.9 \mathrm{~mm})$ at approximately 2.0 axial chords upstream of the cascade. As discussed by Corriveau (2005), the position of the Pitot probe relative to the wall was determined aerodynamically: the minimum total pressure is collected at $0.7 \%$ span, corresponding to the radius of the probe; as the probe stem moves closer to the endwall, the probe tip bends away from the endwall, thus recording higher total pressures. A seven-hole probe and a Kiel probe were used to collect the downstream flow field measurements. Schematic drawings of these probes are shown in Figure 3.3. The Kiel probe has two vent holes located on the outer casing slightly downstream of the inner tube rather than using the more common, straight-through arrangement. The Kiel probe and the sevenhole probe have similar spatial resolutions (ratio of probe diameter to blade span $\approx 0.03$ ).

\subsubsection{Probe Traverse Mechanism}

The downstream probe traverse mechanism is shown in Figure 3.4. The position of the traverse mechanism was adjusted to align the probe with the airfoil outlet metal angle, thus minimizing the flow angles relative to the probe. A step-motor driven traverse gear was used to traverse the probe in the pitchwise direction; this system consists of a custom-built stepping motor controller and a Compumotor stepper motor. Each step moves the probe holder, and thus the probe by about $0.01 \mathrm{~mm}$ in the pitchwise direction, which provides an acceptable resolution relative to the minimum airfoil pitch of $24.6 \mathrm{~mm}$ (Section 3.10). The spanwise position of the probe was set and measured manually with wind off. The axial location of the probe in the probe holder could also be adjusted to allow the measurement plane to be adjusted. Measurements were made at various times 
for the following measurement planes: $20 \%$, 40\% (main measurement plane), $70 \%$ and $100 \%$ axial chords downstream of the trailing edge.

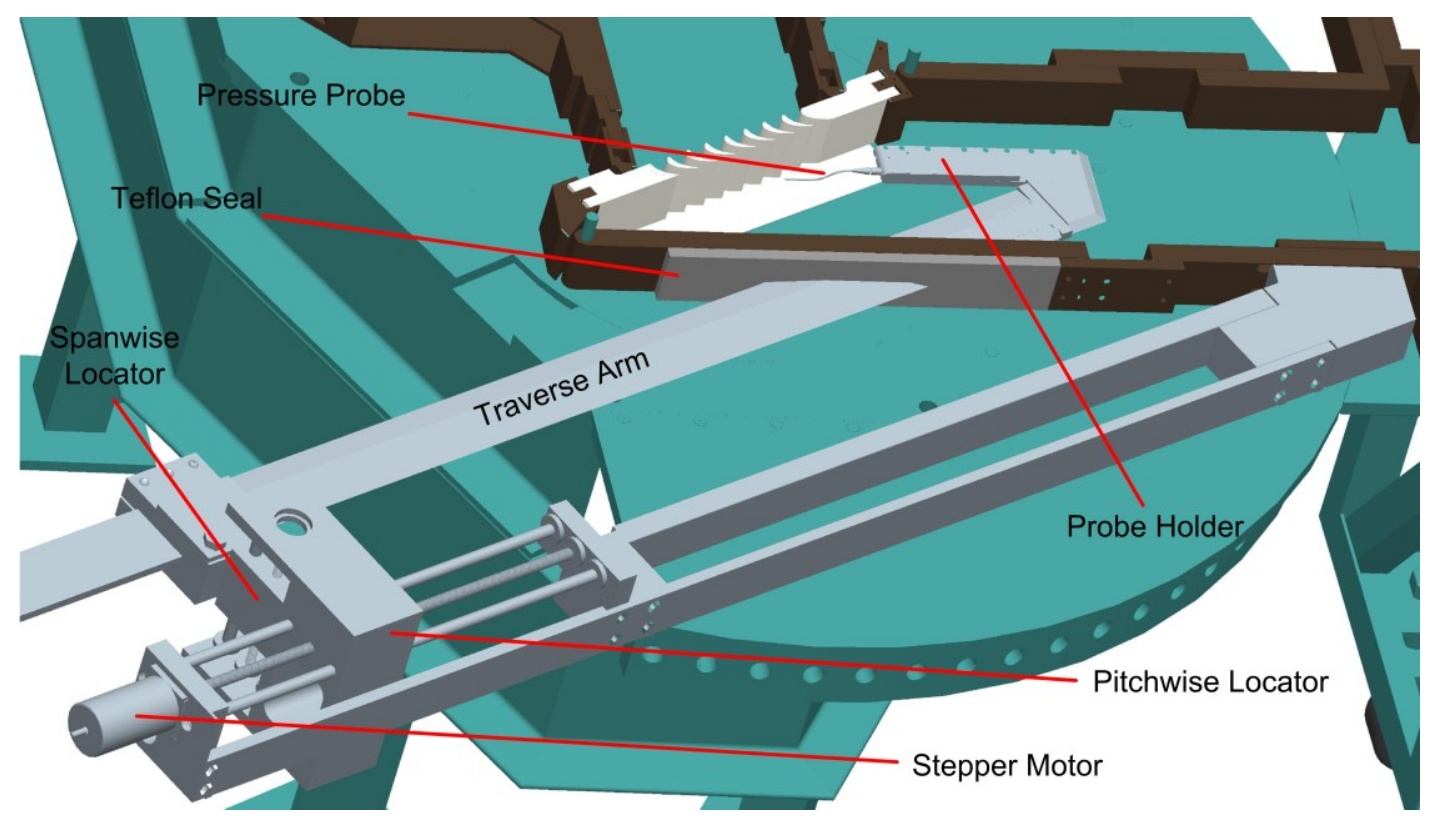

Figure 3.4 Probe traverse mechanism

\subsubsection{Probe Calibration Rig}

Initially, the pressure probes were calibrated in the wind tunnel using a test-section insert, as described by Corriveau (2005). However, this calibration insert was awkward to use, and required the disassembly of the cascade when calibration needed to be performed. A new probe calibration rig was therefore designed and manufactured to increase the density of the calibration grid and reduce the calibration times. The probe calibration rig consists of a settling chamber, a bell mouth inlet and a convergent nozzle, as shown in Figure 3.5 .

The settling chamber is provided with pressurized air from the wind tunnel storage tanks using an electro-pneumatic control valve (Neles-Jamesbury $Q P 1 C / M$ actuator coupled to an $R 212$ " segmented ball valve). Flow from the settling chamber enters the bellmouth inlet and is discharged through the constant-area nozzle to atmosphere where the probe is mounted. The settling chamber and the nozzle outlet are both instrumented with static taps, providing reference total pressure and reference downstream static pressures, respectively. The actual total and static pressures at the probe position have been calibrated against these reference pressures for multiple Mach numbers. 

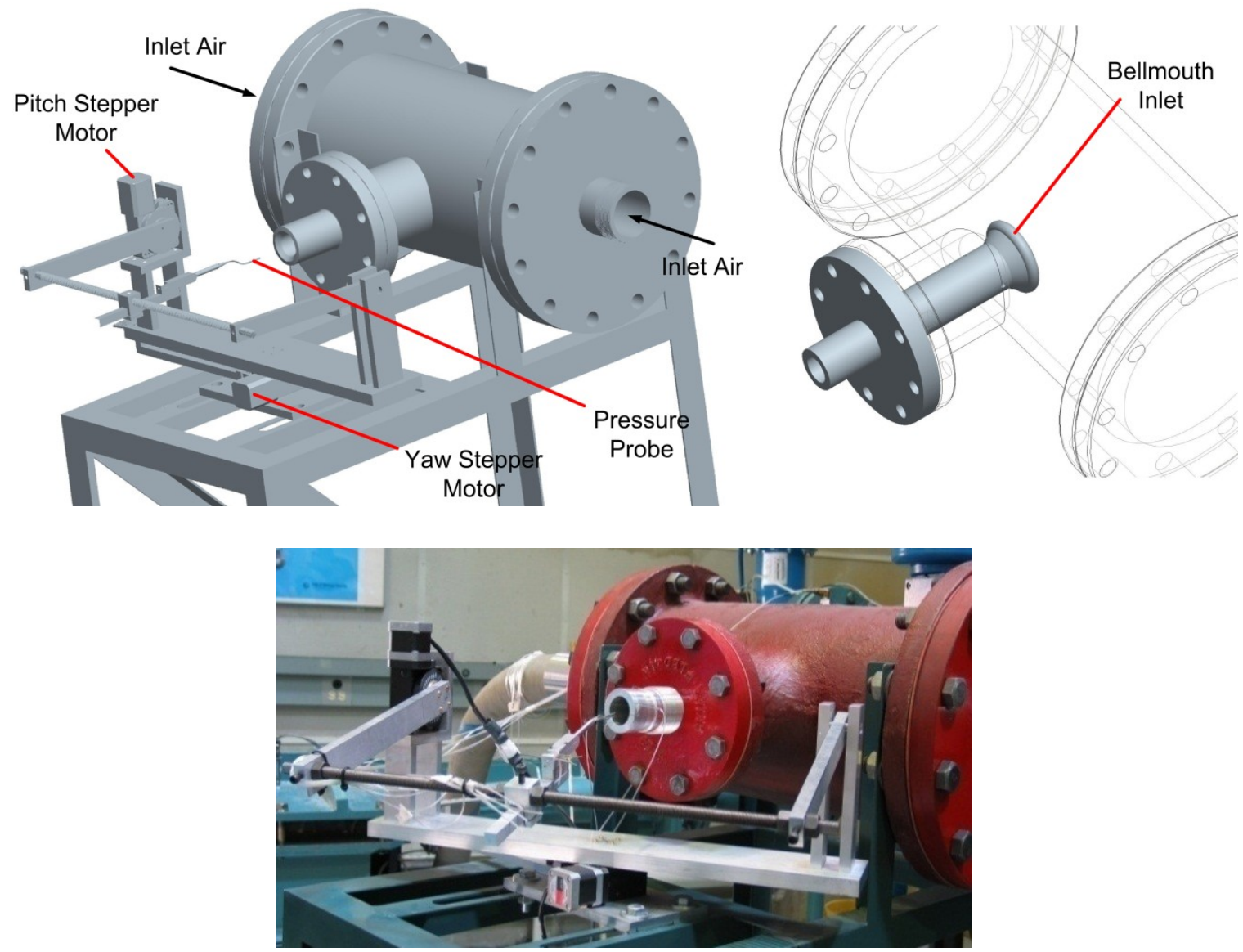

Figure 3.5 Probe calibration rig

The probe is mounted in a support that can be rotated about two axes, pitch and yaw, using stepper motors; this system consists of two VEXTA PK245-01AA stepper motors and a VELMEX VXM stepping motor controller. The stepper motors are capable of $1.8^{\circ}$ steps, geared down to steps of $0.01^{\circ}$. The probe may be rotated in the pitch and yaw directions by up to $\pm 30^{\circ}$. The probe tip is positioned at the centers of rotation, ensuring that its absolute position in the discharging jet is invariant. The discharging jet was characterized extensively by Pitot, static and three-hole probes to verify flow uniformity in both the axial and radial directions (negligible variations in total and static pressures and flow angles). The Mach number of the calibration jet may be varied from about 0.2 to 0.95 . The turbulence intensity of the jet is approximately 5\% (Scribner, 2011), which is slightly larger than the turbulence intensity at the inlet of the cascade (4\%). The probe calibration rig was mainly used to calibrate the seven-hole probe and assess the flow angle sensitivity of the Kiel probe as described in the next sections. 


\subsection{Pressure Transducer Calibration}

Eleven temperature-compensated Druck PDCR 22 pressure transducers, summarized in Table 3.1, were used to collect the pressure measurements. The transducers were kept in a controlled environment to minimize the impact of temperature and pressure drifts in the laboratory. The transducers were calibrated frequently using a high precision digital pressure calibrator (Druck DPI 605), which constitutes a secondary pressure standard. The Druck DPI 605 unit was calibrated against a primary pressure standard by the manufacturer prior to purchase and testing (DPI 605 was used within the three-year period following the manufacturer's calibration date). The supply pressure was varied from about 5 psia to 45 psia, as shown in Figure 3.6, to establish a linear correlation between the pressure and the voltage output of the transducers $(P=m V+b)$. A hysteresis analysis was also performed, which showed negligible impact on the calibration results. Throughout the repeated calibrations, the calibration slope $(\mathrm{m})$ of each transducer remained approximately constant. On the other hand, the calibration intercept $(b)$ of each transducer was updated at the beginning of each test day based on the atmospheric pressure reading. The small fluctuations in transducer zero drift did not increase or decrease substantially over the course of the experiments.

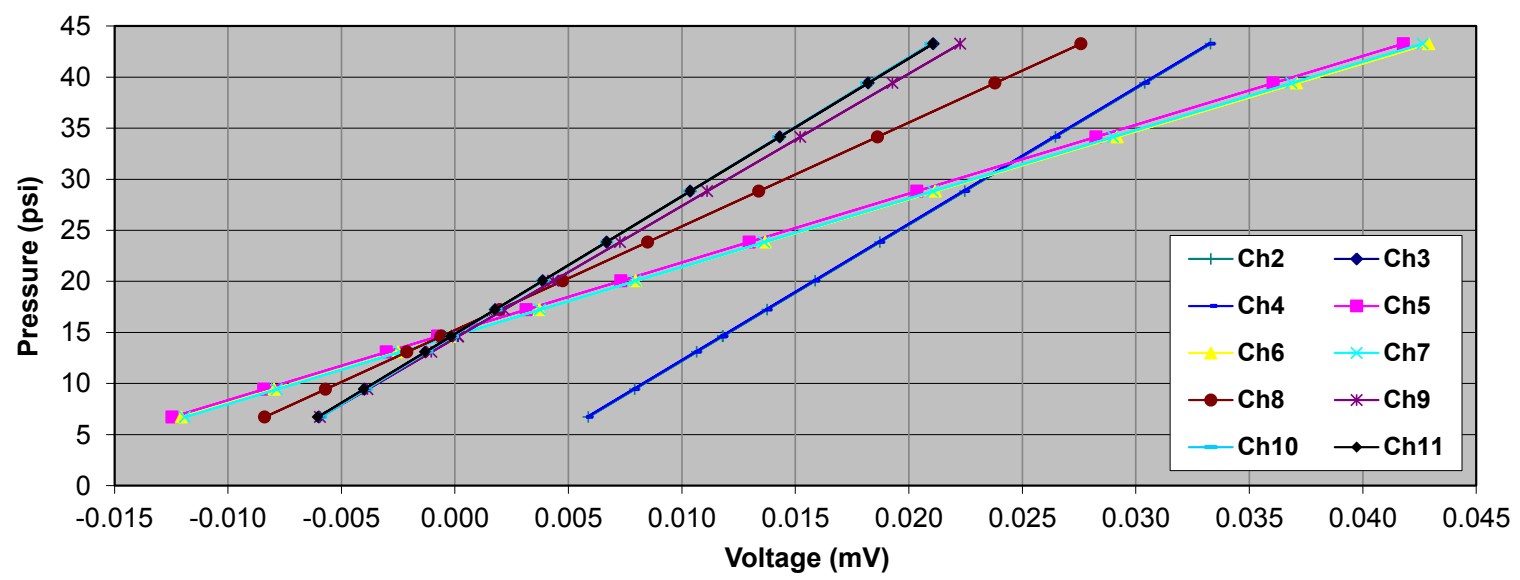

Figure 3.6 Pressure transducer calibration curves

\subsection{Kiel Probe Assessment: Flow Angle Sensitivity}

Kiel probe sensitivity to flow misalignment angles was investigated at two different Mach numbers, as presented in Figure 3.7. The flow misalignment angle $(\varphi)$ is defined as the angle between the incoming flow and the probe center axis. Pressure measurements 
obtained from the centre port of the seven-hole probe are also presented for comparison purposes.

The Kiel probe is insensitive to flow misalignment angles below about $25^{\circ}$. Kiel probe characteristics also seem to be largely Mach number independent in the subsonic range. Similar results for the Kiel probe were obtained in a low speed (incompressible) probe calibration rig (Scribner, 2011). The centre port of the seven-hole probe is clearly sensitive to flow misalignment. Furthermore, the centre port becomes more sensitive to flow misalignment at larger Mach numbers. The centre port of the seven-hole probe returns the correct total pressure within only about $5^{\circ}$ of flow misalignment. The results indicate that the seven-hole probe needs to be calibrated for all angles of misalignment for it to be used to obtain flow pressure and other quantities.

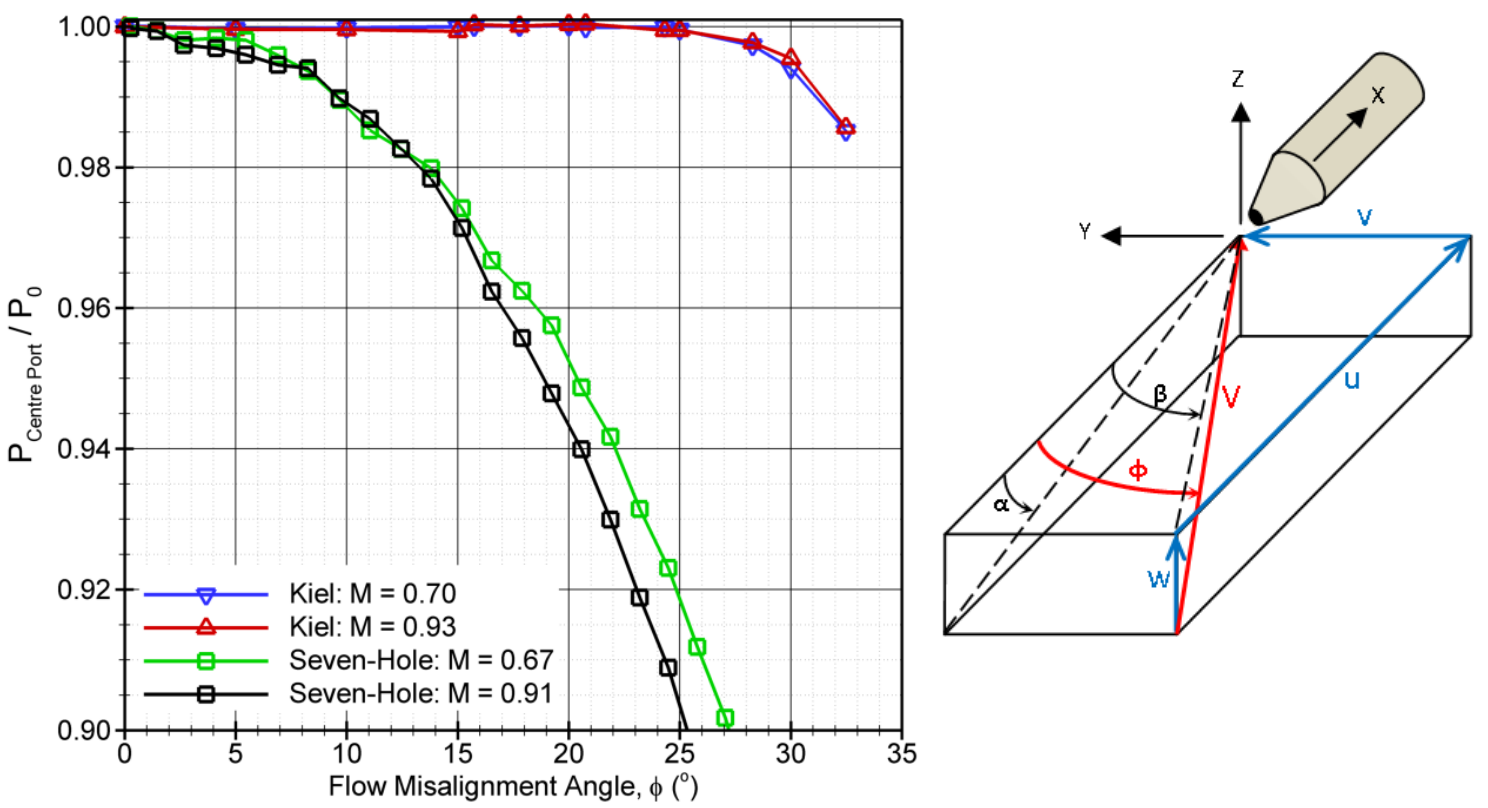

Figure 3.7 Kiel probe and seven-hole probe flow angularity response

\subsection{Seven-Hole Probe Calibration}

The seven-hole probe was calibrated at four different Mach numbers $(0.48<\mathrm{M}<$ $0.94)$ in $2^{\circ}$ increments of pitch $(\alpha)$ and yaw $(\beta)$. Probe calibration was performed for the low flow angle regime $\left(-30^{\circ}<\alpha, \beta<+30^{\circ}\right)$ since larger flow angles were not anticipated for the flow field of interest downstream of the cascade. The minimum Reynolds number based on the probe tip diameter was larger than 15,000 due to the large Mach numbers, 
and therefore, the probe calibration coefficients were expected to be insensitive to Reynolds number variations (Dominy and Hodson, 1993).

The acquired data from the seven-hole probe were analyzed using seven-hole probe calibration tables to extract the flow variables of interest: $P_{0}, P_{S}, \alpha$ and $\beta$. Data reduction was carried out by an iterative solver using table lookup procedures at different Mach numbers. The procedure utilizes a third-order polynomial fit for the coefficient tables for the four different Mach numbers, and linear interpolation within each table. The data reduction software was extensively tested using seven-hole probe test data at various Mach numbers and flow angles. The seven-hole probe calibration coefficients are defined as follows (Johansen et al., 2001):

- Pitch angle coefficient: $\quad C_{\alpha}=\frac{2\left(P_{4}-P_{1}\right)+\left(P_{3}+P_{5}-P_{2}-P_{6}\right)}{3\left(P_{7}-\overline{P_{1-6}}\right)}$

- Yaw angle coefficient: $\quad C_{\beta}=\frac{\left(P_{2}+P_{3}\right)-\left(P_{5}+P_{6}\right)}{\sqrt{3}\left(P_{7}-\overline{P_{1-6}}\right)}$

- Total pressure coefficient: $\quad C_{0}=\frac{P_{7}-P_{0}}{P_{7}-\overline{P_{1-6}}}$

- Dynamic pressure coefficient: $\quad C_{q}=\frac{P_{7}-\overline{P_{1-6}}}{P_{0}-P_{S}}$

Figure 3.8 shows the contour plots of the calibration coefficients at Mach 0.78: $C_{\alpha}$ and $C_{\beta}$ are primarily functions of $\alpha$ and $\beta$, whereas $C_{0}$ and $C_{q}$ correlate well with the resultant flow misalignment angle, $\varphi$. Although not shown here, all four coefficients are also functions of Mach number. $C_{0}$, the correction factor applied to the centre-port pressure of the seven-hole probe as a function of flow misalignment, is used to determine the total pressure (e.g. $C_{0} \approx 0$ for $-5^{\circ}<\varphi<+5^{\circ}$ ). $C_{q}$ shows an asymmetric distribution, which was also observed for a very similar probe at low-speed conditions (Knezevici, 2011), and is subsequently used to determine the static pressure. The resultant flow velocity is determined by combining the Mach numbers and the temperature measurements for every data point. 

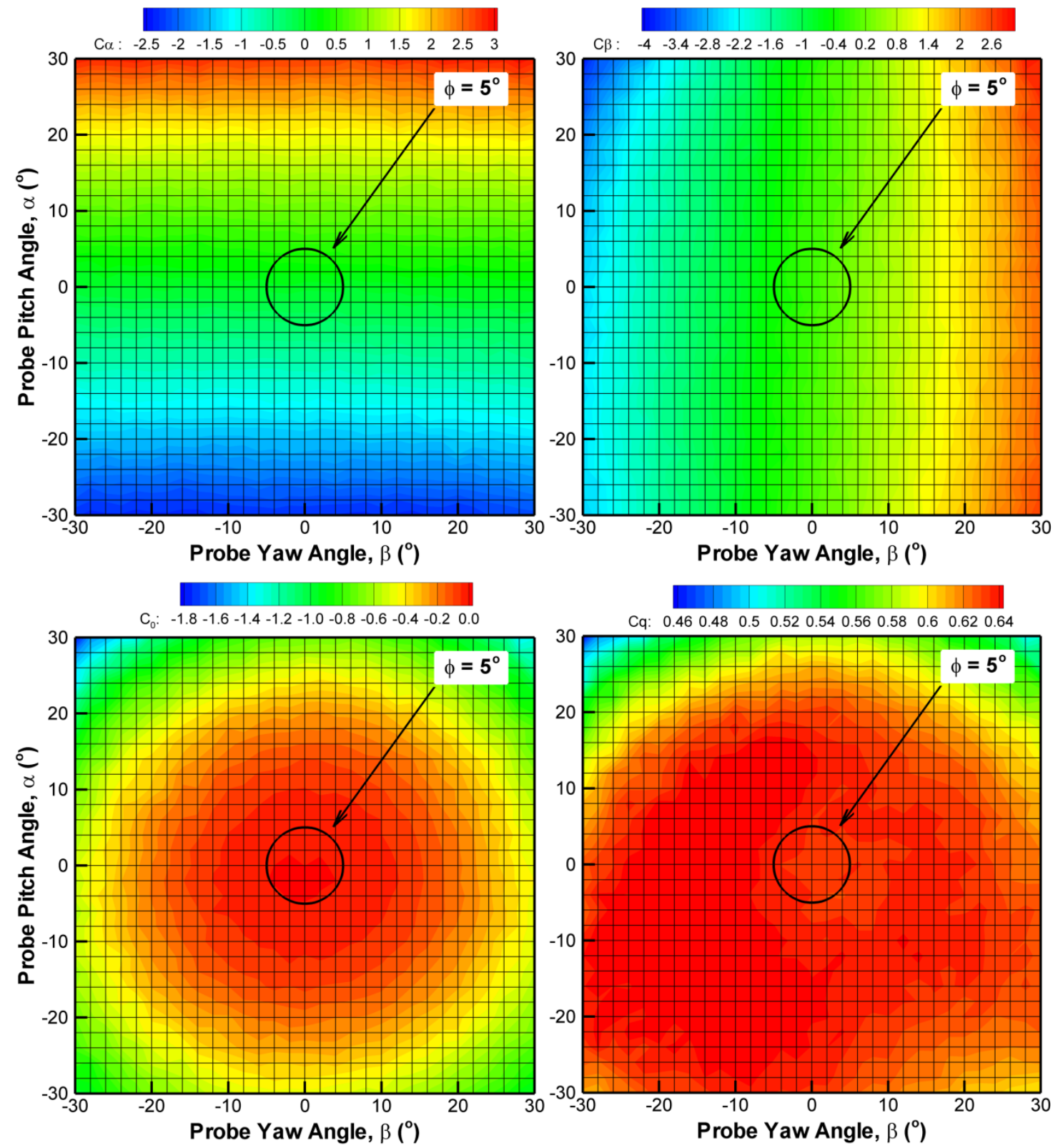

Figure 3.8 Seven-hole probe calibration coefficients (Mach 0.78): $C_{\alpha}$ (top-left), $C_{\beta}$ (topright), $C_{0}$ (bottom-left) and $C_{q}$ (bottom-right)

\subsection{Data Acquisition Procedures}

\subsubsection{Sampling Rates and Times}

The selections of the sampling rates and times have been described by Jeffries (1997). The main objective is to obtain steady-state pressures that are independent of the sampling procedure while minimizing the data collection time due to the limited wind 
tunnel run times (about 40 seconds). A secondary objective is to minimize the effects of small blowing pressure fluctuations on the collected data, which would be present if there is a significant time lag between two subsequent measurements (e.g. port pressures of the seven-hole probe). These objectives were accomplished by collecting 200 pressure samples at each data point at a sampling rate of $2 \mathrm{kHz}$. The step-response of the system was also analyzed by moving the probe directly from the free-stream to the centre of the wake downstream of the cascade, resulting in a total pressure drop, and continuously sampling. Based on these results, a $250 \mathrm{~ms}$ delay was implemented after moving the probe prior to data collection to allow the pressure in the system to reach steady state. Although the data acquisition card is not capable of simultaneous sampling, the relatively high combined sampling rate of $250 \mathrm{kS} / \mathrm{s}$ allows for a total sampling time of 0.1 second to be obtained essentially simultaneously for all eleven channels.

\subsubsection{Inlet and Exit Measurements}

The inlet endwall boundary layers were traversed using a Pitot probe and determined to be fully turbulent in all cases, based on the measured shape factor $(H)$ of 1.4. The inlet endwall boundary layer measurements are presented and discussed in Chapter 7. The free-stream turbulence at the inlet of the test section has been characterized by Corriveau (2005); the inlet free-stream turbulence intensity is about $4 \%$ and the integral length scale is approximately $15 \mathrm{~mm}$, which is about $60 \%$ of axial chord for the cascades used in the present study. These values were determined to be approximately constant over the small range of inlet Mach numbers encountered $\left(0.265<\mathrm{M}_{1}<0.295\right)$ in the present measurements. The degree of anisotropy in inlet turbulence has not been examined.

Pressure probe measurements of the three-dimensional flow field downstream of the cascade were typically collected at 15 spanwise locations starting at 50\% span and moving towards the endwall, with the last location at about 3\% span. At every spanwise location, 41 evenly-spaced pitchwise data points were used to give a reasonable measurement grid of about 600 data points, while keeping the wind tunnel run duration acceptable. A sample of the measurement grid is shown in Figure 3.9.

The wind tunnel run duration decreases as the outlet Mach number is raised. Therefore, a total of two to four runs were required to collect the 41 pitchwise data points 


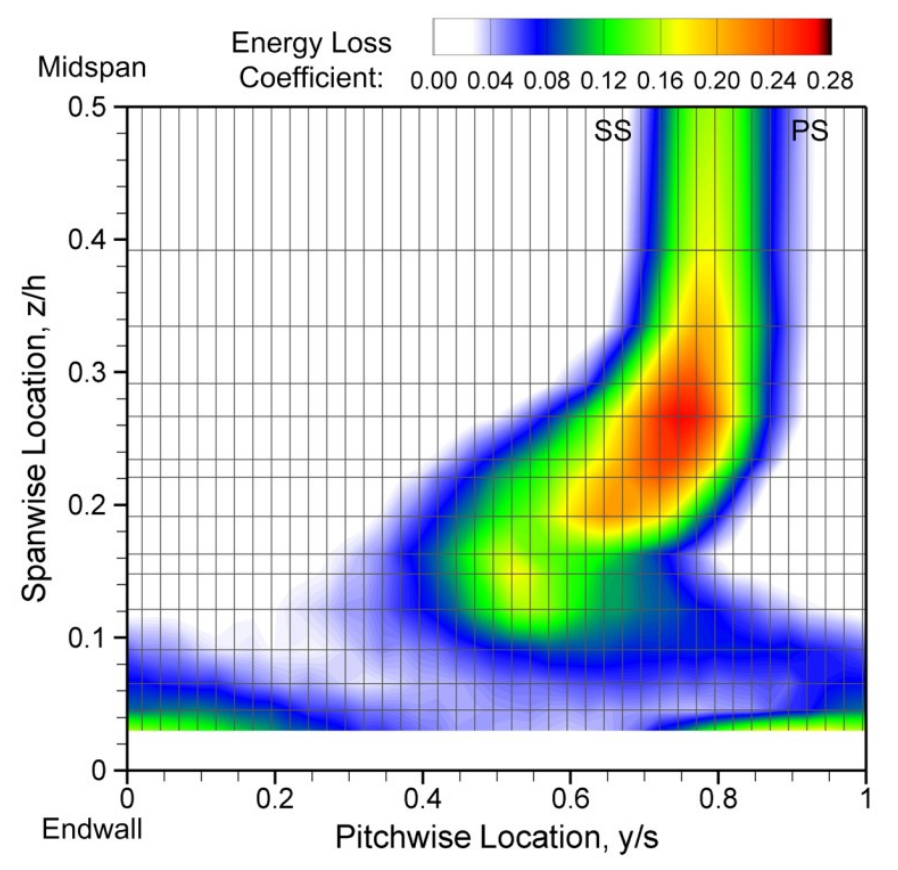

Figure 3.9 Typical measurement grid

at the highest Mach numbers. The collected data was plotted and verified after each traverse to identify any potential outliers; for example, if there were any discrepancies due to spikes in the blowing pressure, the traverse would be repeated. The location of the probe holder, and thus the probe were also manually verified following every traverse.

\subsubsection{Static Pressure Measurements}

Static pressure measurements were collected using static taps at the inlet and exit of the cascade. As shown in Figure 3.2, the inlet static taps are located at 1.5 axial chords upstream of the leading edge extending across all blade passages. The outlet static taps are located in line with the main measurement plane at 1.4 axial chords downstream of the leading edge ( $1.4 \mathrm{C}_{\mathrm{X}}$ measurement plane). Typically, the endwalls were instrumented with four static taps per passage, and each cascade contained six or seven passages. Using a pressure manifold, four adjacent static taps were connected to a transducer to record the endwall static pressure at every data point. In order to document the blade loading distributions, the two airfoils adjacent to the centre passage were also instrumented with midspan surface static taps: five static taps on the pressure surface and eight static taps on the suctions surface. The loading distributions are presented and discussed in Chapter 6. 


\subsection{Surface Flow Visualization Procedures}

The surface flow visualization studies were conducted using a mixture of fluorescentdye and motor oil, illuminated and photographed under ultra-violet lighting following the completion of the tests. The mixture was applied to both the blade surfaces and the endwalls and the tunnel was operated several times prior to disassembly. In addition, videos were recorded during every flow visualization run to ensure that a steady pattern was established prior to the interpretation of the limiting streamlines.

\subsection{Test Section Flow Quality Analysis}

In order to ensure that the cascade accurately models the flows in an infinite row of blades, several conditions need to be fulfilled. In particular, the flow across the inlet of the cascade should be uniform and the downstream flow should be periodic from passage to passage. In the current project, the wind tunnel flow quality was characterized after every new cascade installation. Once the flow quality requirements were verified, the centre blade passage in the cascade was used for collecting all the subsequent measurements.

The inlet static taps, shown in Figure 3.2, were used to examine the inlet flow uniformity in the pitchwise direction. As shown by Corriveau and Sjolander (2002), the deliberate distortion of the inlet flow by misalignment of the inlet side walls may be detected from pitchwise variation in the static pressures, measured on the upstream endwall. The outlet static tap measurements were also recorded to examine the variation in outlet static pressure on the endwall. Figure 3.10 shows an example of the Mach number distributions calculated using inlet centreline total pressure and static tap pressures, identified as PS1 and PS2, for several blowing pressures $\left(1.25<P_{b}<1.30\right)$. The periodic pattern in outlet Mach numbers is associated with the airfoil wakes.

The blade surface static taps were used to assess the blade loading distributions. Figure 3.11 shows an example of the surface isentropic Mach number distributions: the solid line is for the CFD results at the design Mach number and the symbols are for the experimental data at several outlet Mach numbers. The outlet isentropic Mach number was determined as a function of the inlet centerline total pressure, and the outlet static pressure at midspan, area-averaged in the free-stream region between the wakes. 


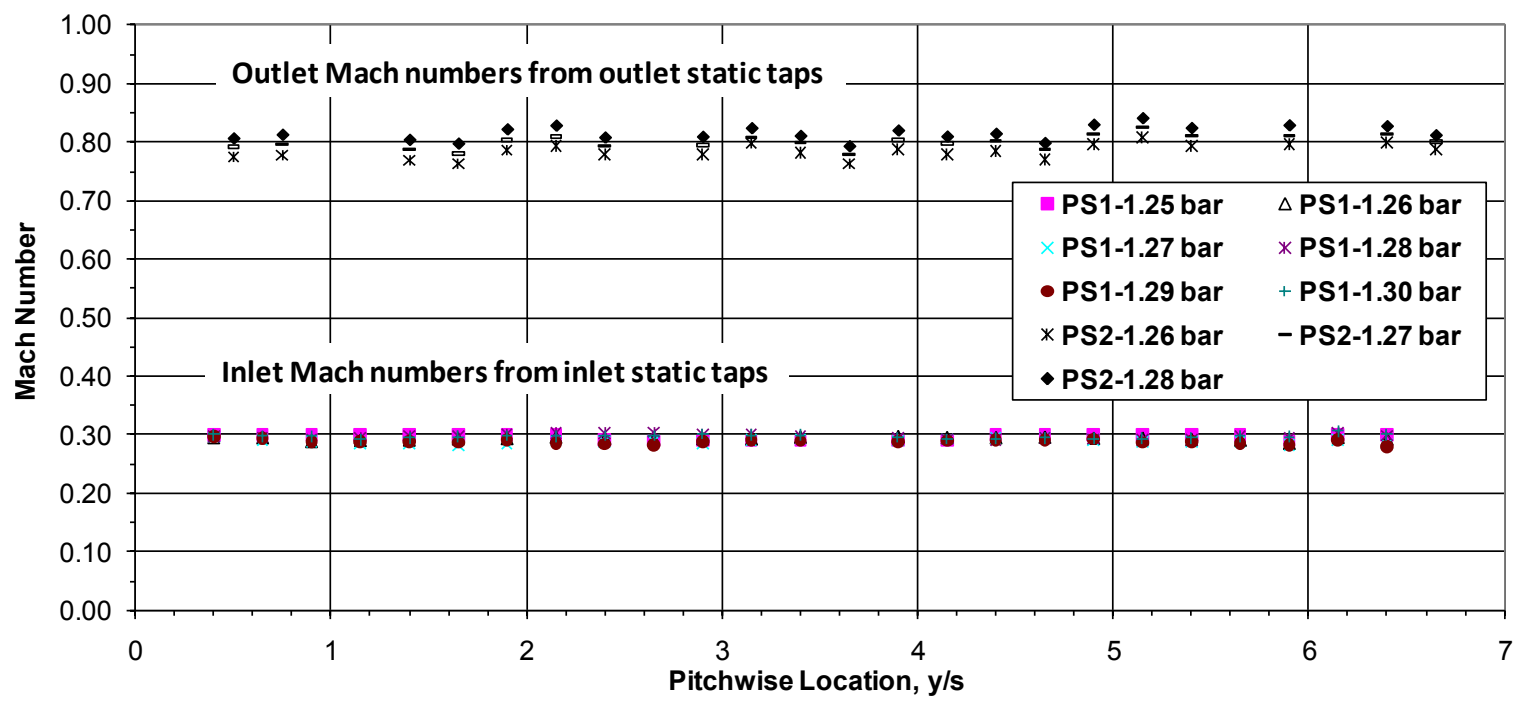

Figure 3.10 Inlet and outlet Mach number distributions based on static tap pressures

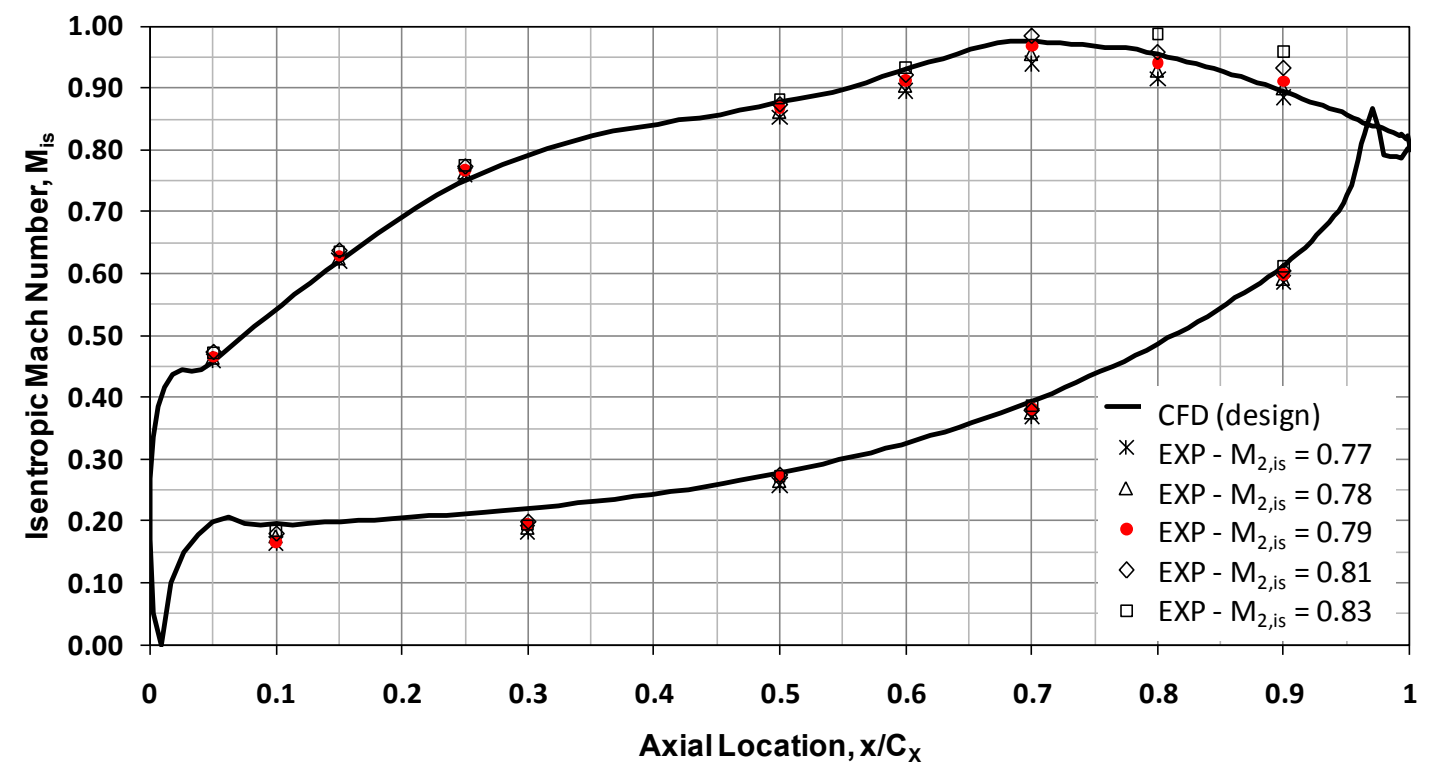

Figure 3.11 Blade surface isentropic Mach number distributions

For a given airfoil geometry and spacing, the loading distribution is primarily influenced by two parameters: inlet incidence and outlet Mach number. The effects of inlet incidence on the loading distribution are mainly apparent on the forward part of the passage, whereas the outlet Mach number influences the suction-surface pressure distribution on the aft part of the airfoil. As shown in Figure 3.11, the wind tunnel blowing pressure was adjusted to match the CFD predictions at the design Mach number; in this case the red-coloured symbols show the best match, particularly on the suction surface. The blade loading distributions are discussed in detail in Chapter 6 . 
The outlet flow periodicity was characterized by traversing and comparing the midspan wakes over the middle five blade passages with the seven-hole probe located at the $1.4 C_{X}$ measurement plane. Figure 3.12 shows an example of the total pressure deficit and the outlet Mach numbers at the design Mach number. As shown, good outlet flow periodicity was achieved without the use of tailboards that are commonly employed in low-speed wind tunnels (e.g. by Knezevici, 2011). The axial velocity density ratio $(A V D R)$ was also calculated.

$$
A V D R=\frac{\int_{0}^{1}\left(\rho_{2} u_{2}\right)_{M S} d(y / s)}{\int_{0}^{1}\left(\rho_{1} u_{1}\right)_{M S} d(y / s)}
$$

A value of 1.0 for the axial velocity density ratio is a necessary, but not sufficient, condition for the presence of two-dimensional blade flow at midspan. In the present experiment, the value of $A V D R$ was found to be $1.0 \pm 0.03$ across the five airfoils, supporting good midspan two-dimensionality.

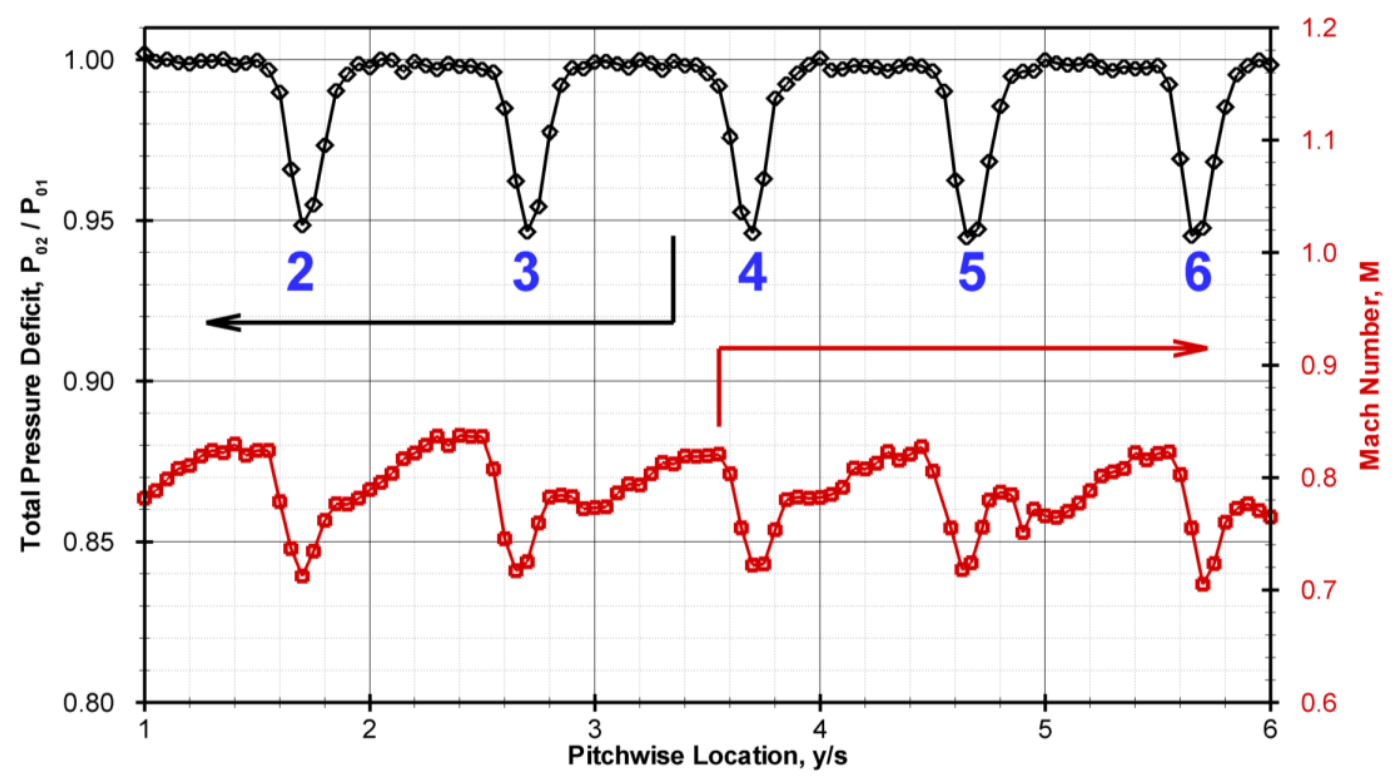

Figure 3.12 Outlet flow periodicity results at the design Mach number

\subsection{Turbine Cascade Geometries}

A total of seven linear turbine cascades, designed and provided by PWA, were examined in the current study. The cascades are based on five distinct turbine airfoil geometries. Two of the airfoil geometries were investigated for both flat and contoured endwalls, as identified in Table 3.2. 
For results, "F" and "C" are appended to the names to indicate the use of flat and contoured endwalls, respectively. Thus, the contoured endwall results for SL2 are designated SL2C, etc. Conventionally, cascade endwalls are machined flat, which approximates the geometry normally used in the engines. The contoured endwalls were designed with the aim of reducing the secondary losses through the turbine cascades. Further details regarding the design and optimization of the contoured endwalls, and the resulting endwall geometries are presented in Chapter 8. The "P" designation refers to the pressure-side modification of the SL2 airfoil, resulting in the SL2P cascade, which was also aimed at reducing the secondary losses without the need for the more costly endwall contouring.

Table 3.2 Turbine cascade configurations

\begin{tabular}{|c|c|c|}
\hline \multirow{2}{*}{ Airfoil Geometry } & \multicolumn{2}{|c|}{ Endwall Configuration } \\
\cline { 2 - 3 } & Flat & Contoured \\
\hline SL1 & X & X \\
\hline SL2 & X & X \\
\hline SL2P & X & - \\
\hline SL3 & X & - \\
\hline SL4 & X & - \\
\hline
\end{tabular}

The main geometric and aerodynamic parameters of the cascades are listed in Table 3.3. The cascade nomenclature is presented in Figure 3.13 and the airfoil geometries are presented in Figure 3.14. Also depicted are the spacings between the airfoils, which influence the overall loading levels.

As indicated in Table 3.3, the cascades are designed with the same airfoil span, axial chord length and for the same design outlet Mach number. There are differences in pitch, true chord and total flow turning, however, which result in different Zweifel coefficients. The cascades may be grouped either by total flow turning or Zweifel coefficient. Categorized by flow turning, SL3 and SL4 are considered low flow-turning, whereas SL1, SL2 and SL2P are considered high flow-turning. For the same level of loading, high-turning cascades typically generate larger secondary losses than low-turning cascades (Yamamoto, 1987a; Yamamoto, 1987b). In terms of Zweifel coefficient, SL1 and SL3 are considered baseline loaded, whereas SL2, SL2P and SL4 are considered 
more highly loaded. Additional details regarding the cascade geometries are presented in Chapters 6 through 9.

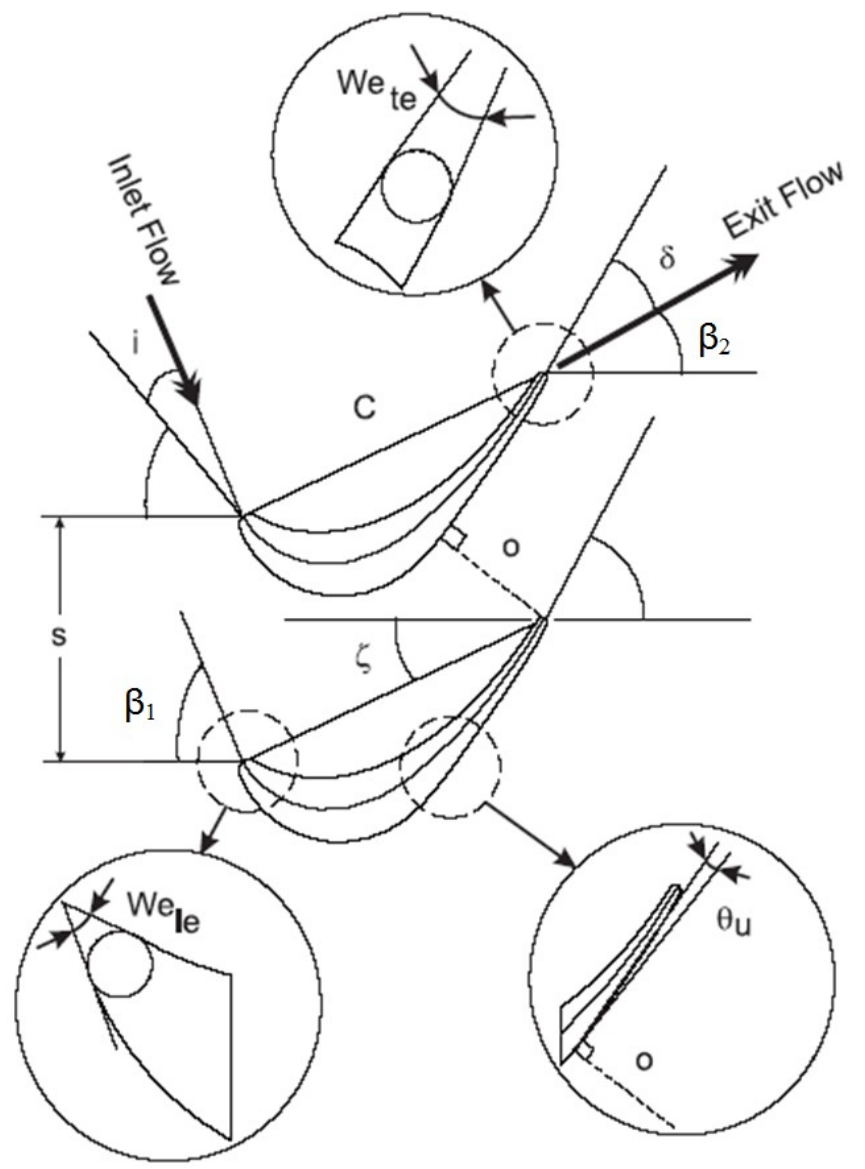

Figure 3.13 Turbine cascade nomenclature (reproduced from Corriveau, 2005)

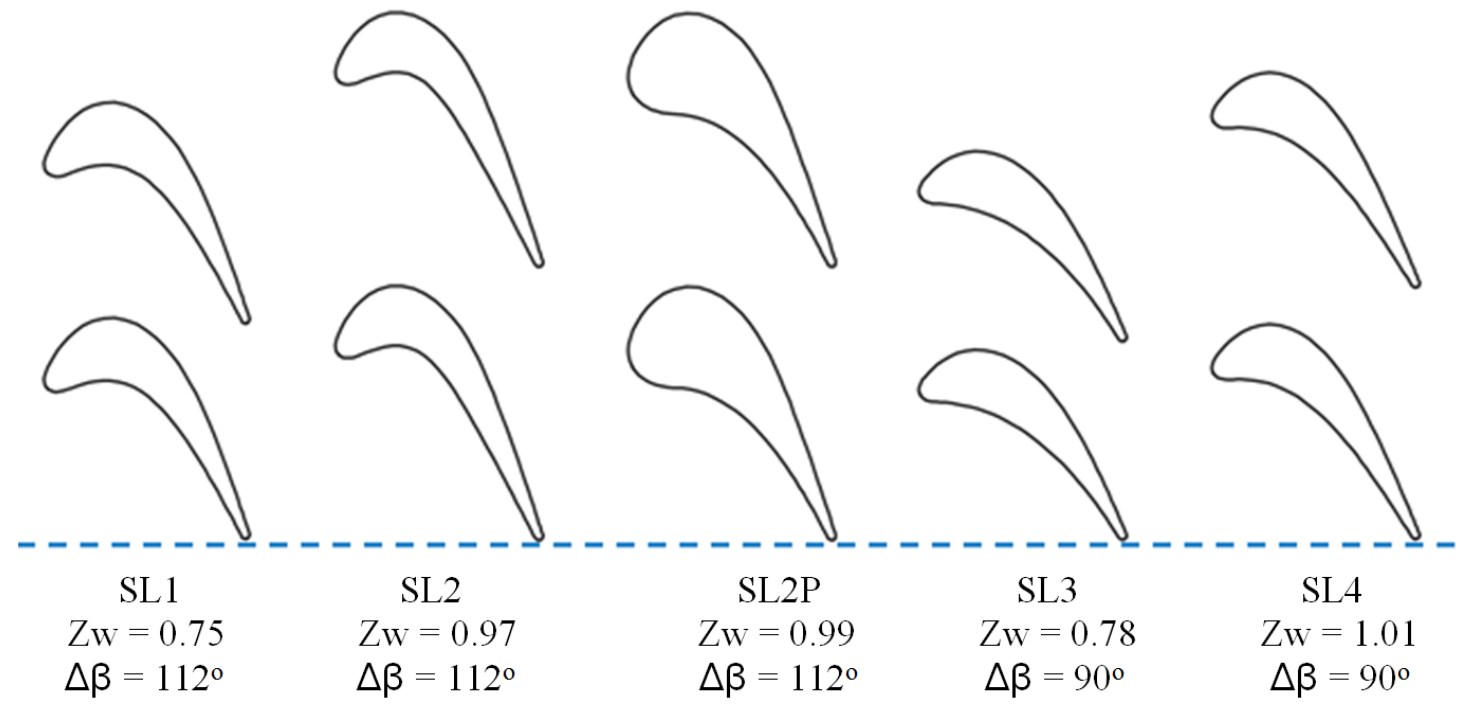

Figure 3.14 Turbine airfoil geometries 
Table 3.3 Turbine cascade parameters

\begin{tabular}{|c|c|c|c|c|c|}
\hline & SL1F & SL2F & SL2P & SL3F & SL4F \\
\hline \multicolumn{6}{|l|}{ Geometric Parameters } \\
\hline Blade Span, h (mm) & 61.0 & 61.0 & 61.0 & 61.0 & 61.0 \\
\hline Axial Chord, $C_{X}(\mathrm{~mm})$ & 25.4 & 25.4 & 25.4 & 25.4 & 25.4 \\
\hline True Chord, C (mm) & 31.0 & 33.7 & 33.7 & 30.8 & 32.4 \\
\hline Pitch, s (mm) & 26.8 & 34.0 & 34.0 & 24.6 & 31.4 \\
\hline Solidity, C/s & 1.16 & 0.99 & 0.99 & 1.25 & 1.03 \\
\hline Uncovered Turning, $\theta_{U}\left({ }^{\circ}\right)$ & 16.3 & 19.2 & 19.2 & 17.0 & 19.3 \\
\hline Stagger Angle, $\zeta\left(^{\circ}\right)$ & 34.9 & 41.2 & 41.2 & 34.5 & 38.3 \\
\hline LE Wedge Angle, $W_{\text {le }}\left(^{\circ}\right)$ & 40.0 & 44.0 & 95.0 & 52.0 & 50.0 \\
\hline TE Wedge Angle, $W_{\text {te }}\left({ }^{\circ}\right)$ & 9.0 & 9.0 & 9.0 & 9.0 & 9.0 \\
\hline Max Thickness, $\mathbf{t}_{\mathbf{m}}(\mathbf{m m})$ & 7.9 & 7.5 & 13.1 & 7.4 & 7.4 \\
\hline Throat Opening, o (mm) & 9.5 & 12.0 & 12.0 & 10.7 & 13.7 \\
\hline \multicolumn{6}{|l|}{ Aerodynamic Parameters } \\
\hline Inlet Flow Angle, $\beta_{1}\left({ }^{\circ}\right)$ & 43.5 & 43.5 & 43.5 & 27.0 & 27.0 \\
\hline Outlet Flow Angle, $\beta_{2}\left({ }^{\circ}\right)$ & 69.1 & 68.2 & 68.5 & 62.9 & 63.0 \\
\hline Total Flow Turning, $\Delta \beta\left({ }^{\circ}\right)$ & 112.6 & 111.7 & 112.0 & 89.9 & 90.0 \\
\hline Nominal Inlet Mach Number, $M_{1}$ & 0.286 & 0.283 & 0.294 & 0.305 & 0.302 \\
\hline Nominal Outlet Isentropic Mach Number, $M_{2, \text { is }}$ & 0.78 & 0.78 & 0.78 & 0.78 & 0.78 \\
\hline Inlet Reynolds Number, $\operatorname{Re}_{\mathrm{C}, 1}$ & 270,000 & 280,000 & 280,000 & 290,000 & 300,000 \\
\hline Outlet Reynolds Number, $\operatorname{Re}_{\mathrm{C}, 2}$ & 580,000 & 600,000 & 590,000 & 580,000 & 620,000 \\
\hline Zweifel Coefficient, Zw & 0.75 & 0.97 & 0.99 & 0.78 & 1.01 \\
\hline
\end{tabular}

\subsection{Test Matrices}

The test matrices for the low-turning and the high-turning cascades are presented in Table 3.4 and Table 3.5, respectively. All measurements were collected at the design incidence. The outlet Mach numbers, however, were varied for selected cases to examine the effects of flow compressibility on the profile and secondary losses.

The measurements include the aforementioned flow quality checks, seven-hole probe data at all indicated Mach numbers and Kiel probe data at the design Mach number. Initially, the results from the Kiel probe and the seven-hole probe showed small differences within the secondary flow regions. Following modification to the traverse gear and the wind tunnel test section, including improved sealing, the Kiel probe and the seven-hole probe produced very similar results. The data reduction procedures are presented in the next chapter. 
Table 3.4 Test cases for the low-turning cascades: SL3 and SL4 (design Mach number)

\begin{tabular}{|c|c|c|c|c|}
\hline & \multicolumn{3}{|c|}{ SL3F } & SL4F \\
\hline Measurement Location & $1.4 \mathrm{C}_{X}$ & $1.7 \mathrm{C}_{X}$ & $2.0 \mathrm{C}_{X}$ & $1.4 \mathrm{C}_{X}$ \\
\hline Outlet Mach Number & 0.785 & 0.788 & 0.789 & 0.779 \\
\hline
\end{tabular}

Table 3.5 Test cases for the high-turning cascades: SL1, SL2 and SL2P (design and offdesign Mach numbers)

\begin{tabular}{|c|c|c|c|c|c|c|c|c|}
\hline & \multicolumn{4}{|c|}{ SL1F } & \multicolumn{4}{c|}{ SL1C } \\
\hline Measurement Location & $1.4 \mathrm{C}_{X}$ & $1.4 \mathrm{C}_{X}$ & $1.4 \mathrm{C}_{X}$ & $2.0 \mathrm{C}_{X}$ & $1.4 \mathrm{C}_{X}$ & $1.4 \mathrm{C}_{X}$ & $1.4 \mathrm{C}_{X}$ & $2.0 \mathrm{C}_{X}$ \\
\hline Outlet Mach Number & 0.651 & 0.781 & 0.910 & 0.783 & 0.649 & 0.782 & 0.910 & 0.779 \\
\hline
\end{tabular}

\begin{tabular}{|c|c|c|c|c|c|c|c|c|}
\hline & \multicolumn{4}{|c|}{ SL2F } & \multicolumn{4}{c|}{ SL2C } \\
\hline Measurement Location & $1.4 \mathrm{C}_{X}$ & $1.4 \mathrm{C}_{\mathrm{X}}$ & $1.4 \mathrm{C}_{\mathrm{X}}$ & $2.0 \mathrm{C}_{\mathrm{X}}$ & $1.4 \mathrm{C}_{\mathrm{X}}$ & $1.4 \mathrm{C}_{\mathrm{X}}$ & $1.4 \mathrm{C}_{\mathrm{X}}$ & $2.0 \mathrm{C}_{\mathrm{X}}$ \\
\hline Outlet Mach Number & 0.652 & 0.775 & 0.908 & 0.777 & 0.650 & 0.773 & 0.905 & 0.779 \\
\hline
\end{tabular}

\begin{tabular}{|r|c|c|c|c|c|c|c|c|c|}
\hline & \multicolumn{10}{|c|}{ SL2PF } \\
\hline Measurement Location & $1.4 \mathrm{C}_{X}$ & $1.4 \mathrm{C}_{X}$ & $1.4 \mathrm{C}_{X}$ & $1.7 \mathrm{C}_{X}$ & $1.7 \mathrm{C}_{X}$ & $1.7 \mathrm{C}_{X}$ & $2.0 \mathrm{C}_{X}$ & $2.0 \mathrm{C}_{X}$ & $2.0 \mathrm{C}_{X}$ \\
\hline Outlet Mach Number & 0.645 & 0.778 & 0.898 & 0.647 & 0.777 & 0.896 & 0.646 & 0.779 & 0.897 \\
\hline
\end{tabular}




\section{Chapter 4}

\section{Data Reduction Procedures and Uncertainty Analysis}

\subsection{Introduction}

The main objective of the experimental investigations was to determine the entropy generation within the blade rows based on the total pressure measurements. Additional parameters such as exit flow angles, secondary kinetic energy and vorticity were extracted from the data to get a better understanding of the loss generating mechanisms. The significance of these parameters was described in Chapter 2; their definitions are presented here.

The data obtained from the seven-hole pressure probe are processed and presented in two ways: as contour plots and averaged values at the measurement plane (based on the integral system effects approach), and in terms the fully mixed-out results (based on the complete equilibrium approach). The loss coefficients, secondary flow parameters and the averaging techniques are described in section 4.2. The experimental uncertainties are summarized in section 4.3 .

\subsection{Loss Coefficients and Secondary Flow Parameters}

\subsubsection{Entropy Production and Loss Coefficients}

For an ideal gas, the entropy generation within the blade row may be estimated from:

$$
s_{2}-s_{1}=c_{p} \ln \left(\frac{T_{02}}{T_{01}}\right)-R \ln \left(\frac{P_{02}}{P_{01}}\right)
$$

Since there is no work exchange in a linear turbine cascade and the flow is assumed to be expanded adiabatically, the stagnation temperature remains constant between the 
inlet and outlet planes. Therefore, the entropy generation can be directly related to the changes in stagnation pressure:

$$
\Delta s=-R \ln \left(\frac{P_{02}}{P_{01}}\right)=R \ln \left(\frac{P_{01}}{P_{02}}\right)
$$

For small changes in total pressure, the entropy generation may be expressed as:

$$
\Delta s=R \frac{\left(P_{01}-P_{02}\right)}{P_{01}}
$$

Various definitions of loss coefficients have been proposed based on this relationship. If the outlet static pressure is not available (e.g. for Kiel probe data), the total pressure deficit may be expressed as:

$$
\Delta P_{0}=\frac{P_{01}-P_{02}}{P_{01}}
$$

In the present study, the outlet static pressure is available from the seven-hole probe measurements. The total pressure loss coefficient for compressible flows is defined as:

$$
Y=\frac{P_{01}-P_{02}}{P_{02}-P_{2}}=\frac{\frac{P_{01}}{P_{02}}-1}{1-\frac{P_{2}}{P_{02}}}=\frac{\frac{P_{01}}{P_{02}}-1}{1-\left(1+\frac{\gamma-1}{2} M_{2}^{2}\right)^{\frac{-\gamma}{\gamma-1}}}
$$

Alternative loss coefficients may also be derived based on the differences in enthalpy between the inlet and outlet stations (Brown, 1972; Perdichizzi, 1990; Mee et al., 1992). The enthalpy-entropy diagram for the expansion process through a turbine is shown in Figure 4.1.

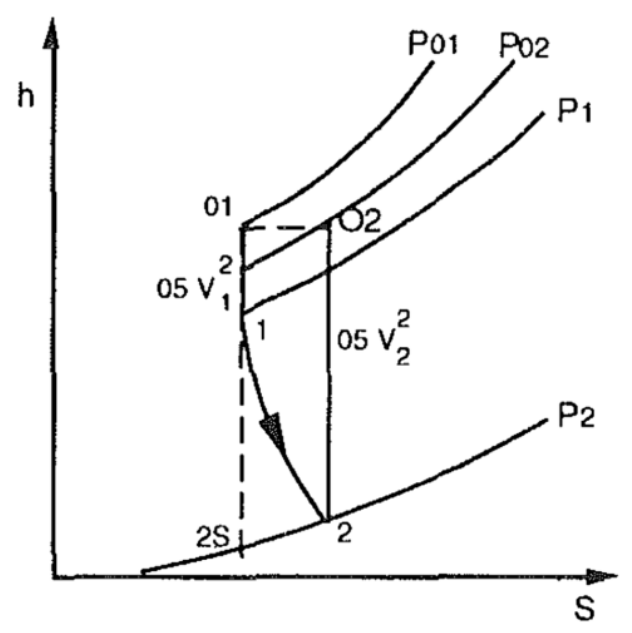

Figure 4.1 Enthalpy-entropy diagram for turbine (reproduced from Denton, 1993) 
Assuming a constant slope for the isobar between stations " 2 " and " $2 \mathrm{~S}$ " for small changes in stagnation pressure, the energy loss coefficient may be defined as:

$$
\zeta=\frac{T_{0} \Delta s}{h_{0}-h_{2 S}}=\frac{T_{0} R \ln \left(\frac{P_{01}}{P_{02}}\right)}{h_{0}-h_{2 S}}=\frac{T_{0} R \ln \left(\frac{P_{01}}{P_{02}}\right)}{c_{p}\left(T_{0}-T_{2 S}\right)}=\frac{\left(\frac{\gamma-1}{\gamma}\right) \ln \left(\frac{P_{01}}{P_{02}}\right)}{1-\left(1+\frac{\gamma-1}{2} M_{2, i s}^{2}\right)^{-1}}
$$

The numerator in Equation 4.6 is defined as the product of entropy generation and total temperature $\left(T_{0} \Delta S\right)$. The total temperature is selected here since it remains constant between the inlet and outlet planes in the linear turbine cascade. The denominator $\left(h_{0}-\right.$ $h_{2 S}$ ) represents the available (remaining) kinetic energy per unit mass for a reversible process. In other words, the energy loss coefficient is defined as the lost ability to perform work as a fraction of outlet (isentropic) kinetic energy. The energy loss coefficient has been recommended by Brown (1972) for transonic turbine data reduction as it shows the least sensitivity to small changes in Mach number (although not independent), for instance due to small fluctuations in wind tunnel blowing pressure. The results for both the total pressure loss coefficient and the energy loss coefficient are presented in the following chapters. The results from several alternative definitions of energy loss coefficient were also examined, but are not presented here since they showed very similar trends to the coefficients examined here. It should be noted that the results from various definitions of loss coefficients may be derived from each other with the knowledge of the outlet Mach number.

In the absence of tip gap flows, the overall total pressure losses are divided into profile and secondary losses. Profile losses are defined as the sum of blade surface and trailing edge losses in this case. Results from two different loss breakdown schemes are presented in the following chapters: the classical method where profile losses are assumed constant along the span, and the more recent method proposed by Benner et al. (2006a) based on the penetration depth of the passage vortex separation line. Both the classical loss breakdown scheme and the secondary flow penetration-based method define the total loss coefficient as the summation of profile and secondary losses:

$$
Y_{T}=Y_{P}+Y_{S}
$$

However, Benner et al.'s method defines the profile loss coefficient based on the midspan losses and the penetration depth of the secondary losses as shown in Figure 4.2. The new 
profile loss coefficient definition, presented in Equation 4.8, is particularly relevant for turbine cascade airfoils with lower aspect ratios. Furthermore, it allows for the assessment of secondary losses for stalled airfoils, which typically exhibit rather large midspan losses (possibly resulting in negative secondary losses based on the classical loss breakdown method).

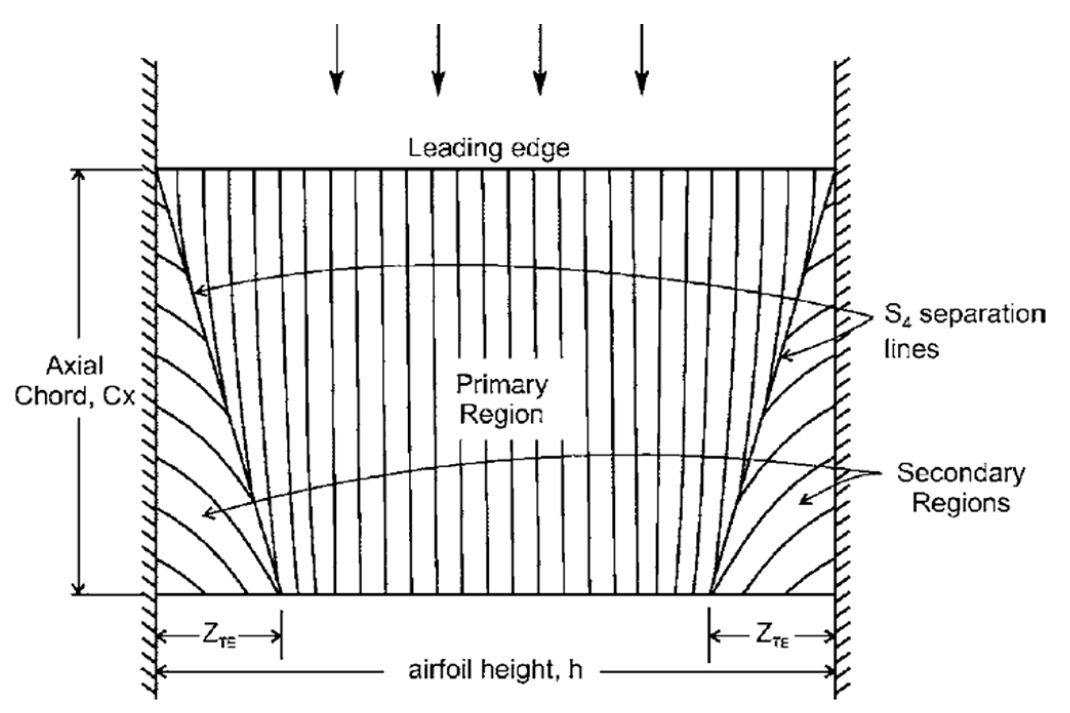

Figure 4.2 Suction surface definition for the new loss breakdown scheme (reproduced from Benner et al., 2006a)

$$
Y_{P}=Y_{\text {midspan }}\left(\frac{A_{\text {primary }}}{A_{\text {secondary }}}\right)=Y_{\text {midspan }}\left(1-\frac{Z_{T E}}{h}\right)
$$

\subsubsection{Other Parameters of Interest}

The flow acceleration through the turbine passage is quantified using three different parameters: convergence ratio $(C R)$, which is typically used in low-speed facilities, Mach number ratio $(M R)$ and row velocity ratio $(R V R)$ :

$$
C R=\frac{\cos \beta_{1}}{\cos \beta_{2}} \quad ; \quad M R=\frac{M_{2}}{M_{1}} \quad ; \quad R V R=\frac{V_{2}}{V_{1}}
$$

As indicated in Chapter 3, the axial velocity density ratio is used to assess the two dimensionality of the flow at the midspan of the cascade. The axial velocity density ratio and the axial velocity ratio, which is used in low-speed facilities, are defined as:

$$
A V D R=\frac{\int_{0}^{1}\left(\rho_{2} u_{2}\right)_{M S} d(y / s)}{\int_{0}^{1}\left(\rho_{1} u_{1}\right)_{M S} d(y / s)}
$$




$$
A V R=\frac{\int_{0}^{1}\left(u_{2}\right)_{M S} d(y / s)}{\int_{0}^{1}\left(u_{1}\right)_{M S} d(y / s)}
$$

As shown in Figure 4.3, the compressible form of the Zweifel coefficient is used to characterize the overall blade loading level at midspan.
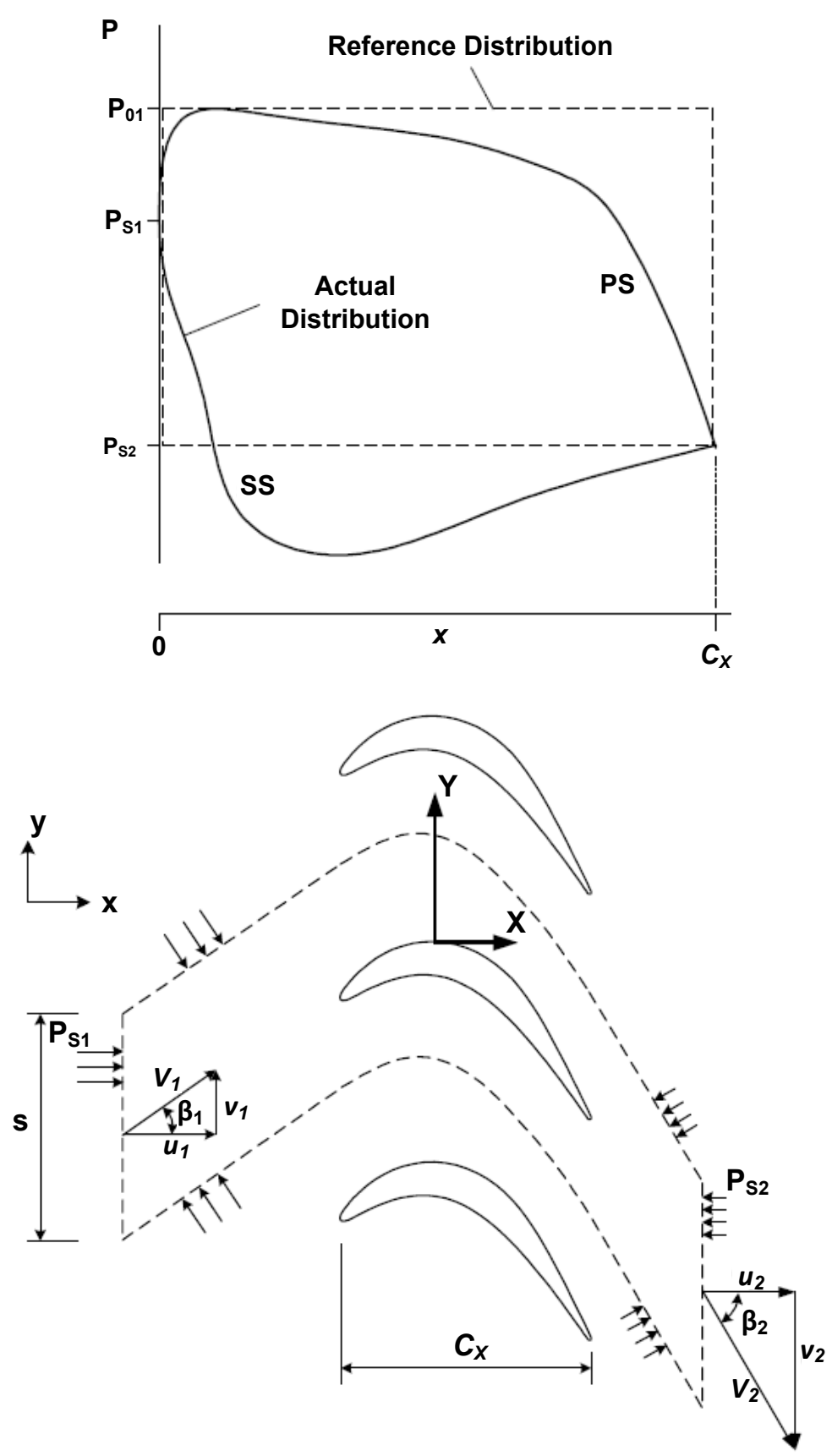

Figure 4.3 Turbine blade loading distribution, and inlet and outlet flow angles (reproduced from Sjolander, 2006) 


$$
\begin{gathered}
Z w=\frac{\text { Actual Loading }}{\text { Reference Loading }}=\frac{\dot{m}_{\text {unit span }}\left(V_{2} \sin \beta_{2}+V_{1} \sin \beta_{1}\right)}{\left(P_{0}-P_{2}\right) C_{X}} \\
Z w=\frac{s}{C_{X}} \frac{\gamma M_{2}^{2} \cos \beta_{2}}{\left(1+\frac{\gamma-1}{2} M_{2, i s}^{2}\right)^{\frac{\gamma-1}{\gamma}}\left(\sin \beta_{2}+\frac{1}{R V R} \sin \beta_{1}\right)}
\end{gathered}
$$

The incompressible form of the Zweifel coefficient, which is typically used for low-speed cascades, is also presented here for comparison purposes:

$$
Z w_{\text {Incompressible }}=2 \frac{s}{C_{X}} \cos ^{2} \beta_{2}\left(\tan \beta_{2}+\frac{1}{A V R} \tan \beta_{1}\right)
$$

The blade surface isentropic Mach number distribution is calculated based on the inlet centerline total pressure and the airfoil surface static pressures:

$$
M_{i s}=\sqrt{\frac{2}{\gamma-1}\left[\left(\frac{P_{01}}{P_{S}}\right)^{\frac{\gamma-1}{\gamma}}-1\right]}
$$

The surface pressure gradients are also analyzed to investigate the possibilities of flow relaminarization under favourable pressure gradients and flow separation under adverse pressure gradients. The non-dimensional pressure gradients are presented in the form of the acceleration parameter (Hourmouziadis, 1989):

$$
A C=\frac{v}{V^{2}} \frac{d V}{d S}
$$

where $v$ and $S$ denote kinematic viscosity and blade surface position, respectively. The flow velocity at the edge of the boundary layer, $V$, is determined as a function of the blade surface isentropic Mach number and the inlet total temperature.

The surface skin friction coefficients were extracted from the CFD results to identify any potential boundary layer separation as indicated by wall shear stress $\left(\tau_{w}=0\right)$ :

$$
C_{f}=\frac{\tau_{w}}{P_{02}-P_{S 2}}
$$

Several additional parameters are investigated to compare the strengths of the secondary flow fields downstream of the cascades; these include exit flow angles $(\Delta \beta)$, streamwise vorticity $\left(\omega_{S}\right)$, enstrophy $(\varepsilon)$ and secondary kinetic energy $(S K E)$. The corresponding coefficients are non-dimensionalized using the outlet isentropic velocity, associated with the core, inviscid flow region downstream of the cascade. The secondary 
flow parameters are derived from the velocity components normal to the primary flow direction. The midspan $(M S)$ primary flow direction, shown in Figure 4.3, is defined as:

$$
\beta_{M S}=\tan ^{-1}\left(\frac{v_{M S}}{u_{M S}}\right)
$$

The components of the vorticity vector are estimated using the method of Perdichizzi (1990) based on the Crocco's relation (see Appendix A for derivation):

$$
\begin{gathered}
\omega_{X}=\frac{\partial w}{\partial y}-\frac{\partial v}{\partial z} \\
\omega_{Y}=\frac{1}{u}\left(v \omega_{X}+\frac{a^{2}}{\gamma} \frac{\partial \ln P_{0}}{\partial z}\right) \\
\omega_{Z}=\frac{1}{u}\left(w \omega_{X}-\frac{a^{2}}{\gamma} \frac{\partial \ln P_{0}}{\partial y}\right)
\end{gathered}
$$

The streamwise vorticity coefficient is defined as the vorticity about the axis that defines the primary flow direction $\left(\beta_{M S}\right)$ :

$$
C_{\omega_{S}}=\frac{C}{V_{2, i s}}\left(\omega_{X} \cos \beta_{M S}+\omega_{Y} \sin \beta_{M S}\right)
$$

The helicity coefficient was also investigated (though not presented here) and found to show similar trends to those of the streamwise vorticity coefficient. The flow velocity components in the plane normal to the primary flow direction are referred to as secondary velocities. The secondary velocity vectors and the secondary kinetic energy coefficient are obtained using:

$$
\begin{gathered}
v_{s e c}=v \cos \beta_{M S}-u \sin \beta_{M S} \quad \text { and } \quad w_{s e c}=w \\
C_{S K E}=\frac{\rho\left(v_{s e c}^{2}+w_{s e c}^{2}\right)}{\gamma P_{2, M S} M_{2, i s}^{2}}
\end{gathered}
$$

The enstrophy coefficient (Davidson, 2005) is used to quantitatively compare the vorticity distributions. Enstrophy is related to the kinetic energy of the vortical structures in the flow field. However, unlike secondary kinetic energy, which is based on the secondary velocity vectors, enstrophy is representative of the energy dissipation due to velocity gradients. Enstrophy, like mechanical energy, is destroyed by viscous forces. The enstrophy coefficient is defined as:

$$
C_{\varepsilon}=\frac{C^{2}}{V_{2, i s}^{2}}\left(\omega_{X}^{2}+\omega_{Y}^{2}+\omega_{Z}^{2}\right)
$$




\subsubsection{Reduction of the Data from the Measurement Plane}

As discussed by Pianko and Vazelt (1982) and Cumpsty and Horlock (2006), there are several methods for averaging the parameters obtained from pressure probes, such as the seven-hole probe used in the present work. The results from the appropriate averaging method should ideally satisfy the equations of motion: mass, momentum and energy. For instance, the total temperature is typically mass averaged to conserve the total enthalpy (although the total temperature distribution is assumed constant here since the distribution was not measured). The static pressure distribution is area averaged to satisfy the momentum equation in the axial direction:

$$
P_{\text {area-ave }}=\frac{1}{A} \int_{0}^{h} \int_{0}^{s} P d y d z
$$

On the other hand, the averaging method for other parameters, such as total pressure and velocity, is not as straightforward with reference to the conservation equations. For instance, the area-averaged axial velocity satisfies the conservation of mass, whereas the mass-averaged velocities satisfy the momentum equation.

The total pressure measurements are mass averaged to account for the effects of flow non-uniformity in the measurement plane:

$$
P_{0_{\text {mass-ave }}}=\frac{1}{\dot{m}} \int_{0}^{h} \int_{0}^{s}(\rho u) P_{0} d y d z
$$

The inlet mass flux variations, across the inlet boundary layer, are determined using the total pressure from the Pitot probe, the static pressure from the endwall static taps (assuming constant static pressure across the span), and the free-stream total temperature. Axial mass flux distributions downstream of the cascade are determined from the sevenhole probe results. The corresponding plane-averaged Mach number is determined from the mass-averaged total pressure and area-averaged static pressure.

As discussed in Chapter 3, the measurement plane extends from midspan to approximately 3\% span. There were no measurements collected from 0 to $3 \%$ span due to probe/wall interference effects. The contribution of the near-wall region is therefore estimated based on the collected data at 3\% span and near-wall trends obtained from CFD. The near-wall region primarily comprises low momentum fluid with relatively low mass fluxes, which attenuate its contribution to the overall mass-averaged results. Based 
on the CFD predictions, the largest gradients are confined to within $1 \%$ span downstream of the cascade. Furthermore, the flow angles and static pressure distributions remain nearly uniform between 0 and $3 \%$ span. Therefore, a phantom row of nodes is added at $1 \%$ span based on the data from the seven-hole probe:

- The total pressure at $1 \%$ span was extrapolated from the near wall traverses (e.g. $3 \%$ and $4 \%$ span measurements).

- The static pressure and flow angles at $1 \%$ span were taken to be equal to the measured results at $3 \%$ span.

The pitchwise distributions of the parameters at the endwall are obtained in a similar fashion:

- The limiting total pressure at the endwall was taken to be equal to the wall static pressure as estimated from the static pressure measured at 3\% span.

- Other parameters, such as velocities, were specified based on the no slip condition at the endwall.

The plane-averaged results are calculated based on the cell-centered values. Therefore, the contributions of the limiting total pressures at the endwall are also captured.

The integrated results are also presented in terms of pitchwise-averaged plots to examine and compare the spanwise distributions. The presentation of the pitchwiseaveraged plots not only includes the effects of mass flux variations across the pitch, but also incorporates the variations across the span; the methodology is presented in Appendix B.

\subsubsection{Loss Mixing-Out Procedure}

Additional losses are generated downstream of the measurement plane due to the mixing out of the secondary flows, and the dissipation of the associated kinetic energy. A control volume analysis is performed to account for this additional loss generation. The control volume extends from the measurement plane, which is non uniform (e.g. total pressure variation), to a hypothetical plane far downstream, where the flow field is completely uniform (Mahallati, 2003). The mixed-out parameters are particularly useful for comparing the results that are collected at different measurement planes (e.g. from different facilities). 
The flow mixing out procedure developed by Oldfield et al. (1981) and Amecke and Safarik (1995) is used to determine the mixed-out values of total and static pressures and flow angles. The loss mixing-out method is based on the conservations of mass and momentum and is applicable to the three-dimensional, compressible flow field downstream of the cascade. The conservation of energy equation is not required here since the total temperature variation downstream of the cascade is assumed negligible $\left(\mathrm{T}_{0}\right.$ is constant). The method also assumes frictionless parallel walls, and therefore does not account for the additional endwall loss generation downstream of the measurement plane.

The mixed-out loss coefficients comprise of the losses at the measurement plane and the additional mixing losses downstream of the measurement plane. The mixing losses are divided into two categories (Benner et al., 2004) as described in Chapter 7:

- Diffusion loss = primary kinetic energy dissipation - static pressure recovery

- Secondary kinetic energy dissipation

The contributions of these two sources are compared in Chapters 6 through 9. The loss mixing-out procedure is described in more detail in Appendix C.

\subsection{Experimental Uncertainties}

\subsubsection{Introduction}

The High-Speed Wind Tunnel Laboratory at Carleton University is a well-established facility, which has been used extensively for similar measurements since the mid-1990s. Therefore, the uncertainty analyses conducted by previous researchers (Islam, 1999; Jeffries, 2000; Jouini, 2000; Corriveau, 2005) are largely applicable to the present results. However, these are the first fully three-dimensional measurements made, using a new probe, the seven-hole pressure probe. In previous experiments, which were focused on profile flows, the static pressure was obtained using a static probe at midspan. During the preliminary phase of the current work, the static pressures at midspan were also recorded using the static probe and a three-hole probe to compare with the seven-hole probe results. However, the static probe was not used at other spanwise locations due to the larger flow misalignment angles in the secondary flow regions. Instead, the static pressure was obtained from the seven-hole probe for all the test cases. Therefore, the

experimental uncertainty estimates are restablished here based on the uncertainty analysis 
and repeatability studies. In particular, the uncertainties associated with the measurements made using the new probe are documented here.

\subsubsection{Sources of Uncertainty and Analysis Procedure}

The uncertainty analysis is based on the method of Mofatt (1982) and Baines et al. (1990). The derived parameters of interest, such as the total pressure loss coefficient, may be expressed in terms of the fundamental parameters, which are assumed to be independent and normally distributed:

$$
F=F\left(\varphi_{1}, \varphi_{2}, \ldots, \varphi_{N}\right)
$$

and each fundamental parameter is expressed in terms of its average value $(\bar{\varphi})$ and the associated uncertainty $(\delta \varphi)$ :

$$
\varphi_{i}=\bar{\varphi}_{i}+\delta \varphi_{i}
$$

The resultant uncertainty of the derived parameter is obtained as follows:

$$
\delta F=\sqrt{\left(\frac{\partial F}{\partial \varphi_{1}}\right)^{2} \delta \varphi_{1}^{2}+\left(\frac{\partial F}{\partial \varphi_{2}}\right)^{2} \delta \varphi_{2}^{2}+\cdots+\left(\frac{\partial F}{\partial \varphi_{N}}\right)^{2} \delta \varphi_{N}^{2}}
$$

The sources of uncertainty were quantified either through available data and specifications, or through repeatability studies. For instance, the uncertainty associated with the pressure transducers is assessed based on transducer specifications and hysteresis analysis (slope of the linear calibration curve):

$$
P=m V+z
$$

where $V$ represents the transducer output voltage and $z$ is the intercept.

$$
\delta P=\sqrt{\left(\frac{\partial P}{\partial m}\right)^{2} \delta m^{2}+\left(\frac{\partial P}{\partial V}\right)^{2} \delta V^{2}+\left(\frac{\partial P}{\partial V}\right)^{2} \delta z^{2}}
$$

Similarly, the uncertainties associated with the following sources were identified and quantified: thermocouple temperatures, data acquisition system, sampling rates and times (Jeffries, 1995), seven-hole probe calibration, blowing-pressure fluctuations and probe position.

Figure 4.4 shows the relative uncertainty for the absolute pressure measurements for the range of pressures encountered in this experiment (60 $\mathrm{kPa}$ (a) to $150 \mathrm{kPa}(\mathrm{a}))$. As 
shown, the relative pressure uncertainty is approximately constant $(<0.30 \%)$ over this range.

As discussed in Chapter 3, seven-hole probe and Kiel probe results initially showed small differences that were later eliminated by minimizing the flow leakage into or out of the test section. However, additional sources of uncertainty were identified in the course of the investigations; these include the effects of shear flow, turbulence and Reynolds number on probe measurements, imperfect flow periodicity and the possibility of flow unsteadiness.

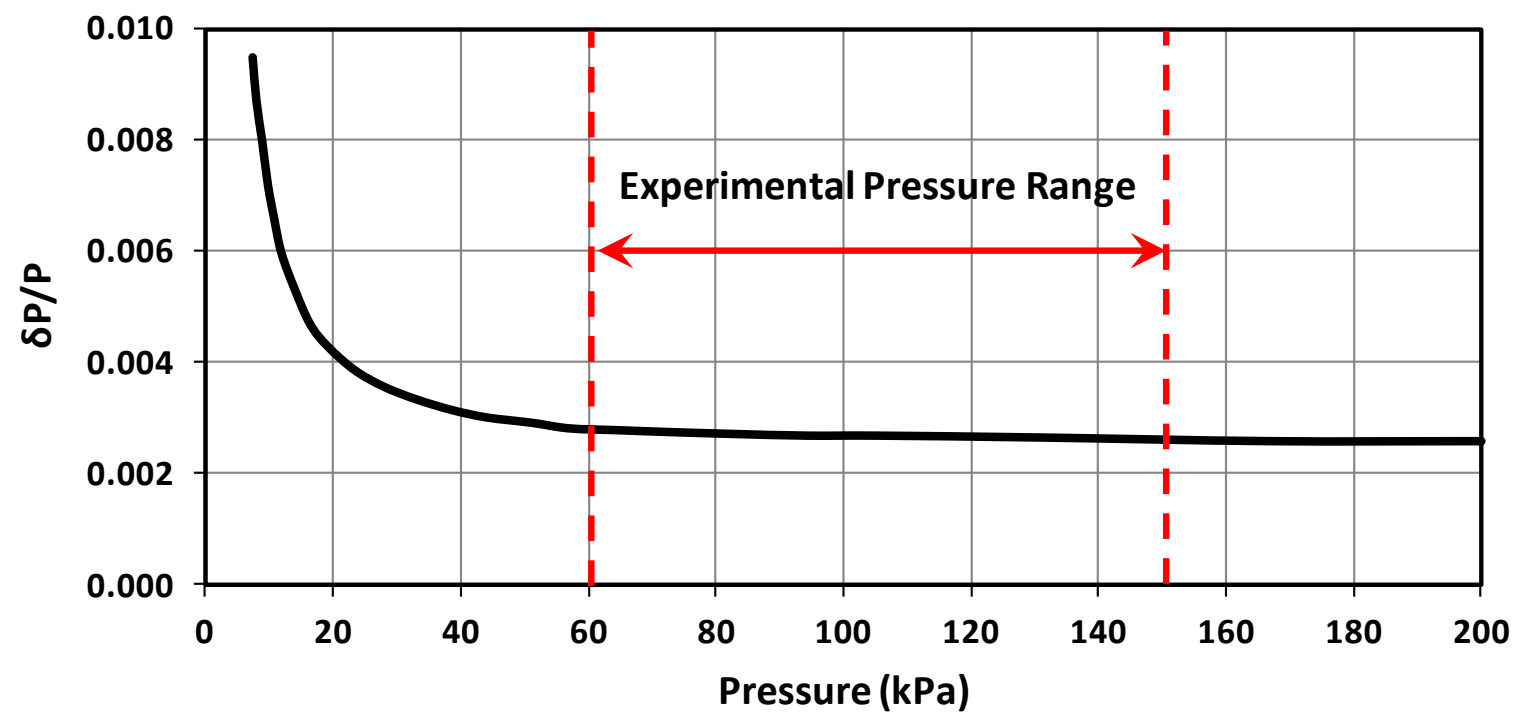

Figure 4.4 Relative pressure uncertainty for the pressure range in the high-speed wind tunnel

Scribner (2011) investigated the effects of turbulence and Reynolds number on sevenhole probe measurements in both the low-speed and high-speed wind tunnel facilities at Carleton university. The influence was found to be small in the high-speed wind tunnel since the probe is calibrated in a turbulent jet (with approximately 5\% turbulence intensity, comparable with that experienced in the test section) and the Reynolds numbers based on probe diameter are relatively large $\left(R e_{D}>15,000\right)$.

The effects of shear flow, pressure and velocity gradients across the nose of the probe, were assessed based on the seven-hole probe and Kiel probe measurements, which were found to be in very good agreement for all test cases. The influence of shear flow becomes more significant near the endwall, and therefore limits the location of the probe 
traverse close to the wall at 3\% span; the probe traverses below 3\% span showed large changes in static pressure due to wall interference effects.

The possibility of flow unsteadiness in the test section was examined by recording videos during surface flow visualization studies, conducting repeatability studies and analyzing CFD convergence histories. There was no visual evidence of low-frequency transient behaviour, associated with the movement of the vortical structures, in the surface flow visualization videos. In addition, the results from the repeatability studies were in good agreement, and the CFD convergence history did not show large oscillations, which are typically associated with flow unsteadiness. However, it should be noted that additional investigations using a fast-response probe or hot-wire anemometry would be required to quantify the level of secondary flow unsteadiness. The contribution of the higher frequency oscillations associated with trailing edge shed vortices to the overall uncertainty is accounted for by the uncertainty analysis. Finally, the effect of flow non-uniformity was examined by comparing the probe measurements for the middle three blade passages.

\subsubsection{Summary of Uncertainties}

The experimental uncertainties are summarized in Table 4.1. These represent the most conservative (i.e. largest) estimates. In particular, the flow angle and Mach number uncertainties for the seven-hole probe are larger than the values previously quoted by Corriveau (2005) for the three-hole probe.

Table 4.1 Summary of experimental uncertainties

\begin{tabular}{|c|c|c|}
\hline Parameter & Range Measured & Uncertainty \\
\hline Probe Pitchwise Position & $0-150 \mathrm{~mm}$ & $\pm 0.2 \mathrm{~mm}$ ( $\pm 0.3 \%$ span) \\
\hline Probe Spanwise Position & $0-61 \mathrm{~mm}$ & $\pm 0.4 \mathrm{~mm}$ ( $\pm 0.6 \%$ span) \\
\hline Pressure & $60-150 \mathrm{kPa}(\mathrm{a})$ & $\pm 0.3 \%$ of Measured Value \\
\hline Flow Angle & $\pm 20^{\circ}$ relative to probe & $\pm 1^{\circ}$ \\
\hline Mach Number & $0.20-0.99$ & $\pm 50,000$ \\
\hline Outlet Reynolds Number & $480,000-700,000$ & \pm 0.0005 \\
\hline Secondary Kinetic Energy Coefficient & $0.001-0.007$ & \pm 0.007 \\
\hline Loss Coefficient & $0.02-0.07$ & \pm 07 \\
\hline
\end{tabular}




\section{Chapter 5}

\section{Computational Procedures}

\subsection{Introduction}

The computational methods and procedures are presented in this chapter. The computational domains were generated using Pro/E Wildfire solid modeling software, and the surface and volume meshes were constructed using ANSYS ICEM CFD meshing software. The commercial CFD code ANSYS CFX was used for the 2D and 3D steadystate simulations. These codes are thoroughly documented in their corresponding user manuals and in the open literature. Therefore, only relevant information regarding the computational settings, the turbulence model and the transition models are provided here.

The numerical results were verified through both grid convergence and iterative convergence studies. In addition, parametric studies were performed to assess the sensitivity of the numerical results to inlet turbulence intensity and turbulence length scale. The computational results are compared and contrasted with the measurements in the subsequent chapters.

\subsection{Computational Mesh}

\subsubsection{Introduction}

ANSYS ICEM CFD (versions 11 and 12) was used to generate the computational grids. Multi-block hexahedral meshes were used for the simulations as they provide flexibility for local coarsening or refining of the mesh, as well as rapid grid convergence studies. Selecting a hexahedral mesh, however, does not reduce the computational cost since ANSYS CFX (versions 11 and 12) utilizes an unstructured solver for data structure handling. The computational investigations were performed for the following cases: 
- Inlet endwall boundary layer simulations, used for subsequent 3D simulations

- Profile flow simulations for all configurations

- Secondary flow simulations for selected cases

\subsubsection{Inlet Pre-Domain: Inlet Endwall Boundary Layers}

A computational inlet pre-domain was used to determine the spanwise variations of turbulence parameters across the inlet for the 3D simulations. The computational domain and the velocity profiles at three axial locations are presented in Figure 5.1. It should be noted that the 2D inlet pre-domain does not model the inlet convergence that is present in the wind tunnel (Figure 3.2).

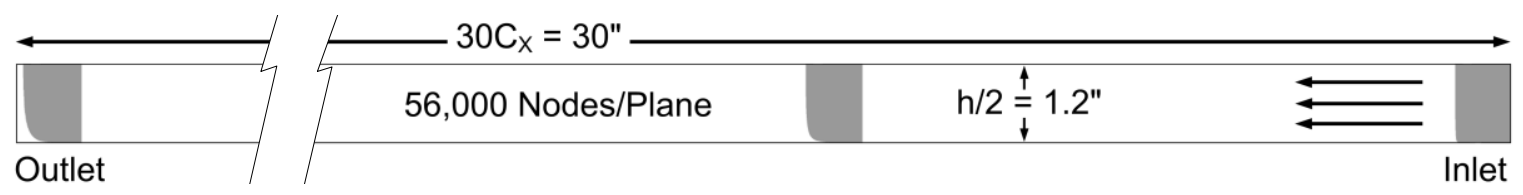

Figure 5.1 Inlet (pre-domain) configuration and computational mesh

Parametric CFD studies were conducted to determine both the length of the computational domain and the boundary conditions required to replicate the cascade-inlet boundary layers in the experiment that match the measured velocity profile, centreline turbulence intensity and turbulence length scale. However, it was not possible to match all three parameters in any single simulation and therefore only the first two parameters were matched closely. The predicted length scale variation across the span was then scaled based on the experimental value at midspan, as described in Section 5.3.2.

\subsubsection{Turbine Cascades: 2D and 3D Mesh Generation}

As a representative case, the computational domain and the multi-block $(\mathrm{H}-\mathrm{O}-\mathrm{H})$ surface mesh for SL2F are shown in Figure 5.2. The domain extends from $-1.5 C_{X}$ to 3.5 $C_{X}$ across one blade pitch. The location of the upstream boundary is selected to match the inlet traverse plane in the experiments. The outlet boundary, on the other hand, is located further downstream at $3.5 C_{X}$ to minimize its impact on the predictions and also to examine the mixing processes downstream of the cascades. The node distribution is also indicated here; the inflation layer of the O-grid contains 40 nodes, extending from the blade surface (first node height $=0.001 \mathrm{~mm}$ ) with an expansion ratio of 1.1 towards the 
outer H-block (final node height $=0.12 \mathrm{~mm}$ ). Since ANSYS CFX does not accept a twodimensional mesh, the surface mesh was extruded in the spanwise direction for both $2 \mathrm{D}$ and $3 \mathrm{D}$ simulations (to three and eighty spanwise planes, respectively). The 2D simulations model the midspan flow, whereas the 3D simulations cover the area between the endwall and 50\% span.
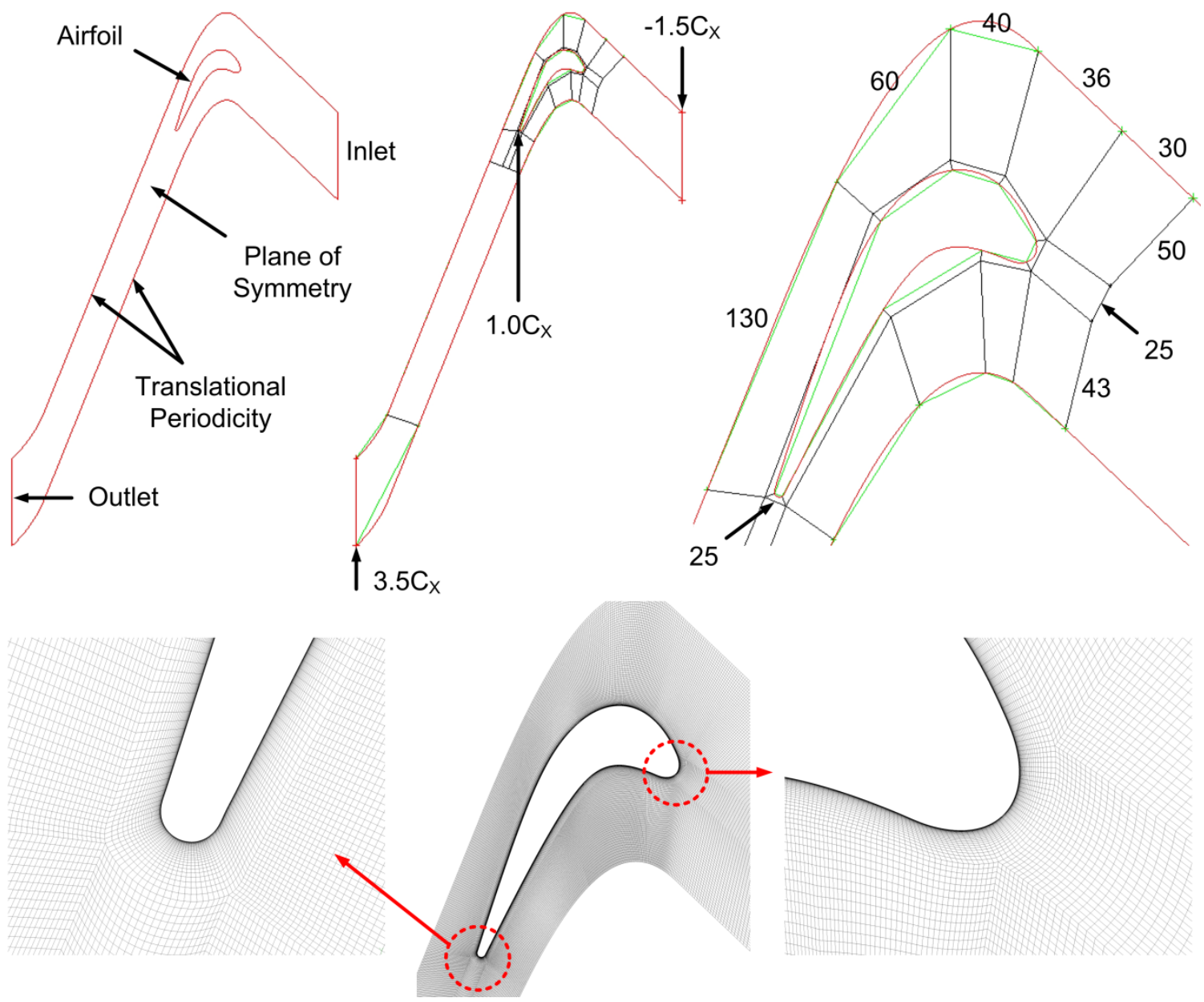

Figure 5.2 Computational domain and 2D surface mesh for SL2F

Preliminary studies exhibited a strong correlation between mesh quality and numerical convergence. Several criteria were used to assess the mesh quality:

- $\quad$ Determinant $>0.8$

- $\quad$ Airfoil surface and endwall $\mathrm{y}^{+}<2$

- $\quad$ Free-stream edge expansion ratio $<1.3$

- $\quad$ Minimum face angle $>30^{\circ}$

- $\quad$ Near-wall edge expansion ratio $\approx 1.1$ 
- Volume change between adjacent volumes $<2$

The mesh quality criteria, which are more conservative than the CFX requirements, were set based on the best practice guidelines for turbomachinery CFD simulations from the available literature. The quality of the final mesh is in part constrained by the shape of the computational domain. The selected geometry may dictate the minimum face angle; for example, the exit flow angle for SL2F is approximately $70^{\circ}$, which could result in a minimum face angle of $20^{\circ}$ if the domain outlet is aligned with the exit flow angle. Therefore, the shape of the computational domain was optimized for each case in order to obtain the highest quality mesh (e.g. this required a curved outlet for SL2F). It was also determined that for the large levels of flow turning encountered here (e.g. $112^{\circ}$ for SL2F), the one-to-one node connectivity on the periodic surfaces would result in highly skewed elements. As a result, the simulations were set up to use the General Grid Interface (GGI) instead. The final mesh was further refined to ensure smooth transitions between the blocks; this is especially apparent near the leading edge and the trailing edge regions in Figure 5.2 and Figure 5.3, which shows the 3D surface mesh for SL2C. The 2D and 3D mesh statistics are summarized in Table 5.1.
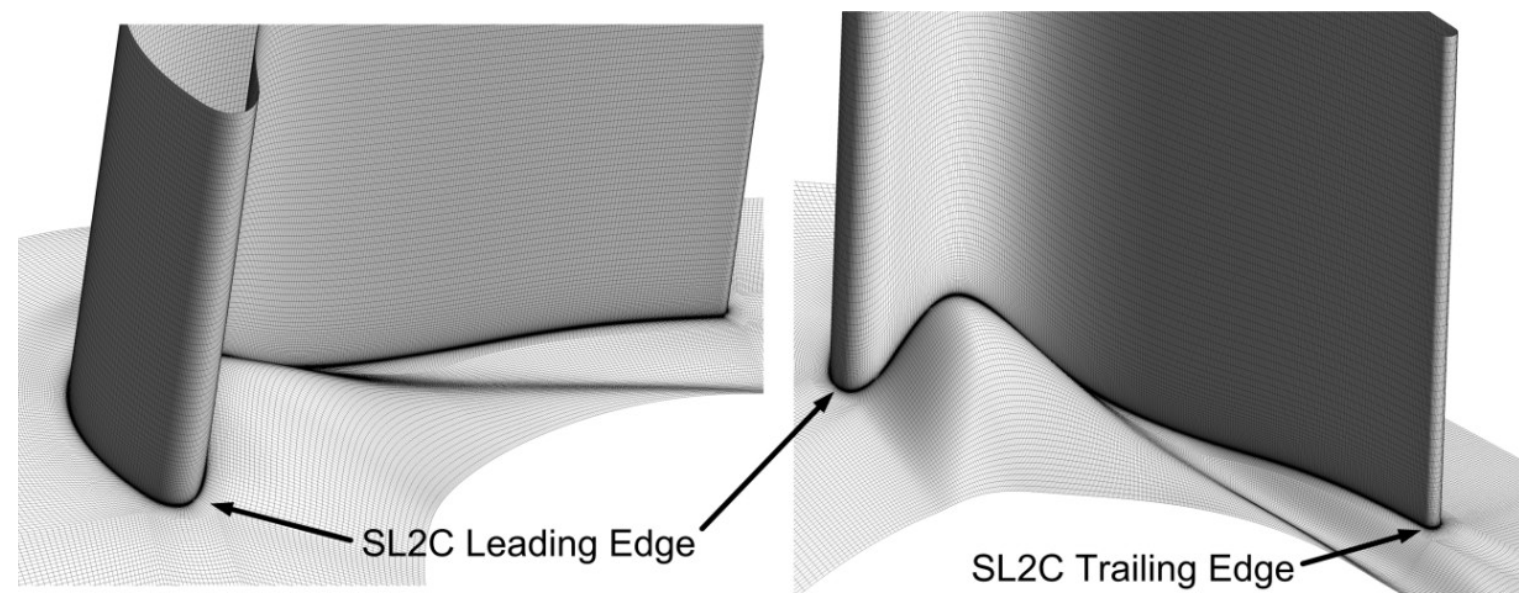

Figure 5.3 3D surface mesh for SL2C

\subsection{Reynolds-Averaged Navier-Stokes Solver: ANSYS CFX}

\subsubsection{General Procedures}

Solving the transonic turbine flow field requires the simultaneous solutions of five conservation equations: continuity, $\mathrm{x}-, \mathrm{y}-$ and $\mathrm{z}$-momentum, and energy, plus one 
Table 5.1 Final mesh statistics

\begin{tabular}{|l|c|c|c|c|c|}
\hline \multicolumn{7}{|c|}{ 2D Simualtions } \\
\hline & SL1F & SL2F & SL3F & SL4F & SL2P \\
\hline Number of Nodes / Plane & 69,000 & 77,000 & 61,000 & 76,000 & 76,000 \\
\hline \multicolumn{7}{|c|}{ 3D Simualtions } \\
\hline Total Number of Nodes & SL1F & SL1C & SL2F & SL2C & SL2P \\
\hline Total Number of Elements & $4.72 \times 10^{6}$ & $5.04 \times 10^{6}$ & $5.40 \times 10^{6}$ & $4.97 \times 10^{6}$ & $5.25 \times 10^{6}$ \\
\hline Total Number of Faces & $4.59 \times 10^{6}$ & $4.91 \times 10^{6}$ & $5.26 \times 10^{6}$ & $4.84 \times 10^{6}$ & $5.12 \times 10^{6}$ \\
\hline
\end{tabular}

equation of state. ANSYS CFX utilizes an implicit, finite-volume solver, which is second-order accurate in space (through specifying a blend factor of one in the solver). The default algebraic multi-grid scheme was also used for all the simulations to improve the numerical convergence. Typically, a relatively fine mesh is required to obtain approximately grid-independent results. The multi-grid scheme constructs coarser meshes initially based on the gradients in the hydrodynamic equations, and subsequently refines the mesh in several steps. The solver cycles back and forth between the coarse and finer grids during the convergence.

Turbulence closure was achieved using the two-equation Shear Stress Transport (SST) turbulence model (Menter, 1994). The pseudo-second-order "high resolution" scheme in ANSYS CFX was used to discretize the turbulence transport equations since a true second order scheme was not available. Although several different turbulence models are available in CFX, the SST model was selected since it is robust and computationally inexpensive, and it has been validated for turbomachinery flows with boundary layer separation. The SST turbulence model is a combination of the $k-\omega$ model in the near-wall regions (boundary layers) and the $k-\varepsilon$ model in the outer regions. The two-equation turbulence models, based on the Boussinesq approximation, account for history effects, such as convection and diffusion of turbulence energy; however, they do not account for turbulence anisotropy and instead compute an isotropic eddy viscosity distribution within the flow field. As shown by Menter (1994), the two-equation turbulence models typically overpredict the turbulence kinetic energy (TKE) production in regions of large flow acceleration (e.g. approaching the suction peak) or large flow deceleration (e.g. the leading edge region and the aft end of suction surface). The SST 
turbulence model uses $T K E$ production limiters to manage this limitation (ANSYS CFX Manual, 2010).

Higher order turbulence models, such as the Reynolds stress model, may theoretically provide superior predictions by calculating the anisotropy in the flow field. However, it was not possible to obtain a converged solution using the Reynolds stress turbulence model (a known issue documented in the ANSYS CFX Manual, 2010).

The $\gamma-R e_{\theta}$ transition model (Langtry and Menter, 2005) was used to predict boundary layer transition for the $2 \mathrm{D}$ simulations. The $\gamma-R e_{\theta}$ transition model is optimized for use with the SST turbulence model. The model is based on the locally-formulated transport equation for intermittency to trigger transition. The transition onset criteria are determined in terms of the free-stream turbulence intensity and the momentum thickness Reynolds number (ANSYS CFX Manual, 2010).

\subsubsection{Simulation Settings and Boundary Conditions}

The boundary conditions in the computations were defined such to replicate the experimental measurements wherever possible. Air (ideal gas) with constant specific heats was selected as the working fluid for the simulations. The variations in dynamic viscosity with temperature were modeled using Sutherland's formula. The reference pressure and the reference temperature were set to $101325 \mathrm{~Pa}$ and $25^{\circ} \mathrm{C}$, respectively. These values are comparable with the encountered pressure and temperature ranges in the experiments:

- Inlet centerline total pressure: $118 \mathrm{kPa}(\mathrm{a})\left(\right.$ low $\left.M_{2}\right)$ to $145 \mathrm{kPa}$ (a) (high $M_{2}$ )

- Outlet static pressure: $62 \mathrm{kPa}(\mathrm{a})\left(\right.$ high $M_{2}$ ) to $93 \mathrm{kPa}$ (a) (low $M_{2}$ )

- Ambient (laboratory) air temperature: $10^{\circ} \mathrm{C}$ (winter) to $30^{\circ} \mathrm{C}$ (summer)

- Static air temperature: $-40^{\circ} \mathrm{C}$ (cascade outlet) to $15^{\circ} \mathrm{C}$ (inlet)

The locations of the boundary conditions are shown in Figure 5.2. The boundary conditions were specified as follows:

- Inlet: uniform total temperature, total pressure and flow angles for the 2D simulations; inlet endwall boundary layer profile for the 3D simulations; inlet turbulence parameters as discussed below

- Airfoil: no slip wall, smooth and adiabatic 
- Midspan: plane of symmetry

- Endwall: no slip wall, smooth and adiabatic for the 3D simulations

- Translational periodic boundaries: conservative interface fluxes for all parameters

- Outlet: area-averaged static pressure to match the experimental outlet isentropic Mach number

Five different parameters were required to fully specify the inlet boundary conditions: total pressure, total temperature, flow angle, turbulence intensity and length scale. The first three parameters were obtained directly from the experiments, assuming constant total temperature and constant flow angle (in both pitchwise and spanwise directions) across the inlet plane. The free-stream turbulence intensity and the integral length scale at the inlet centreline have also been documented by Corriveau (2005), and used here for the $2 \mathrm{D}$ simulations $\left(T u_{M S}=4 \%\right.$ and $\left.L_{t, M S}=15 \mathrm{~mm}\right)$. The $2 \mathrm{D}$ inlet pre-domain was used to determine the spanwise variations of the turbulence quantities across the inlet endwall boundary layer. The computed and measured Mach number distributions for inlet endwall boundary layers at the design Mach number are shown in Figure 5.4, along with the computed distributions of turbulence intensity and length scale.
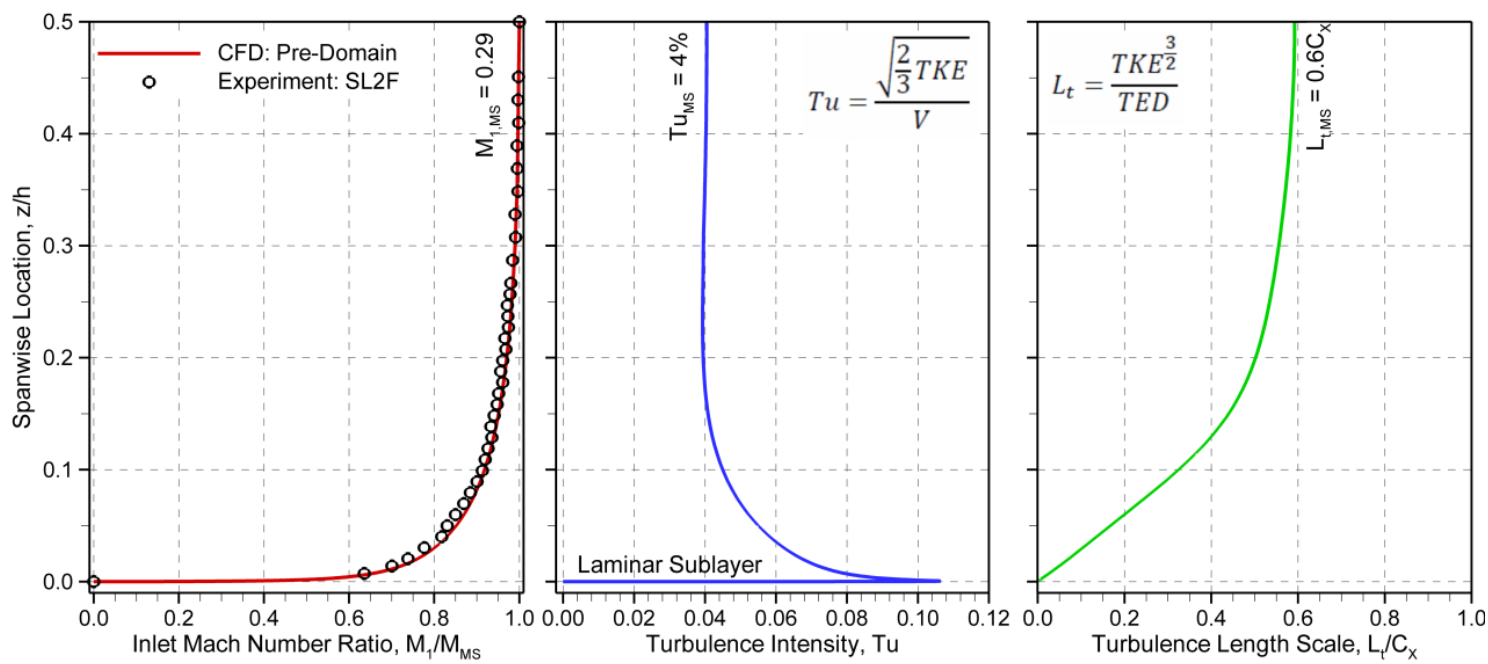

Figure 5.4 CFD results at the outlet of the pre-domain: Mach number ratio, turbulence intensity and length scale distributions $\left(M_{2, i s}=0.8\right)$

As indicated in Figure 5.4, the turbulence intensity is a function of both turbulence kinetic energy $(T K E)$ and local mean flow velocity $(V)$. The local turbulence intensity 
remains constant at approximately 0.04 from $50 \%$ to $15 \%$ span. The mean velocity decreases in the boundary layer towards the endwall, whereas the turbulence kinetic energy increases. Below $15 \%$ span, $T u_{\text {local }}$ increases from 0.04 to about 0.1 due to the significant drop in local mean velocity. Within the laminar sublayer, the turbulence kinetic energy is dissipated by viscous forces, resulting in a very rapid decline in turbulence intensity. The turbulence length scale, which is a function of turbulence kinetic energy and turbulence eddy dissipation (TED), decreases slightly from midspan to about $15 \%$ span. Below $15 \%$ span, the turbulence eddy dissipation increases at a faster rate than the turbulence kinetic energy. As a result, the length scale decreases almost linearly from $15 \%$ span towards the endwall. The boundary layer profiles, shown in Figure 5.4, were used for all subsequent 3D simulations at the design Mach number.

\subsection{Convergence Criteria and Convergence Analysis}

\subsubsection{D Convergence Analysis}

Grid convergence and iterative convergence were examined to verify the CFD predictions. Several steps were taken in order to obtain optimal convergence. The simulations were initiated as fully turbulent with first-order spatial accuracy. The results of the preliminary simulations were then used as the initial guess at each subsequent step: setting second-order spatial accuracy, implementing the transition model, and gradually decreasing the maximum timescale and the timescale factor. The goal was to filter out the low-frequency oscillations (e.g. suction-surface flow separation) without capturing the high-frequency unsteadiness (e.g. trailing edge vortex shedding). During this process, both mean (RMS) and maximum (MAX) residuals were monitored. The convergence history for one of the 2D simulations for SL2F at design point is presented in Figure 5.5; other 2D simulations exhibited similar characteristics.

The red arrows indicate two steps of timescale reduction for this particular simulation: the maximum timescale was initially set to 0.1 milliseconds and subsequently reduced to $0.01 \mathrm{~ms}$ after 380 iterations, and to $0.001 \mathrm{~ms}$ after 2280 iterations; the MAX residuals drop sharply as the maximum timescale is reduced. The initial timescale was selected based on the minimum time for flow passing through the computational domain (about $0.4 \mathrm{~ms}$ at Mach 1.0). The mixed-out loss coefficients and flow angles were 
obtained from the results files at four separate instances to assess the iterative convergence. As shown in Figure 5.5, these values do not change after about 400 iterations.

Ideally, the maximum solution residuals should be less than $10^{-6}$ for well-behaved steady-state simulations. However, the flow field within the linear turbine cascade is subjected to several sources of unsteadiness. The main sources of unsteadiness for the 2D simulations are associated with surface flow separation and transition, shock wave/boundary layer interaction, and trailing edge vortex shedding. The 3D simulations may exhibit additional transient tendencies associated with the formation of the vortical structures, and their interactions with both endwall and suction surface boundary layers. The main goal of the steady-state RANS simulations is to provide a time-averaged picture of the flow field, which thus requires dampening of the unsteady characteristics. This is typically accomplished by coarsening the mesh, which introduces artificial numerical dissipation in regions of flow unsteadiness, such as near the trailing edge. In other words, the final numerical solution requires a trade-off between accuracy and robustness. In Figure 5.5, the maximum residuals for $\operatorname{Re}_{\theta}$ show rather large fluctuations; however, the fluctuations are limited to a few nodes within the domain, and do not significantly affect the predictions of integrated quantities, such as loss coefficient and flow angles.

The 2D grid convergence study was performed for SL2F since it is both highly loaded and has high flow turning. Five different grids with varying densities were generated for this study as indicated in Figure 5.6. Additional coarser grids were generated with node counts below 40000; however, the results are not shown here since the node reduction adversely affected the mesh quality, and thus the numerical convergence. The boundary layer mesh (inflation layer) was not adjusted significantly due to the strict CFX grid quality requirements for use with the SST turbulence model. Therefore, the grid sensitivity study was carried out by primarily adjusting the number of free-stream nodes. Raising the node count does not affect the total pressure deficit across the pitch noticeably in Figure 5.6. The integrated loss coefficients and the exit flow angles also remain constant for the three largest grids. All subsequent simulations were performed on the second largest grid with approximately 77000 nodes per 2D plane. 

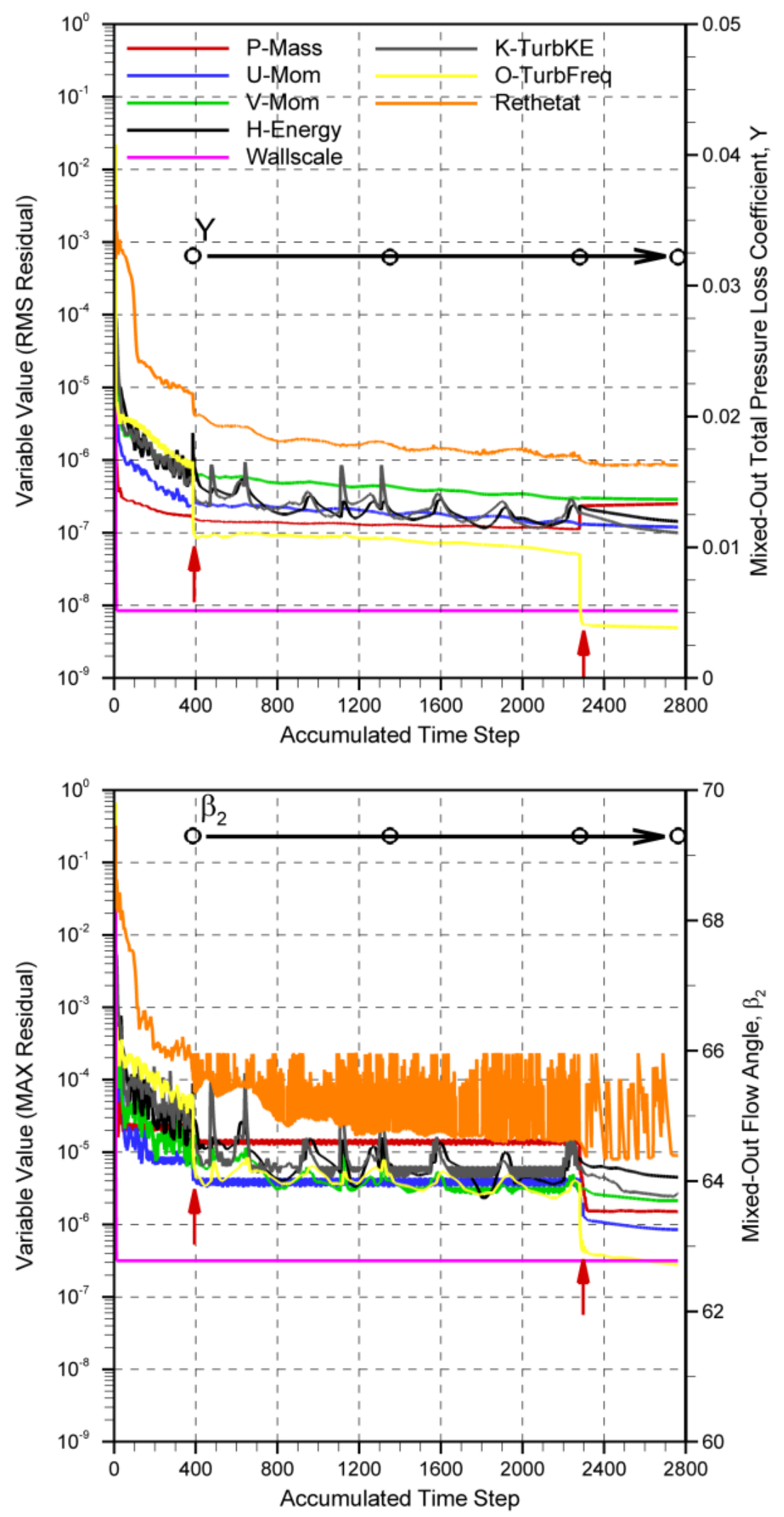

Figure 5.5 Convergence history for one of the 2D simulations for SL2F: RMS residuals (top) and MAX residuals (bottom) 
An additional iterative convergence study was conducted to assess the final 2D predictions for the selected grid. The total pressure loss coefficients and flow angles at several axial locations are shown in Figure 5.7, which depict the diffusion and mixing processes downstream of the cascade.
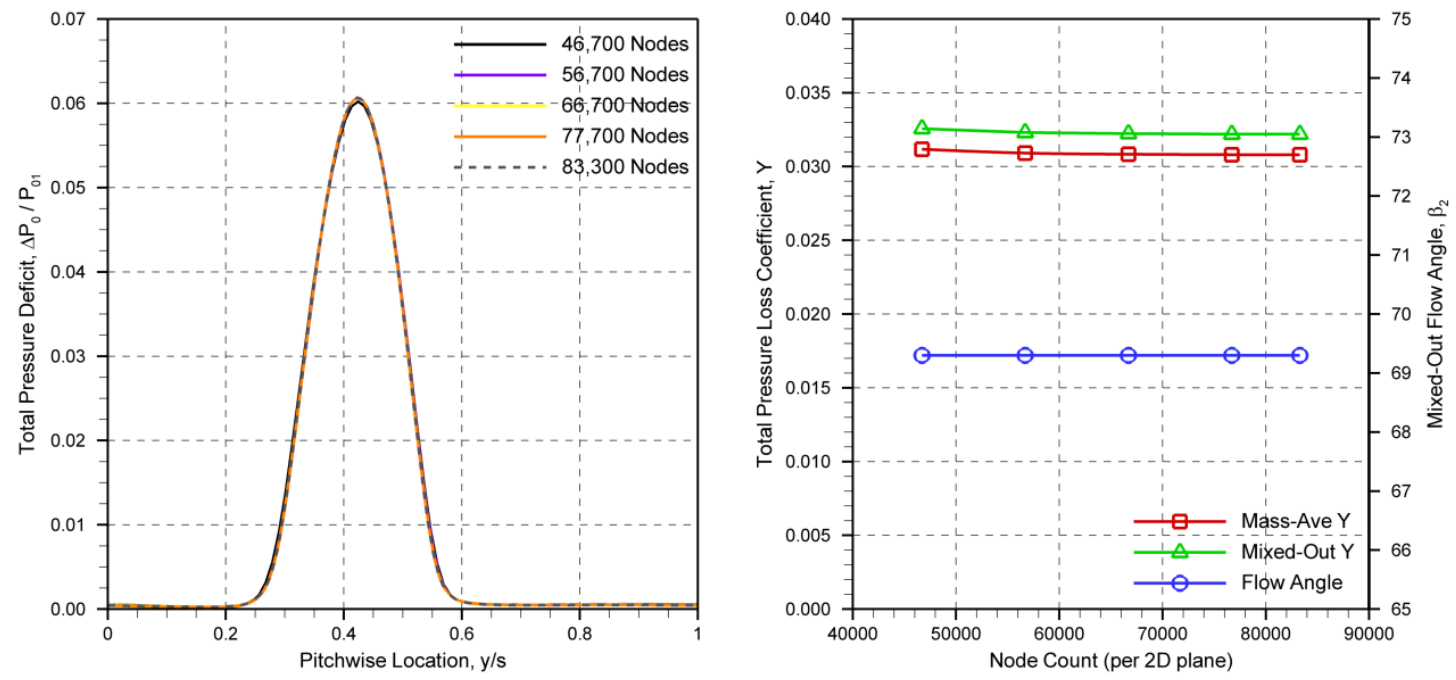

Figure 5.6 Grid convergence study for SL2F (2D simulations): total pressure deficit at 2.0 $C_{X}$ (left) and integrated quantities (right)
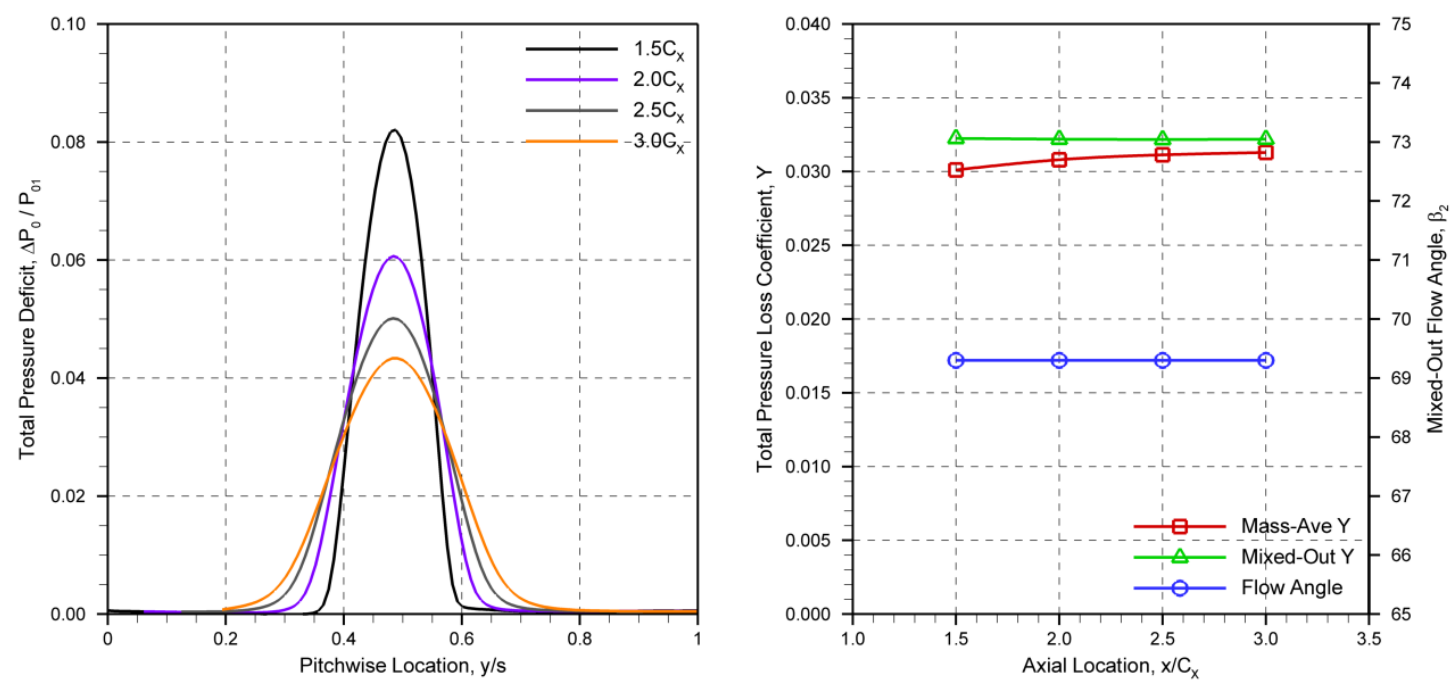

Figure 5.7 Iterative convergence study for SL2F (2D simulation): total pressure deficit (left), loss coefficients and exit flow angles (right)

The mass-averaged loss coefficient increases downstream of the airfoil, whereas the mixed-out loss coefficient remains constant and independent of the extraction plane 
location; this is to be expected since ANSYS CFX utilizes a finite-volume solver, equivalent to the control-volume analysis used by the mixing-out calculation. On the other hand, if the simulation is not fully converged, the mixed-out results are influenced by the location of the extraction plane. This additional verification was performed on all 2D cases examined in the present study.

\subsubsection{D Convergence Analysis}

As discussed in Section 5.2, the 2D surface mesh was extruded from midspan to the endwall for the 3D simulations. Approximately 100 spanwise planes, including inflation layers near the endwall, were required to obtain a high-quality mesh, corresponding to about eight million nodes for the entire domain. The 3D transitional simulations, however, exhibited poor numerical convergence in the form of oscillating residuals (MAX residuals $\approx 10^{-3}$ ). Additional mesh modifications and under-relaxation did not improve the results, and therefore the transition model was excluded from the 3D simulations. The mesh was subsequently coarsened in accordance with the less stringent CFX requirements for fully-turbulent simulations; the final node count was approximately 5 million. The final 3D mesh is considerably more refined than the grids typically utilized for the analysis of turbine blade rows in the industry (full-span simulations with mesh refinements near both endwalls):

- Praisner et al. (2007): 800,000 nodes

- Pullan and Harvey (2008): 790,000 nodes

- Schuepbach et al. (2009): 1,500,000 nodes

- Germain et al. (2010): 1,500,000 nodes

- Panchal et al. (2011): 1,800,000 nodes

The numerical convergence was improved by turning off the transition model for the $3 \mathrm{D}$ simulations (RMS residuals $\approx 10^{-5}$ and MAX residuals $\approx 10^{-4}$ ). However, the $3 \mathrm{D}$ numerical predictions should be examined with some caution due to lack of transition prediction. The results of the 3D grid convergence study are presented in Figure 5.8 and Table 5.2.

As shown, reducing the grid count from 5.4 million to 4.1 million has a very small impact on the predictions: the integrated loss coefficient changes by less than $0.2 \%$. In 
addition, the mass flows and mass-averaged total temperatures (only available from CFD) remain constant between the inlet and outlet planes. The larger grid size was selected for the simulations since it has better grid quality.

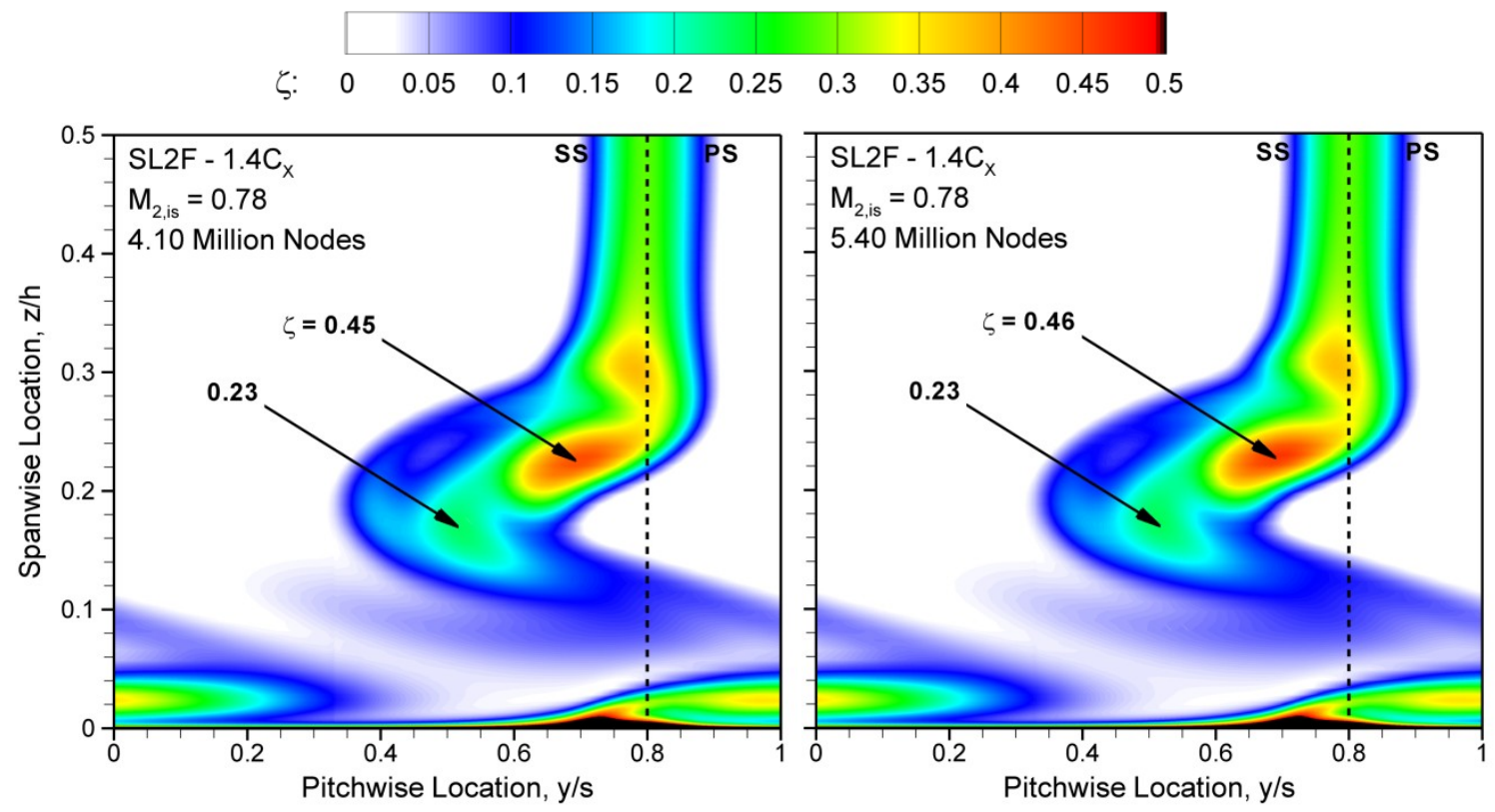

Figure 5.8 3D grid convergence study (SL2F): colour floods of energy loss coefficient

Table 5.2 3D grid convergence study: mass-averaged results (SL2F)

\begin{tabular}{|c|c|c|c|c|c|}
\hline $\begin{array}{c}\text { Number of } \\
\text { Nodes }\end{array}$ & $\begin{array}{c}\text { Energy Loss } \\
\text { Coefficient }\end{array}$ & $\begin{array}{c}\text { Pitchwise Flow } \\
\text { Angle (deg) }\end{array}$ & $\begin{array}{c}\text { Spanwise Flow } \\
\text { Angle (deg) }\end{array}$ & $\begin{array}{c}\text { Outlet Total } \\
\text { Temperature (K) }\end{array}$ & $\begin{array}{c}\text { Secondary Kinetic } \\
\text { Energy Coefficient }\end{array}$ \\
\hline 4.10 million & 0.0660 & 67.52 & 1.55 & 285.00 & 0.0123 \\
\hline $\mathbf{5 . 4 0}$ million & 0.0661 & 67.51 & 1.58 & 285.00 & 0.0126 \\
\hline
\end{tabular}

\subsection{Sensitivity of 2D CFD Predictions to Inlet Turbulence and}

\section{Transition Modeling}

\subsubsection{Introduction}

The influence of the inlet turbulence parameters on the 2D simulations are examined in this section. The conclusions drawn from the $2 \mathrm{D}$ simulations were then taken to apply to the $3 \mathrm{D}$ computations as well.

ANSYS CFX requires the specification of two independent turbulence parameters at the inlet plane. For the present study, the inlet turbulence intensity and the turbulence length scale were specified based on the experimental measurements by Corriveau 
(2005). Other inlet turbulence quantities, calculated by CFX, include: turbulence kinetic energy $(T K E)$, turbulence eddy frequency $(T E F)$, turbulence eddy dissipation $(T E D)$ and eddy viscosity ratio $(E V R)$. The transitional predictions are also compared and contrasted with the fully-turbulent predictions to quantify the effects of the transition model on profile losses.

As shown in Table 5.3 the inlet eddy viscosity ratio was varied from 10 to 1000 , corresponding to inlet turbulence length scales between $0.3 \mathrm{~mm}$ and $27 \mathrm{~mm}$. The inlet turbulence intensity was varied between $2 \%$ and $4 \%$ for both fully turbulent and transitional simulations, as shown in Table 5.4.

Table 5.3 Inlet turbulence length scale variations (transitional simulations)

\begin{tabular}{|c|c|c|c|c|c|c|c|}
\hline Inlet Turbulence Intensity, $\boldsymbol{T u}$ & \multicolumn{7}{|c|}{0.04} \\
\hline Inlet Turbulence Length Scale, $\boldsymbol{L}_{\boldsymbol{t}}(\mathbf{m m})$ & 0.3 & 1.4 & 2.7 & 6.8 & 14.9 & 20.3 & 27.0 \\
\hline Inlet Eddy Viscosity Ratio, $\boldsymbol{E} \boldsymbol{V} \boldsymbol{R}$ & 10 & 50 & 100 & 250 & 550 & 750 & 1000 \\
\hline
\end{tabular}

Table 5.4 Inlet turbulence intensity variations $\left(L_{t}=15 \mathrm{~mm}\right)$

\begin{tabular}{|c|c|c|c|c|c|c|}
\hline Turbulence Model & \multicolumn{4}{|c|}{ Shear Stress Transport } \\
\hline Transition Model & \multicolumn{3}{|c|}{ n/a } & \multicolumn{3}{|c|}{$\gamma-\operatorname{Re}_{\vartheta}$} \\
\hline Inlet Turbulence Intensity, $\boldsymbol{T} \boldsymbol{u}$ & 0.02 & 0.04 & 0.06 & 0.02 & 0.04 & 0.06 \\
\hline
\end{tabular}

\subsubsection{Sensitivity of 2D Predictions to Inlet Eddy Viscosity Ratio}

The specified inlet turbulence length scale and the computed inlet eddy viscosity ratio are directly related; the following ranges of parameters were investigated here:

- the medium turbulence setting in CFX: $E V R=10 \Rightarrow L_{t}=0.3 \mathrm{~mm}$

- $\quad$ the high turbulence setting in CFX: $E V R=100 \Rightarrow L_{t}=2.7 \mathrm{~mm}$

- the integral length scale in the experiments: $L_{t}=15 \mathrm{~mm} \Rightarrow E V R=550$

- typical value in HP turbines (CFX manual, 2010) $: E V R=1000 \Rightarrow L_{t}=27 \mathrm{~mm}$

The effects of inlet eddy viscosity ratio on the turbulence decay are presented in Figure 5.9. The results extend from the inlet of the computational domain $\left(-1.5 C_{X}\right)$ towards the leading edge plane $\left(-0.25 C_{X}\right)$. In the experiments, the turbulence generating grid is installed about 60 axial chord lengths upstream of the cascade and normal to the flow direction. Therefore, the TKE decay in the free stream should be small across the relatively short distance $\left(1.25 C_{X}\right)$ upstream of the airfoil, compared with distance from 
the grid. However, the default settings in CFX $(10<E V R<100)$ produce very large decays for all turbulence parameters in Figure 5.9.

The effects of $E V R$ on the airfoil surface skin friction coefficients $\left(C_{f}\right)$ and the total pressure loss coefficients $(Y)$ are presented in Figure 5.10. The inlet eddy viscosity ratio clearly affects both suction surface and pressure surface boundary layers. The differences in these plots, particularly apparent on the suction surface, may be associated with the transition and separation behaviour induced by the free-stream TKE.
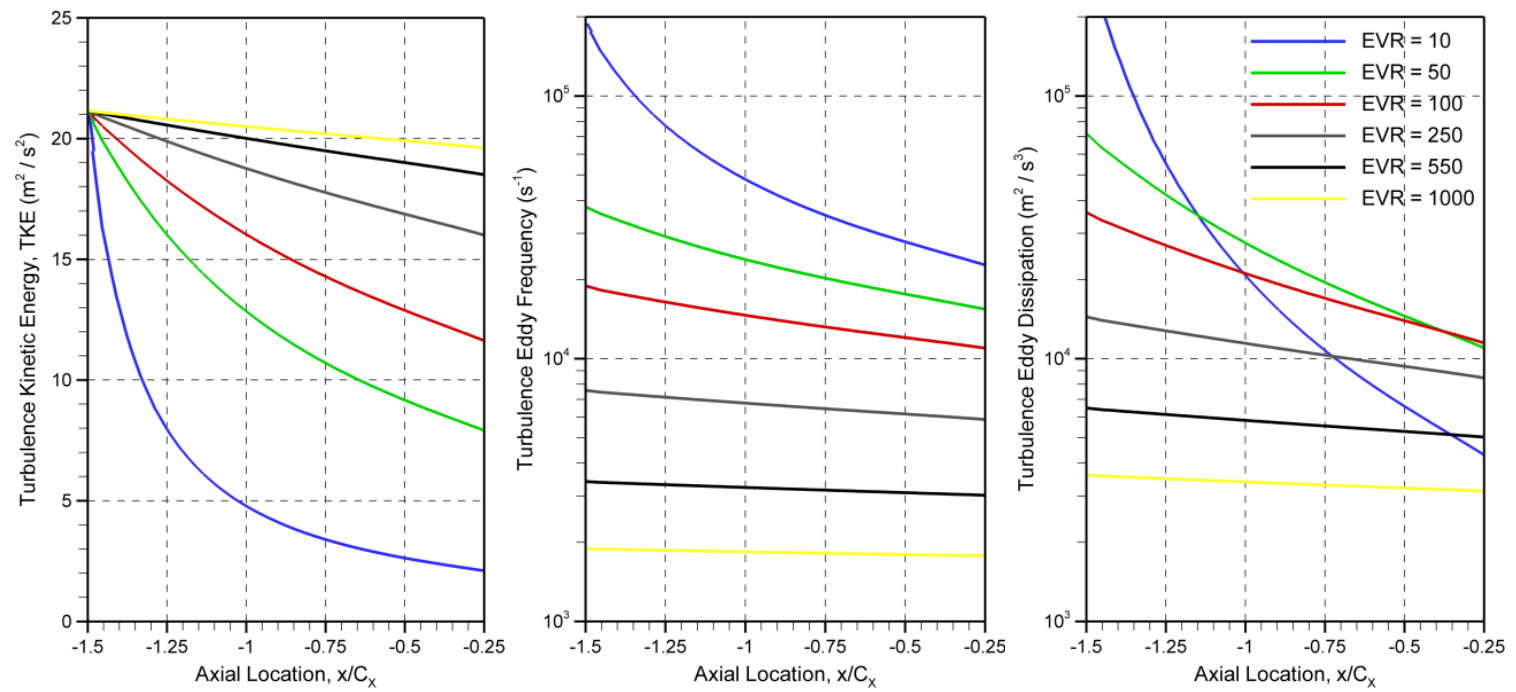

Figure 5.9 Effects of inlet eddy viscosity ratio $(E V R)$ on turbulence decay
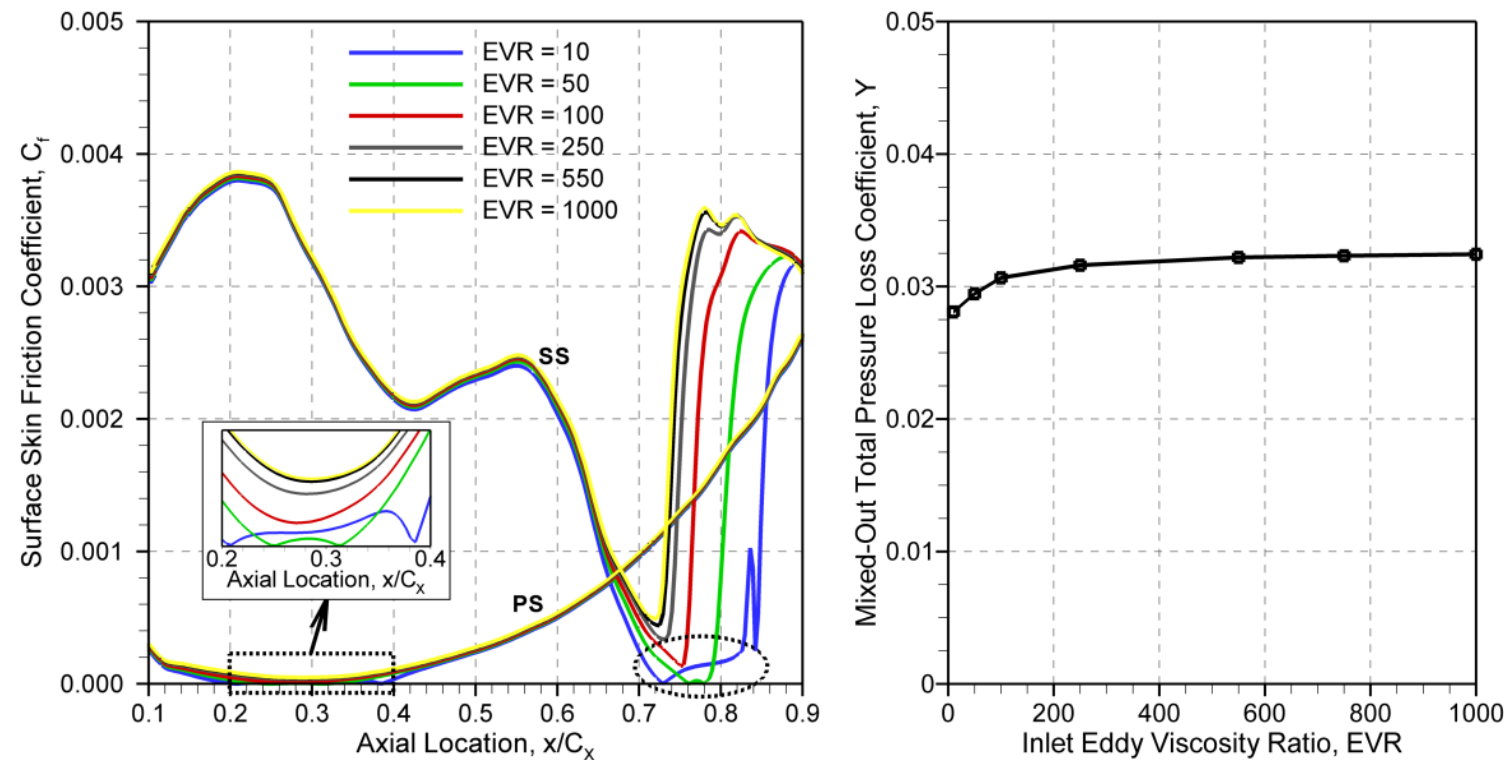

Figure 5.10 Effects of inlet eddy viscosity ratio on skin friction coefficients $\left(C_{f}\right)$ and mixedout profile loss coefficients for SL2F $\left(M_{2, i s}=0.8\right)$ 
The results from the three largest eddy viscosity ratios (250, 550 and 1000) are in good agreement as the boundary layers likely undergo bypass transition following the suction peak (boundary layers are predicted to remain attached). As the inlet eddy viscosity ratio is reduced below 250, the surface skin friction coefficient decreases and eventually drops to zero, indicative of flow separation. The separation was confirmed from the velocity vector plots. Similar trends emerge on the pressure surface: the skin friction coefficients decrease as EVR is reduced from 550 to 100, and this is followed by flow separation at lower values of $E V R$.

The loss coefficients in Figure 5.10 become nearly constant for inlet eddy viscosity ratios greater than about 250. The predicted losses, however, drop as EVR is reduced below 250, mainly due to the lower skin friction coefficients on the aft part of the suction surface; decreasing the EVR from 550 to 10 reduces the predicted loss coefficient by about $13 \%$. The inlet turbulence length scale was set to $15 \mathrm{~mm}$ for all subsequent simulations, which results in an inlet eddy viscosity ratio of 550 .

\subsubsection{Sensitivity of 2D Predictions to Inlet Turbulence Intensity}

Although the inlet turbulence intensity $(T u)$ was not varied in the experiments, the effects of changing $T u$ were briefly examined in the computations. In an engine environment, the free-stream turbulence intensity varies significantly depending on the location: $\mathrm{Tu}$ ranges from about $20 \%$ in the combustors to about $5 \%$ in the LP turbine stages. The effects of $T u$ variation on the predicted surface skin friction coefficients are presented in Figure 5.11.

For the transitional simulations, reducing the inlet turbulence intensity results in lower suction surface skin friction coefficients, primarily downstream of the suction peak. At 2\% turbulence intensity, there is evidence of separation bubbles on both pressure and suction surfaces, whereas all other cases in Figure 5.11 display fully attached boundary layers. In contrast to the transitional predictions, the $C_{f}$ values increase only slightly with $T u$ for the fully turbulent simulations (for the range examined here).

The effects of $T u$ variation on the loss coefficients are also presented in Figure 5.11. The turbulent simulations predict higher losses than the transitional simulations as 
expected from the larger surface skin friction coefficients. The loss coefficients increase almost linearly with inlet turbulence intensity; for the turbulent predictions, this loss increase is mainly due to the higher dissipation rates in the suction surface boundary layers. For the transitional simulations, decreasing the turbulence intensity from 0.04 to 0.02 reduces the predicted losses by about $11 \%$ : the additional entropy generation by the separation bubbles are offset by overall lower dissipation rates in the boundary layers. Of course, it is also conceivable that further $T u$ reductions (e.g. $T u=0.5 \%$ for clean wind tunnels) may result in longer separation bubbles or blade stall, and higher losses. The inlet turbulence intensity was set to $4 \%$ for all subsequent simulations since this is the level present in the experimental measurements.
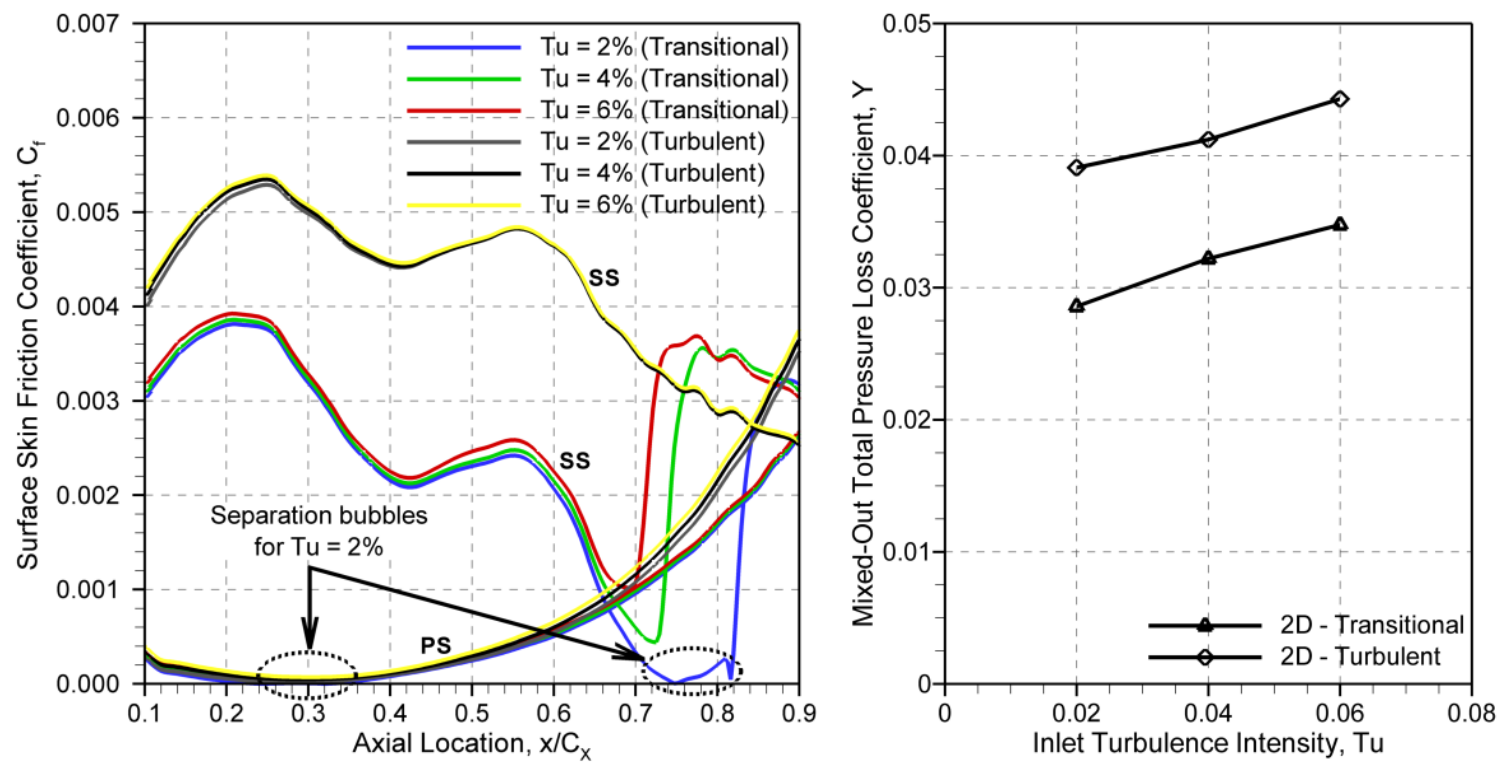

Figure 5.11 Effects of inlet turbulence intensity $(T u)$ on skin friction coefficients $\left(C_{f}\right)$ and mixed-out profile loss coefficients for $\operatorname{SL2F}\left(M_{2, i s}=0.8\right)$ 


\section{Chapter 6}

\section{Midspan Airfoil Loading Distributions and Profile Losses}

\subsection{Introduction}

As discussed in Chapter 3, the aerodynamic performance of five different turbine airfoils with different levels of flow turning $(\theta)$ and Zweifel coefficient $(Z w)$ were examined in the current project. The airfoil geometries represent modern, moderatelyloaded to highly-loaded designs with the goals of minimizing both profile losses by avoiding flow separation at midspan and secondary losses by utilizing mid- or aft-loaded configurations. The midspan flow aerodynamics of these airfoils were examined both experimentally and numerically, and the results are presented in this chapter prior to discussing the secondary flow characteristics. This approach seems appropriate since secondary losses are typically defined as the difference between row losses and profile losses, which are assumed, although incorrectly, constant across the span. Furthermore, the secondary losses are also influenced by the state of the blade surface boundary layers (laminar, transitional, turbulent or separated flow) due to the interactions of the boundary layers with the vortical structures in the secondary flow. In the present study, the outlet Mach numbers were kept subsonic to avoid the formation of strong shock waves within the blade passages. Therefore, the effects of shock waves on profile losses, previously investigated by Jouini (2000) and Corriveau (2005), are not re-examined here.

The airfoil loading distributions are presented and compared to assess their influence on loss generation within the blade surface boundary layers. The airfoil loading distribution also affects the formation of the secondary flows, and thus the secondary losses, as will be discussed in the next chapters. The experimental measurements are compared to the CFD predictions at various Mach numbers; these comparisons were also 
used to set the wind tunnel operating points. In this context, the influence of off-design Mach numbers on loading distributions and profile losses is examined both experimentally and numerically. Raising the exit Mach numbers in the experiments results in higher Reynolds numbers since the ejector-diffuser assembly is not employed for the present study. Therefore, an additional numerical study is conducted to assess the influence of Reynolds number variations on the midspan flow characteristics.

\subsection{Summary of Cascade Parameters at Design and Off-Design Conditions}

The turbine airfoils in this study were split into two categories: low flow turning (SL3 and SL4) and high flow turning (SL1, SL2 and SL2P). The geometric parameters of the cascades were presented in Chapter 3. Table 6.1 summarizes the aerodynamic parameters of the turbine cascades at design conditions $\left(M_{2, i s}=0.8\right)$, as obtained from the experiments (mixed-out flow parameters at midspan). The changes in the aerodynamic characteristics due to off-design Mach number operation (applied only to SL1, SL2 and SL2P) are summarized in Table 6.2.

The inlet turbulence quantities quoted here are based on the measurements by Corriveau (2005); these were found to be approximately constant for the range of Mach numbers and Reynolds numbers examined (see Chapter 3). The inlet endwall boundary layers were confined to about $30 \%$ of span from both endwalls, thereby yielding a region of uniform total pressure around midspan $(0.3<z / h<0.7)$. In all cases, the axial velocity density ratio was measured to be very close to 1.0 , which is a necessary condition for acceptable two-dimensionality of the profile flow. Despite differences in the inlet and outlet flow angles, all airfoils were designed to give similar convergence ratios $(C R)$ at their design operating point.

The high flow turning airfoils have larger Mach number and row velocity ratios than the low-turning airfoils. The airfoils may also be split into two additional categories based on the Zweifel coefficients: the "baseline loaded" SL1 and SL3 are representative of some current HP stage blade designs, and the "more highly loaded" SL2, SL2P and SL4 have a $30 \%$ increase in loading due to increased spacing. Finally, the relatively high outlet Reynolds numbers in this experiment are expected to reduced the effects of 
temperature-related $R e$ variations in the test section (e.g. total temperature drop during the wind tunnel run).

Table 6.1 Summary of cascade parameters at the design point $\left(M_{2, i s}=0.8\right)$

\begin{tabular}{|c|c|c|c|c|c|}
\hline & SL1F & SL2F & SL2P & SL3F & SL4F \\
\hline Inlet Turbulence Intensity, $T u_{i n}$ & \multicolumn{5}{|c|}{$4 \%$} \\
\hline Inlet Turbulence Length Scale, $L_{t, i n}$ & \multicolumn{5}{|c|}{$0.6 \mathrm{C}_{\mathrm{X}}$} \\
\hline Inlet Mach Number, $M_{1}$ & 0.286 & 0.283 & 0.294 & 0.305 & 0.302 \\
\hline Outlet Isentropic Mach Number, $M_{2, i s}$ & 0.78 & 0.78 & 0.78 & 0.78 & 0.78 \\
\hline Outlet Mach Number, $M_{2}$ & 0.76 & 0.76 & 0.77 & 0.76 & 0.77 \\
\hline Mach Number Ratio $\left(M_{2} / M_{1}\right), M R$ & 2.66 & 2.69 & 2.62 & 2.49 & 2.55 \\
\hline Row Velocity Ratio $\left(V_{2} / V_{1}\right), R V R$ & 2.54 & 2.56 & 2.50 & 2.38 & 2.43 \\
\hline Inlet Flow Angle, $\beta_{1}\left(^{\circ}\right)$ & 43.5 & 43.5 & 43.5 & 27.0 & 27.0 \\
\hline Outlet Flow Angle, $\beta_{2}\left({ }^{\circ}\right)$ & 69.1 & 68.2 & 68.5 & 62.9 & 63.0 \\
\hline Total Flow Turning, $\theta\left(^{\circ}\right)$ & 112.6 & 111.7 & 112.0 & 89.9 & 90.0 \\
\hline Convergence Ratio $\left(\cos \beta_{1} / \cos \beta_{2}\right), C R$ & 2.03 & 1.95 & 1.98 & 1.96 & 1.96 \\
\hline Axial Velocity Ratio, $A V R$ & 1.25 & 1.31 & 1.26 & 1.22 & 1.24 \\
\hline Axial Velocity Density Ratio, $A V D R$ & 0.98 & 1.02 & 0.99 & 0.98 & 0.98 \\
\hline Inlet Reynolds Number, $R e_{C, 1}$ & 270,000 & 280,000 & 280,000 & 290,000 & 300,000 \\
\hline Outlet Reynolds Number, $R e_{C, 2}$ & 580,000 & 600,000 & 590,000 & 580,000 & 620,000 \\
\hline Zweifel Coefficient, $Z w$ & 0.75 & 0.97 & 0.99 & 0.78 & 1.01 \\
\hline
\end{tabular}

The off-design Mach number investigations, summarized in Table 6.2, indicate several changes in the aerodynamic characteristics with Mach number: increasing the Mach number results in higher row velocity ratio and axial velocity ratio. The total flow turning and the convergence ratios also increase due to slightly larger outlet flow angles (reduced deviation). The axial velocity density ratio, on the other hand, remains close to 1.0, as expected for two-dimensional flows. Although both inlet and outlet Reynolds numbers are affected by the operating point, the lowest value of $\operatorname{Re}_{C, 2}$ still remains relatively high at approximately 480,000. An interesting feature in Table 6.2 is the drop in the Zweifel coefficient at higher Mach numbers due to the larger available "reference loading" $\left(P_{01}-P_{S 2}\right)$ compared to the actual loading. Similarly, the total pressure losses are non-dimensionalized by the dynamic pressure at the outlet of the cascade $\left(P_{02}-P_{S 2}\right)$, which shows a similar increase with Mach number. 
Table 6.2 Summary of cascade parameters at off-design Mach numbers

\begin{tabular}{|c|c|c|c|c|c|c|}
\hline & \multicolumn{2}{|c|}{ SL1F } & \multicolumn{2}{|c|}{ SL2F } & \multicolumn{2}{|c|}{ SL2P } \\
\hline Inlet Turbulence Intensity, $T u_{\text {in }}$ & \multicolumn{6}{|c|}{$4 \%$} \\
\hline Inlet Turbulence Length Scale, $L_{t, i n}$ & \multicolumn{6}{|c|}{$0.6 \mathrm{C}_{\mathrm{X}}$} \\
\hline Inlet Mach Number, $M_{1}$ & 0.265 & 0.294 & 0.263 & 0.291 & 0.273 & 0.300 \\
\hline Outlet Isentropic Mach Number, $M_{2, i s}$ & 0.65 & 0.91 & 0.65 & 0.91 & 0.65 & 0.90 \\
\hline Outlet Mach Number, $M_{2}$ & 0.64 & 0.87 & 0.64 & 0.89 & 0.64 & 0.88 \\
\hline Mach Number Ratio $\left(M_{2} / M_{1}\right), M R$ & 2.42 & 2.96 & 2.43 & 3.06 & 2.34 & 2.93 \\
\hline Row Velocity Ratio $\left(V_{2} / V_{1}\right), R V R$ & 2.34 & 2.78 & 2.36 & 2.87 & 2.27 & 2.75 \\
\hline Inlet Flow Angle, $\beta_{1}\left(^{\circ}\right)$ & 43.5 & 43.5 & 43.5 & 43.5 & 43.5 & 43.5 \\
\hline Outlet Flow Angle, $\beta_{2}\left(^{\circ}\right)$ & 68.9 & 69.3 & 67.9 & 68.6 & 68.1 & 68.9 \\
\hline Total Flow Turning, $\theta\left(^{\circ}\right)$ & 112.4 & 112.8 & 111.4 & 112.1 & 111.6 & 112.4 \\
\hline Convergence Ratio $\left(\cos \beta_{1} / \cos \beta_{2}\right), C R$ & 2.01 & 2.05 & 1.93 & 1.99 & 1.94 & 2.01 \\
\hline Axial Velocity Ratio, $A V R$ & 1.16 & 1.36 & 1.22 & 1.44 & 1.17 & 1.37 \\
\hline Axial Velocity Density Ratio, $A V D R$ & 0.98 & 0.99 & 1.02 & 1.02 & 0.98 & 0.98 \\
\hline Inlet Reynolds Number, $R e_{C, 1}$ & 230,000 & 310,000 & 240,000 & 310,000 & 240,000 & 310,000 \\
\hline Outlet Reynolds Number, $R e_{C, 2}$ & 480,000 & 680,000 & 500,000 & 690,000 & 490,000 & 670,000 \\
\hline Zweifel Coefficient, $Z w$ & 0.81 & 0.66 & 1.07 & 0.89 & 1.07 & 0.90 \\
\hline
\end{tabular}

\subsection{Overview of Cases Examined}

The completed experimental and numerical investigations are summarized below. As indicated in Table 6.3, the experimental investigations were performed at three different exit Mach numbers. Additional computations were performed to try to separate the effects of Mach number and Reynolds number variations on profiles losses, as indicated in Table 6.4 and Table 6.5.

Table 6.3 Summary of the experimental and computational investigations

\begin{tabular}{|c|c|c|c|c|c|c|c|c|c|c|c|}
\hline & \multicolumn{3}{|c|}{ SL1 } & \multicolumn{3}{c|}{ SL2 } & \multicolumn{3}{c|}{ SL2P } & SL3 & SL4 \\
\hline$M_{2, \text { is }}$ & 0.65 & 0.78 & 0.91 & 0.65 & 0.78 & 0.91 & 0.65 & 0.78 & 0.90 & 0.78 & 0.78 \\
\hline $\operatorname{Re}_{C, 2}\left(\times 10^{-3}\right)$ & 480 & 580 & 680 & 500 & 600 & 690 & 490 & 590 & 670 & 580 & 620 \\
\hline
\end{tabular}

Table 6.4 SL2 computational investigations: Mach number variation at constant Reynolds number $\left(R e_{C, 2}=600,000\right)$

\begin{tabular}{|l|l|l|l|l|l|l|}
\hline $\boldsymbol{M}_{2, \boldsymbol{i} \text { is }}$ & 0.60 & 0.70 & 0.80 & 0.90 & 0.95 & 1.00 \\
\hline
\end{tabular}


Table 6.5 SL2 computational investigations: Reynolds number variation at constant Mach number $\left(M_{2, i s}=0.8\right)$

\begin{tabular}{|l|l|l|l|l|l|}
\hline $\boldsymbol{R} \boldsymbol{e}_{\boldsymbol{C}, \boldsymbol{2}}$ & 400,000 & 500,000 & 600,000 & 700,000 & 800,000 \\
\hline
\end{tabular}

\subsection{Airfoil Loading Distributions}

\subsubsection{Experimental and Numerical Loading Results at the Design Mach Number}

The experimental results include inlet and outlet total and static pressures, and bladesurface static pressures. The inlet values correspond to the centreline free-stream flow and the outlet values are fully mixed-out. Due to the limited number of available surface static taps in the experiments, extensive use has been made of the blade loading distributions predicted by the CFD.

The measured and computed distributions of midspan surface isentropic Mach numbers are presented in Figure 6.1 and Figure 6.2. The surface Mach numbers are plotted as a function of axial distance $\left(x / C_{X}\right)$ on the left and normalized surface length $\left(S / S_{\max }\right)$ on the right. Use of the latter has the advantage of showing more clearly the streamwise pressure gradients experienced by the surface boundary layers. The results also illustrate the close agreement between the CFD predictions and the experimental measurements.

The isentropic surface Mach numbers, defined earlier, are slightly larger than the actual Mach numbers since the total pressure was not corrected for any losses. This effect, however, is expected to be small due to the relatively low Mach numbers (e.g. normal shock loss at Mach 1.1: $\left.\Delta P_{0} / P_{01}=0.001\right)$.

In Figure 6.1, the rise in surface Mach numbers around the leading edge $\left(0<S / S_{\max }<\right.$ 0.05) results in strong favourable pressure gradients on both suction and pressure surfaces. On the suction surface, the favourable pressure gradients extend from the leading edge to the suction peak, apart from a weak inflection point at approximately 0.07 $S / S_{\max }$. The inflection point, which may cause flow separation, is associated with the change in airfoil curvature as the leading edge ellipse connects to the suction surface profile. Downstream of the suction peak, SL2 displays stronger adverse pressure gradients than SL1. Part of the pressure surface for SL1 is also exposed to adverse pressure gradients $\left(0.05<S / S_{\max }<0.25\right)$. Consequently, the CFD predicts a short 
pressure-surface separation bubble on SL1 that will be evident in the plots of skin friction coefficient. The pressure-surface (PS) boundary layers are then accelerated smoothly towards the trailing edge $\left(0.3<S / S_{\max }<1.0\right)$ to achieve the required outlet Mach number.
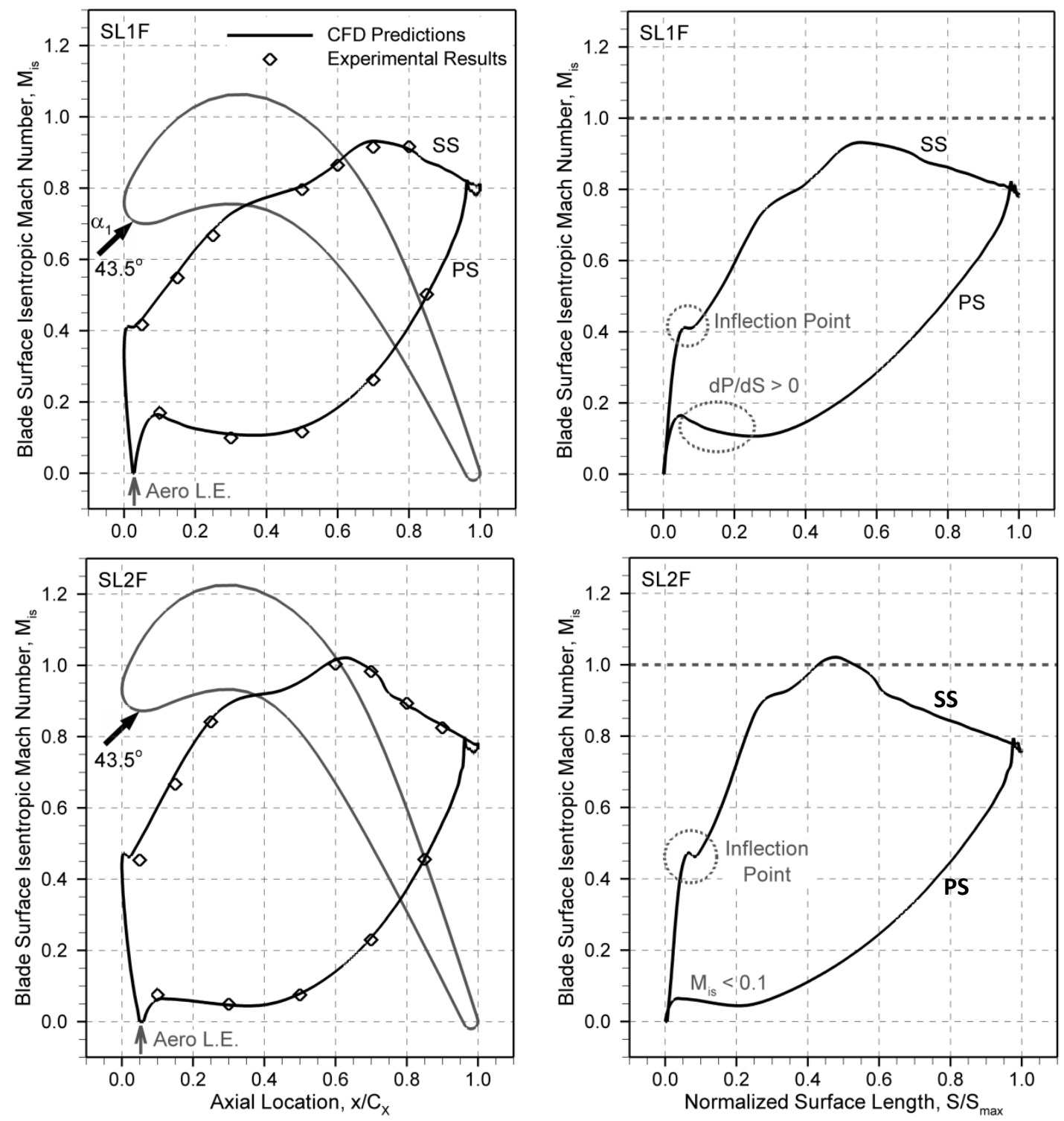

Figure 6.1 High turning airfoils: blade surface Mach numbers versus axial chord location (left) and normalized surface length (right) at the design Mach number $\left(M_{2, i s}=0.8\right)$

The SL3 loading distribution in Figure 6.2 does not display adverse pressure gradients on the pressure surface. Furthermore, the controlled diffusion on the suction surface reduces the possibility of suction-surface (SS) flow separation. SL4, on the other 
hand, displays larger adverse pressure gradients on the suction surface, accompanied with a region of supersonic flow around the suction peak.
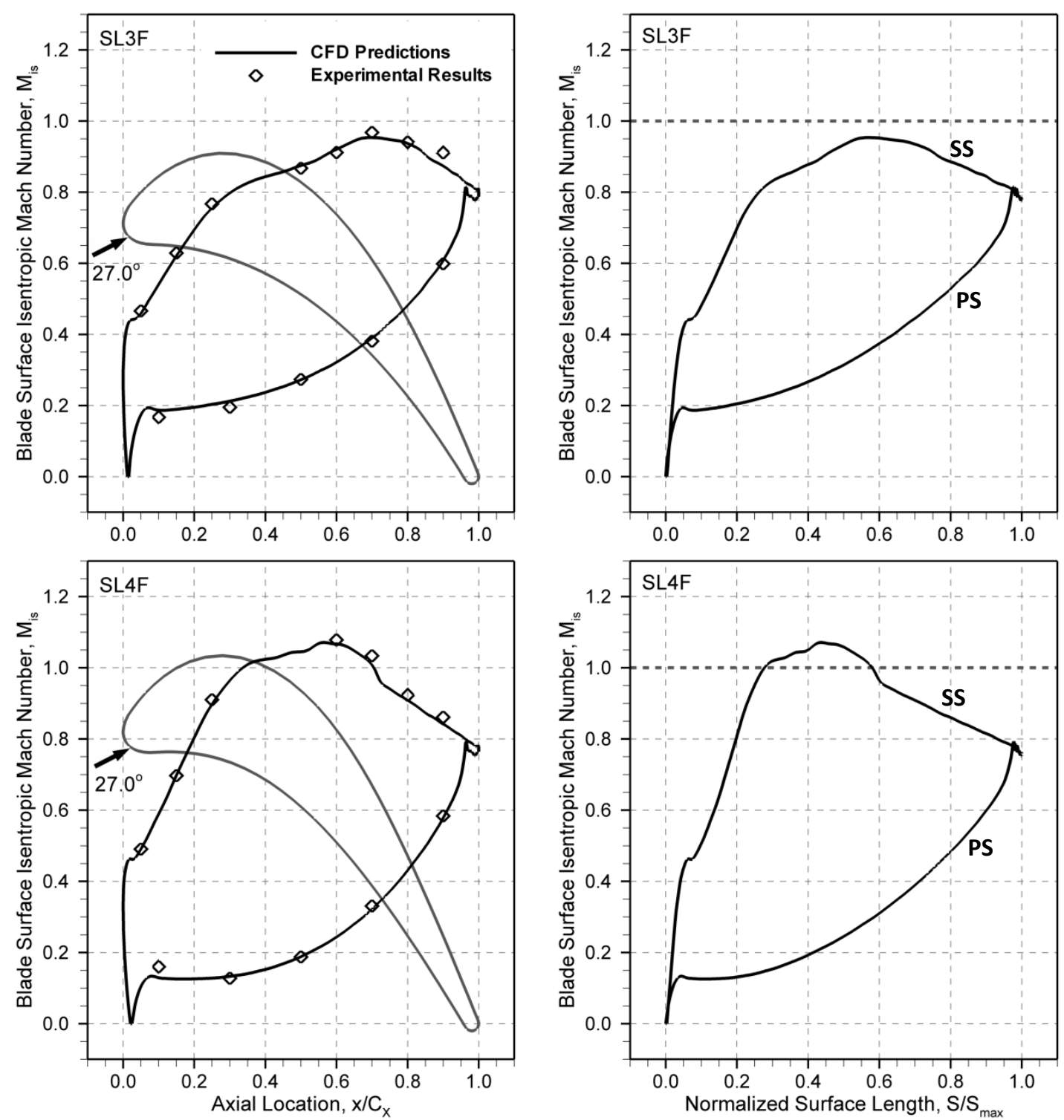

Figure 6.2 Low turning airfoils: blade surface Mach numbers versus axial chord location (left) and normalized surface length (right) at the design Mach number $\left(M_{2, i s}=0.8\right)$

In terms of the overall loading levels, SL2 and SL4 $\left(Z w_{\text {des }} \approx 1.0\right)$ are clearly more highly loaded than SL1 and SL3 ( $\left.Z w_{\text {des }} \approx 0.8\right)$. The additional loading is in part realized through lower SS static pressures, and thus higher local velocities (e.g. supersonic flow at $0.5 \mathrm{~S} / \mathrm{S}_{\max }$ ). As shown by Denton (1993), the entropy generation within an attached boundary layer is proportional to the cube of the edge velocity. Therefore, in the absence of any separation bubbles, the more highly-loaded airfoils are anticipated to generate 
larger profile losses than the baseline-loaded airfoils. The losses are examined in Section 6.5.1.

As described in Chapter 3, an additional highly-loaded airfoil, designated SL2P, was also investigated. SL2P has the same overall geometric and aerodynamic parameters as SL2. However, the loading distribution has been modified with the goal of reducing the secondary losses. The effects of the new loading distribution on the secondary flow characteristics are discussed in Chapter 9.

The midspan loading distribution for SL2P is shown in Figure 6.3, and compared with the SL2 loading distribution in Figure 6.4. SL2P displays higher PS Mach numbers than SL2 in the forward part of the passage. On the suction surface, SL2P displays a nearly-smooth, favourable pressure gradient, extending from the leading edge to the suction peak. In comparison, the SL2 distribution indicates a weak inflection point at about $0.07 \mathrm{~S} / \mathrm{S}_{\max }$. The SS Mach number distributions on SL2 and SL2P display similar surface Mach numbers in the aft part of the passage $\left(0.7<x / C_{X}<1.0\right)$.
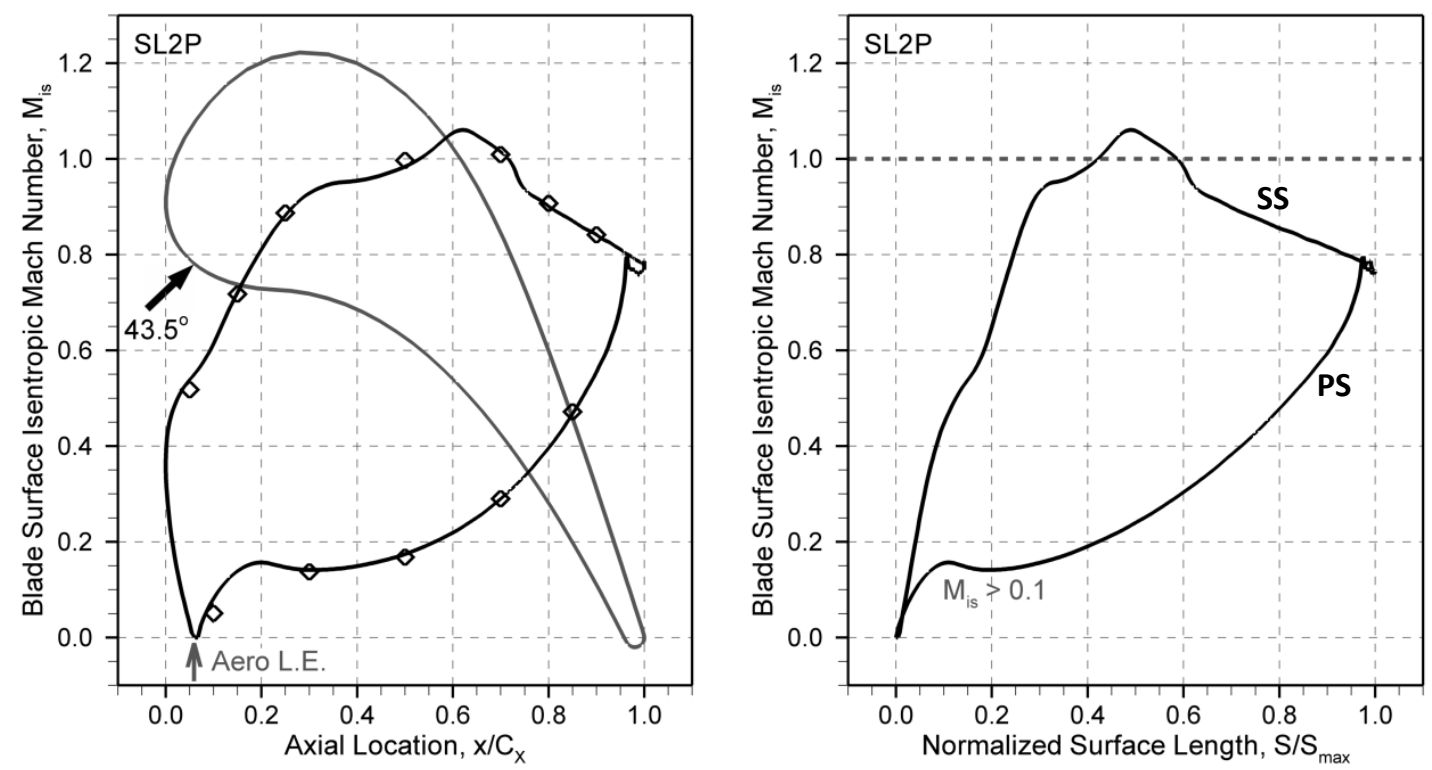

Figure 6.3 Pressure-side modified airfoil: blade surface Mach numbers versus axial chord location (left) and normalized surface length (right) at the design Mach number $\left(M_{2, i s}=0.8\right)$

The loading distributions are co-plotted in Figure 6.4 for direct comparisons: SL1 and SL3 are considered aft-loaded, whereas SL2 and SL4, which display larger cross-passage pressure differences near the leading edge, are more mid-loaded. The mid- to aft-loading 
characteristics of these airfoils are typically expected to reduce the secondary losses (Zoric, 2006; Knezevici, 2011). Of course, the exact position of the suction peak is also influenced by the required diffusion on the suction surface. SL1 and SL3 display smaller SS Mach numbers than SL2 and SL4, which are more highly-loaded; therefore, it is possible to shift the suction peak locations further aft for SL1 and SL3.
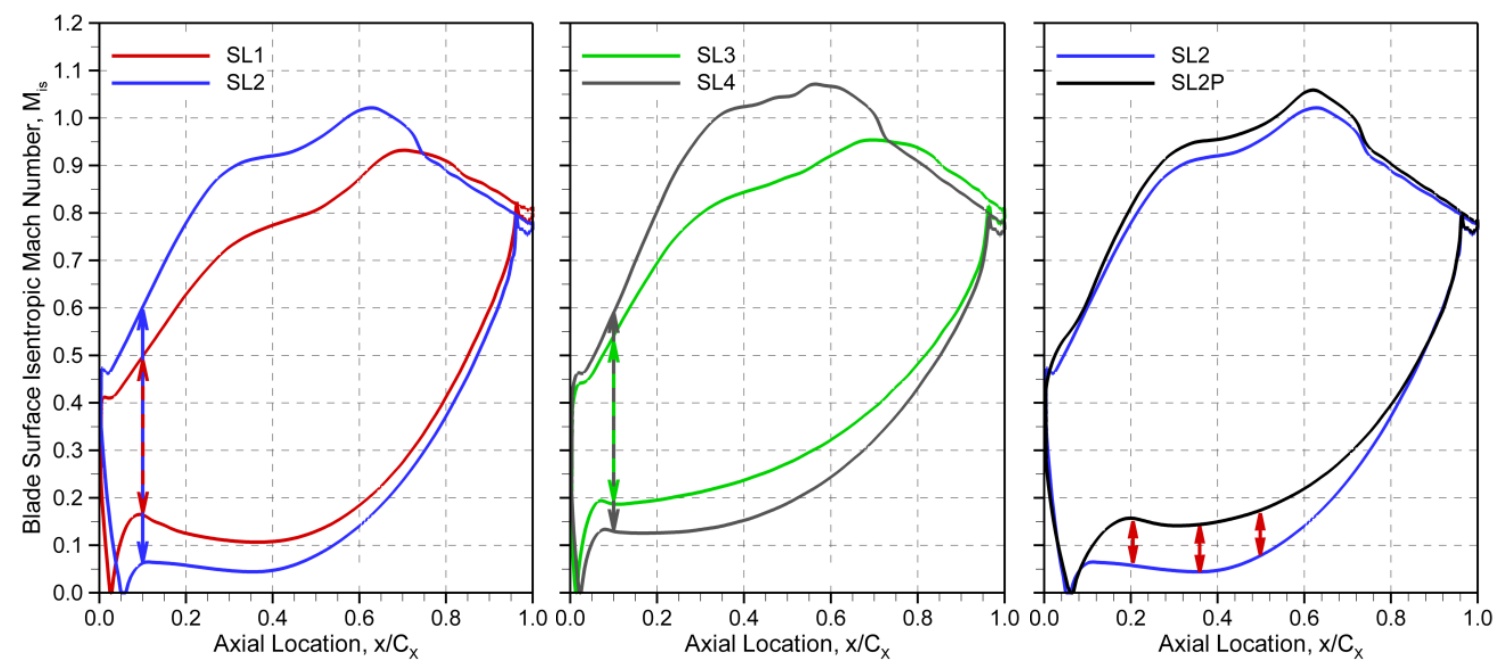

Figure 6.4 Blade surface Mach numbers at the design Mach number $\left(M_{2, i s}=0.8\right)$

The non-dimensional surface pressure gradients on the pressure surfaces are shown in Figure 6.5 in the form of the acceleration parameter $(A C)$. Here, negative and positive acceleration parameters correspond to adverse and favourable pressure gradients, respectively.

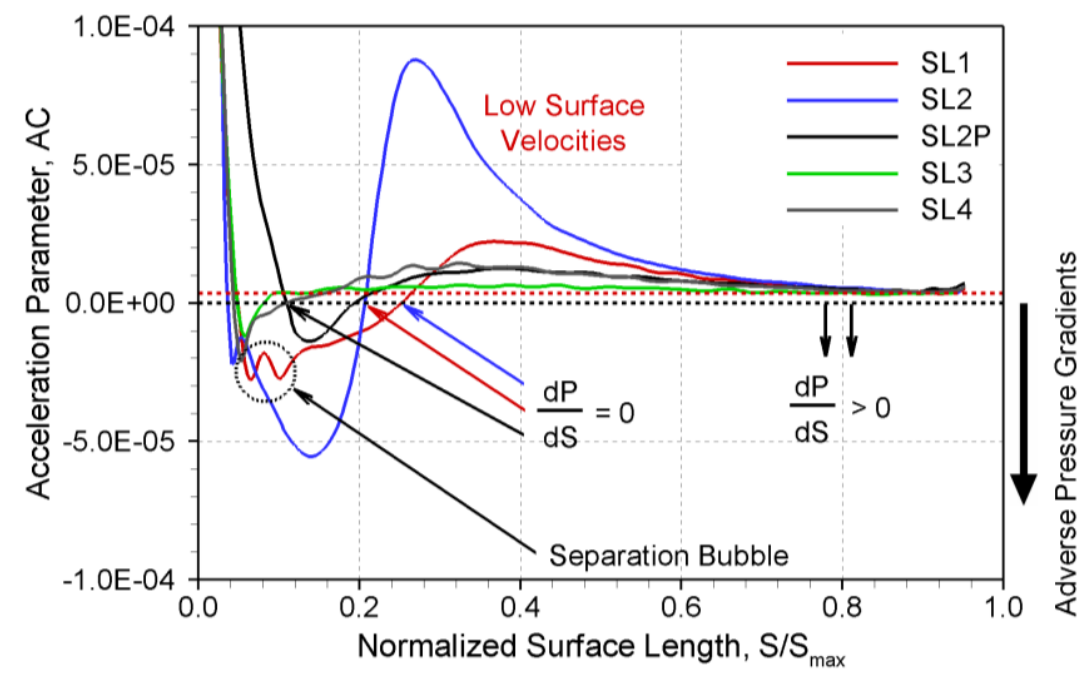

Figure 6.5 Pressure surface acceleration parameters at the design Mach number 
In Figure 6.1 and Figure 6.2, the PS loading distributions display generally mild pressure gradients from about $5 \%$ to $95 \%$ surface lengths. The acceleration parameters in Figure 6.5 , on the other hand, show larger variations due to the relatively small local velocities. The critical value of the acceleration parameter for flow relaminarization $\left(A C_{c r i t}=3.5 \times 10^{-6}\right)$ is shown using a red dashed line in Figure 6.5. It should be clarified that once transition has occurred, turbulence fluctuation continues in the boundary layer. However, under strong acceleration, the dissipation rate (i.e. $v(\partial u / \partial y)^{2}$ terms) becomes high and reduces the turbulence intensity to low levels, creating quasi-laminar flow. Positive acceleration parameters, larger than $A C_{\text {crit }}$, are generated just downstream the leading edge $\left(0<S / S_{\max }<0.05\right)$ as the flow accelerates away from the stagnation point. This is followed by adverse pressure gradients extending over a considerable portion of the pressure surface on SL1 $\left(0.05<S / S_{\max }<0.25\right)$, which result in a small laminar separation bubble according to the CFD predictions. Following the turbulent reattachment, the PS boundary layer on SL1 is once again subjected to favourable pressure gradients $\left(S / S_{\max }>0.25\right)$ that may result in relaminarization by approximately $0.6 S / S_{\max }$.

The variations in acceleration parameter are most pronounced on SL2 mainly due to the low PS Mach numbers in the forward part of the passage (Figure 6.1). The acceleration parameters on SL2P are smaller than those on SL2 due to the larger free stream velocities.

The suction-surface acceleration parameters are shown in Figure 6.6. Overall, the suction surfaces display smaller acceleration parameters than the pressure surfaces due to higher free-stream velocities. The suction surface characteristics are very similar for all cases, apart from differences in the location of the suction peaks. The acceleration parameters are very large near the leading edge as flow accelerates away from the stagnation point. In each case, the acceleration parameter rises again beyond the critical value after the inflection point, thereby potentially retaining the laminar boundary layer towards the suction peak.

The presence of separation bubbles were examined both numerically based on the computed surface shear stresses, and experimentally using the flow visualization results. 
The predicted skin friction coefficients at the design Mach number are presented in Figure 6.7.

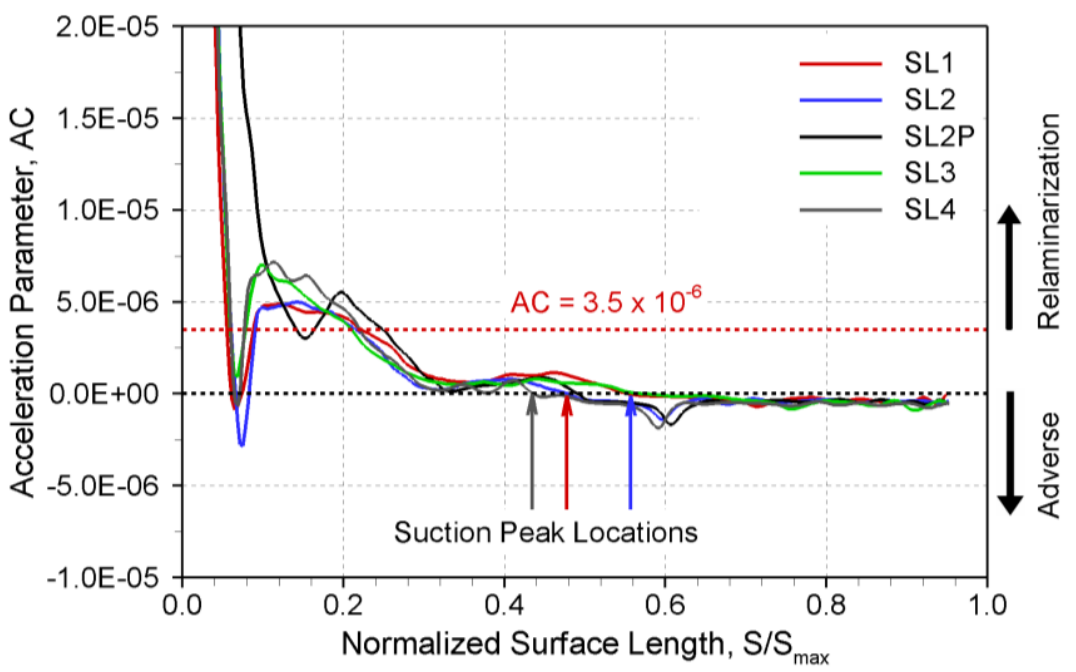

Figure 6.6 Suction surface acceleration parameters at the design Mach number
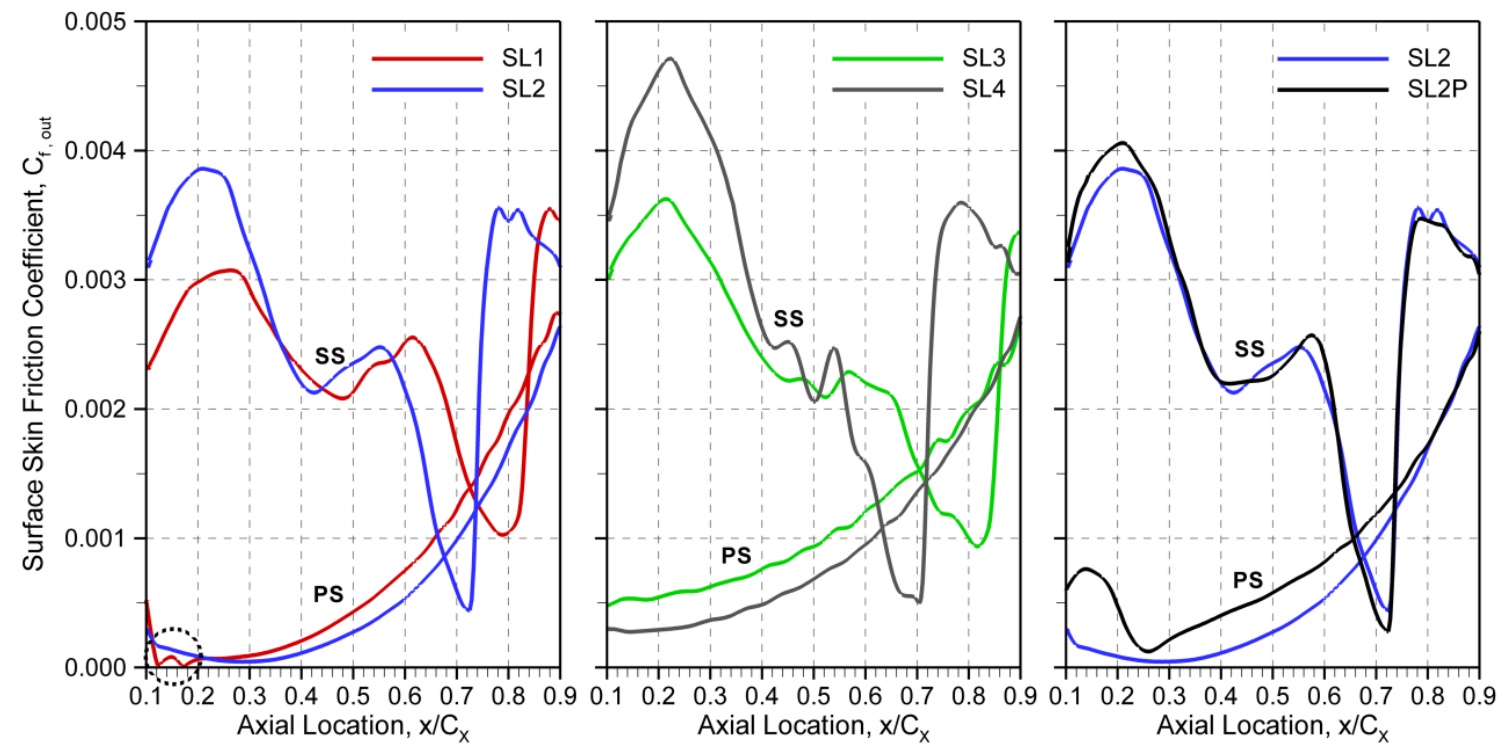

Figure 6.7 Blade surface skin friction coefficients from $\operatorname{CFD}\left(M_{2, i s}=0.8\right)$

Overall, the skin friction coefficients are larger on the suction surfaces, extending from the leading edge to the suction peak, than on the pressure surfaces. The surface shear stresses and thus the skin friction coefficients decrease in the presence of adverse pressure gradients (e.g. past the suction peaks). Boundary layer separation is only apparent on the SL1 pressure surface (for $0.12<x / C_{X}<0.18$ ) at the design Mach number. The more highly loaded cascades, SL2 and SL4, display larger values of $C_{f}$ on the suction 
surface in the forward part of the passage than SL1 and SL3. On the other hand, SL1 and SL3 retain larger skin friction coefficients past the suction peaks due to smaller adverse pressure gradients. The predicted skin friction coefficients rise abruptly on the aft part of the suction surfaces as a result of transition to turbulence (e.g. at $0.75 x / C_{X}$ on SL2). The design goal of limiting the adverse pressure gradients to avoid boundary layer separation seems to have been met for all cases.

In addition to the computations, experimental flow visualization studies were performed for SL1, SL2 and SL2P at the design Mach number. The surface flow visualization results around the midspan sections $(0.4<z / h<0.6)$ are presented in Figure 6.8. Flow separation and reattachment are shown using dashed lines and dotted lines, respectively.
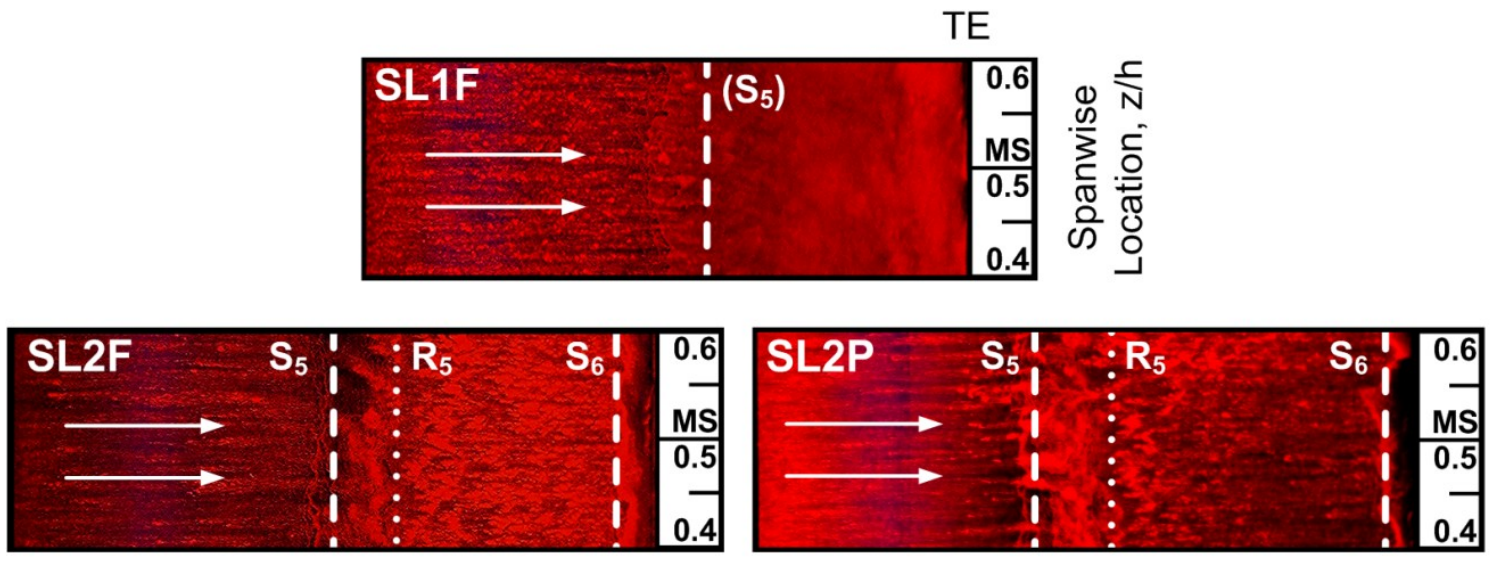

Figure 6.8 Midspan surface flow visualization results at the design Mach number

The flow visualization results display evidence of a small SS separation bubbles (with separation at $\mathrm{S}_{5}$ ), which are not predicted by the transitional CFD simulations. These findings were also supported by the videos recorded during the wind tunnel runs, which clearly document the formation of the separation bubbles on the blade surfaces.

In Figure 6.8, the separated profile flow on SL1 does not clearly reattach on the suction surface, indicating a region of very low surface shear stress. However, measured profile losses for SL1, presented in Section 6.5.1, are not typical of those commonly associated with a stalled airfoil. Two hypotheses are put forth to explain the observed discrepancies: the relatively low-stress, near-surface, shear layer either remains close to the blade surface downstream of $\mathrm{S}_{5}$ due to high local Mach numbers and low acceleration 
parameters (relatively weak adverse pressure gradients), or it reattaches briefly prior to subsequent separation at the trailing edge, similar to separation line $\mathrm{S}_{6}$ on SL2. Although it is not possible to determine the precise state of the suction-surface boundary layer on SL1 downstream of $\mathrm{S}_{5}$ in Figure 6.8, the ensuing increase in profile losses is nonetheless measurable as will be seen later.

The SL2 and SL2P airfoils, both utilizing the same suction surface profile, also show evidence of SS flow separation $\left(\mathrm{S}_{5}\right)$. However, in contrast to SL1, the flow clearly reattaches $\left(\right.$ at $R_{5}$ ) in these cases, resulting in short separation bubbles and perhaps separated-flow transition (in contrast, CFD predicts attached boundary layers). In each case, additional flow separation is identified near the trailing edge (at $\mathrm{S}_{6}$ ) due to the sudden expansion in this region; this was particularly evident in the flow visualization videos.

\subsubsection{Experimental and Numerical Loading Results at Off-Design Mach Numbers}

As noted in Table 6.2, the effects of varying the outlet Mach number were examined experimentally for SL1, SL2 and SL2P. The results for the loading distributions are presented in Figure 6.9.

Increasing the outlet Mach number mainly influences the suction surface behaviour, particularly in the aft part of the passage: the airfoil surface is exposed to a short length of supersonic flow at the highest outlet Mach number. The surface Mach numbers in the forward part of the passage $\left(x / C_{X}<0.5\right)$, on the other hand, vary mainly with inlet Mach number until the flow reaches the choke point.

Increasing the exit Mach number results in more aft loading and larger SS adverse pressure gradients. At the highest Mach number, the region of lowest pressure extends over a larger portion of the suction surface towards the shock impingement location. On SL1, the computations displayed fully-attached SS boundary layers at all examined Mach numbers. On SL2 and SL2P, the suction peak is terminated by a shock wave at the highest Mach number, resulting in a large static pressure rise at $0.8 x / C_{X}$. Consequently, a small SS separation bubble is predicted by the CFD on SL2 and SL2P at the highest Mach number. The off-design behaviour is discussed further in the next section based on additional numerical simulations for SL2. 

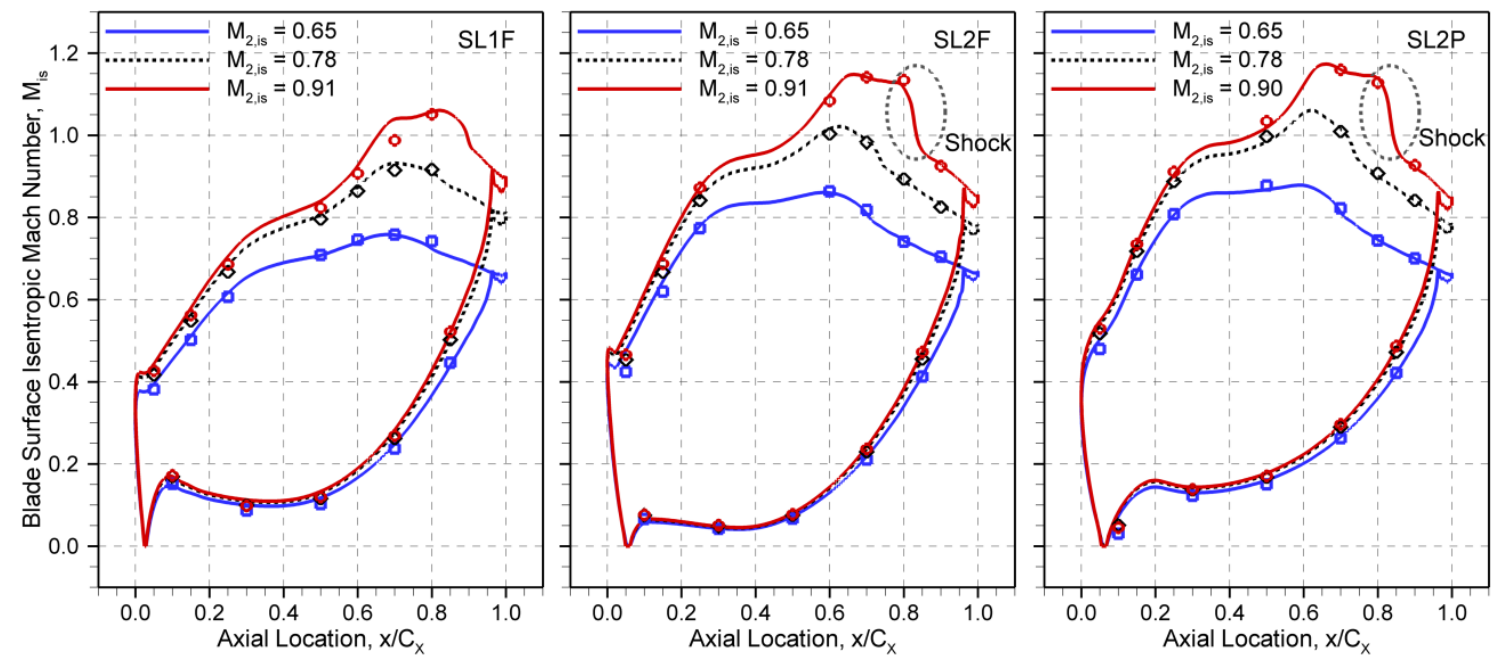

Figure 6.9 Blade surface Mach number distributions at off-design Mach numbers

\subsubsection{Additional Effects of Mach Number and Reynolds Number on Loadings: CFD Results}

Further parametric CFD studies were conducted for SL2 to characterize the loading distributions at off-design Mach numbers and Reynolds numbers. The effects of offdesign Mach numbers are first examined in order to relate the loading distribution to the Zweifel coefficient. In these studies, the Reynolds number was kept constant at 600,000 in contrast to the variations present in the experiments (Table 6.2). The surface Mach number distributions and the skin friction coefficients are shown in Figure 6.10.

As previously discussed, increasing the outlet Mach number results in larger SS Mach numbers and increased aft loading. The resulting SS adverse pressure gradients cause boundary layer separation at Mach numbers above 0.9 , which appear as small separation bubbles in the $C_{f}$ plot. The overall reduction in skin friction coefficient with Mach number is mainly attributed to the corresponding increase in outlet dynamic pressure (i.e. the non-dimensionalizing parameter). In other words, although the surface shear stresses increase slightly with Mach number due to smaller boundary layer shape factors (not shown here), the skin friction coefficients decrease due to significantly larger outlet dynamic pressures. As the outlet Mach number is raised from 0.9 to 1.0, the shape of the suction peak changes markedly: at Mach 1.0, the suction surface is exposed to supersonic flow from $0.5 x / C_{X}$ to $0.95 x / C_{X}$. In this region, the skin friction coefficients decrease around the first peak $\left(0.6<x / C_{X}<0.7\right)$, but then recover towards the second peak 
(expansion waves), followed by shock impingement and flow separation near the trailing edge $\left(0.9<x / C_{X}<0.95\right)$.
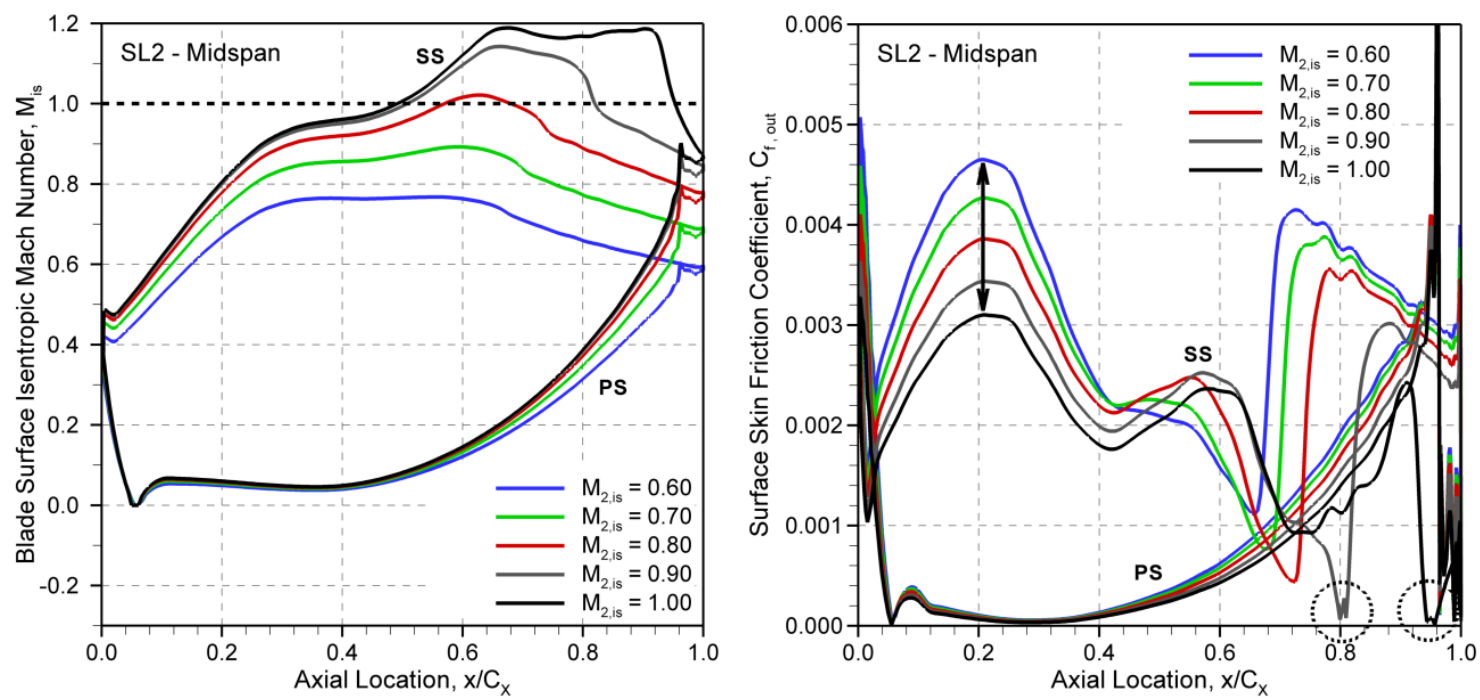

Figure 6.10 Mach number variation in SL2: surface Mach numbers and skin friction coefficients

A new blade loading parameter, $C_{P S, Z w}$, is defined here to compare the loading distributions with the Zweifel coefficients. The new parameter is defined based on the pseudo-dynamic pressure " $P_{01}-P_{S 2}$ ", which is consistent with the definition of the Zweifel coefficient in Chapter 4:

$$
C_{P S, Z w}=\frac{P_{S, T E}-P_{S}}{P_{01}-P_{S 2}}
$$

where $P_{S, T E}$ refers to the static pressure at the trailing edge (to facilitate the comparisons at different exit Mach numbers).

The loading distributions in terms of $C_{P S, Z w}$, and the overall loading levels in terms of $Z w$ (Equation 4.12) and $Z w_{\text {Incompressible }}$ (Equation 4.13) are presented in Figure 6.11. The integrated values of $C_{P S, Z w}$ in Figure 6.11, therefore, correspond to the original definition of the Zweifel coefficient (Zweifel, 1939). The outlet flow angles, also shown in Figure 6.11, increase only slightly with Mach number (less than $0.5^{\circ}$ ), and therefore do not affect the Zweifel coefficients significantly. It is evident that increasing the Mach number reduces the overall loading level in both plots as the "reference loading" increases at a faster rate than the actual loading. The incompressible definition of the Zweifel coefficient, which utilizes $1 / 2 \rho V^{2}$ as the non-dimensionalizing parameter instead of " $P_{01}$ - 
$P_{S 2}$,", also shows a decreasing trend, although inconsistently with the original definition $\left(\Delta Z w_{\text {incompressible }}<\Delta Z w\right)$.
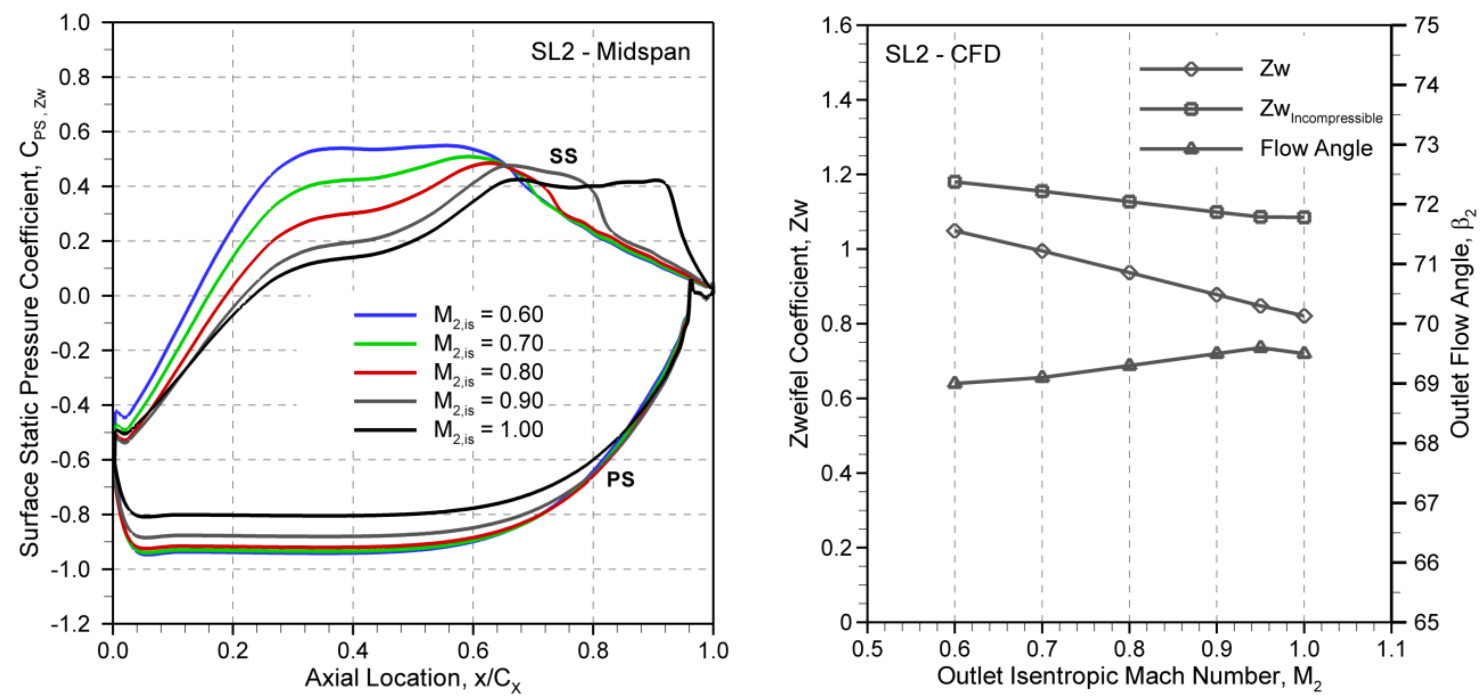

Figure 6.11 Mach number variation in SL2: $C_{P S, Z w}, Z w$ and $\beta_{2}$ distributions

The effects of Reynolds number variation on blade loading distributions at the design Mach number $\left(M_{2, i s}=0.8\right)$ are shown in Figure 6.12. The surface Mach numbers appear essentially independent of Reynolds number for the range examined here. The small changes in surface Mach numbers around the suction peak are associated with the displacement thickness of the SS boundary layer: as the Reynolds number is reduced, both the boundary layer thickness and the displacement thickness increase, thereby effectively changing the aerodynamic shape of the airfoil and its pressure distribution. The skin friction coefficients, on the other hand, display a clear trend with $R e$ reduction: $C_{f}$ increases in the forward part of the suction surface and in the aft part of the pressure surface. Following the suction peak, the $C_{f}$ values initially remain lower for the smaller $R e$ cases $\left(0.7<x / C_{X}<0.8\right)$, and then rise again towards the trailing edge. Varying the Reynolds number is also anticipated to affect the predicted profile losses due to the observed changes in $C_{f}$, and this will be discussed in Section 6.5.2. 

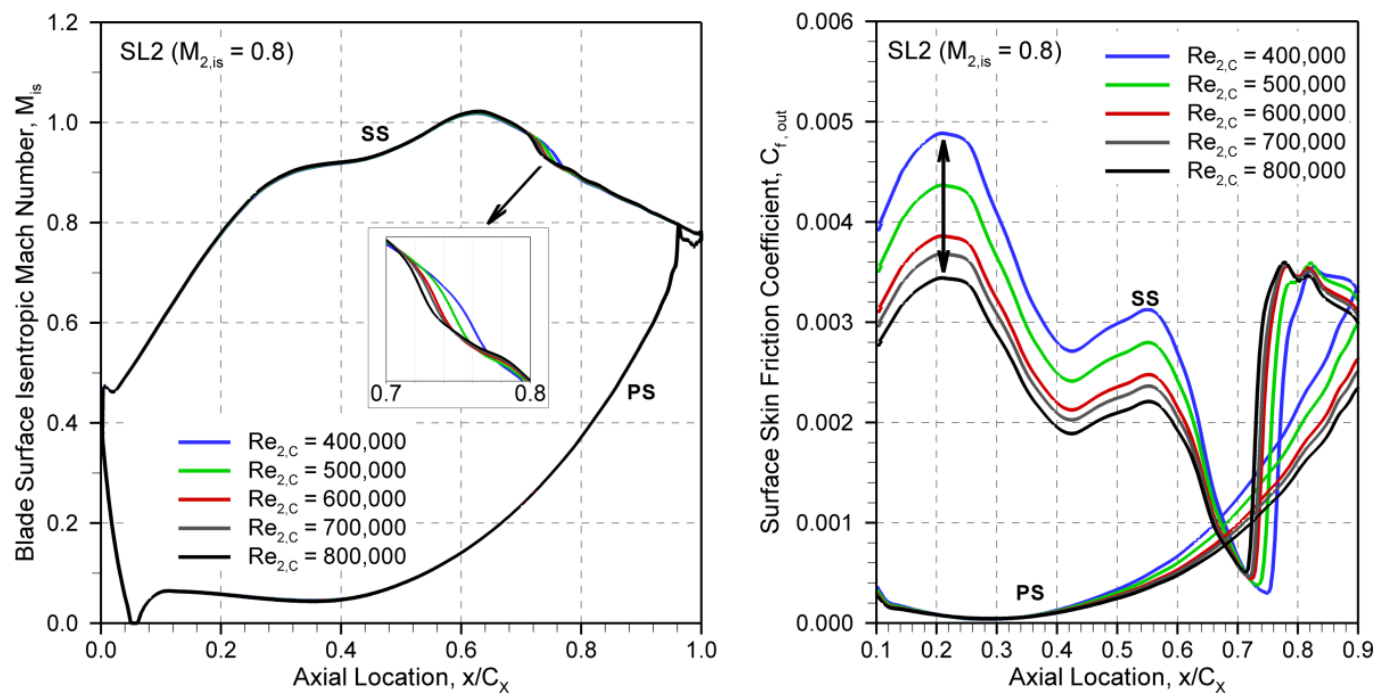

Figure 6.12 Reynolds number variation in SL2: $M_{i s}$ and $C_{f}$ distributions

\subsection{Profile Flows and Losses}

\subsubsection{Experimental and Numerical Results at Tested Mach Numbers}

As discussed, the experimental investigations were conducted at three different exit Mach numbers, and these were supplemented with CFD predictions at the corresponding operating points.

Profile losses in turbine cascades are affected by several factors to varying degrees depending on the flow regime:

- Viscous dissipation in attached boundary layers and in free shear layers due to flow separation (e.g. laminar separation bubbles)

- Entropy generation in the free-stream due to shock waves

- Shock wave / boundary layer interaction (e.g. turbulent separation bubbles)

- Trailing edge base pressure effects (Sieverding, 1980)

As discussed, the outlet Mach numbers are kept subsonic in the current project to minimize the influence of shock waves. Therefore, the discussions in this section are mainly focused on the effects of viscous dissipation in the boundary layers and the separation bubbles. The flow visualization results show evidence of small suctionsurface separation bubbles on SL1, SL2 and SL2P, whereas the CFD predictions only display pressure-surface separation on SL1. The downstream traverse results are used to 
quantify the effects of the separation bubbles on losses. The shock wave / boundary layer interaction effects are briefly examined in Section 6.5.2.

The profile flow measurements at the design Mach number are presented in Figure 6.13. The probe measurements have been collected at $1.4 C_{X}$, and as shown by Jouini (2000) the majority of the midspan wake mixing-out is typically completed by this traverse location (mixing of the blade surface boundary layers and the trailing edge shear layers). Additional downstream traverses at $1.7 C_{X}$ and $2.0 C_{X}$ confirmed this observation as the profile flow diffused without incurring much additional loss.
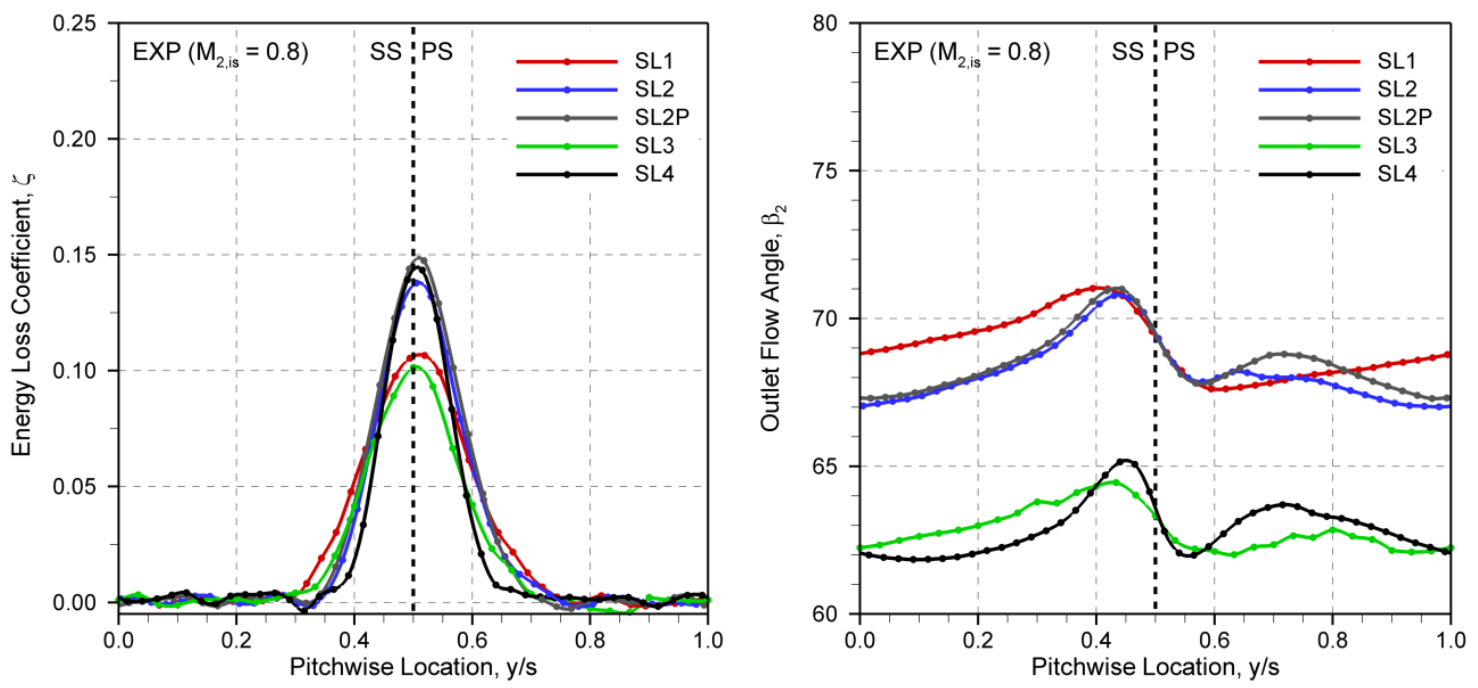

Figure 6.13 Profile flow measurements at the design Mach number at $1.4 C_{X}$ : energy loss coefficients (left) and exit flow angles (right)

Figure 6.13 shows distinct trends in flow angles and losses with the overall loading level: the flow angle distributions display similar overshoot and undershoot for the more highly loaded cascades (SL2, SL2P and SL4), and the wakes are slightly narrower with significantly higher peaks. SL1 displays the widest wake, thereby generating considerably higher profile losses than the similarly loaded SL3 $(Z w \approx 0.8)$. Although SL1 has higher flow turning than SL3, the additional losses are mainly attributed to the suction surface flow separation on SL1, as shown in Figure 6.8. In fact, SL1 produces similar profile losses to SL2, which is more highly loaded with similar flow turning. SL2 and SL2P generate wider wakes than SL4, which has less flow turning. SL2P displays a 
higher loss peak than SL2 at $0.5 y / s$, potentially as a result of the higher free-stream velocities on the pressure surface, as shown in Figure 6.4.

The predicted losses and flow angles at the design Mach number are presented in Figure 6.14. Compared to the experimental results, the CFD wakes are narrower with significantly higher peaks: the downstream mixing occurs more rapidly in the experiments than as predicted by CFD. SL1 displays the widest wake, analogous to the experiments, although with a slightly smaller peak than SL3. SL4, on the other hand, displays the narrowest wake with the highest peak at $0.5 \mathrm{y} / \mathrm{s}$. The angle distributions for the low-turning cascades also resemble the trends observed in the data, albeit with smaller under/overshoots. These trends are in good qualitative agreement with the experiments.
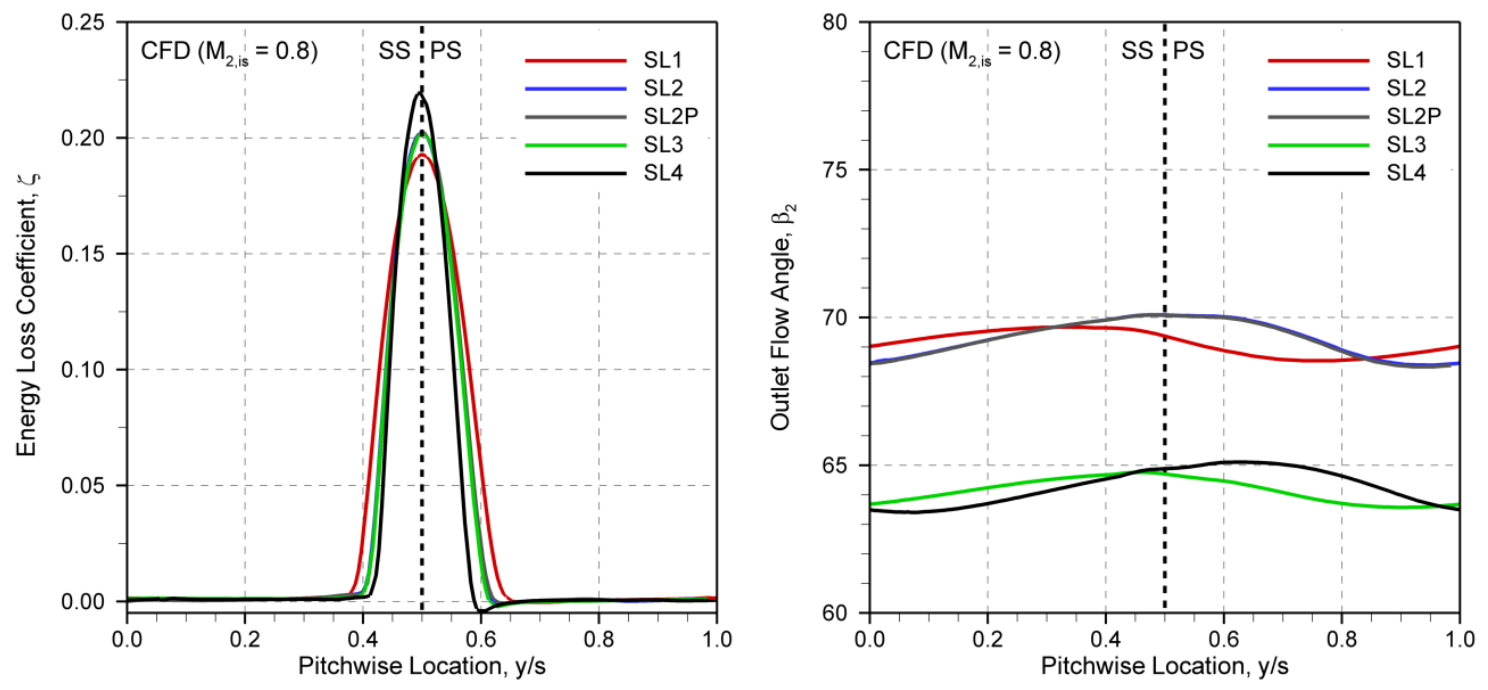

Figure 6.14 CFD predictions at the design Mach number at $1.4 C_{X}$ : energy loss coefficients (left) and exit flow angles (right)

The main discrepancy between the experiments and the CFD is identified for SL3: CFD predicts higher profile losses than expected. SL3 has low flow turning, and it is considered baseline loaded. The higher loss prediction is counterintuitive since SL3 display relatively low surface skin friction coefficients in Figure 6.7. In addition, the relatively small free-stream Mach numbers in Figure 6.2 suggest low viscous dissipation in the boundary layers, and there are no shock wave effects. Therefore, the additional losses are believed to be associated with trailing edge effects (Xu and Denton, 1988). 
As described in Chapter 4, additional losses are generated downstream of the measurement plane as the flow mixes out to fully uniform conditions. Unlike in secondary flows, the mixing of the profile flows does not typically introduce significant additional losses, particularly in the subsonic regime. Nevertheless, these losses are included here to eliminate any influence from the traverse plane location. The mixed out energy loss coefficients are presented in Figure 6.15. As indicated in Table 6.3, varying the exit Mach number also alters the Reynolds number in the experiments; therefore, the Reynolds numbers are also listed here.
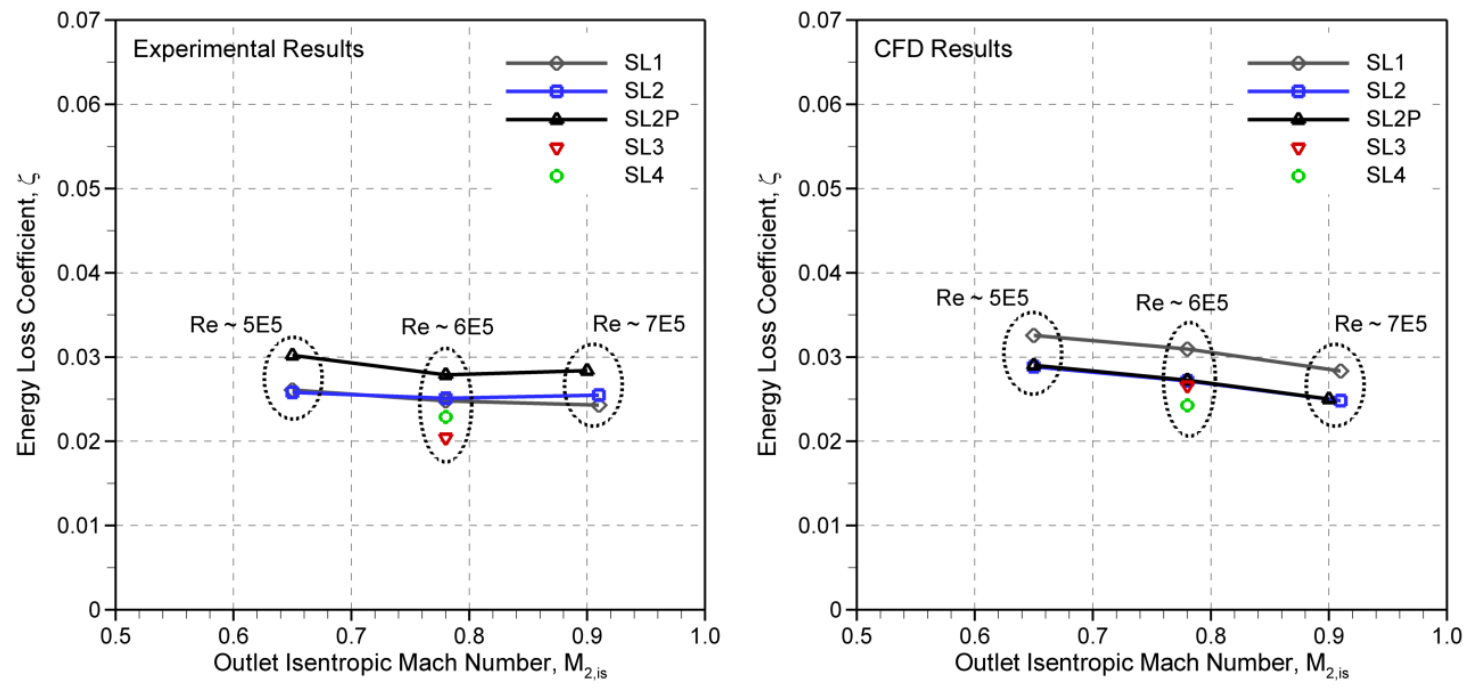

Figure 6.15 Mixed-out energy loss coefficients: experiments (left) and CFD (right)

Experimental results. Overall, the airfoils produce relatively low profile losses $(\zeta<$ 0.04), and the profile losses do not vary significantly over the range of operating points examined here. SL2P produces the highest losses at all investigated Mach numbers. SL1 and SL2 generate similar losses despite the additional loading for SL2, and this is partially attributed to the differences in the separation bubble characteristics in Figure 6.8. The low-turning cascades, in particular SL3, display smaller profile losses at the design Mach number than the high turning cascades.

CFD results. The CFD predictions do not fully corroborate the experimental trends. The predicted losses decrease slightly as the Mach number is raised; the effects of Mach number and Reynolds number variation on profile losses are investigated further in Section 6.5.2. SL1 generates the largest profile losses according to the computations, 
which may be associated with the predicted separation bubble on the pressure surface of SL1 in Figure 6.5. As in the experiments, SL4 generates lower profile losses than SL2 and SL2P according to the CFD, which makes it an attractive design in terms of its midspan performance.

The predicted and measured mixed-out loss coefficients at design and off-design Mach numbers are summarized in Table 6.6.

Table 6.6 Mixed-out loss coefficients at tested Mach numbers

\begin{tabular}{|c|c|c|c|c|c|c|c|c|c|c|c|}
\hline & \multicolumn{3}{|c|}{ SL1 } & \multicolumn{3}{|c|}{ SL2 } & \multicolumn{3}{|c|}{ SL2P } & SL3 & SL4 \\
\hline$M_{2, i s}$ & 0.65 & 0.78 & 0.91 & 0.65 & 0.78 & 0.91 & 0.65 & 0.78 & 0.90 & 0.78 & 0.78 \\
\hline$R e_{C, 2}$ & 480000 & 580000 & 680000 & 500000 & 600000 & 690000 & 490000 & 590000 & 670000 & 580000 & 620000 \\
\hline \multicolumn{12}{|l|}{ CFD } \\
\hline $\boldsymbol{Y}$ & 0.037 & 0.037 & 0.035 & 0.033 & 0.032 & 0.030 & 0.033 & 0.032 & 0.031 & 0.031 & 0.029 \\
\hline$\zeta$ & 0.033 & 0.031 & 0.028 & 0.029 & 0.027 & 0.025 & 0.029 & 0.027 & 0.025 & 0.027 & 0.024 \\
\hline \multicolumn{12}{|l|}{ EXP } \\
\hline $\boldsymbol{Y}$ & 0.030 & 0.030 & 0.031 & 0.029 & 0.030 & 0.033 & 0.034 & 0.033 & 0.035 & 0.024 & 0.027 \\
\hline$\zeta$ & 0.026 & 0.025 & 0.024 & 0.026 & 0.025 & 0.025 & 0.030 & 0.028 & 0.027 & 0.020 & 0.023 \\
\hline
\end{tabular}

\subsubsection{Additional Effects of Mach Number and Reynolds Number on Profile Flows: CFD Results}

The effects of off-design operation on airfoil loading distributions and surface skin friction coefficients were presented in Section 6.4.2; the ensuing effects on the predicted loss coefficients are described here. The numerical predictions, however, should be interpreted with caution due to the aforementioned differences between the experiments and the CFD.

The effects of varying the exit Mach number between 0.6 and 1.0 on the total pressure deficit $\left(\Delta P_{0} / P_{01}\right)$ and energy loss coefficient $(\zeta)$ are presented in Figure 6.16. The Reynolds number was held constant at 600,000 for the Mach number study. The shifting of the wakes with Mach number is associated with the change in flow turning (Figure 6.11): the outlet flow angle increases almost linearly up to Mach 0.95 and then drops slightly at Mach 1.0. Similar flow angle variations were observed by Corriveau (2005) for other high-speed turbine cascades. 
In Figure 6.16, the total pressure deficit $\left(\Delta P_{0} / P_{01}\right)$ rises sharply with Mach number. At subsonic outlet Mach numbers, this rise is mainly due to additional entropy generation within the blade surface boundary layers, and larger mixing losses: absolute losses $\left(\Delta P_{0}\right)$ are proportional to the cube of the boundary layer edge velocities, which rise more rapidly than $P_{01}$. The energy loss coefficients, on the other hand, do not change significantly as the outlet Mach number is increased in the subsonic range. Therefore, expressing the entropy generation as a fraction of the outlet kinetic energy, as described in Chapter 4, produces loss coefficients that are nearly Mach number independent in the subsonic range according to the computations.
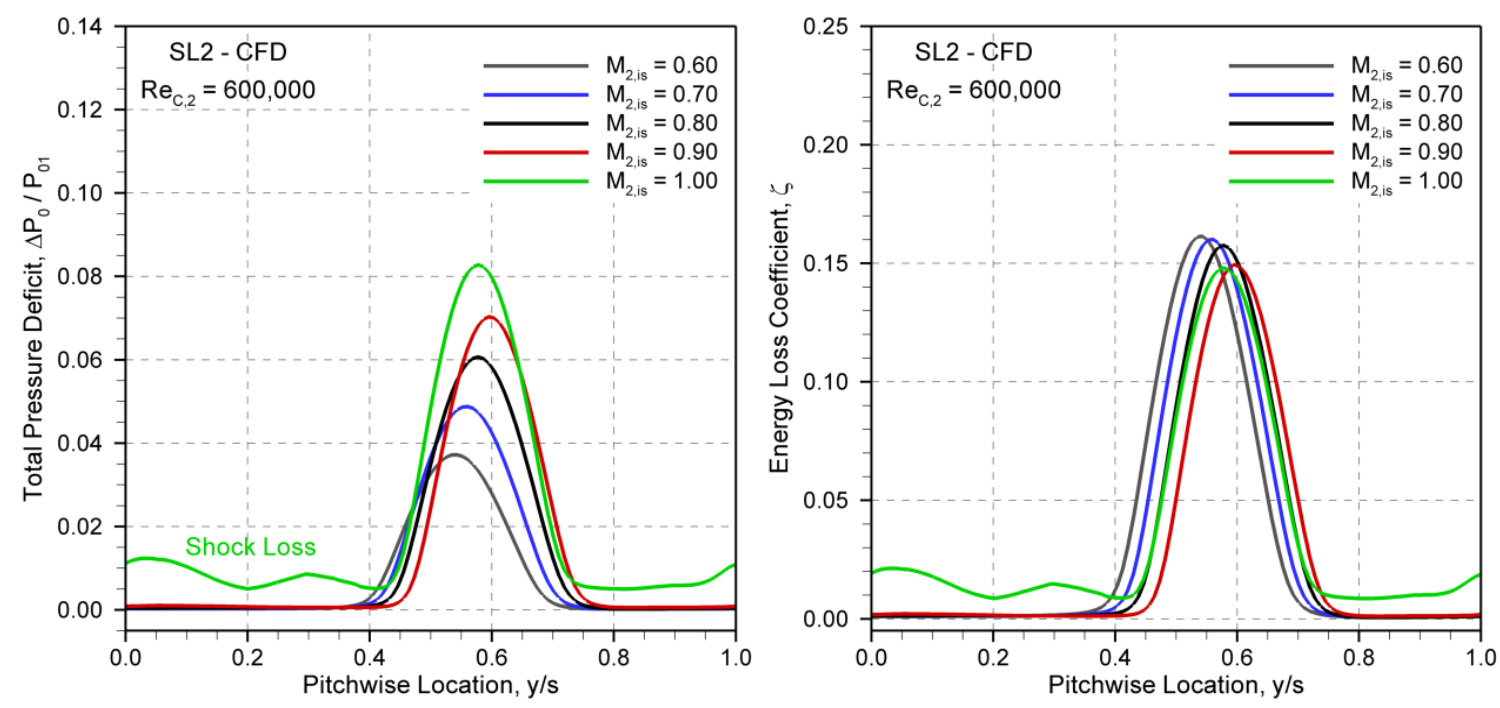

Figure 6.16 Effects of Mach number variation for SL2 at $2.0 C_{X}: \Delta P_{d} / P_{01}$ (left) and $\zeta$ (right)

The variations of the predicted mixed-out loss coefficients with Mach number are presented in Figure 6.17. As the exit Mach number is raised from 0.5 to 0.9 , the total pressure deficit, $\Delta P_{0} / P_{01}$, increases almost linearly, whereas the energy loss coefficient slightly decreases. The total pressure loss coefficient $(Y)$ remains nearly constant between Mach 0.5 and Mach 0.9 for this particular airfoil. At higher Mach numbers, all loss coefficients increase due to the formation of shock waves and this is especially evident at Mach 1.0, as anticipated from Figure 6.16.

The effects of Reynolds number variation on the predicted mixed-out profile loss coefficients at the design Mach number $\left(\mathrm{M}_{2, \text { is }}=0.8\right)$ are presented in Figure 6.18. The loss coefficients decrease by about $10 \%$ as the outlet Reynolds number is raised from 
400,000 to 800,000 . This loss reduction is consistent with the blade loading predictions in Figure 6.12: the blade surface boundary layers remain attached and the skin coefficients decrease with Reynolds number. Based on these results in the subsonic regime, the Reynolds number seems to have a similar influence on the predicted energy loss coefficients as the Mach number. Therefore, the reductions in numerical losses with Mach number in Figure 6.15 are also attributed to the increase in Reynolds number.

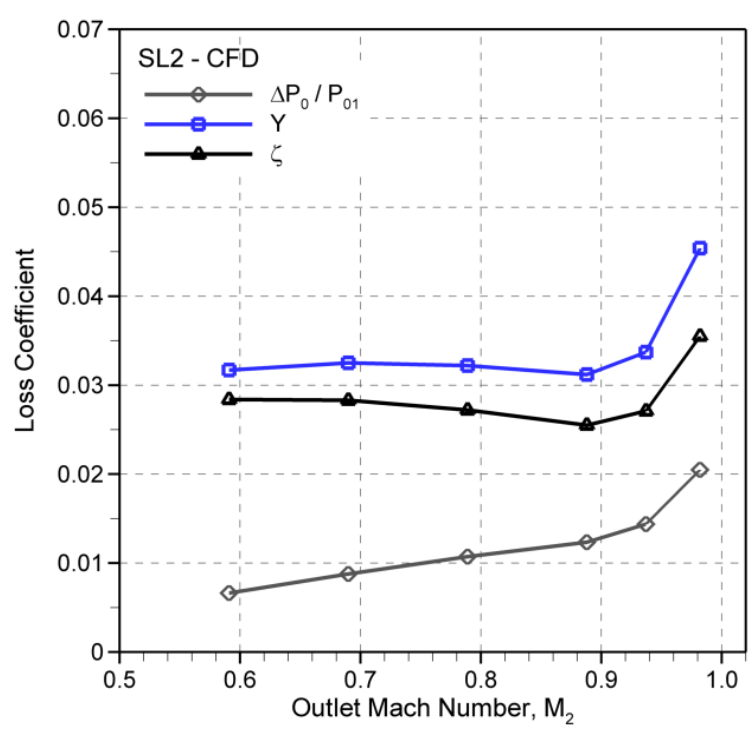

Figure 6.17 The effects of Mach number variation on the predicted loss coefficients for SL2 $\left(\operatorname{Re}_{C, 2}=600,000\right)$

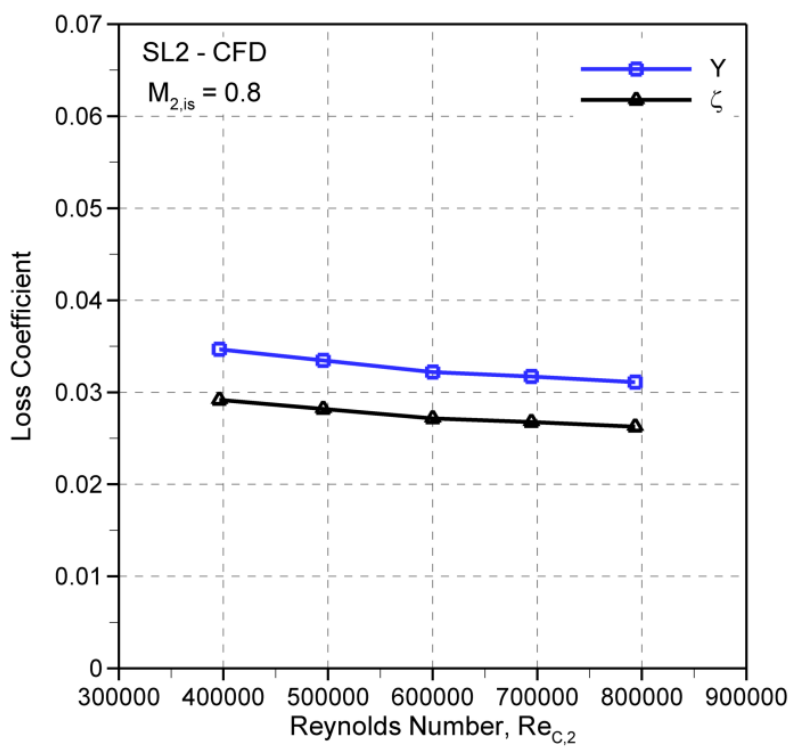

Figure 6.18 The effects of Reynolds number variation on the predicted loss coefficients for $\operatorname{SL2}\left(M_{2, i s}=0.8\right)$ 


\subsubsection{Comparisons with Kacker and Okapuu (1982) Loss Correlation}

The experimental results at the design Mach number are compared with the loss predictions by the Kacker and Okapuu (K-O) loss correlation in this section. The K-O loss correlation is a modification of the correlation proposed by Ainley and Mathieson (1951) to account for the improvements in turbine design from the 1950s through the 1970s. The K-O loss correlation is selected here for the comparisons since it is still widely used by gas turbine engine manufactures, such as PWC. As will be seen, however, the K-O correlation overpredicts the losses of the SL family of airfoils due to their relatively large pitch-to-chord ratios and large outlet flow angles (considered more "modern" designs).

The Ainley and Mathieson (A-M) profile loss correlation is defined as:

$$
Y_{A-M}=Y_{P, A-M} \cdot Y_{T E T}
$$

where $Y_{P, A-M}$ is the predicted loss coefficient for a turbine airfoil with trailing edge thickness-to-throat ratio $(T E T / o)$ of 0.02 . If TET/o is different than $0.02, Y_{T E T}$ is then used to correct for trailing edge thickness effects. The value of $Y_{P, A-M}$ is estimated using Equation 6.3 based on the loss correlations for axial-entry nozzles $\left(\beta_{1}=0\right)$ and impulse blades $\left(\beta_{1}=\beta_{2}\right)$.

$$
Y_{P, A-M}=\left\{Y_{P\left(\beta_{1}=0\right)}+\left(\frac{\beta_{1}}{\beta_{2}}\right)^{2}\left[Y_{P\left(\beta_{1}=\beta_{2}\right)}-Y_{P\left(\beta_{1}=0\right)}\right]\right\}\left(\frac{t_{\max } / C}{0.2}\right)^{\frac{\beta_{1}}{\beta_{2}}}
$$

The values of the profile loss coefficients, $Y_{P\left(\beta_{1}=\beta_{2}\right)}$ and $Y_{P\left(\beta_{1}=0\right)}$, in Equation 6.3 are determined form the curves in Figure 6.19. As shown, each curve corresponds to a different exit flow angle $\left(\beta_{2}\right)$. The solid lines are based on actual experimental data mainly from low-speed cascades, whereas the dashed lines represent the extrapolated trends for larger outlet flow angles. It is immediately apparent that nozzles, shown on the left, generally produce lower losses than impulse blades, shown on the right, due to smaller total flow turning. It is also evident that increasing the outlet flow angle results in larger losses, particularly for impulse blades since it is accompanied by an identical increase in inlet flow angle. Finally, the location of the lowest loss coefficient on each curve indicates the optimum pitch-to-chord ratio. 
The symbols in Figure 6.19 represent the loss predictions for the SL family of airfoils, based on their geometric and aerodynamic parameters. Due to the relatively large outlet flow angles $\left(63^{\circ}\right.$ and $\left.69^{\circ}\right)$, and large pitch-to-chord ratios (0.8 to 1.0), the estimated losses appear in the extrapolated zones for impulse blades. The resulting $Y_{P}$ predictions, which are significantly higher than the measured values, are presented in Table 6.7. Notwithstanding the large numerical differences, the A-M profile loss correlation generally captures the correct trend in the data.
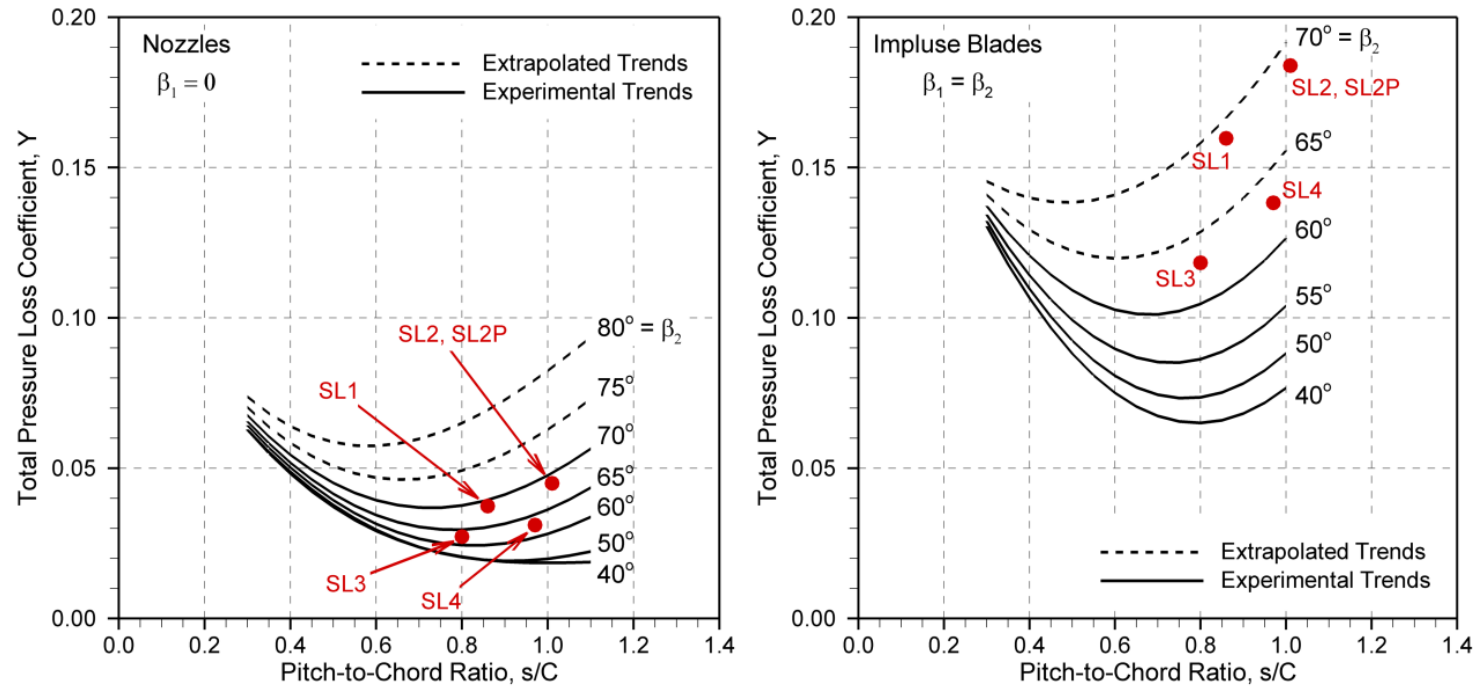

Figure 6.19 Ainley and Mathieson (1951) profile loss correlations for nozzles (left) and impulse blades (right)

Table 6.7 Comparisons with the A-M profile loss correlation $\left(M_{2}=0.8\right)$

\begin{tabular}{|c|c|c|c|c|c|c|c|c|}
\hline & $\boldsymbol{\beta}_{\boldsymbol{1}}$ & $\boldsymbol{\beta}_{\boldsymbol{2}}$ & $\boldsymbol{s} / \boldsymbol{C}$ & $\boldsymbol{t}_{\boldsymbol{m a x}} / \boldsymbol{C}$ & $\boldsymbol{Y}_{\boldsymbol{P}, \text { Nozzle }}$ & $\boldsymbol{Y}_{\boldsymbol{P}, \text { Impulse }}$ & $\boldsymbol{Y}_{\boldsymbol{A}-\boldsymbol{M}}$ & $\boldsymbol{Y}_{\text {Measured }}$ \\
\hline SL1 & 43.5 & 69.1 & 0.86 & 0.25 & 0.037 & 0.160 & 0.099 & 0.030 \\
\hline SL2 & 43.5 & 68.2 & 1.01 & 0.22 & 0.045 & 0.184 & 0.109 & 0.030 \\
\hline SL2P & 43.5 & 68.5 & 1.01 & $0.38^{*}$ & 0.045 & 0.184 & 0.116 & 0.033 \\
\hline SL3 & 27.0 & 62.9 & 0.80 & 0.24 & 0.027 & 0.118 & 0.048 & 0.024 \\
\hline SL4 & 27.0 & 63.0 & 0.97 & 0.23 & 0.031 & 0.138 & 0.054 & 0.027 \\
\hline
\end{tabular}

The maximum airfoil thickness-to-chord ratio (last term in Equation 6.3) is limited to 0.25 for the SL2P loss prediction (actual $t_{\max } / C=0.38$ identified with an asterisk in Table 6.7), as recommended by Ainley and Mathieson (1951).

The K-O system proposes a few modifications to the A-M loss correlation to take the following form: 


$$
\begin{gathered}
Y_{P, K-O}=\left\{Y_{P\left(\beta_{1}=0\right)}+\left|\frac{\beta_{1}}{\beta_{2}}\right|\left(\frac{\beta_{1}}{\beta_{2}}\right)\left[Y_{P\left(\beta_{1}=\beta_{2}\right)}-Y_{P\left(\beta_{1}=0\right)}\right]\right\}\left(\frac{t_{\text {max }} / C}{0.2}\right)^{\frac{\beta_{1}}{\beta_{2}}} \\
Y_{K-O}=\left[0.914\left(\frac{2}{3} K_{P} Y_{P, K-O}+Y_{\text {Shock }}\right)\right] f(R e)+Y_{T E T}^{\prime}
\end{gathered}
$$

- $\left|\beta_{1} / \beta_{2}\right|$ allows for negative inlet angles

- $\quad 0.914$ factor removes the trailing edge thickness contribution (replaced with $Y_{T E T}^{\prime}$ )

- $\quad 2 / 3$ factor accounts for lower loss production due to improvements in design

- $K_{P}$ is the subsonic Mach number correction

- $\quad Y_{\text {Shock }}$ is the supersonic drag rise for $M_{2}>1.0$

- $f(R e)$ is the Reynolds number correction for $\operatorname{Re}_{C, 2}<200,000$ or $R e_{C, 2}>1,000,000$

- $\quad Y_{T E T}^{\prime}$ accounts for the additional losses due to the trailing edge thickness

The shock wave and Reynolds number effects in the correlation do not contribute to the current data sets. The trailing edge thickness contribution is found using Figure 6.20 and Equation 6.6 (presented in terms of $Y^{\prime}{ }_{T E T}$ for an outlet Mach number of 0.8).

$$
Y_{T E T}^{\prime}=Y_{T E T\left(\beta_{1}=0\right)}^{\prime}+\left|\frac{\beta_{1}}{\beta_{2}}\right|\left(\frac{\beta_{1}}{\beta_{2}}\right)\left[Y_{T E T\left(\beta_{1}=\beta_{2}\right)}^{\prime}-Y_{T E T\left(\beta_{1}=0\right)}^{\prime}\right]
$$

The subsonic Mach number correction, also shown in Figure 6.20, is applied as follows:

$$
K_{P}=1-1.25\left(\frac{M_{1}}{M_{2}}\right)^{2}\left(M_{2}-0.2\right)
$$

The K-O loss predictions and the measured profile losses are compared in Table 6.8, which also shows the contributions from the different components. The low-turning cascades display marginally better agreement with the correlations. The losses, however, are overestimated in all cases due to the combination of large outlet flow angles and large pitch-to-chord ratios: as indicated previously, the loss predictions are based on the extrapolated trends rather than actual correlated data. The higher ranges of these parameters are more commonly utilized today as the airfoil duty is increased to reduce the weight of the turbine stages (Popovic, 2005; Praisner et al., 2008). The current transonic data sets may be used in the future to improve the loss correlations for turbine airfoils with larger outlet flow angles. 

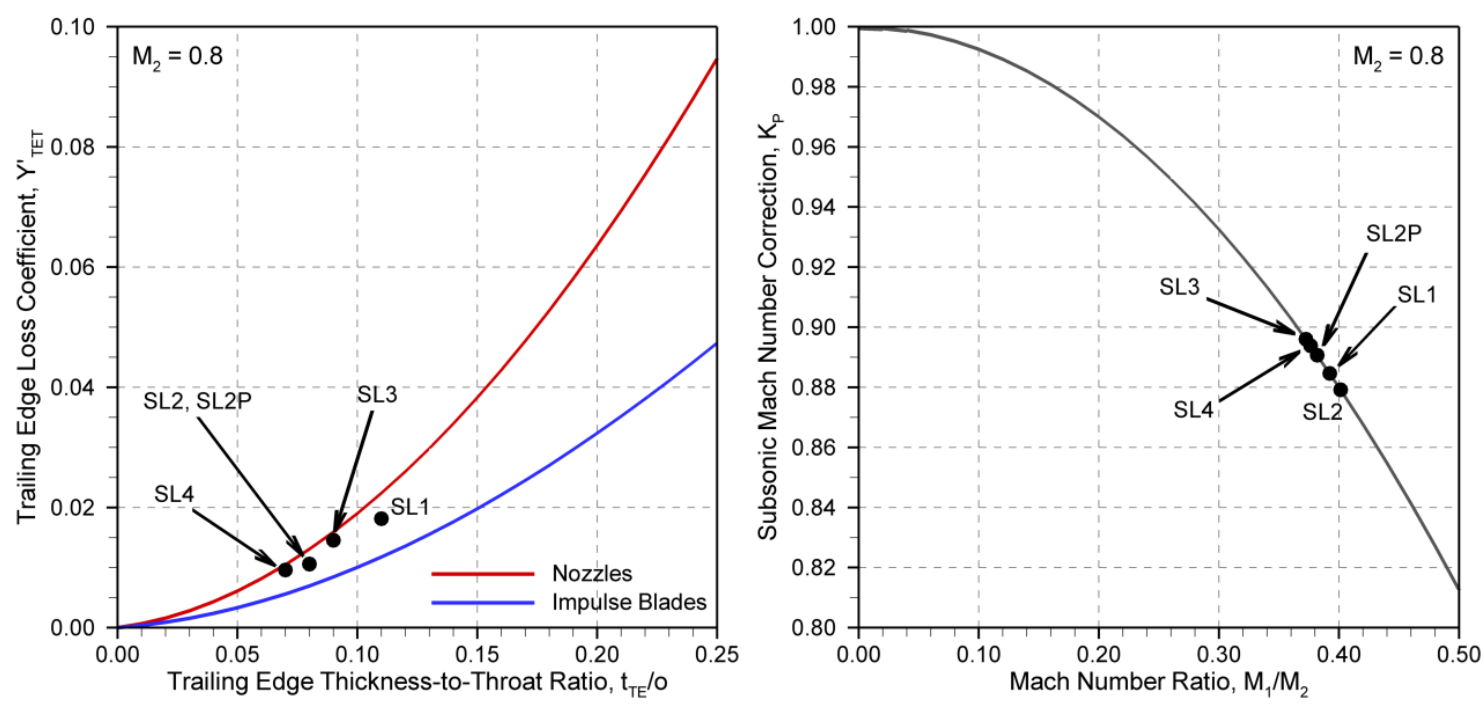

Figure 6.20 Components of the K-O loss correlation at $M_{2}=0.8$ : trailing-edge-thickness effects (left) and subsonic Mach number correction (right)

Table 6.8 Comparisons with the K-O profile loss correlation $\left(M_{2}=0.8\right)$

\begin{tabular}{|c|c|c|c|c|c|c|}
\hline & $\boldsymbol{Y}_{\boldsymbol{P}, \boldsymbol{K}-\boldsymbol{O}}$ & $\boldsymbol{K}_{\boldsymbol{P}}$ & $\mathbf{0 . 9 1 4 \cdot 2 / 3} \cdot \boldsymbol{K}_{\boldsymbol{P}} \cdot \boldsymbol{Y}_{\boldsymbol{P}, \boldsymbol{K} \text { - }}$ & ${\boldsymbol{\boldsymbol { Y } ^ { \prime }}}_{\boldsymbol{T E T} \boldsymbol{T}}$ & $\boldsymbol{Y}_{\boldsymbol{K}-\boldsymbol{O}}$ & $\boldsymbol{Y}_{\text {Measured }}$ \\
\hline SL1 & 0.099 & 0.89 & 0.054 & 0.018 & 0.072 & 0.030 \\
\hline SL2 & 0.109 & 0.90 & 0.059 & 0.011 & 0.070 & 0.030 \\
\hline SL2P & 0.116 & 0.89 & 0.063 & 0.011 & 0.074 & 0.033 \\
\hline SL3 & 0.048 & 0.88 & 0.025 & 0.015 & 0.040 & 0.024 \\
\hline SL4 & 0.054 & 0.88 & 0.029 & 0.010 & 0.039 & 0.027 \\
\hline
\end{tabular}




\section{Chapter 7}

\section{Secondary Flows and Losses in Turbine Cascades with Flat Endwalls}

\subsection{Introduction}

Modern turbine design is strongly influenced by the cost and the weight of the engine, demanding fewer turbine blades and higher blade loadings. Increasing the blade loadings, however, may result in higher total pressure losses, which are in part manifested as higher secondary losses. Secondary flow research has received considerable attention in the past and excellent reviews have been provided by Dunham (1970), Sieverding (1985) and Langston (2001). Several recent numerical studies have shown the ability of 3D Reynolds-Averaged Navier-Stokes (RANS) codes to predict reasonably reliably the formation of the main vortical structures within the blade passages, and thereby to provide trend-wise accurate results for the losses (Pecnik et al., 2005; Praisner et al., 2007; Hjärne et al., 2007). On the other hand, accurate prediction of the total pressure losses and the associated mixing processes still remains challenging. The introduction of flow compressibility effects adds an additional level of difficulty. The current research project is aimed to improve the understanding of the loss generating mechanisms in compressible secondary flows, including the effects of endwall contouring.

The midspan flow aerodynamics of the turbine cascades were examined in Chapter 6; the endwall flows and the secondary losses for the cases with flat endwalls are discussed here. The results are presented in two sections: low flow turning (SL3F and SL4F) at the design Mach number, and high flow turning (SL1F and SL2F) at both design and offdesign Mach numbers. As described in Chapter 6, varying the exit Mach number in the experiments is accompanied with changes in Reynolds number and row velocity ratio; these parameters also affect the off-design secondary flow characteristics. 
The discussions in this chapter are primarily based on the pressure probe measurements and the surface flow visualization results. The experimental results from the high-turning cascades are also compared with fully-turbulent CFD predictions at the design Mach number. The CFD simulations were performed following the completion of the experiments to closely replicate the inlet and outlet boundary conditions, including the inlet endwall boundary layers.

The inlet endwall boundary layers are documented here since the state of the boundary layer affects the formation of the secondary flows. The airfoil loading measurements are also briefly examined here to assess the effects of loading distribution on secondary losses.

\subsection{Inlet Endwall Boundary Layers}

As shown by de la Rosa Blanco et al. (2003), the state of the inlet endwall boundary layer (laminar, transitional or turbulent) affects the formation of the secondary flows and the production of the secondary losses. The inlet total pressure deficit in the endwall boundary layer is also required to determine the blade row loss generation (net loss production within the blade passage). In the current study, the endwall boundary layers were traversed using a Pitot probe $(D=0.9 \mathrm{~mm})$ upstream of the cascades $\left(-2.0 C_{X}\right)$ at all investigated Mach numbers. The total pressure distribution from the Pitot probe was combined with the static pressure from the wall static tap, assuming negligible spanwise pressure gradients; the results are presented in Figure 7.1 in terms of the inlet Mach number ratio $\left(M_{1} / M_{C L}\right)$. The boundary layer profiles are similar in all cases, including the off-design Mach number studies, since the inlet Mach numbers do not vary significantly.

The integrated endwall boundary layer parameters are presented in Table 7.1, and indicative of fully turbulent, zero-pressure-gradient boundary layers for all cases $(H \approx 1.4$ and $\left.R e_{\theta}>10,000\right)$. The small differences in the integrated values are mainly attributed to the upstream contraction in the wind tunnel, which is a function of inlet flow angle, blade pitch and the number of blade passages (Chapter 3). Raising the Mach number results in slightly thinner inlet endwall boundary layers. Net secondary losses have been found to be insensitive to inlet endwall boundary layer thickness in previous experiments by Chen et al. (1986) and Gregory-Smith et al. (1988a). 

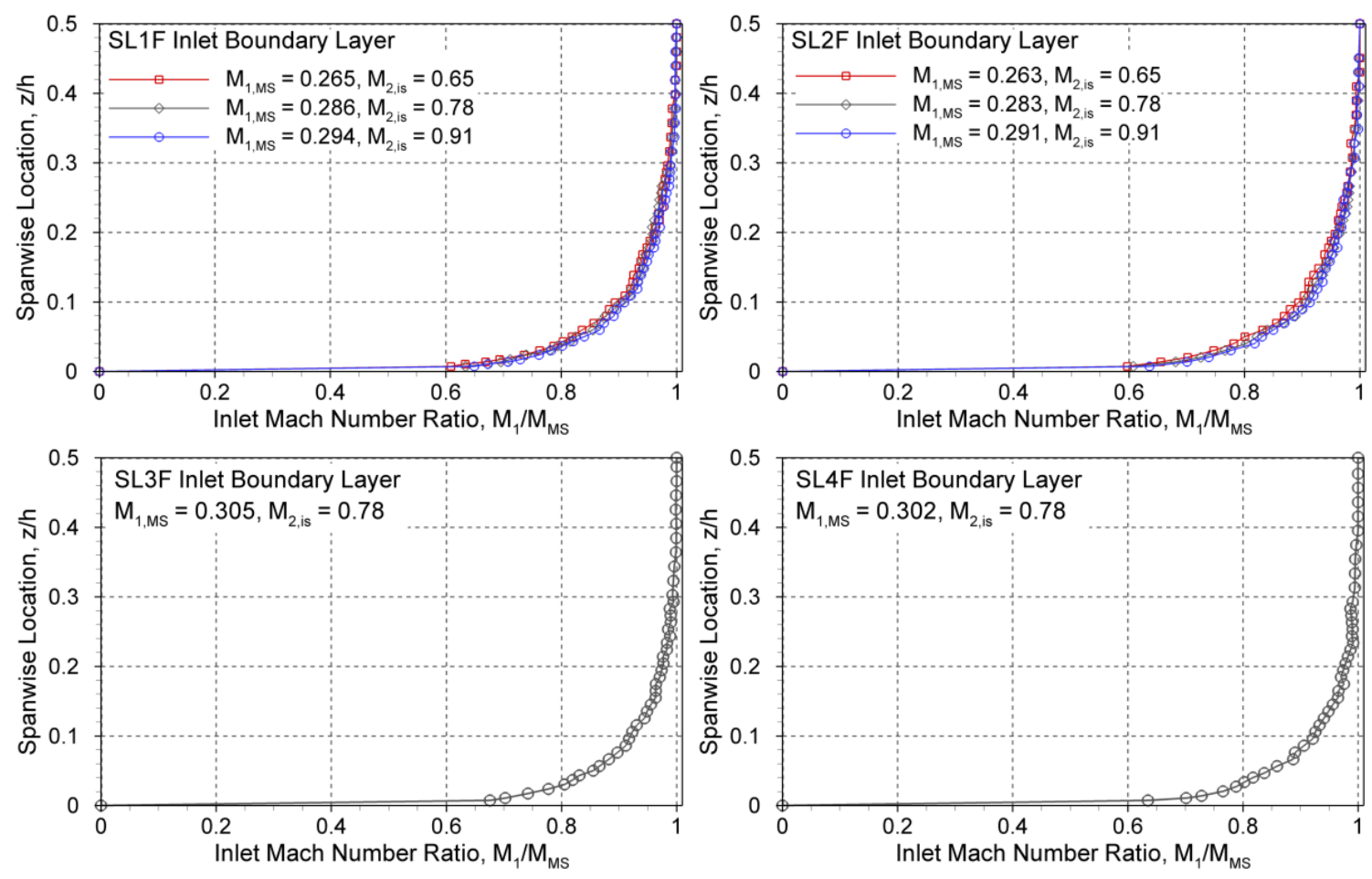

Figure 7.1 Inlet endwall boundary layer measurements

Table 7.1 Integrated endwall boundary layer parameters for tested Mach numbers

\begin{tabular}{|c|c|c|c|c|c|c|c|c|c|c|c|}
\hline & $M_{1, M S}$ & $M_{2, i s}$ & $\begin{array}{l}\delta_{99 \%} \\
(\mathrm{~mm})\end{array}$ & $\delta_{99 \%} / h$ & $\begin{array}{c}\delta^{*} \\
(\mathrm{~mm})\end{array}$ & $\delta^{\star} / h$ & $\begin{array}{c}\theta \\
(m m)\end{array}$ & $\theta / h$ & $H$ & $\boldsymbol{R e _ { \delta }}$ & $\operatorname{Re}_{\theta}$ \\
\hline \multirow{3}{*}{ SL1F } & 0.265 & 0.65 & 18 & 0.30 & 2.05 & 0.034 & 1.47 & 0.024 & 1.4 & 15,500 & 11,100 \\
\hline & 0.286 & 0.78 & 17 & 0.28 & 1.94 & 0.032 & 1.40 & 0.023 & 1.4 & 17,000 & 12,200 \\
\hline & 0.294 & 0.91 & 16 & 0.26 & 1.80 & 0.029 & 1.29 & 0.021 & 1.4 & 17,700 & 12,700 \\
\hline \multirow{3}{*}{ SL2F } & 0.263 & 0.65 & 18 & 0.30 & 2.15 & 0.035 & 1.54 & 0.025 & 1.4 & 16,600 & 11,862 \\
\hline & 0.283 & 0.78 & 17 & 0.28 & 1.95 & 0.032 & 1.39 & 0.023 & 1.4 & 17,500 & 12,400 \\
\hline & 0.291 & 0.90 & 16 & 0.26 & 1.88 & 0.031 & 1.35 & 0.022 & 1.4 & 18,500 & 13,300 \\
\hline SL3F & 0.305 & 0.78 & 15 & 0.25 & 1.60 & 0.026 & 1.13 & 0.019 & 1.4 & 15,200 & 10,700 \\
\hline SL4F & 0.302 & 0.78 & 14 & 0.23 & 1.61 & 0.026 & 1.13 & 0.019 & 1.4 & 14,900 & 10,400 \\
\hline
\end{tabular}

\subsection{Results from the Low-Turning Cascades: SL3F and SL4F}

\subsubsection{Turbine Cascades}

The low-turning turbine cascades are referred to as SL3F and SL4F. The airfoils have the same inlet and outlet design flow angles with $90^{\circ}$ total flow turning, but different 
aerodynamic loading levels: SL4F has a Zweifel coefficient of 1.01, which is 30\% higher than that for SL3F. Table 7.2 summarizes the geometric and aerodynamic parameters of the turbine cascades at design conditions (mixed-out flow parameters at midspan).

Table 7.2 Summary of cascade parameters: low-turning airfoils

\begin{tabular}{|c|c|c|c|c|c|c|c|c|c|c|c|c|}
\hline & \multicolumn{2}{|c|}{$C(\mathrm{~mm})$} & \multicolumn{2}{|c|}{$C_{x}(\mathrm{~mm})$} & $h(\mathrm{~mm})$ & \multicolumn{2}{|c|}{$o(\mathrm{~mm})$} & $s(\mathrm{~mm})$ & \multicolumn{2}{|c|}{$t_{\max }(\mathrm{mm})$} & \multicolumn{2}{|c|}{$S\left(^{\circ}\right)$} \\
\hline SL3 & & 0.8 & 25. & & 61.0 & 10 & & 24.6 & 7.4 & & 34 & \\
\hline \multirow[t]{2}{*}{ SL4 } & \multicolumn{2}{|c|}{32.4} & \multicolumn{2}{|c|}{25.4} & 61.0 & \multicolumn{2}{|c|}{13.7} & 31.4 & \multicolumn{2}{|c|}{7.4} & \multicolumn{2}{|c|}{38.3} \\
\hline & $M_{1}$ & $M_{2, \text { is }}$ & $M_{2}$ & $\beta_{1}\left({ }^{\circ}\right)$ & $\beta_{2}\left({ }^{\circ}\right)$ & $Z w$ & $A V D R$ & $A V R$ & $R V R$ & $\operatorname{Re}$ & & $\operatorname{Re}_{c, 2}$ \\
\hline SL3F & 0.305 & 0.78 & 0.76 & 27.0 & 62.9 & 0.78 & 0.98 & 1.22 & 2.38 & 290 & & 580,000 \\
\hline SL4F & 0.302 & 0.78 & 0.77 & 27.0 & 63.0 & 1.01 & 0.98 & 1.24 & 2.43 & 300 & & 620,000 \\
\hline
\end{tabular}

\subsubsection{Midspan Blade Loading Distributions}

The midspan surface Mach number distributions are presented in Figure 7.2. These are repeated here for convenience and because of the importance of the loading distributions to secondary flows.
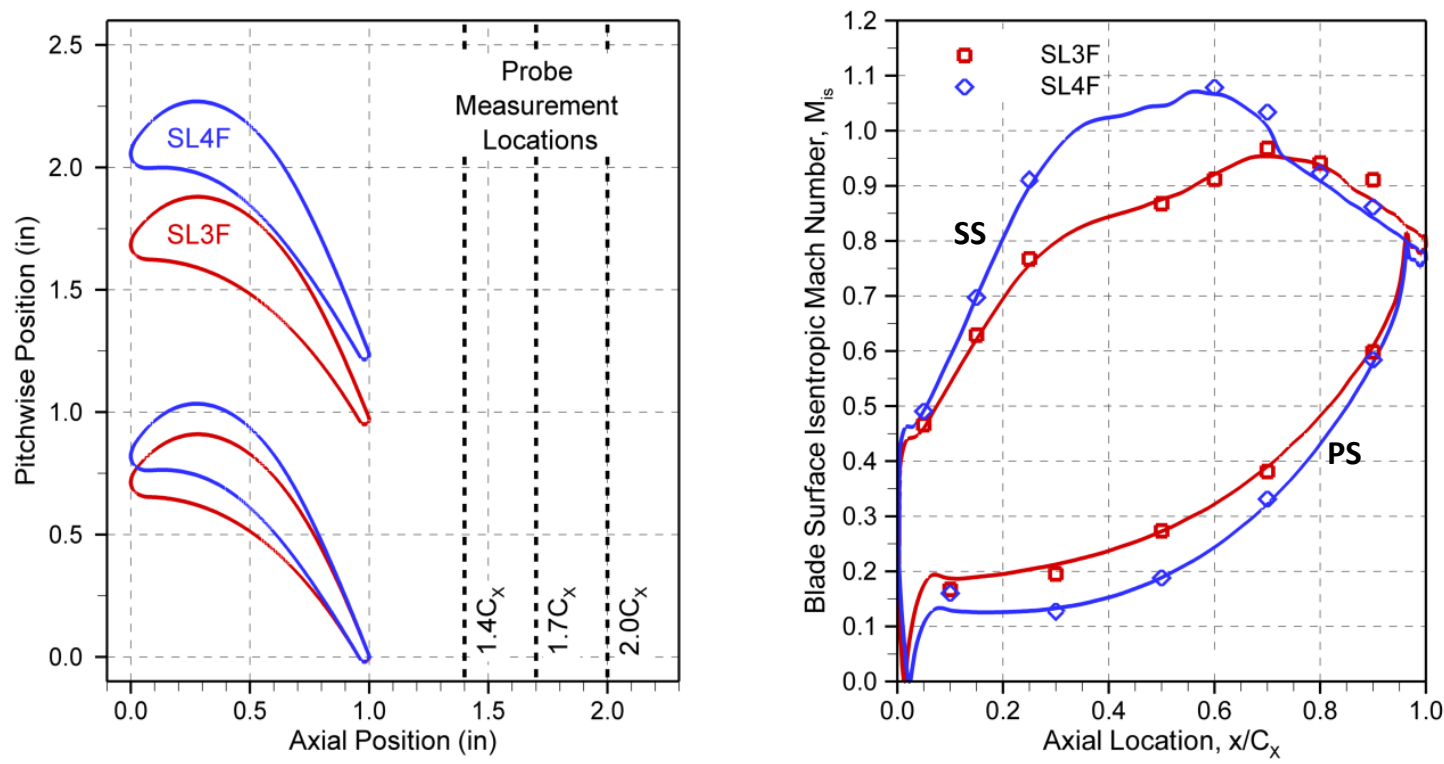

Figure 7.2 Low-turning airfoils: midspan blade loading distributions $\left(M_{2, i s}=0.78\right)$

SL4F is clearly more highly loaded than SL3F; since the two airfoils have the same inlet and outlet flow angles and Mach number ratio, the 30\% increase in Zweifel 
coefficient has been achieved by increasing the blade spacing. The larger blade spacing results in lower suction-surface static pressures on SL4F (i.e. local supersonic flow from $0.35 C_{X}$ to $0.7 C_{X}$ ), exposing the boundary layer to higher local velocities. Consequently, SL4F produces larger profile losses than SL3F, as discussed in Chapter 6. Increasing the Zweifel coefficient also requires slight changes to the loading distribution in an attempt to redistribute the additional adverse pressure gradients on the suction surface; SL4F exhibits more mid-loading characteristics than SL3F, which is considered aft-loaded.

\subsubsection{Downstream Flow Field Measurements at $1.4 C_{X}$}

As shown in Figure 7.2 and Figure 7.3, the main measurement plane was located at $140 \%$ axial chord lengths downstream of the leading-edge plane $\left(1.4 C_{X}\right)$. Additional measurements were collected at $1.7 C_{X}$ and $2.0 C_{X}$. The measurement grid is also shown in Figure 7.3, where SS and PS indicate suction and pressure sides of the wake, respectively. At every spanwise position, 41 evenly-spaced pitchwise data points were collected. The closest traverse to the endwall was limited to 3\% span to avoid wall interference effects on probe measurements (details provided in Chapter 3).
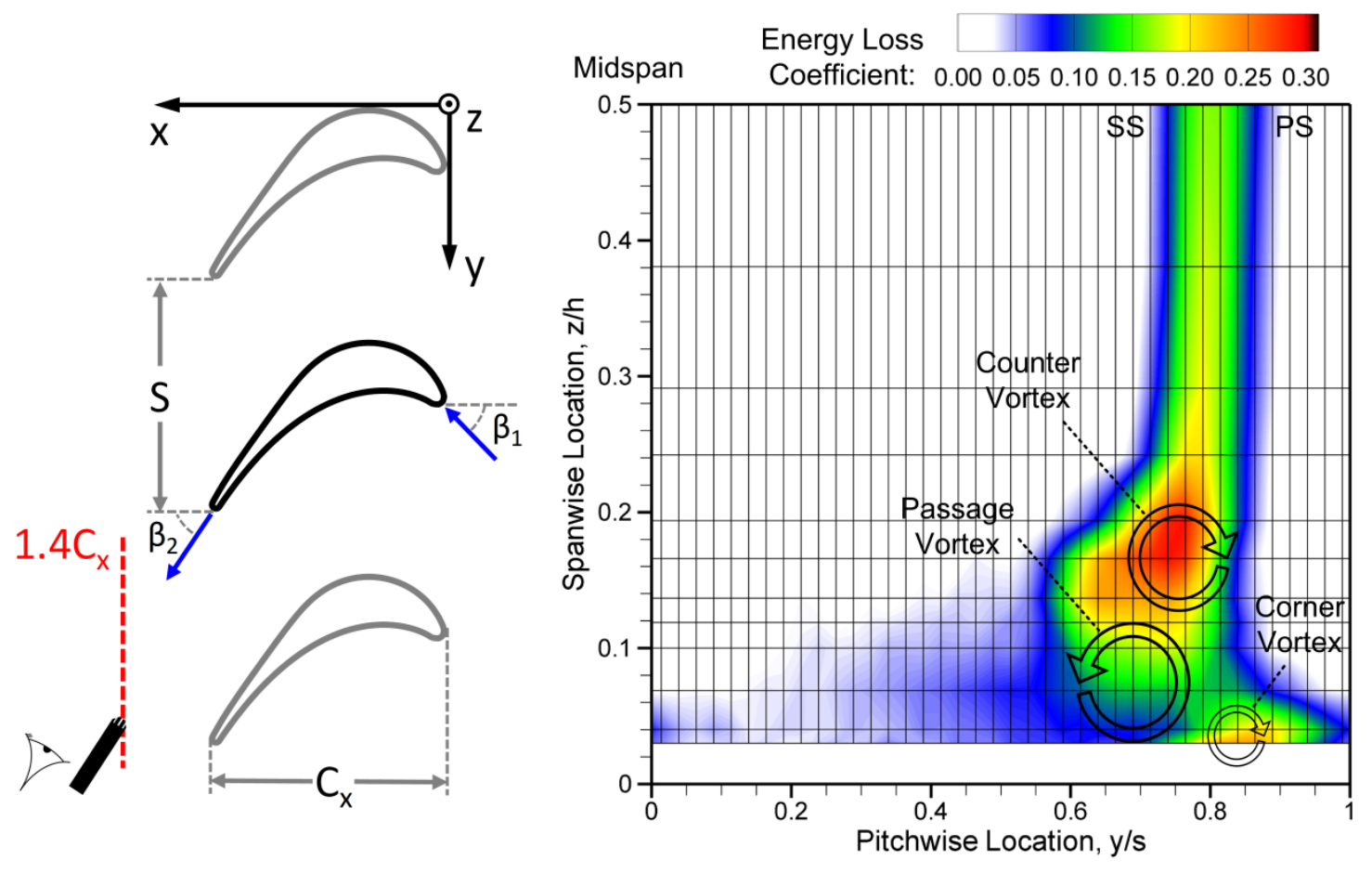

Figure 7.3 Downstream measurement plane and secondary flow features 
Figure 7.4 shows colour contour plots of energy loss coefficient for SL3F and SL4F at the design Mach number. The core, loss-free flow regions are displayed in white and the high-loss regions in red. The results are plotted over one blade pitch $(0<y / s<1)$ and half $\operatorname{span}(0<z / h<0.5)$ with appropriate aspect ratios (the aspect ratios are based on the values of pitch and span, presented in Table 7.2). SL4F displays higher profile losses, increased secondary flow penetration, and larger peak energy loss coefficients: $\zeta=0.29$ for SL4F versus 0.26 for SL3F. SL4F also exhibits higher losses near the endwall associated with a larger corner vortex. The corner vortex does not seem to be captured for SL3F.

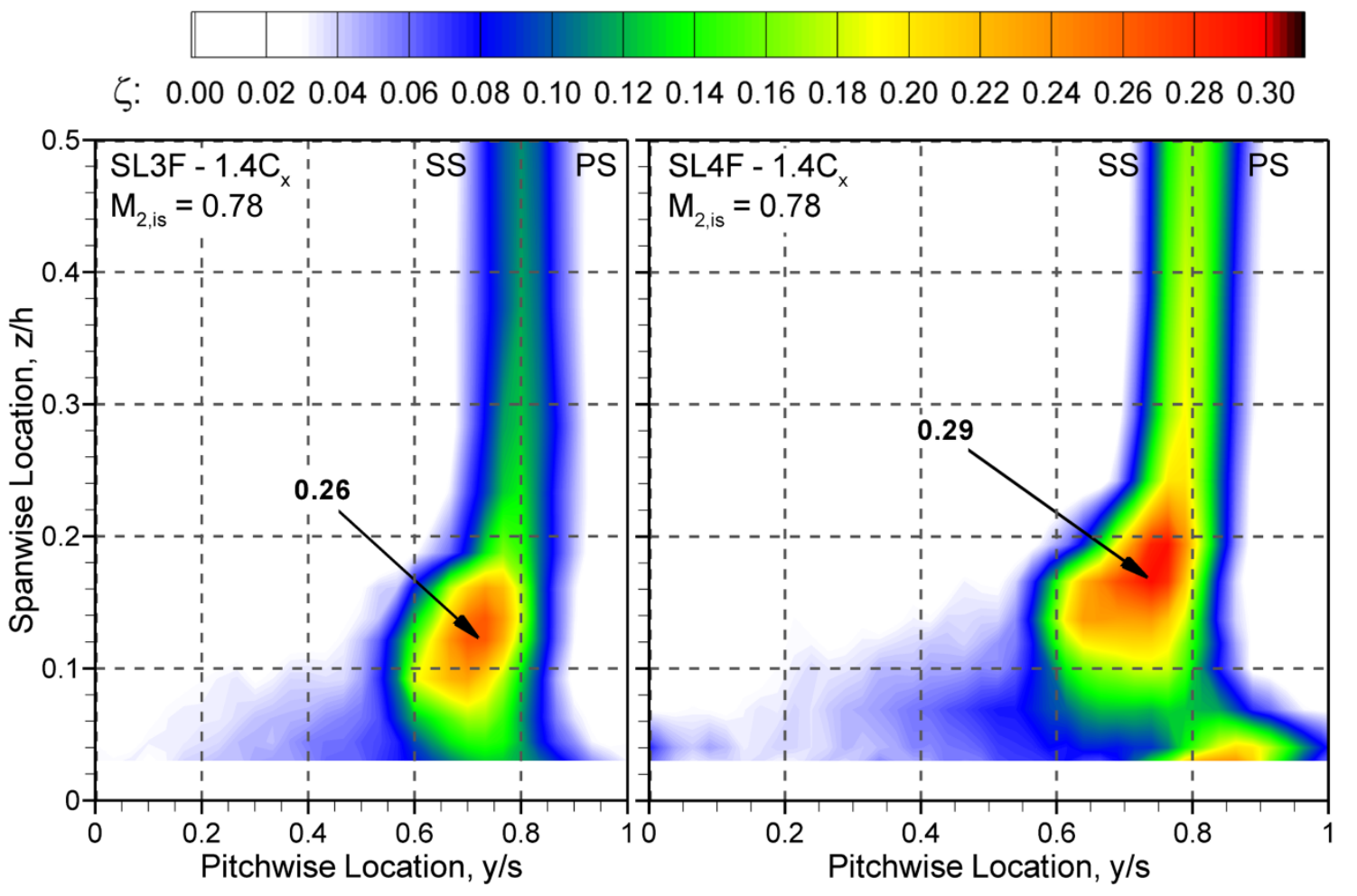

Figure 7.4 Low-turning cascades: energy loss coefficient contour plots

The energy loss coefficient is defined relative to the inlet free-stream total pressure. This is appropriate for the profile flow region. However, the total pressure deficit within the secondary flow region includes fluid originating from the inlet endwall boundary layer. To obtain the net secondary loss, both profile losses and inlet boundary layer losses are subtracted from the mass-averaged total pressure deficit downstream. These results are presented in Section 7.5. 
The total pressure losses are mass-averaged to account for mass flux non-uniformities in the measurement plane (see Chapter 4 for details). In view of this, colour floods of local axial velocity density ratio, representative of local mass flux, superimposed over contour lines of energy loss coefficient are presented in Figure 3.1(a).

(a)

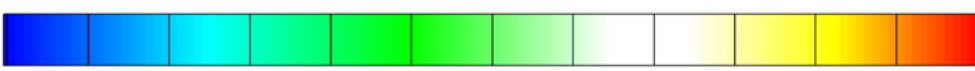

AVDR $_{\text {local }}: 0.60 \quad 0.650 .700 .750 .800 .850 .900 .951 .001 .051 .101 .15 \quad 1.20$

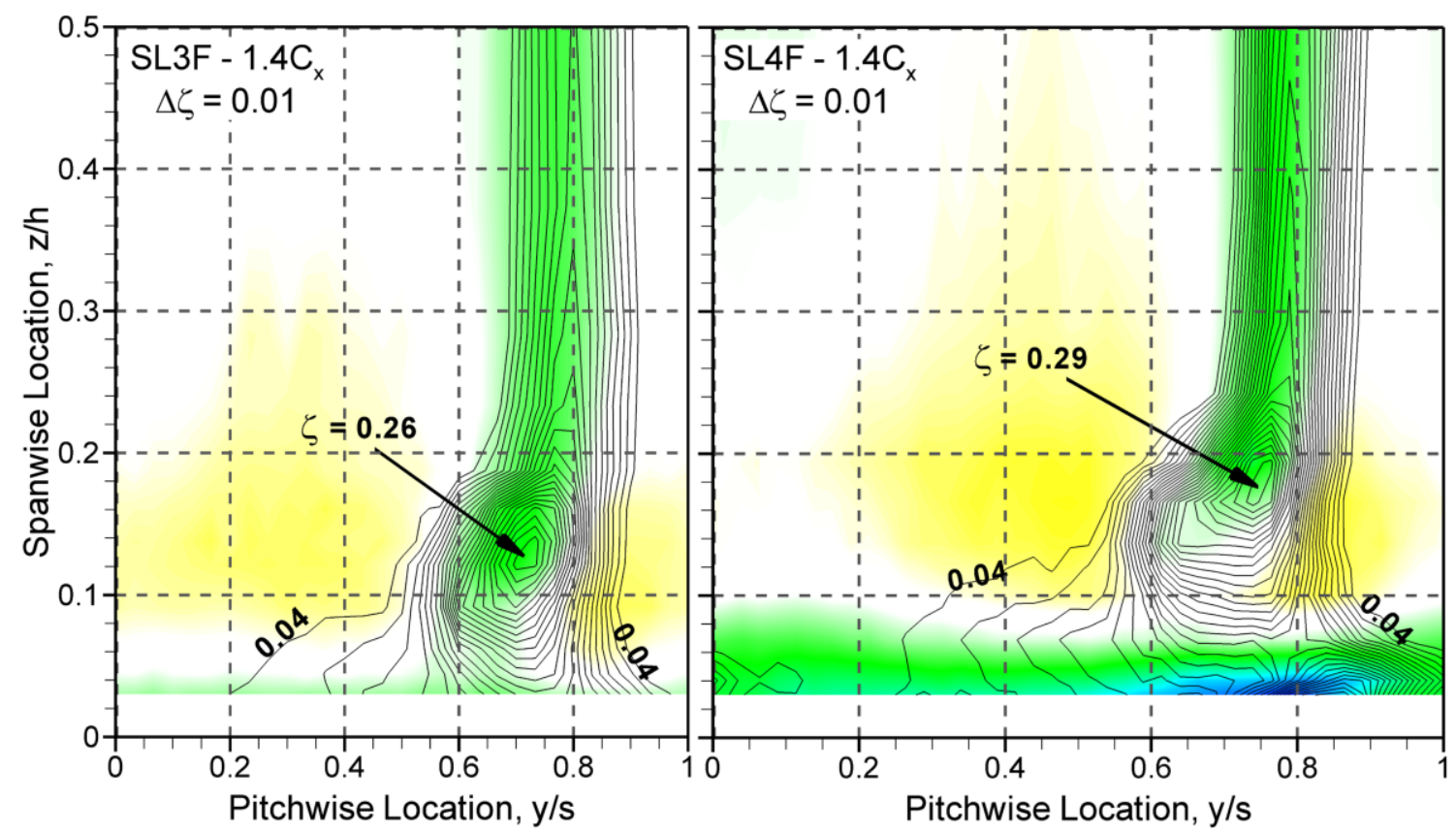

(b)

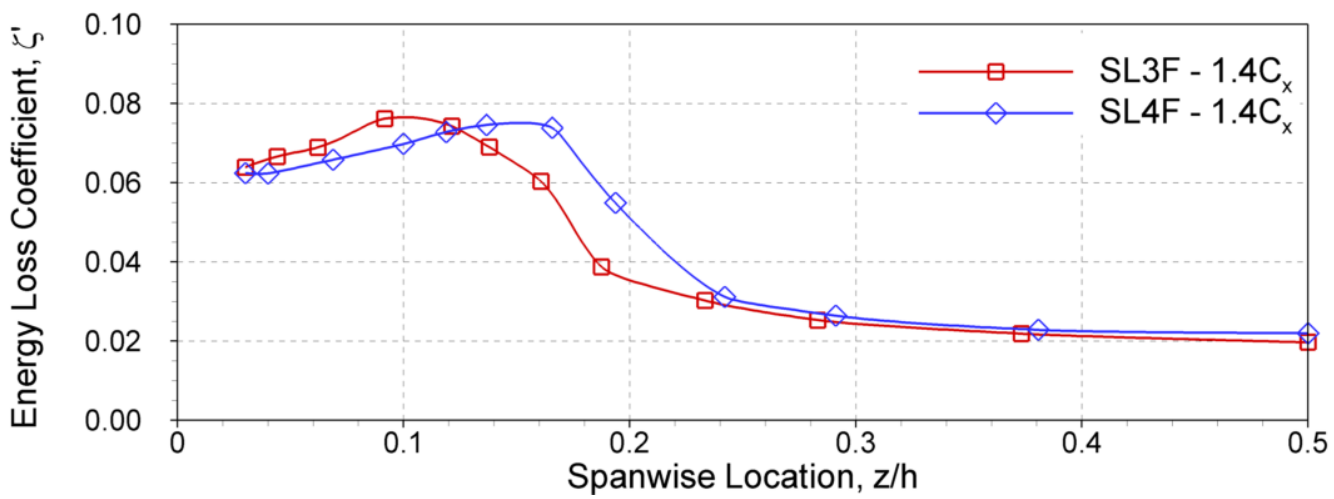

Figure 7.5 (a) Colour floods of local axial velocity density ratio superimposed over contour lines of energy loss coefficient, and (b) pitch-averaged energy loss coefficients

Mass flow deficit is apparent in the midspan wakes $\left(A V D R_{\text {local }}<1\right)$, and in the secondary flow region. Excess mass fluxes $\left(A V D R_{\text {local }}>1\right)$ are generated through two mechanisms: core flow acceleration around the "blocked" secondary loss region, and 
flow underturning induced by the passage vortex. Figure 3.1(a) shows that higher mass fluxes mainly occur within the core flow regions $(0<y / s<0.6$ and $z / h>0.1)$, which does not affect the mass-averaged losses. There is, however, a small area where total pressure losses coincide with high mass fluxes: $0.8<y / s<0.9$ and $0.1<z / h<0.2$. This is attributed to the underturning effects of the passage vortex. Near the endwall, SL4F shows significantly smaller mass fluxes as a result of large flow overturning by the passage vortex and the mass flow deficit in the near-wall fluid. Although this region coincides with high endwall losses, the contribution to the overall mass-averaged results is reduced by the low mass fluxes.

The pitchwise mass-averaged energy loss coefficients $\left(\zeta^{\prime}\right)$ are shown in Figure 3.1(b) to show the aforementioned effects quantitatively. SL4F displays higher losses than SL3F from approximately $12 \%$ to $50 \%$ span. From $3 \%$ to $12 \%$ span, on the other hand, SL4F produces lower mass-averaged losses as a result of the smaller mass fluxes. Overall, SL4F produces higher mass-averaged profile losses $(+10 \%)$ and higher row losses $(+6 \%)$ than $\mathrm{SL} 3 \mathrm{~F}$ at $1.4 C_{X}$, as shown in Section 7.5.

Most low-speed studies have identified the passage vortex as the dominant secondary flow structure responsible for generating total pressure losses (Langston, 2001). Furthermore, the contributions of the counter and corner vortices to overall loss production depend partly on the size and position of the passage vortex. Therefore, an objective of the current study was to examine the influence of the passage vortex on loss production in compressible flows.

Colour-flood contour plots of streamwise vorticity coefficient $\left(C_{\omega s}\right)$ are presented in Figure 7.6(a) to identify the size and location of the vortical structures. The contour lines of energy loss coefficient are also overlaid for comparisons. Based on the right-handed rule, the passage vortex has positive vorticity, whereas the counter and corner vortices have negative vorticity. The presences of passage and counter vortices are evident in both contour plots. The corner vortex, on the other hand, does not appear for SL3F, likely due to its close proximity to the endwall (within 0 to $3 \%$ span). SL4F displays stronger vortical structures overall, and the passage vortex in particular is significantly more intense. 
(a)

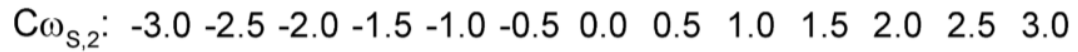

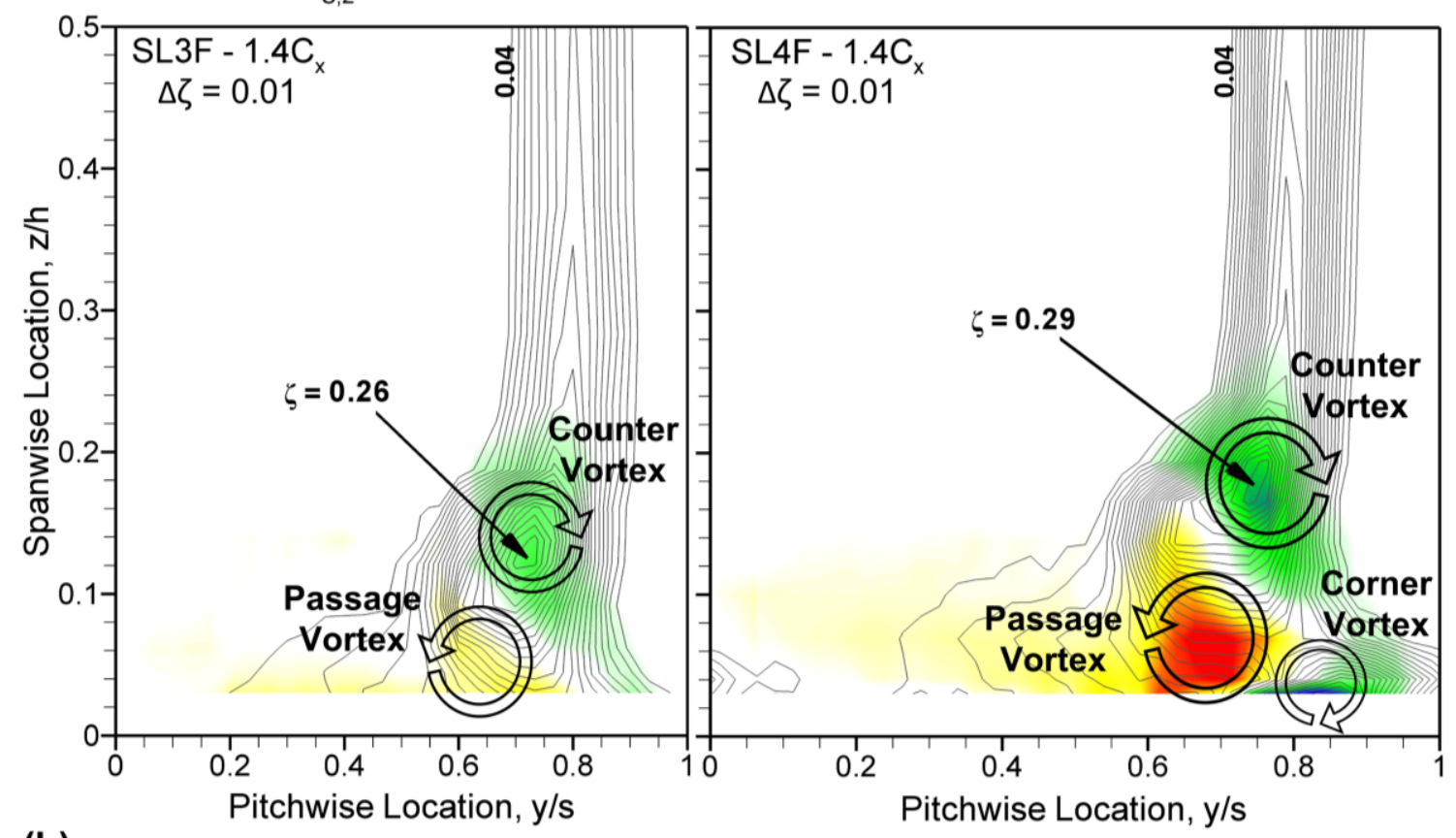

(b)

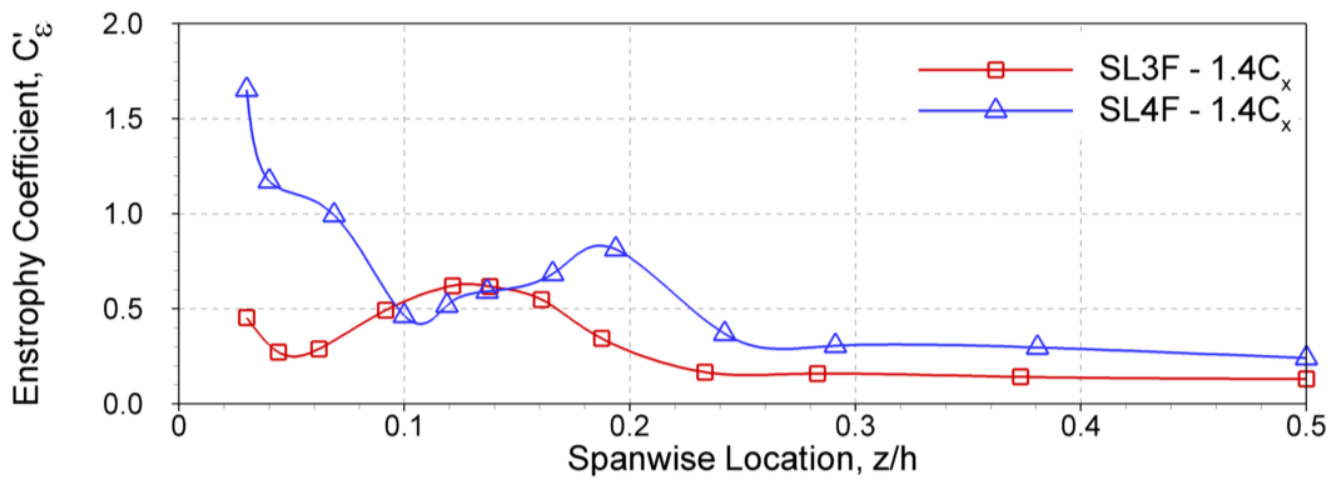

(c)

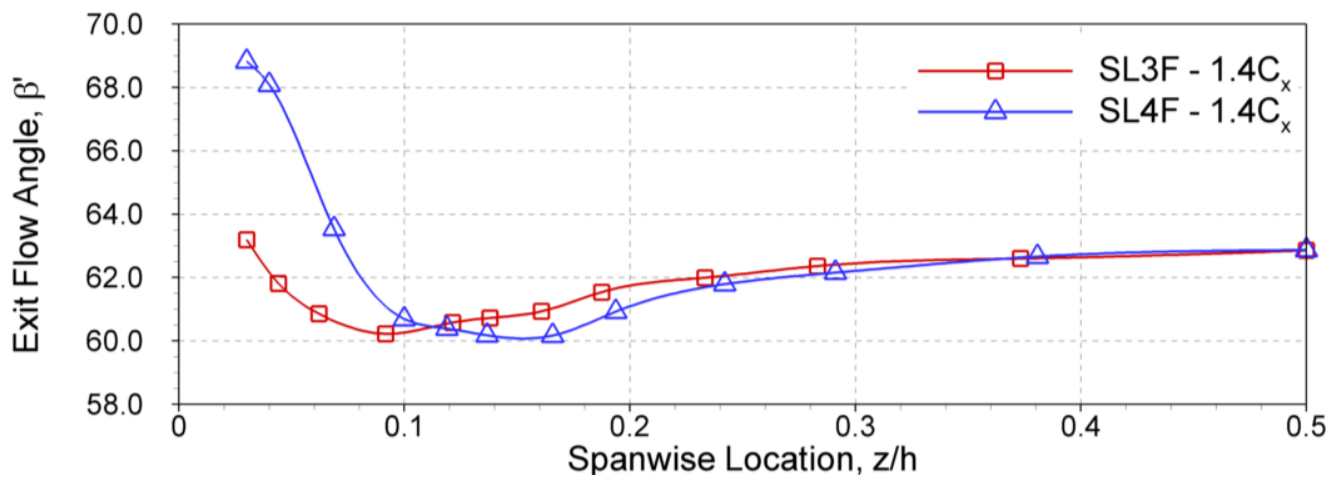

Figure 7.6 (a) Streamwise vorticity coefficients, (b) pitch-averaged enstrophy coefficients and (c) exit flow angles 
Even though the streamwise development of the vortices has not been documented in the present study, some tentative conclusions about their formation within the passage can still be drawn based on the loading distributions in Figure 7.2 and the vorticity contour plots. Increased loading in SL4F results in higher cross-passage pressure gradients, especially in the mid part of the passage. As shown by Yamamoto (1987a; 1987b) and Benner et al. (2004), the growth of the pressure-side leg of the horseshoe vortex, which later merges with the passage vortex, is strongly influenced by the transverse pressure gradients in the forward and mid parts of the passage. Here, SL4F exhibits a more intense passage vortex, potentially resulting in stronger interaction with the suction-surface boundary layer and thus higher total pressure losses within the blade row. Additional losses are generated as a result of the dissipation of the passage vortex downstream of the cascade (suggesting higher downstream losses for SL4F). The locations of the strongest loss cores in Figure 7.6(a) are seen to coincide with the positions of the counter vortices. The losses, however, are not entirely generated by the action of the counter vortices; the peak losses are partially attributed to the funnelling of the inlet boundary layer fluid up the suction surface as the flow approaches the trailing edge. In other words, the counter vortex may play a more important role in loss redistribution than loss production. Further losses close the endwalls are generated as a result of the entrainment of the low-momentum endwall boundary layer fluid by the passage vortex and the corner vortex, and the subsequent mixing with the free-stream flow. Consequently, the larger corner vortex for SL4F results in additional endwall losses compared to SL3F. As previously noted, however, the contributions of the nearwall losses to the mass-averaged results are reduced for SL4F by the smaller mass fluxes.

The pitch-averaged enstrophy coefficients are shown in Figure 7.6(b) to compare the vorticity results more quantitatively. As discussed in Chapter 4, enstrophy is related to the kinetic energy of the vortical structures in the flow field, and like mechanical energy, enstrophy is destroyed by viscous forces (Davidson, 2005). The low enstrophy coefficients $\left(C_{\varepsilon}\right)$ between midspan and $25 \%$ span are associated with low vorticity in the two-dimensional wake region. There are two distinct peaks of $C_{\varepsilon}$ in SL4F: one at approximately $19 \%$ span due to the counter vortex, and a second at 3\% span attributable to the passage vortex and the corner vortex. Overall, SL4F generates significantly higher 
enstrophy (by a factor of two) than SL3F, which will likely result in higher losses downstream of the measurement plane due to stronger shear flows.

As shown in Figure 7.6(c), streamwise vorticity also affects the exit flow angle variations. The two cascades show similar levels of underturning, albeit at different spanwise locations: SL4F shows higher secondary flow penetration than SL3F. On the other hand, SL4F displays higher flow overturning $\left(+6^{\circ}\right)$ near the endwall, induced by the stronger passage vortex. Very close to the endwall $(z / h=0.03)$, flow overturning is slightly reduced by the action of the counter-rotating corner vortex in SL4F. SL3F is also expected to generate flow overturning, but perhaps closer to the endwall (within 0 to $3 \%$ span) to balance the mass flux surplus associated with flow underturning, thereby satisfying continuity. In an engine environment, the larger exit flow angle variations downstream of SL4F would adversely affect the performance of the subsequent blade rows due to off-design incidence effects (i.e. higher losses would be expected from identical downstream blade rows).

Another important parameter related to the production of secondary losses is the secondary kinetic energy ( $S K E)$, associated with the vortical structures. Figure 7.7(a) shows colour floods of secondary kinetic energy coefficient $\left(C_{S K E}\right)$, overlaid on secondary velocity vectors $\left(\overrightarrow{U_{s e c}}=\overrightarrow{v_{s e c}}+\overrightarrow{w_{s e c}}\right)$. The secondary velocity vectors are normalized by the exit isentropic velocity $\left(U_{2, i s}\right)$.

CFD simulations often adequately predict the formation and growth of the vortical structures, but lack in modelling of the mixing processes leading to total pressure losses. Consequently, $C_{S K E}$ is a useful parameter to indicate the total pressure losses still to be generated downstream of the plane in question. $C_{S K E}$ is predominantly a function of the secondary velocities, and to a lesser extent of the local variations in density (Chapter 4). The magnitudes of the secondary velocities are directly proportional to the strength of the vortical structures as measured by their size and peak vorticity; largest secondary velocities appear in the vortex-vortex interaction regions in Figure 7.7(a). Secondary velocities in the core flow region $(20 \%$ to $60 \%$ pitch) result from the redirection of the free-stream fluid around the "blocked" secondary loss area. In Figure 7.7(a), SL4F displays significantly higher levels of $C_{S K E}$ than SL3F, which indicates that $S K E$ mixing out downstream of $1.4 C_{X}$ will generate higher losses in SL4F than in SL3F. The 
pitchwise mass-averaged $C_{S K E}$ plot in Figure 7.7(b) shows large differences between the two cascades from $3 \%$ to $6 \%$ span, which are attributed to the stronger passage vortex and the corner vortex for SL4F.

(a)
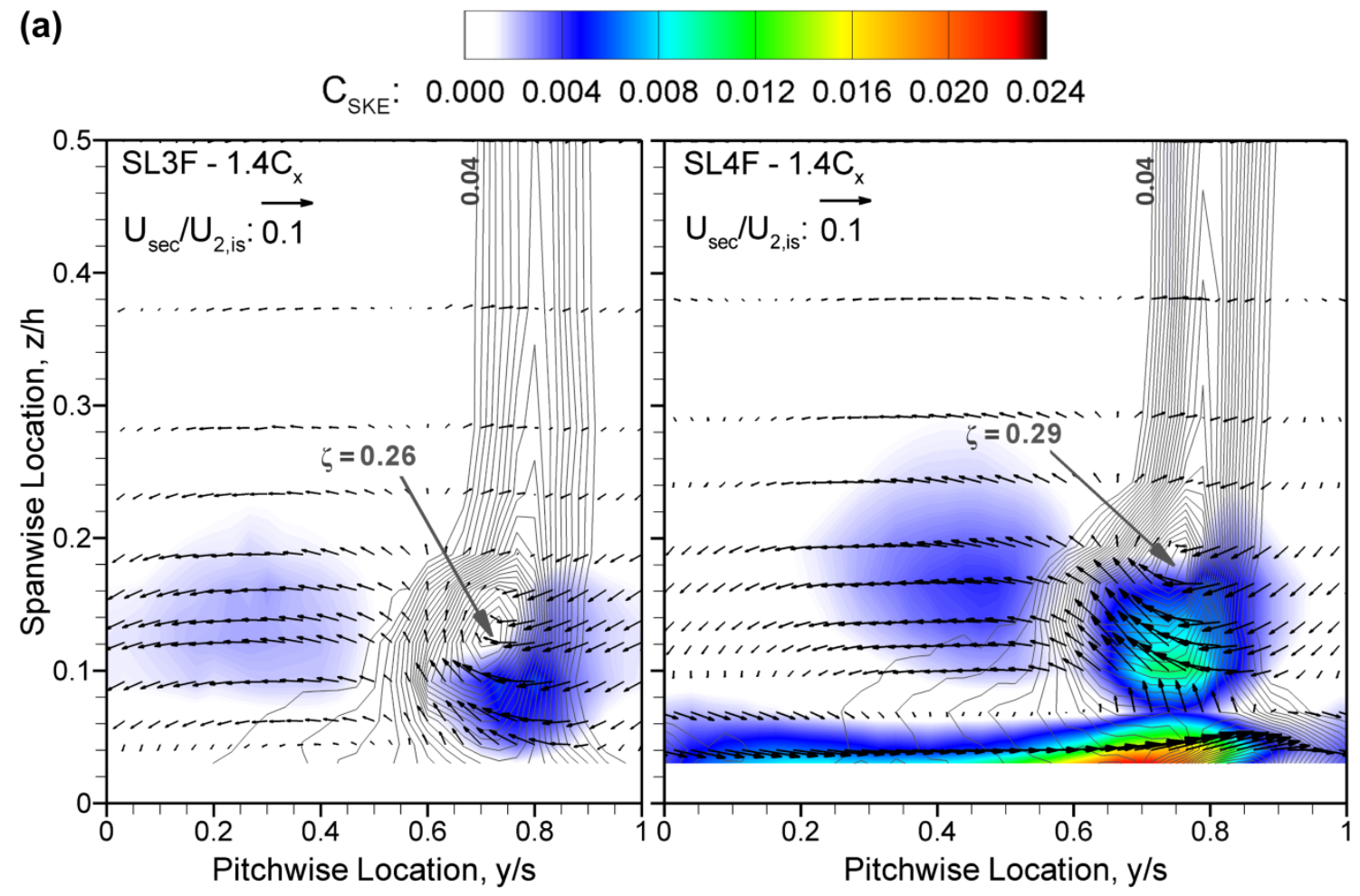

(b)

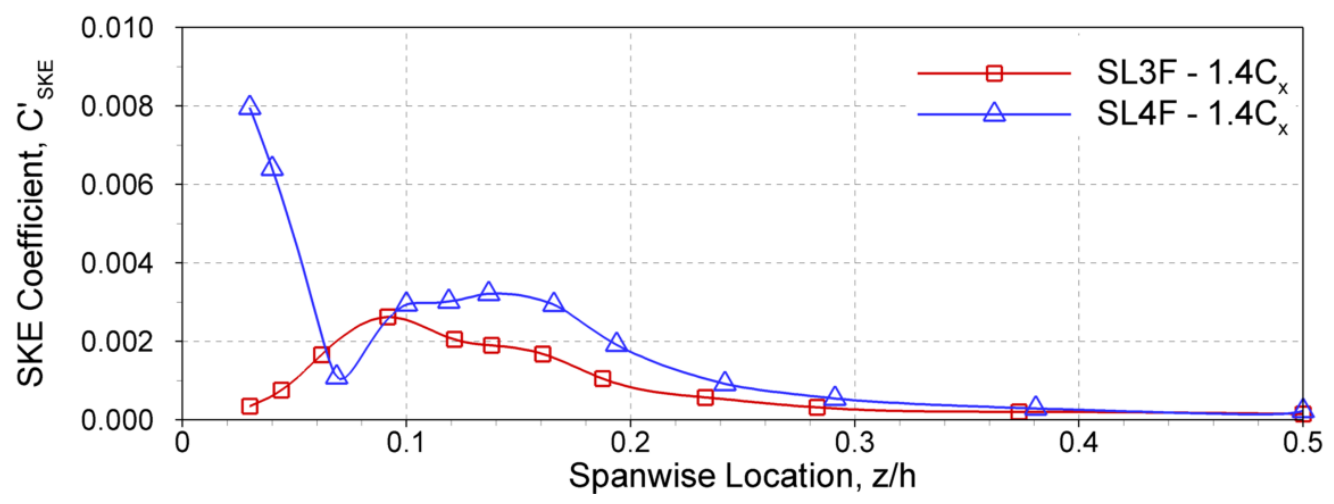

Figure 7.7 (a) Secondary kinetic energy coefficient contour plots, and (b) pitch-averaged results at $1.4 C_{X}$

\subsubsection{Downstream Growth of Secondary Losses}

Additional probe measurements were collected downstream of SL3F to document the development of the secondary flows. Results from three measurement planes are presented in Figure 7.8: $1.4 C_{X}, 1.7 C_{X}$ and $2.0 C_{X}$. Here, the local variations in mass flux 
have been incorporated into the loss coefficient contour plots (i.e. $\zeta \times A V D R_{\text {local }}$ ). Also shown are the secondary velocity vectors, depicting the movement and diffusion of the vortical structures downstream of the cascade. The corresponding pitch-averaged results are presented in Figure 7.9 for more quantitative comparisons.

The contour plots in Figure 7.8 show increasingly more uniform flow fields at farther downstream locations. The mixing out of the profile flow causes significant diffusion of the midspan wake, but only slight increases in profile losses at $1.7 C_{X}$ and $2.0 C_{X}$. This is consistent with the findings of Jouini (2000) and Corriveau (2005), as the mixing of the blade suction-surface and pressure-surface boundary layers predominantly takes place immediately after the trailing edge plane (between $1.0 C_{X}$ and $1.4 C_{X}$ ). The secondary losses, on the other hand, increase downstream of the cascade via several mechanisms: endwall boundary layer growth, entrainment of the endwall boundary layer fluid by the vortical structures and dissipation of the vortices away from the endwalls.

As previously discussed, the measurements for SL3F at $1.4 C_{X}$ only display part of the passage vortex, and do not capture a corner vortex (due to close proximity of the secondary flow structures to the endwall). Consequently, the SL3F results generally display very low levels of secondary kinetic energy at $1.4 C_{X}$. Additional downstream measurements show similar levels of mass-averaged $C_{S K E}$, but considerably higher row losses at $1.7 C_{X}$ and $2.0 C_{X}(+7 \%$ and $+16 \%$, respectively). In this case, the additional downstream losses are largely attributed to the entrainment of the low-momentum endwall boundary layer fluid by the passage vortex, based on measurements between 3\% and $50 \%$ span.

Secondary loss generation under incompressible flows typically correlates well with the production and dissipation of secondary kinetic energy (Knezevici, 2011). In the present compressible flows, it appears that the dissipation of the primary kinetic energy may play a more important role in secondary loss production, particularly in the near-wall region; similar observations were reported by Perdichizzi (1990) and Perdichizzi et al. (1990). This is of course not a general conclusion as it primarily depends on the positions of the vortices relative to the endwall downstream of the cascade. However, this observation suggests that displacing the passage vortex away from the endwall through implementation of endwall contouring to control secondary flows may result in 

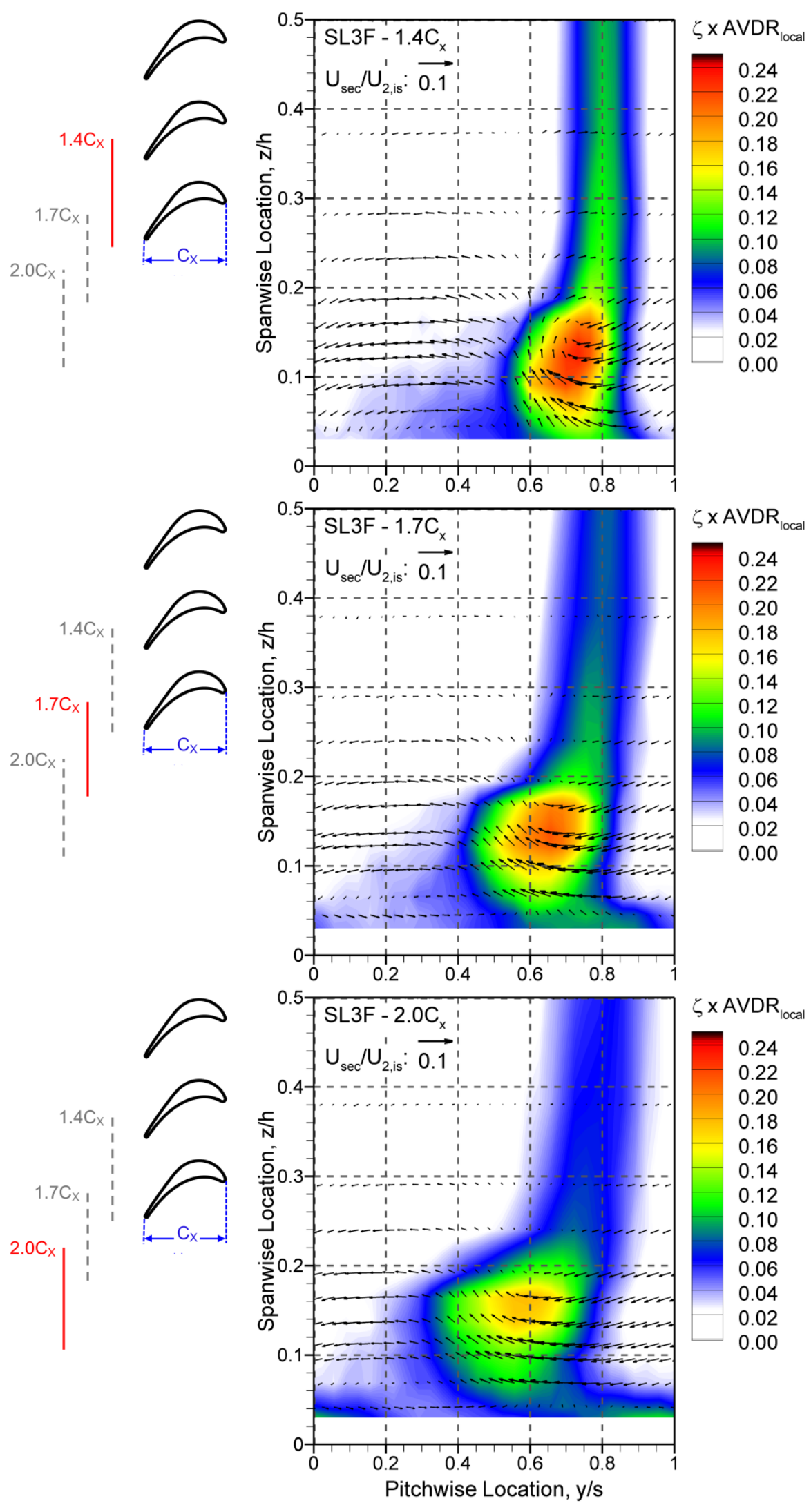

Figure 7.8 Downstream development of secondary loss distributions for SL3F 
smaller downstream losses. The conventional procedure for calculating mixed-out losses (Amecke and Safarik, 1995) does not account for the additional endwall loss generation since it assumes that there are no shear stresses at the walls of the control volume. As shown in previous high-speed cascade studies (Sieverding and Wilputte, 1981; Bassi et al., 1987; Perdichizzi, 1990; Dominy and Harding, 1990), the passage vortex typically migrates towards the endwall as the downstream Mach number is raised, thereby amplifying the endwall loss production. Further discussions of the effects of flow compressibility on secondary losses, based on additional Mach numbers, are provided in the next sections.

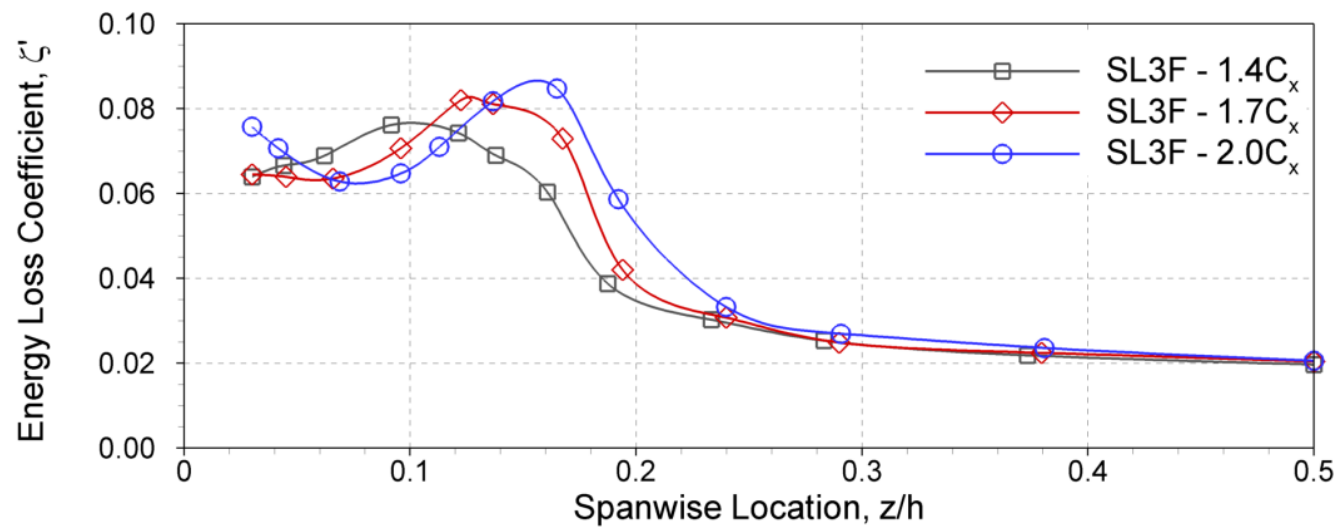

Figure 7.9 Pitch-averaged energy loss coefficients for SL3F

The integrated loss coefficients for SL3F and SL4F are presented in Table 7.3. As previously indicated, SL4F produces higher row losses and higher secondary kinetic energy than SL3F. The mass-averaged and mixed-out results for all cascades at both design and off-design conditions are summarized in Section 7.5.

Table 7.3 Mass-Averaged Results for SL3F and SL4F at 1.4C $C_{X}$

\begin{tabular}{|c|c|c|}
\hline & SL3F & SL4F \\
\hline Mass-Averaged SKE Coefficient, $\boldsymbol{C}^{\text {" }}$ sKE & 0.0008 & 0.0017 \\
\hline Mass-Averaged Energy Loss Coefficient, $\boldsymbol{\zeta}$ & 0.031 & 0.033 \\
\hline Profile Energy Loss Coefficient, $\boldsymbol{\zeta}_{\boldsymbol{P}}$ & 0.020 & 0.022 \\
\hline Secondary Energy Loss Coefficient, $\boldsymbol{\zeta}_{\boldsymbol{S}}$ & 0.011 & 0.011 \\
\hline
\end{tabular}




\subsection{Results from the High-Turning Cascades: SL1F and SL2F}

\subsubsection{Turbine Cascades}

The high-turning turbine cascades are referred to as SL1F and SL2F: SL2F is more highly-loaded than SL1F. Table 7.4 summarizes the geometric and aerodynamic parameters of the turbine cascades at both design and off-design conditions. The results from the high-turning cascades are also compared with the results from the low-turning cascades in the next sections: SL1F and SL3F have similar aerodynamic loading and are referred to as "baseline loaded", whereas SL2F and SL4F are "more highly loaded". Whereas SL3F and SL4F were tested at only the design outlet Mach number, SL1F and SL2F were tested at two additional outlet Mach numbers.

Table 7.4 Summary of cascade parameters: high-turning airfoils

\begin{tabular}{|l|c|c|c|c|c|c|c|}
\hline & $\boldsymbol{C}(\mathbf{m m})$ & $\boldsymbol{C}_{\boldsymbol{X}}(\mathbf{m m})$ & $\boldsymbol{h}(\mathbf{m m})$ & $\boldsymbol{O}(\mathbf{m m})$ & $\boldsymbol{s}(\mathbf{m m})$ & $\boldsymbol{t}_{\max }(\mathbf{m m})$ & $\boldsymbol{S}\left(^{\circ}\right)$ \\
\hline SL1F & 31.0 & 25.4 & 61.0 & 9.5 & 26.8 & 7.9 & 34.9 \\
\hline SL2F & 33.7 & 25.4 & 61.0 & 12.0 & 34.0 & 7.5 & 41.2 \\
\hline
\end{tabular}

\begin{tabular}{|c|c|c|c|c|c|c|c|c|c|c|c|}
\hline & $\boldsymbol{M}_{\mathbf{1}}$ & $\boldsymbol{M}_{\mathbf{2}, \mathbf{s}}$ & $\boldsymbol{M}_{\mathbf{2}}$ & $\boldsymbol{\beta}_{\mathbf{1}}\left({ }^{\circ}\right)$ & $\boldsymbol{\beta}_{\mathbf{2}}\left({ }^{\circ}\right)$ & $\mathbf{Z w}$ & $\boldsymbol{A V D R}$ & $\boldsymbol{A V R}$ & $\boldsymbol{R V R}$ & $\boldsymbol{R e}_{c, 1}$ & $\boldsymbol{R e}_{c, 2}$ \\
\hline \multirow{3}{*}{ SL1F } & 0.265 & 0.65 & 0.64 & 43.5 & 68.9 & 0.81 & 0.98 & 1.16 & 2.34 & 230,000 & 480,000 \\
\cline { 2 - 12 } & 0.286 & 0.78 & 0.76 & 43.5 & 69.1 & 0.75 & 0.98 & 1.25 & 2.54 & 270,000 & 580,000 \\
\cline { 2 - 12 } & 0.294 & 0.91 & 0.87 & 43.5 & 69.3 & 0.66 & 0.99 & 1.36 & 2.78 & 310,000 & 680,000 \\
\hline \multirow{3}{*}{ SL2F } & 0.263 & 0.65 & 0.64 & 43.5 & 67.9 & 1.07 & 1.02 & 1.22 & 2.36 & 240,000 & 500,000 \\
\cline { 2 - 11 } & 0.283 & 0.78 & 0.76 & 43.5 & 68.2 & 0.97 & 1.02 & 1.31 & 2.56 & 280,000 & 600,000 \\
\cline { 2 - 10 } & 0.291 & 0.91 & 0.89 & 43.5 & 68.6 & 0.89 & 1.02 & 1.44 & 2.87 & 310,000 & 690,000 \\
\hline
\end{tabular}

\subsubsection{Midspan Blade Loading Distributions}

The midspan surface Mach number distributions are presented in Figure 7.10. The $30 \%$ increase in Zweifel coefficient for SL2F has been achieved by increasing the blade spacing. SL2F is also more mid-loaded than SL3F. Increasing the outlet Mach number results in smaller Zweifel coefficients, higher row velocity ratios and higher Reynolds numbers, as presented in Table 7.4. The total flow turning and the axial velocity density ratio, however, remain approximately constant. Raising the Mach number also results in 
more aft-loaded midspan loading distributions, as shown in Figure 7.11, and slightly smaller profile losses (Chapter 6). These results regarding the effects of Mach number on loading distributions are consistent with the findings by Camus et al. (1984), Duden and Fottner (1997) and Dossena et al. (1997).
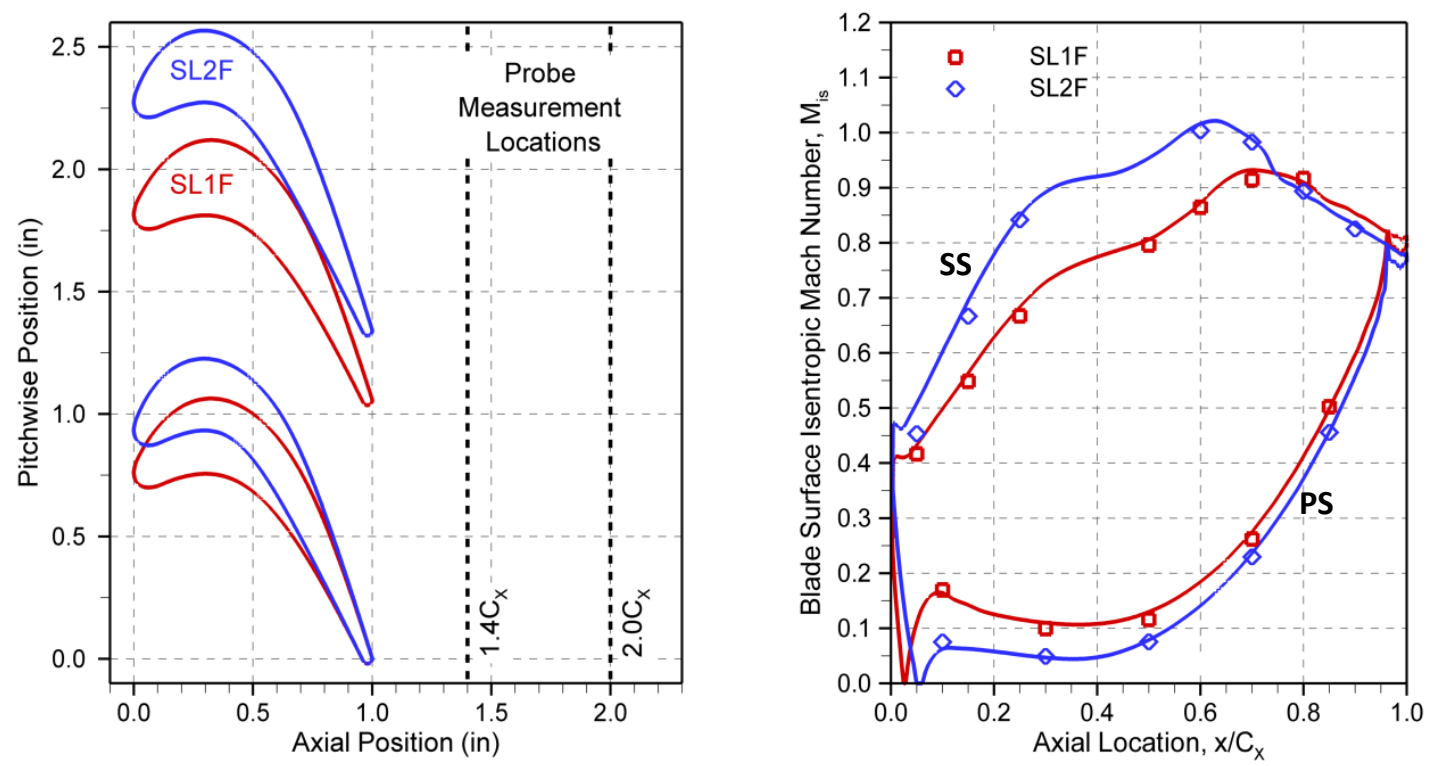

Figure 7.10 High-turning airfoils: midspan loading distributions at design conditions
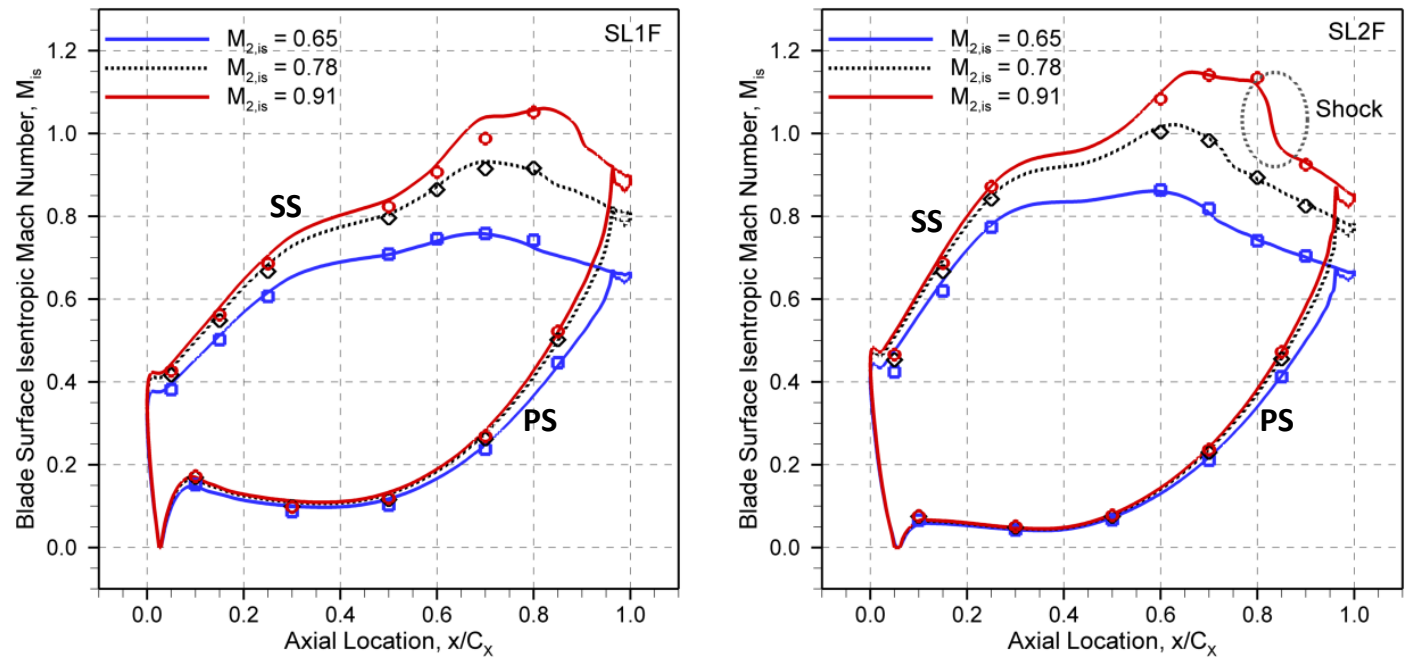

Figure 7.11 High-turning airfoils: midspan loading distributions at off-design conditions 


\subsubsection{Surface Flow Visualization Results at Design Conditions}

The surface flow visualization studies were conducted for the high-turning airfoils at the design Mach number using a mixture of fluorescent-dye and motor oil, illuminated and photographed under ultra-violet lighting following the completion of the tests; the results are presented in Figure 7.12 and Figure 7.13. The nomenclature of Sieverding (1985) is used to interpret the limiting streamlines: dashed lines are used for flow separation, dotted lines for flow reattachment and arrows for the surface shear stress vectors.

- $\quad \mathrm{A}_{1}$ : saddle point of inlet boundary layer separation

- $\mathrm{S}_{1}$ : primary separation line of inlet boundary layer

- $\mathrm{S}_{2}$ : liftoff line of the horseshoe vortex

- $\quad \mathrm{S}_{3}$ : suction side corner vortex separation

- $\mathrm{S}_{4}$ : passage vortex separation

- $\mathrm{S}_{5}$ : suction-surface profile flow separation

- $\mathrm{R}_{5}$ : profile flow reattachment

- $\quad \mathrm{S}_{6}$ : trailing edge separation

- $\mathrm{R}_{2}$ : reattachment at the blade/endwall junction

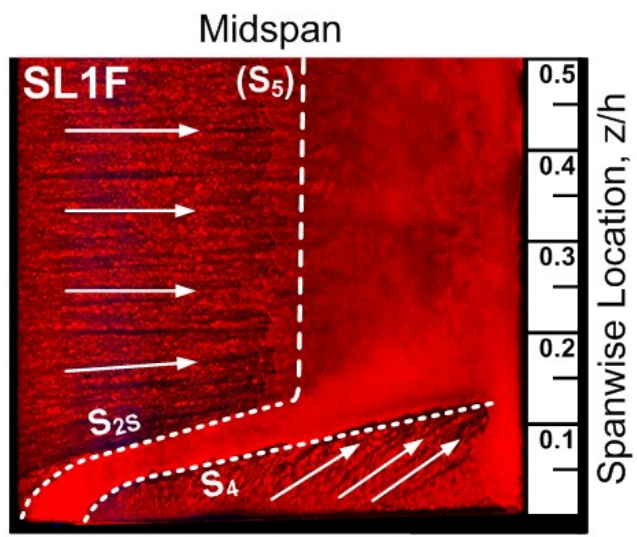

Endwall

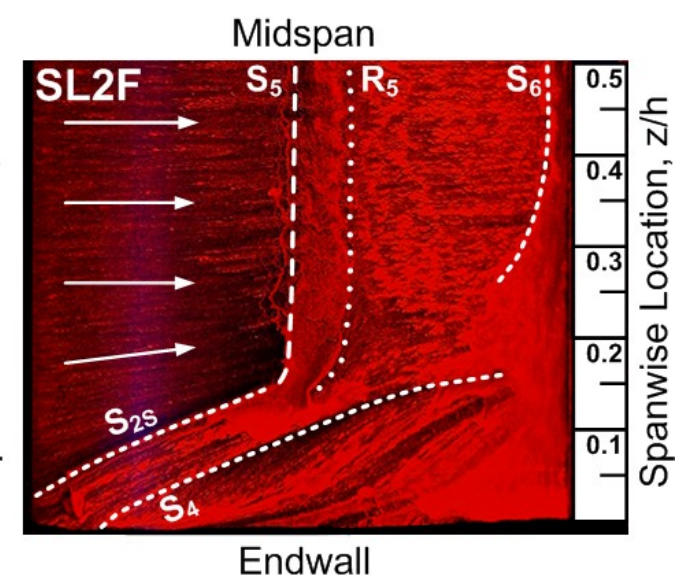

Endwall

Figure 7.12 Surface flow visualization results $\left(M_{2, i s}=0.78\right)$ : airfoil suction surfaces

As shown in Chapter 6, the transitional CFD simulations predict fully attached suction-surface boundary layers at midspan at the design Mach number. The blade surface visualization results in Figure 7.12, on the other hand, show evidence of suction side separation $\left(\mathrm{S}_{5}\right)$ on both airfoils. In spite of the overall loading difference, which 
would have been expected to results in higher profile losses for the more highly loaded SL2 cascade, SL1 and SL2 in fact produce similar profile losses, possibly as a result of the separation bubbles.
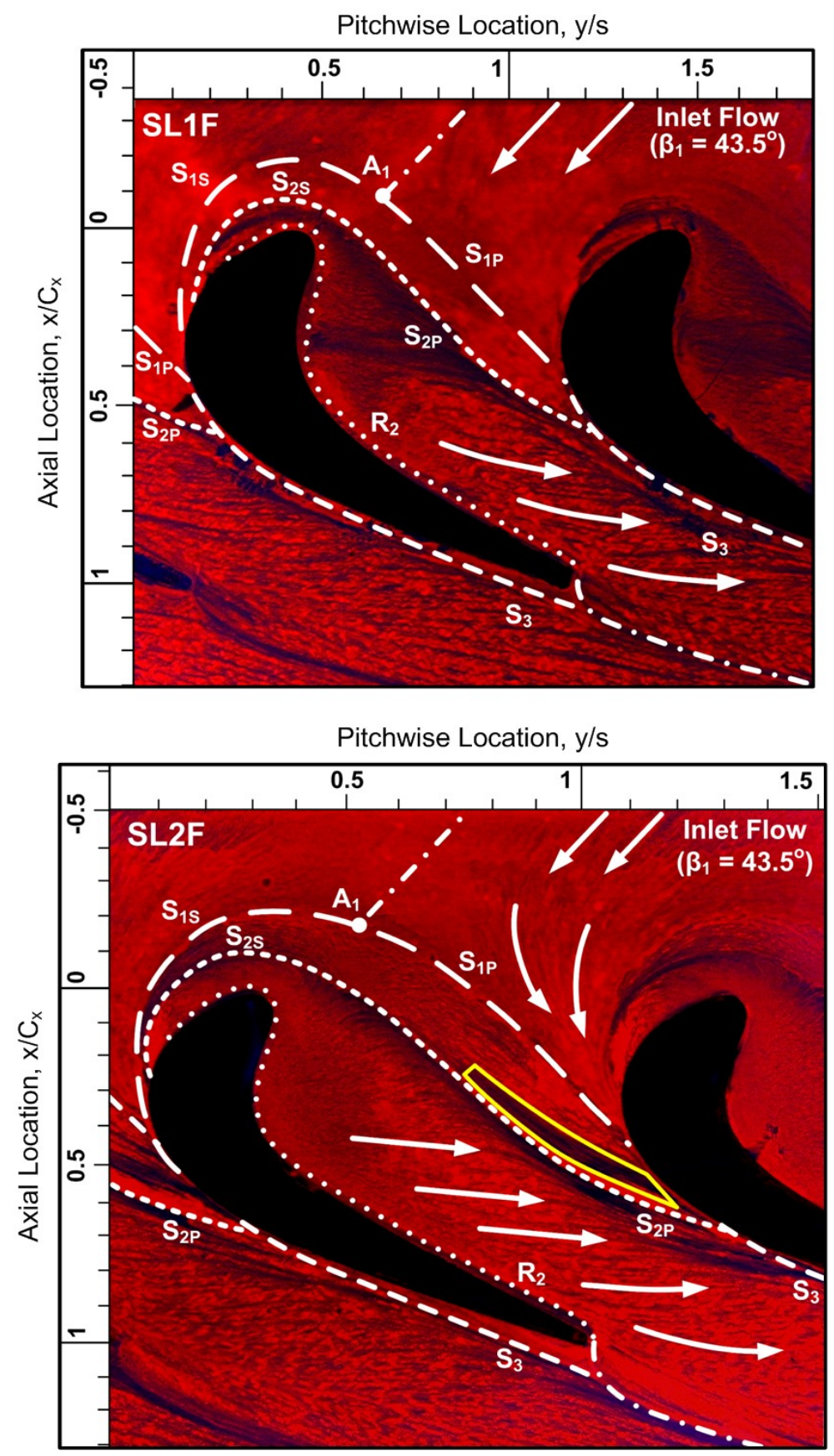

Figure 7.13 Surface flow visualization results $\left(M_{2, i s}=0.78\right)$ : endwalls

In Figure 7.13, the saddle point for inlet boundary layer separation $\left(A_{1}\right)$ is located farther upstream for SL2F than for SL1F, as a result of the stronger transverse pressure 
difference in the forward part of the passage (Figure 7.10). Furthermore, the extent of the near-stagnation conditions on the forward part of the airfoil determines the size of the apparent obstacle that the endwall boundary layer sees, and as shown in Figure 7.10, SL2F displays lower velocities in this region. Accordingly, the primary separation line $\left(\mathrm{S}_{1}\right)$ also extends slightly farther upstream for SL2F. $\mathrm{S}_{2}$ marks the liftoff line of the horseshoe vortex ahead of the leading edge plane. Under the action of the cross-passage pressure gradient, the pressure-side leg of the horseshoe vortex, marked by $\mathrm{S}_{2 \mathrm{P}}$, migrates towards the suction surface of the adjacent blade. In this process, the pressure-side leg of the horseshoe vortex entrains low momentum fluid from the endwall boundary layer, and merges with the passage vortex. Once $S_{2 P}$ encounters the airfoil suction surface, the passage vortex migrates away from the endwall as indicated by $S_{4}$ in Figure 7.12 towards the midspan. The passage vortex is also subjected to an adverse streamwise pressure gradient following the suction peak inside the passage. As judged by the $\mathrm{S}_{4}$ lines in Figure 7.12, SL2F displays higher secondary flow spanwise penetration than SL1F.

In Figure 7.13, the suction-side leg of the horseshoe vortex, marked by $\mathrm{S}_{2 \mathrm{~S}}$, is also directed towards the blade surface by the cross-passage pressure gradients. As shown in Figure $7.12, \mathrm{~S}_{2 \mathrm{~S}}$ seems to vanish as it encounters profile flow separation $\left(\mathrm{S}_{5}\right)$. It is typically assumed that the suction-side leg of the horseshoe vortex becomes part of the passage vortex (possibly wrapped around the passage vortex) since it does not appear separately in the downstream vorticity plots (probe results in Section 7.4.4). The region between $S_{2 S}$ and $S_{4}$ in Figure 7.12 is believed to contain inlet boundary layer fluid, convected through the passage, which is subsequently revealed as a loss core within the counter vortex downstream of the cascade. In Figure 7.12, SL1F shows significantly smaller shear stresses in this region than SL2F, potentially resulting in higher mixing in the free shear layer by the passage vortex.

SL1F shows generally higher endwall surface shear stresses within the blade rows than SL2F. This is particularly evident between $S_{2}$ and $R_{2}$ in the forward part of the passage $\left(-0.1<x / C_{X}<0.5\right)$, and is possibly due to the closer proximity of the passage vortex to the endwall for SL1F (consistent with lower secondary flow penetration). The SL2F results, on the other hand, display a region of high surface shear stress upstream of $\mathrm{S}_{2 \mathrm{P}}$ (very low pigment region enclosed by the yellow line), which does not appear in 
SL1F. Based on the current data sets, it is not possible to identify the specific flow feature responsible for the higher surface shear stresses in SL2F. This region is typically associated with "weak" boundary layer convergence in low-speed studies (Benner, 2003).

In Figure 7.13 , the corner vortex separation lines $\left(\mathrm{S}_{3}\right)$ are visible in both cascades, although more pronounced for SL2F. The corner vortices, however, seem to originate at slightly different axial locations. For SL1F, $\mathrm{S}_{3}$ originates at the intersection of $\mathrm{S}_{1 \mathrm{P}}$ with the airfoil suction surface $\left(x / C_{X} \approx 0.49\right)$, which may be regarded as "strong interaction" (identified by the large incidence angle between the endwall limiting streamline and the blade surface (Sieverding, 1984)). For SL2F, the corner vortex seems to originate just upstream of $\mathrm{S}_{2 \mathrm{P}}\left(x / C_{X} \approx 0.53\right)$ in a region of low surface shear stress. The corner vortex is intensified in the aft-part of the passage as it entrains boundary layer fluid from the endwall, as shown by the direction of the surface shear stress vectors (arrows in Figure 7.13). SL2F displays a larger corner vortex as indicated by the larger distance between $\mathrm{S}_{3}$ and the suction surface in Figure 7.13. This region also shows smaller surface shear stresses for SL2F, suggesting a larger distance between the vortex and the endwall. In Figure 7.13, the corner vortices are displaced away from the suction surfaces downstream of the cascades, possibly due to trailing edge separation; this feature was also evident in Detemple-Laake's (1990) flow visualization results. The vortical structures are discussed in more detail in the next section with reference to downstream probe measurements.

\subsubsection{Downstream Flow Field Measurements at $1.4 C_{X}$}

Figure 7.14(a) shows colour flood contour plots of local axial velocity density ratio $\left(A V D R_{\text {local }}\right)$, superimposed over contour lines of energy loss coefficient $(\zeta)$ at the design outlet Mach number of 0.78. The higher Zweifel coefficient for SL2F was achieved by increasing the blade spacing, which is also evident in Figure 7.14(a). The pitchwise mass-averaged energy loss coefficients $\left(\zeta^{\prime}\right)$ are shown in Figure 7.14(b).

The contour lines of energy loss coefficient in Figure 7.14(a) demonstrate higher spanwise secondary flow penetration for SL2F than for SL1F as indicated by the spanwise extent of secondary losses (confirming the flow visualizations results in Figure 7.12). SL2F displays three distinct secondary loss cores in Figure 7.14(a): these are associated with the corner vortex (near the endwall at approximately $90 \%$ pitch), the 
(a)

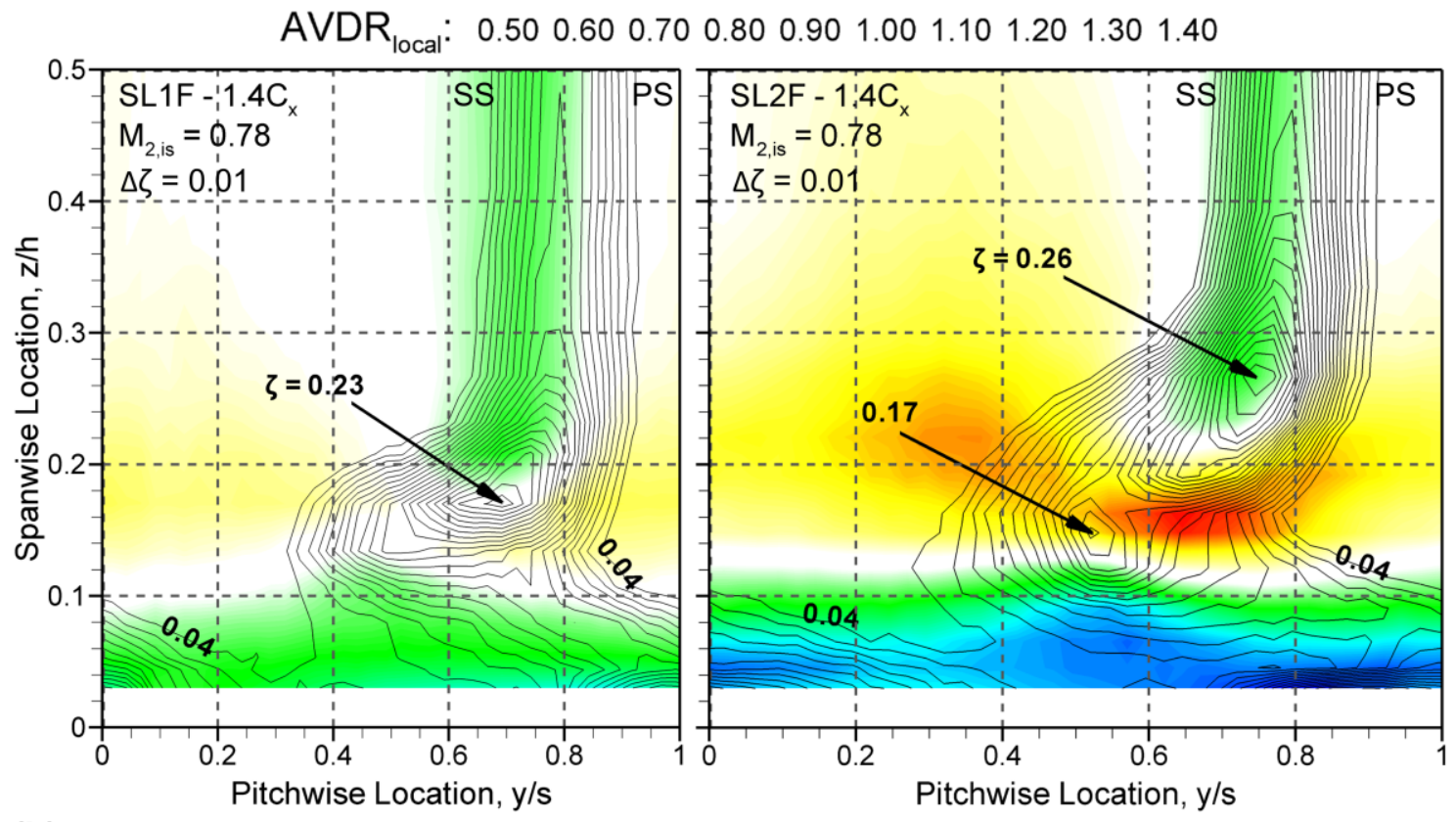

(b)

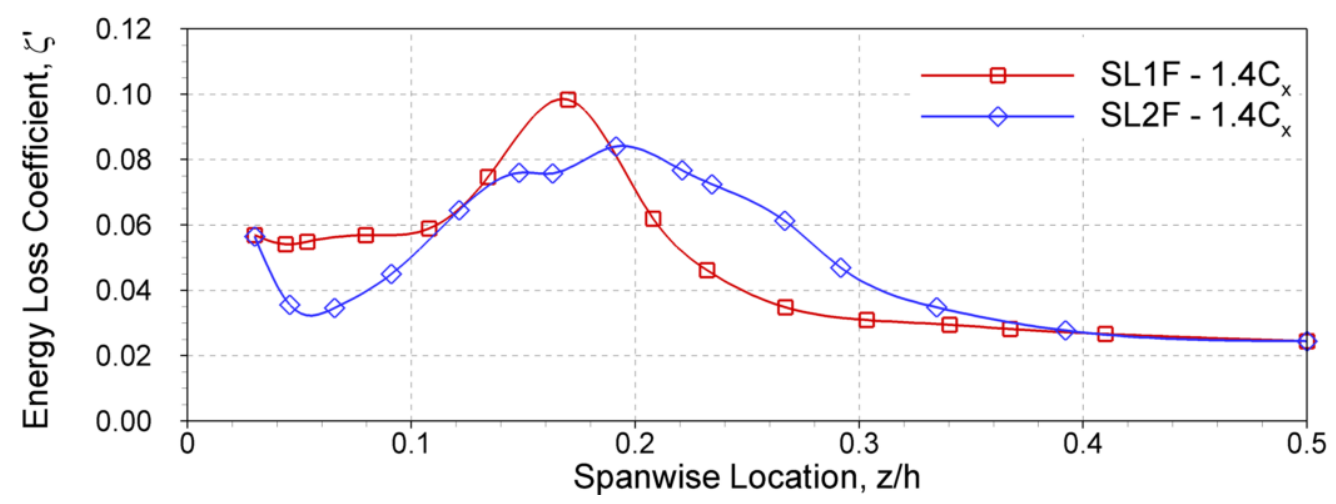

Figure 7.14 (a) Colour floods of local axial velocity density ratio superimposed over contour lines of energy loss coefficient, and (b) pitch-averaged energy loss coefficients $\left(M_{2}=0.78\right)$

passage vortex (at $\zeta \approx 0.17$ ) and the counter vortex (at $\zeta \approx 0.26$ ). These vortices are identified in the streamwise vorticity plots in Figure 7.15. SL1F, on the other hand, shows only two visible loss cores: one located near the endwall associated with the corner vortex, and a second at approximately $17 \% \operatorname{span}($ at $\zeta \approx 0.23$ ) within the counter vortex. The lossy fluid associated with the passage vortex does not result in a distinct loss core for SL1F. In both cascades, the peak losses coincide roughly with the position of the counter vortices. As discussed with reference to the flow visualization results in Figure 7.12, part of the inlet boundary layer fluid that is convected between $S_{4}$ and $S_{2 S}$ through the blade row is subsequently swept up the suction surface and becomes part of the 
counter vortex downstream of the cascade. Consequently, the peak total pressure deficits in Figure 7.14(a) $(\zeta=0.23$ in SL1F and 0.26 in SL2F) are partially comprised of inlet boundary layer losses, which do not contribute to the overall-integrated row losses. The losses associated with the corner vortices, in contrast, are predominantly generated in the blade passages in the newly-formed transitional endwall boundary layers (downstream of $\mathrm{S}_{2}$ in Figure 7.13).

In Figure 7.14(a), weighting the total pressure losses by $A V D R_{\text {local }}$, which is representative of the mass-averaging procedure, results in larger contributions to the losses in regions of high mass flux, from $15 \%$ to $25 \%$ span for SL2F, and smaller contributions in regions of low mass flux, from 3\% to $10 \%$ span for SL2F. SL2F displays larger variations in $A V D R_{\text {local }}$ due to stronger vortical structures, as shown in Figure 7.15. The pitch-averaged results in Figure 7.14(b) show similar levels of profile losses in both cascades. SL2F, however, displays higher secondary flow penetration distance than SL1F, as already seen from the contour plots, and accordingly it produces higher losses from $20 \%$ to $35 \%$ span. SL1F, on the other hand, generates higher massaveraged losses from $3 \%$ to $10 \%$ span due to relatively higher mass fluxes in this region $\left(A V D R_{\text {local }} \approx 0.8\right)$ than for $\mathrm{SL2F}\left(A V D R_{\text {local }} \approx 0.6\right)$. Despite the significant loss redistribution in Figure 7.14(a), the two cascades produce similar mass-averaged row losses (within $2 \%$ ) at $1.4 C_{X}$.

Figure 7.15 shows colour floods of streamwise vorticity coefficient $\left(C_{\omega s}\right)$ superimposed over contour lines of energy loss coefficient for three different outlet isentropic Mach numbers: 0.65, 0.78 and 0.91. The passage vortex has positive vorticity, whereas the counter and corner vortices have negative vorticity. In Figure 7.15, the contour lines of energy loss coefficient have been distorted in the secondary flow region under the influence of the vortices. SL2F exhibits stronger vortical structures than SL1F at all Mach numbers; the passage vortex, in particular, is significantly stronger for SL2F. The passage vortex and the counter vortex are slightly closer to midspan for SL2F, which is consistent with the higher secondary flow penetration in Figure 7.12. Furthermore, SL2F shows a larger and more intense corner vortex, as anticipated from the endwall flow visualization results. 


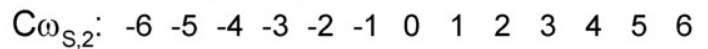
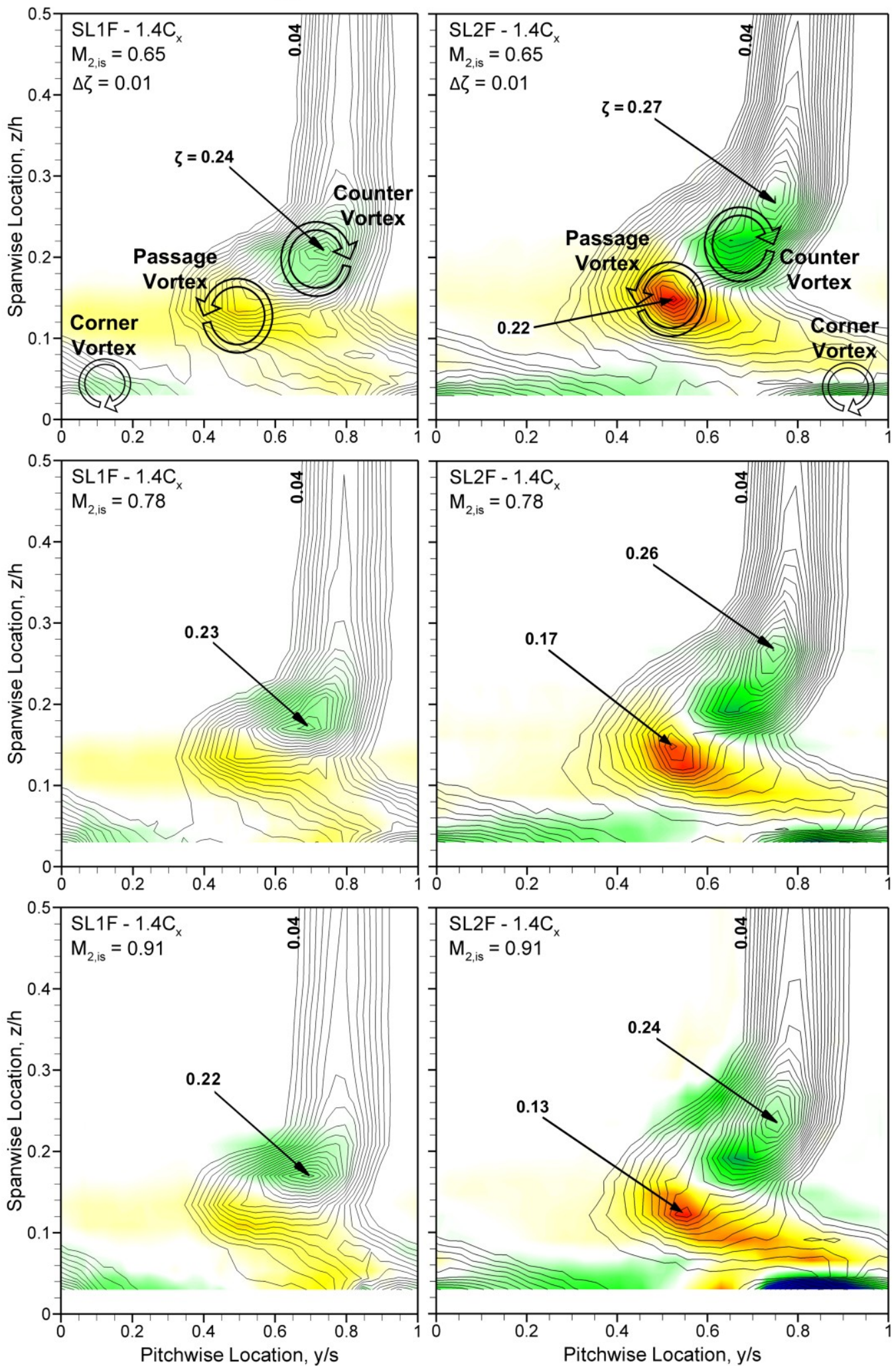

Figure 7.15 Streamwise vorticity coefficients $\left(C_{\omega s}\right)$ at three different exit Mach numbers 
As discussed in Section 7.4.2, the results from the additional Mach numbers not only represent the effects of flow compressibility, but also represent other off-design conditions. The vorticity plots in Figure 7.15 show smaller streamwise vorticity coefficients associated with the passage and the counter vortices at higher Mach numbers. The smaller vorticity coefficients are attributed in part to the higher row velocity ratios: raising the expansion ratio is believed to delay the development of the vortices inside the blade row. The vortices are also located closer to the endwall at higher Mach numbers, which is consistent with the finding by Perdichizzi (1990). Accordingly, the contour lines of energy loss coefficient display slightly lower secondary flow penetration at higher Mach numbers, as shown by the spanwise extent of secondary losses in Figure 7.15. Raising the Mach number is accompanied by a reduction in secondary losses: smaller peak energy loss coefficients and smaller secondary flow blockage (the portion of the measurement plane containing secondary flow).

Figure 7.16 shows colour floods of secondary kinetic energy coefficient $\left(C_{S K E}\right)$, overlaid on secondary velocity vectors for the three outlet Mach numbers. There are two distinct areas of high secondary kinetic energy: the largest values of $C_{S K E}$ are found in the interaction region between the passage vortex and the counter vortex (14\% to $22 \%$ of span). Closer to the endwall, below 10\% span for SL2F, large pitchwise secondary velocities are induced by the action of the passage vortex, resulting in flow overturning. The $C_{S K E}$ contour plots at the design Mach number exhibit strong associations with the mass flux distributions in Figure 7.14(a), both in terms of location and magnitude.

The magnitudes of the secondary velocity vectors are directly proportional to the size and peak vorticity of the vortical structures; raising the outlet Mach number results in smaller vorticity coefficients in Figure 7.15 and smaller secondary velocities in Figure 7.16. Consequently, in both cascades, the secondary kinetic energy coefficients are smaller at higher Mach numbers. As shown in Figure 7.16, SL2F produces significantly higher $C_{S K E}$ than SL1F at all three Mach numbers; the higher SKE production for SL2F is associated with the stronger vortical structures (Figure 7.15). At the design Mach number, the largest secondary velocities for SL2F (at $z / h \approx 0.15$ ) are $20 \%$ of the value of the primary velocity $\left(U_{2, i s}\right)$ as opposed to $10 \%$ for SL1F. SL2F also displays larger secondary velocities than SL4F (Figure 7.7), possibly due to the higher flow turning. 

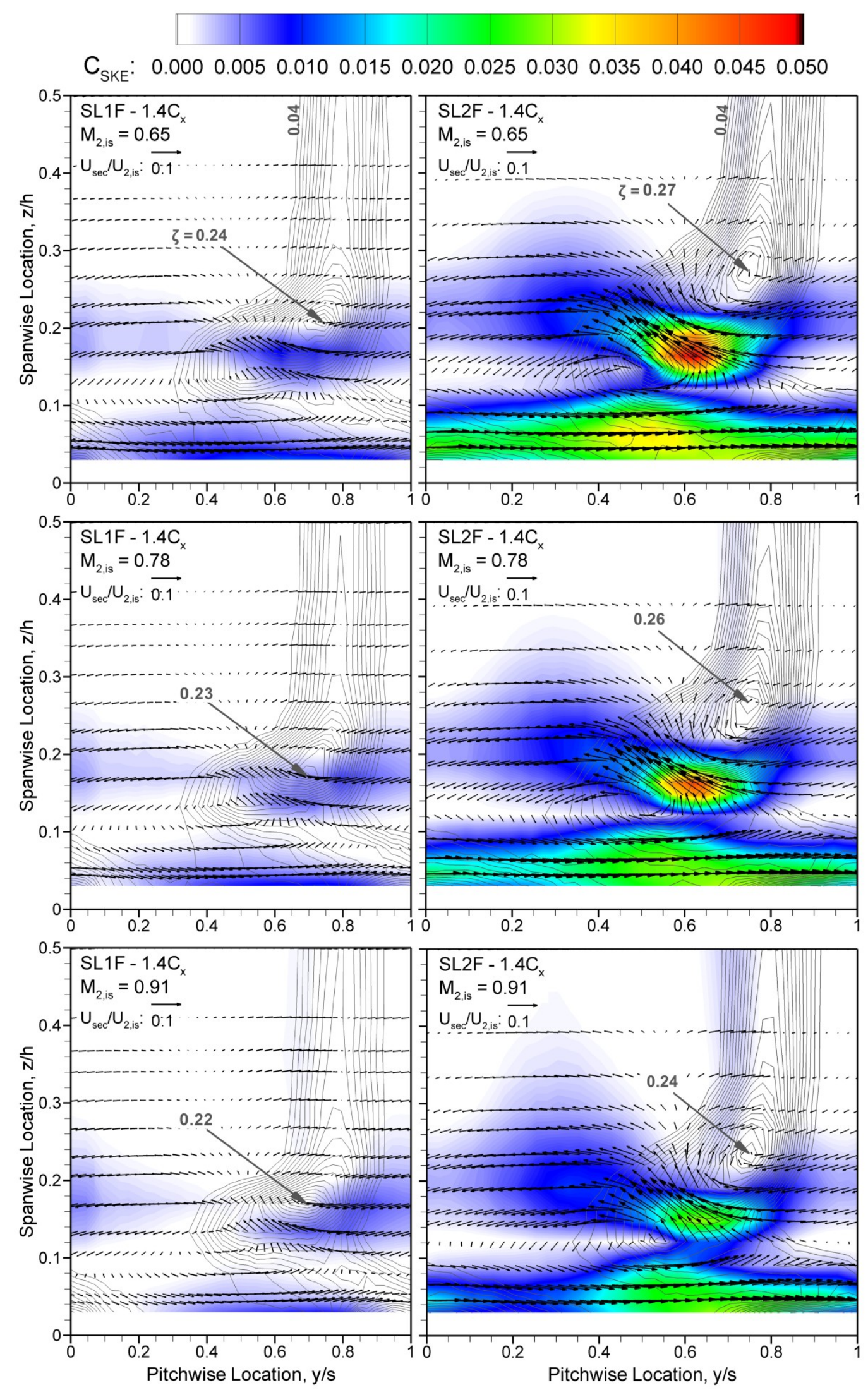

Figure 7.16 Secondary kinetic energy coefficients at three different exit Mach numbers 
In low-speed studies (Moore and Adhye, 1985; Gregory-Smith et al., 1988a; Benner et al., 2004), the mixing out of the secondary kinetic energy downstream of the cascades is often the dominant loss production mechanism as the flow approaches fully mixed-out conditions. Accordingly, SL2F is expected to generate higher losses downstream of $1.4 C_{X}$ than SL1F, due to the higher $C_{S K E}$ in Figure 7.16. The downstream growths of the secondary losses are discussed in the subsequent sections with reference to the probe measurements at $2.0 C_{X}$.

\subsubsection{Pitchwise Mass-Averaged Results at $1.4 C_{X}$}

Figure 7.17 shows the pitch-averaged results at $1.4 C_{X}$ : the energy loss coefficients, which include the total pressure deficit already present in the inlet endwall boundary layer, the secondary kinetic energy coefficients, and the exit flow angles. The secondary kinetic energy coefficients have been defined based on the midspan (primary) flow direction, and therefore are negligible in the profile flow region.

Figure 7.17(a) shows slight reductions in profile losses at higher Mach numbers. As shown in Chapter 4, the energy loss coefficient is defined based on the outlet isentropic Mach number. Consequently, the profile loss coefficients in Figure 7.17(a) are affected not only by the absolute entropy generation within the blade row, but also by the exit Mach numbers. This non-dimensionalization method is consistent with the definition of the Zweifel coefficient, also defined based on the isentropic outlet dynamic pressure: as the exit Mach number is raised, the Zweifel coefficients decrease (Table 7.4), so the slight reductions in profile losses at higher Mach numbers correlate with the smaller blade loading coefficients. The secondary losses in Figure 7.17(a) decrease significantly at higher Mach numbers, as discussed with reference to the vorticity plots in Figure 7.15. The spanwise extents of secondary losses also decrease at higher Mach numbers. It is worth noting that increasing the outlet Mach number for SL2F does not significantly influence the near-wall energy loss coefficients from $3 \%$ to $10 \%$ span. This is attributed to the intensification of the corner vortex at higher exit Mach numbers (Figure 7.15), resulting in larger mass fluxes near the endwalls and thus larger contributions to the mass-averaged losses (the resulting loss coefficients in this region are nearly unchanged since they are non-dimensionlized based on $M_{2, i s}$ ). Similar trends appear in the near-wall 
region for SL1F, albeit less pronounced due to a weaker corner vortex. These results are consistent with the findings of Duden and Fottner (1997).
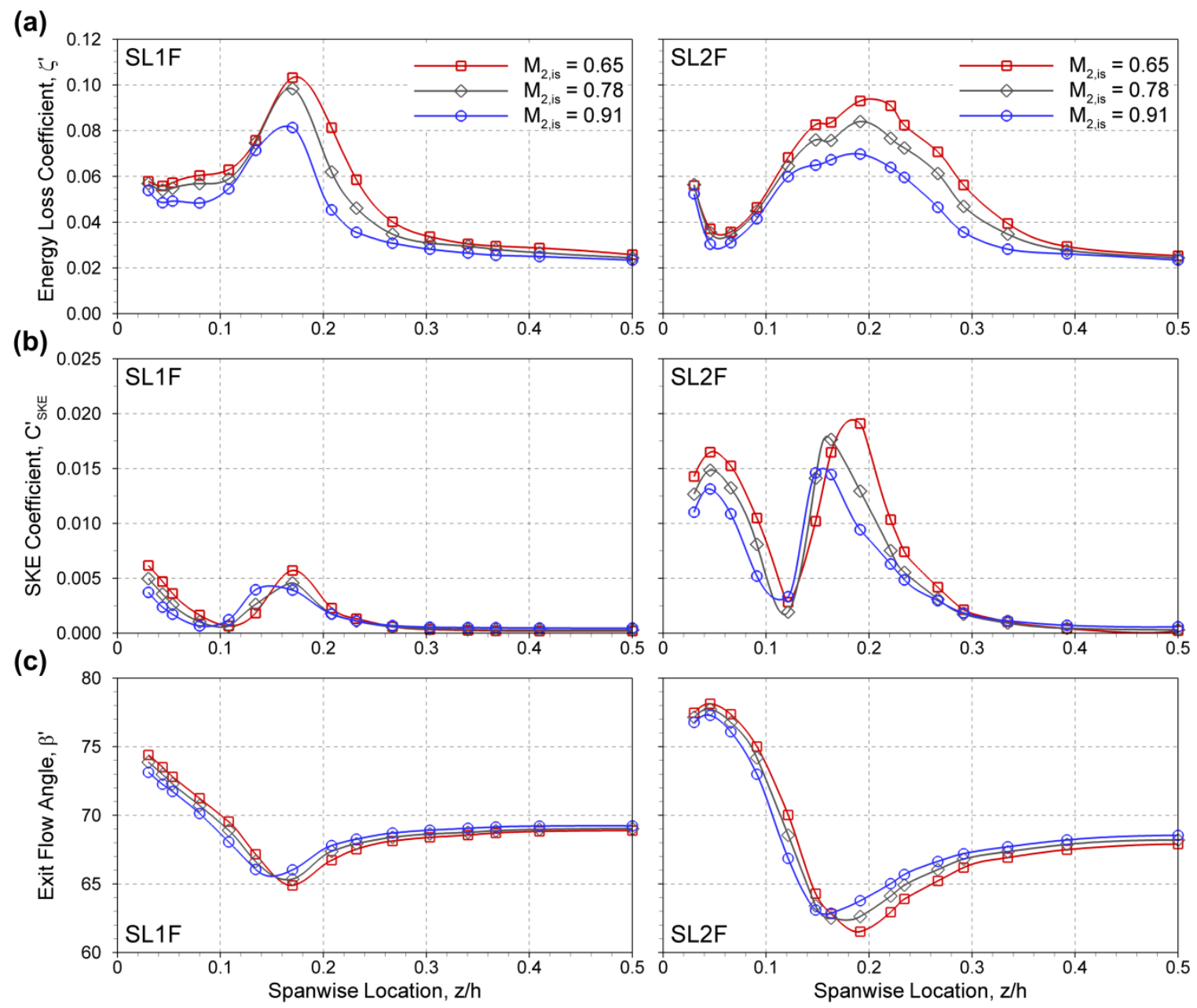

Figure 7.17 Pitch-averaged results at $1.4 C_{X}$ : (a) energy loss coefficients, (b) secondary kinetic energy coefficients and (c) exit flow angles

Figure 7.17(b) shows significantly higher secondary kinetic energy production for the same downstream location for SL2F than for SL1F at all three Mach numbers. Furthermore, as expected from the contour plots in Figure 7.16, both airfoils produce smaller $C_{S K E}^{\prime}$ at higher exit Mach numbers due to smaller secondary velocities. The peaks of $C_{S K E}^{\prime}$ in Figure 7.17(b) associated with passage/counter vortex interaction (at $10 \%$ to $20 \%$ span) migrate closer to the endwall at higher Mach numbers. The peaks of $C_{S K E}^{\prime}$ associated with the corner vortex, on the other hand, do not exhibit any spanwise displacement near the endwalls. 
The exit flow angles in Figure 7.17(c) depict the classic underturning/overturning characteristic associated with secondary flows. The peaks of $C_{S K E}^{\prime}$ in Figure 7.17(b) are aligned with the positions of the maximum underturning and overturning in Figure 7.17(c). Overall, SL2F displays larger variations in the exit flow angles than SL1F due to stronger vortical structures. Closer to the endwall for SL2F, from 3\% to 5\% span, flow overturning is slightly reduced under the action of the counter-rotating corner vortex. In

a gas turbine, the larger exit flow angle variations downstream of SL2F would adversely affect the performance of the subsequent blade rows due to off-design incidence effects. Increasing the outlet Mach number results in smaller exit flow angle variations as the primary flow assumes a more important role. Perdichizzi (1990) and Dossena et al. (2004) found similar trends in underturning and overturning reductions at higher Mach numbers, including supersonic conditions.

\subsubsection{Downstream Growth of Secondary Losses at the Design Mach Number}

As identified in Figure 7.10, traverse measurements were made for SL1 and SL2 at the design Mach number at an additional downstream plane at 2.0 $C_{X}$. Figure 7.18(a) shows colour floods of secondary kinetic energy coefficient, overlaid on secondary velocity vectors at $2.0 C_{X}$. The corresponding pitchwise mass-averaged results are presented in Figure 7.18(b) and Figure 7.18(c).

Comparing the results in Figure 7.18(a) to the contour plots in Figure $7.16\left(M_{2, \text { is }}=\right.$ 0.78) reveals significant spreading of the secondary flows downstream of the cascades: larger secondary loss regions with smaller peak losses, and smaller secondary kinetic energy coefficients. Furthermore, the secondary flow structures migrate away from the endwalls downstream of the cascades, and the loss coefficient contour lines are distorted under the action of the vortices. The pitchwise mass-averaged results in Figure 7.18(c) demonstrate small increases in profile losses between $1.4 C_{X}$ and $2.0 C_{X}$. The secondary losses, on the other hand, increase substantially downstream of the cascades due to both primary and secondary kinetic energy dissipation, as well as changes in static pressure (e.g. static pressure recovery). 
(a)

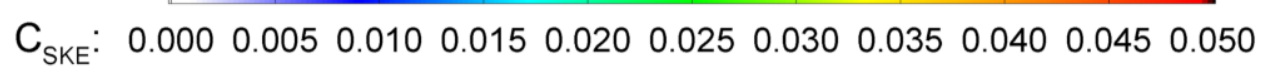
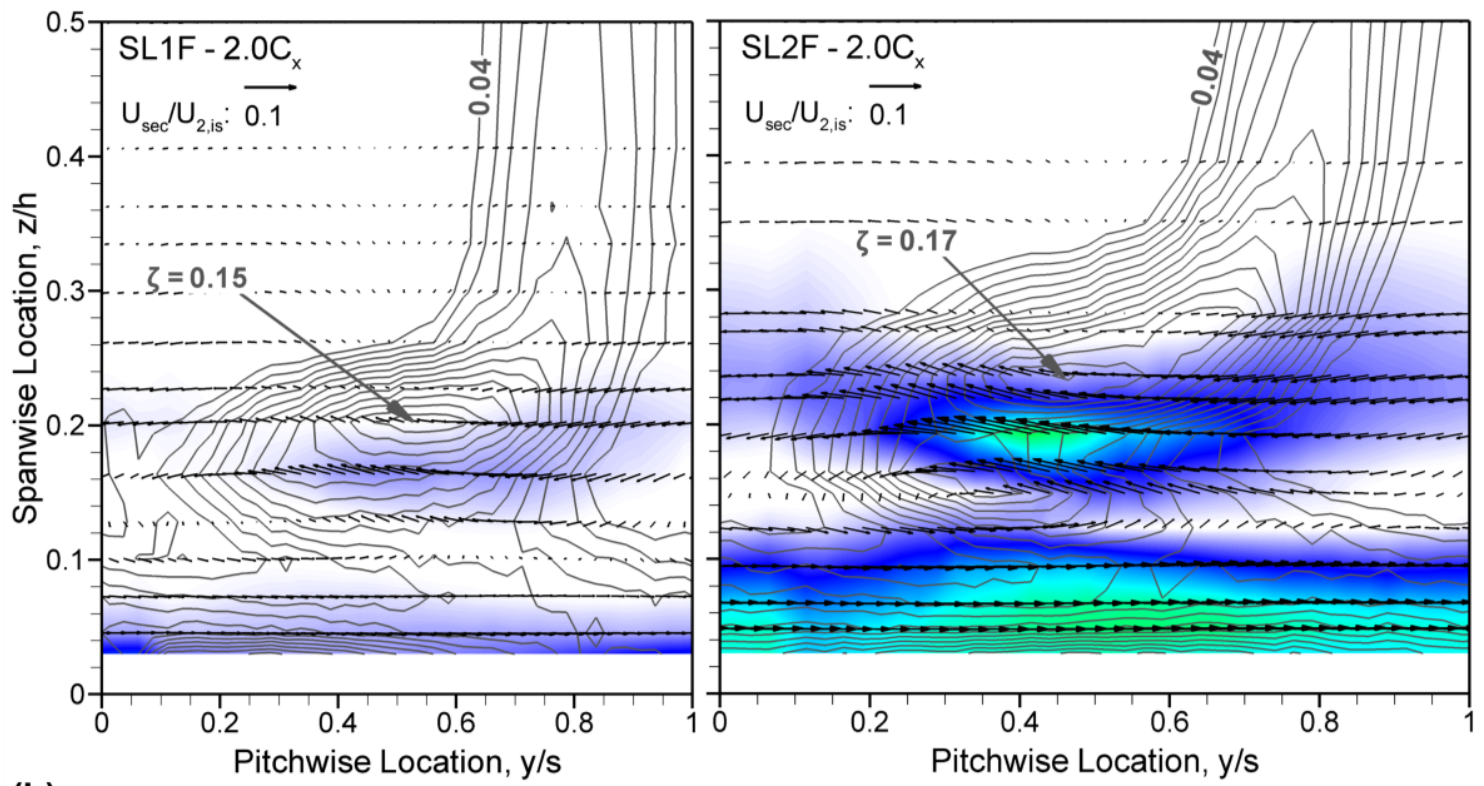

(b)

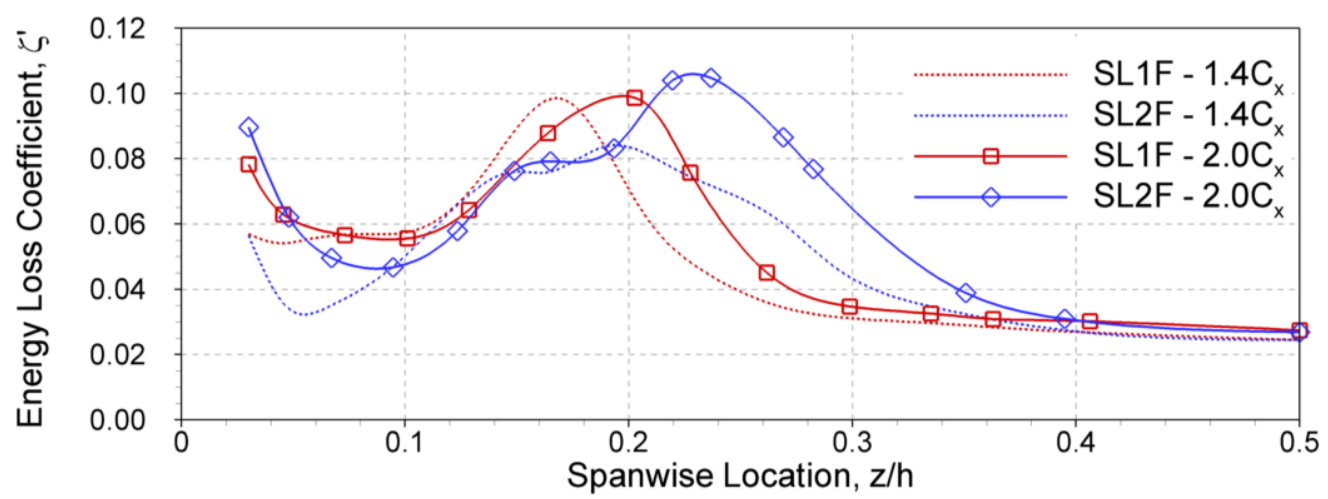

(c)

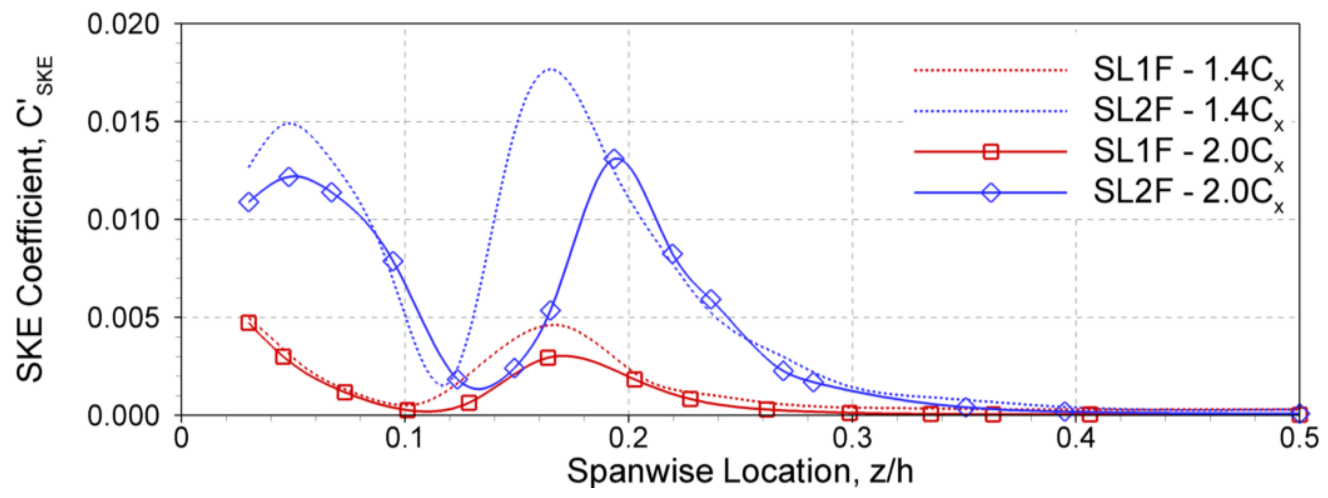

Figure 7.18 (a) Secondary kinetic energy coefficients at $2.0 C_{X}$, (b) pitch-averaged $C_{S K E}$ and (c) pitch-averaged energy loss coefficients $\left(M_{2, i s}=0.78\right)$

In Figure 7.15 and Figure 7.16, SL2F displays stronger vortical structures and significantly larger $C_{S K E}$ than SL1F at $1.4 C_{X}$. Consequently, SL2F produces higher secondary losses than SL1F downstream of $1.4 C_{X}$ (larger $S K E$ mixing out), leading to 
higher row losses at $2.0 C_{X}$. The decay of $S K E$, however, accounts for less than $15 \%$ of the additional secondary losses between the two measurement planes. Perdichizzi's (1990) measurements also showed significantly higher losses, from $1.1 C_{X}$ to $2.1 C_{X}$, than attributable to $S K E$ decay at high subsonic Mach numbers. These results suggest a more significant contribution from primary kinetic energy dissipation at higher Mach numbers, particularly in the near-wall regions due to higher free-stream velocities. Of course, these findings should be treated with some caution since the measurement planes only extend to $3 \%$ span in the current study; the treatment of the near-wall losses, from 0 to $3 \%$ span, was described in Chapter 4. In spite of the significant secondary flow mixing up to $2.0 C_{X}$, the SL2F results in Figure 7.18(c) show large $C_{S K E}^{\prime}$ below $10 \%$ span, associated with large pitchwise secondary velocities. Consequently, additional secondary losses are expected downstream of $2.0 C_{X}$ due to the mixing out of the remaining secondary kinetic energy. These results suggest that the more highly-loaded cascade, SL2F, ultimately will produce higher row losses than the baseline case, SL1F.

\subsubsection{CFD Predictions at the Design Mach Number}

The 3D CFD simulations were performed for SL1F and SL2F, based on the experimental boundary conditions at the design Mach number. As discussed in Chapter 5, the transition model in ANSYS CFX was not utilized for the 3D CFD simulations due to convergence problems. Therefore, the results in this section represent fully turbulent simulations at the design Mach number using the shear stress transport (SST) turbulence model.

The fully-turbulent simulations generally predict higher losses than the transitional simulations due to higher dissipation rates in turbulent boundary layers; turning off the transition model for SL2F results in $27 \%$ higher profile losses and larger skin friction coefficients (Chapter 6). Furthermore, both transitional and turbulent simulations predict larger profile losses than measured. The effects of the transition model on secondary losses are not documented in the present work due to the aforementioned convergence problems. The experimental results, which perhaps include both laminar and transitional boundary layers, may be utilized for compressible-flow CFD validation studies in the future. 
Figure 7.19 shows the predicted blade surface isentropic Mach numbers at four different spanwise locations: $5 \%, 10 \%, 25 \%$ and 50\% span. The inlet centreline total pressure $\left(P_{01, M S}\right)$ and the blade-surface static pressures were used to calculate the isentropic Mach numbers. The inlet total pressure, however, varies across the span due to the presence of inlet endwall boundary layers and the formation of the horseshoe vortex. Therefore, the isentropic Mach numbers at 5\% and 10\% span may be larger than the actual Mach numbers $\left(P_{0, \text { free-stream }}<P_{01, M S}\right)$. This effect is most pronounced near the leading edges, which may explain the apparent absence of stagnation points at $5 \%$ and $10 \%$ span.
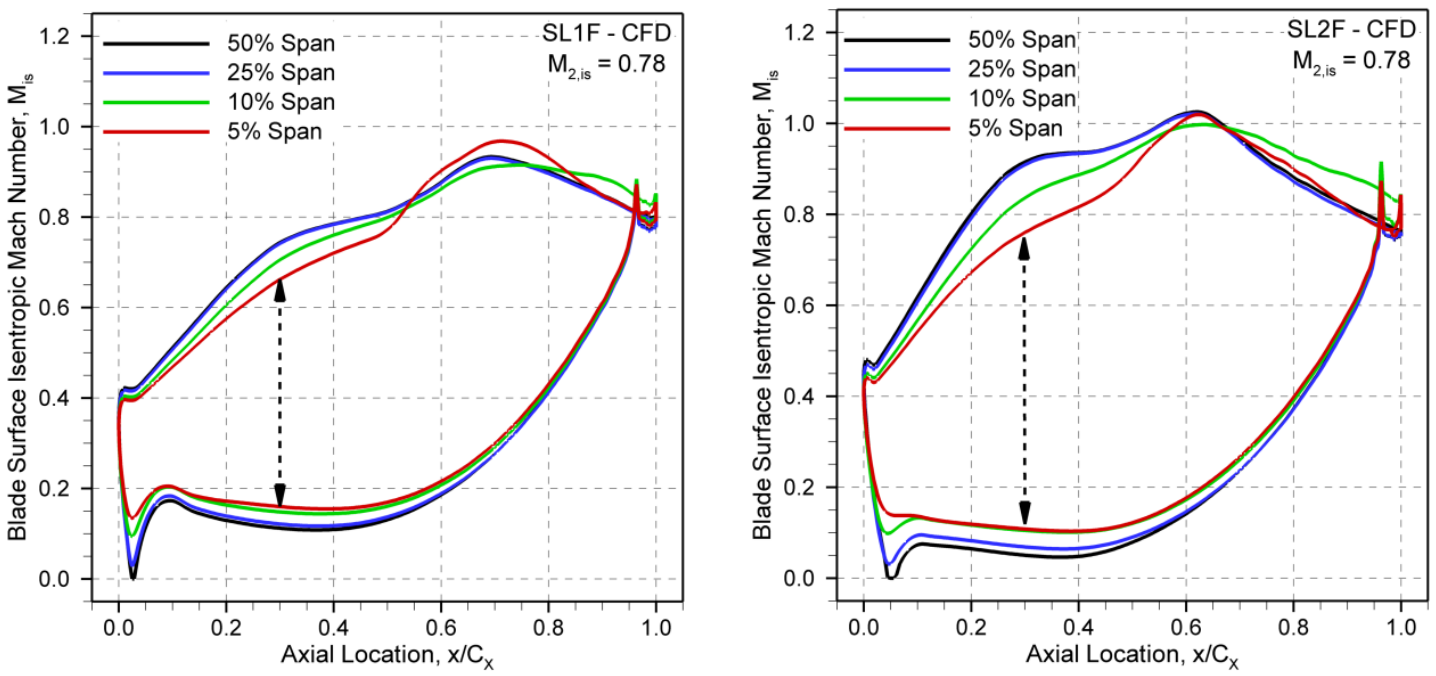

Figure 7.19 Blade loading distributions at various spanwise locations $\left(M_{2, i s}=0.78\right)$

SL1F and SL2F loading distributions display similar characteristics in Figure 7.19. In each case, the loading distribution at $25 \%$ span resembles the midspan loading distribution since the airfoil surface is not affected by the secondary flows at this spanwise location. At 10\% span, the passage vortex encounters the blade suction surface, resulting in lower static pressures on the aft part of the surface. Accordingly, the surface isentropic Mach numbers increase and the airfoils become more aft-loaded (green lines). The reduction in loading in the forward part of the airfoil continues at 5\% span (red lines), as shown by the arrows at $0.3 x / C_{X}$. Approaching the trailing edge, the loading distribution at 5\% span resembles the midspan loading distribution since this area resides below the passage vortex. Overall, the near-wall loading distributions display smaller 
cross-passage pressure gradients than suggested by Figure 7.10. This is typically referred to as endwall unloading (Harrison, 1990; Weiss and Fottner, 1995).

Figure 7.20 shows colour floods of streamwise vorticity coefficient, overlaid on contour lines of energy loss coefficient at two different axial locations corresponding to the measurement planes: $1.4 C_{X}$ and $2.0 C_{X}$.
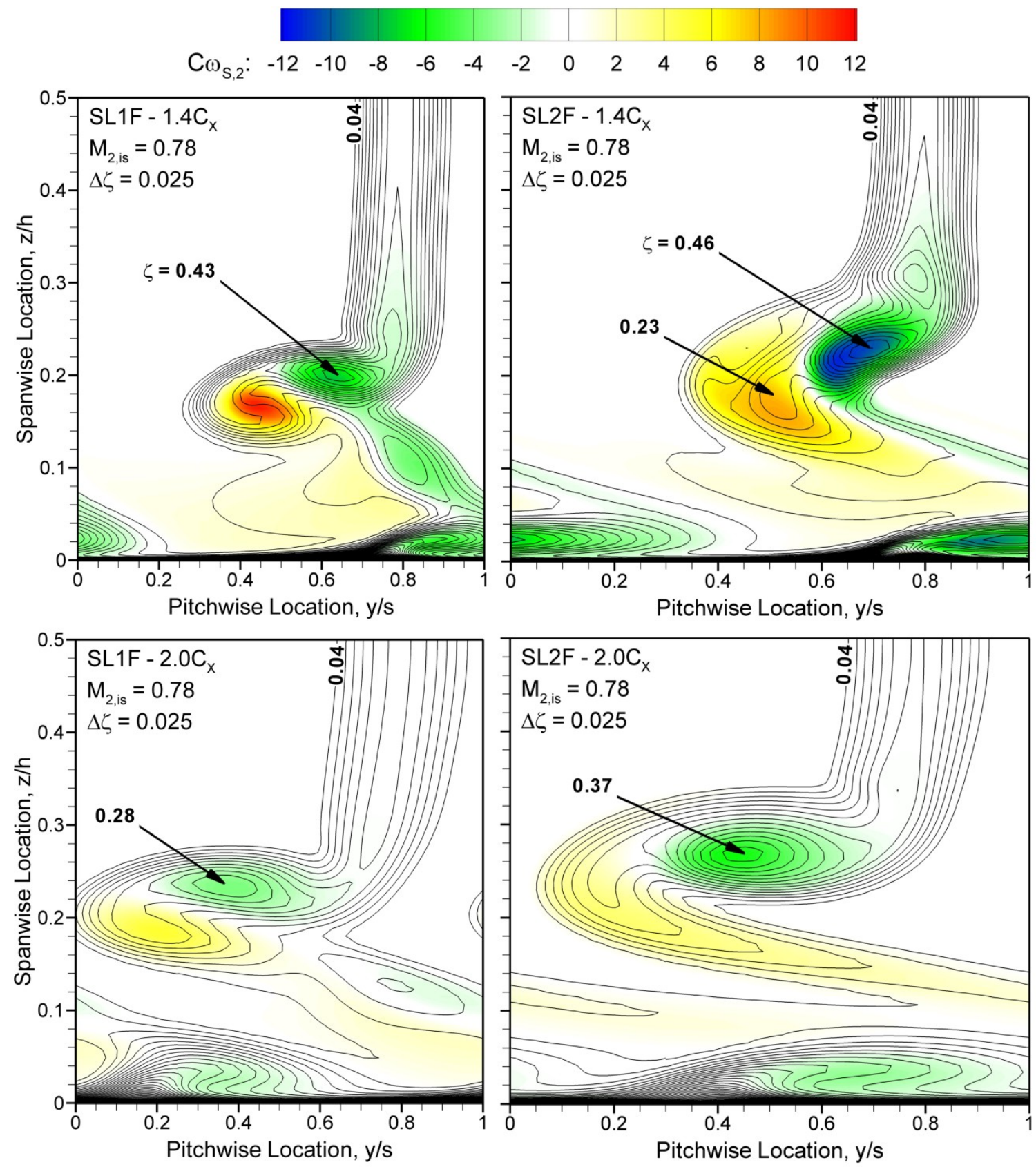

Figure 7.20 3D CFD results: colour floods of streamwise vorticity coefficient superimposed over contour lines of energy loss coefficient $\left(M_{2, i s}=0.78\right)$ 
The predicted results at $1.4 C_{X}$ in Figure 7.20 may be compared with the experimental results at the design Mach number in Figure 7.15 (please note different $C \omega_{S}$ scales). The experimental results display larger lossy fluid regions and smaller vorticity coefficients than the CFD predictions. The predicted energy loss coefficients are in good trend-wise agreement with the experiments at $1.4 C_{X}$ : SL1F displays lower secondary flow penetration depth than SL2F and smaller secondary loss peaks $(\zeta=0.43$ for SL1F versus 0.46 for SL2F). The loss peaks in the experiments in Figure 7.15, however, are significantly smaller $(\zeta=0.23$ for SL1F versus 0.26 for SL2F). In Figure 7.20, SL2F produces larger losses near the endwall than SL1F as a result of a stronger corner vortex. In each case, the dominant loss core coincides with the position of the counter vortex.

The predicted profile and secondary losses are larger than the measure values in both cases. This is in part attributed to the higher dissipation rates and the slower diffusion rates in the computations, which are largely attributable to the turbulence model. Overall, it seems that the CFD underpredicts the interaction between the low-momentum fluid (lossy fluid) and the inviscid flow, resulting in rather confined loss cores at $1.4 C_{X}$. Secondary losses increase downstream of $1.4 C_{X}$ due to both primary and secondary kinetic energy dissipation, and the near-wall losses become more prominent at $2.0 C_{X}$. The losses at $2.0 C_{X}$ are also overpredicted by the CFD, in comparison to the experimental results in Figure 7.18(a).

The measured and computed streamwise vorticity coefficients in Figure 7.15 and Figure 7.20 show marked differences at $1.4 C_{X}$ : CFD predicts higher peak vorticities, in particular associated with the counter vortex for SL2F and the passage vortex for SL1F. In addition, the predicted vortical structures are located closer to midspan than in the experiments. In Figure 7.20, the counter vortex is more intense than both passage and corner vortices for SL2F, resulting in significant distortion of the lossy fluid downstream of the cascade ( $18 \%$ to $26 \%$ span at $\left.1.4 C_{X}\right)$. The counter vortex is also well defined at $2.0 C_{X}$ for SL2F as it coincides with the loss core at $27 \%$ span, whereas the passage vortex dissipates and diffuses across the pitch at around 20\% span. In contrast, the passage vortex is the dominant secondary flow feature at $1.4 C_{X}$ for SL1F in Figure 7.20, with significantly higher peak vorticity than in the experiments (Figure 7.15). An additional region of negative vorticity is predicted for SL1F from $6 \%$ to $13 \%$ span, which coincides 
with a relatively weak loss core. In Figure 7.20, streamwise vorticity drops significantly between $1.4 C_{X}$ and $2.0 C_{X}$, and this is accompanied with additional losses and the migration of the secondary flows towards the midspan.

The pitchwise mass-averaged results from the experiments and the CFD are presented in Figure 7.21 and Figure 7.22. Within the profile flow regions, the predicted exit flow angles are in good agreement with the measurements for both SL1F and SL2F. The numerical results, however, display larger secondary flow penetration depths and larger flow underturning induced by the passage vortex in both cases (by about $2^{\circ}$ ). Flow overturning is captured quite accurately by the CFD below $10 \%$ span. Near the endwall, flow overturning is reduced by the action of the counter-rotating corner vortices, which is especially evident for SL2F at $1.4 C_{X}$ in Figure 7.22.

The predicted $C_{S K E}^{\prime}$ and energy loss coefficients are significantly higher than the measured values; however, the overall trends with downstream distance are similar. In particular, CFD overpredicts the secondary loss generation due to the more intense vortical structures in Figure 7.20.
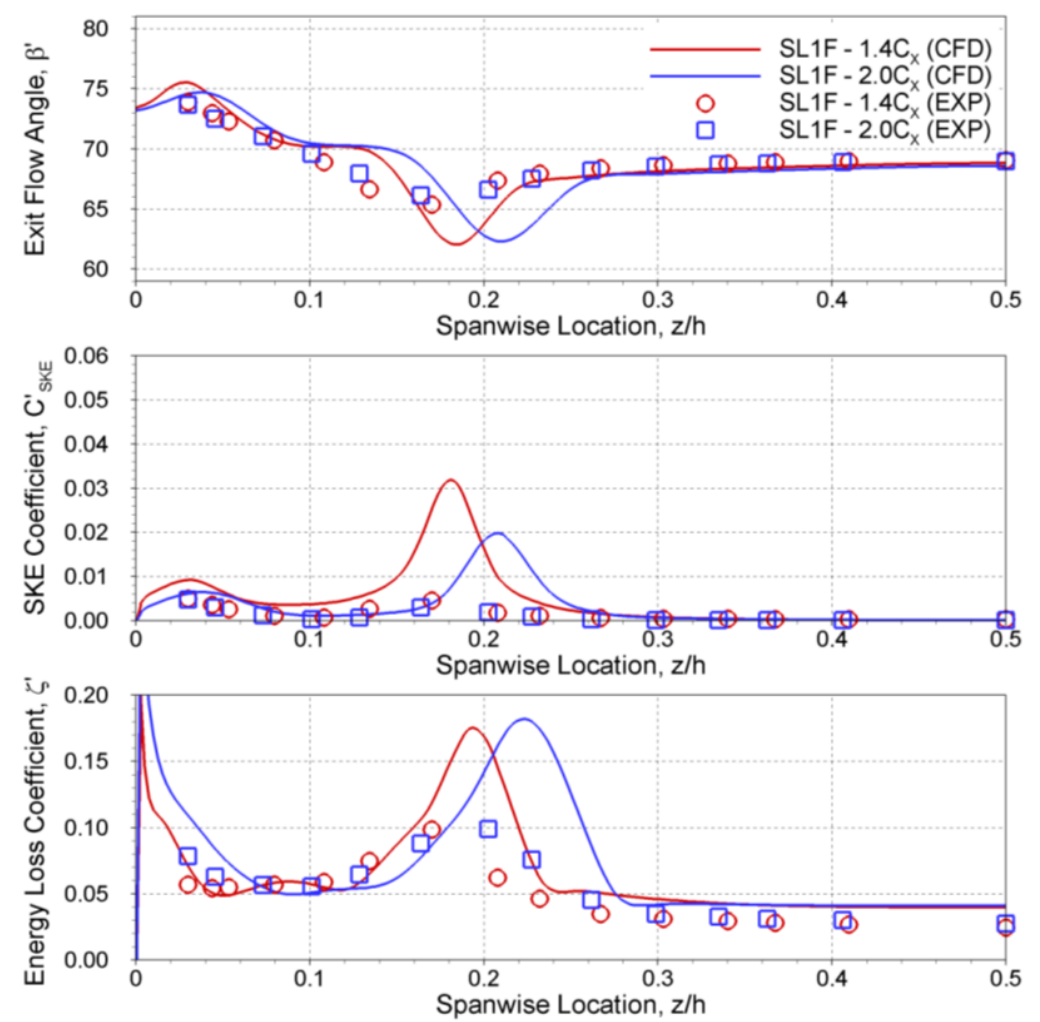

Figure 7.21 Experimental and numerical pitch-averaged results for SL1F at 1.4C $C_{X}$ : exit flow angles, $S K E$ coefficients and energy loss coefficients $\left(M_{2, i s}=0.78\right)$ 

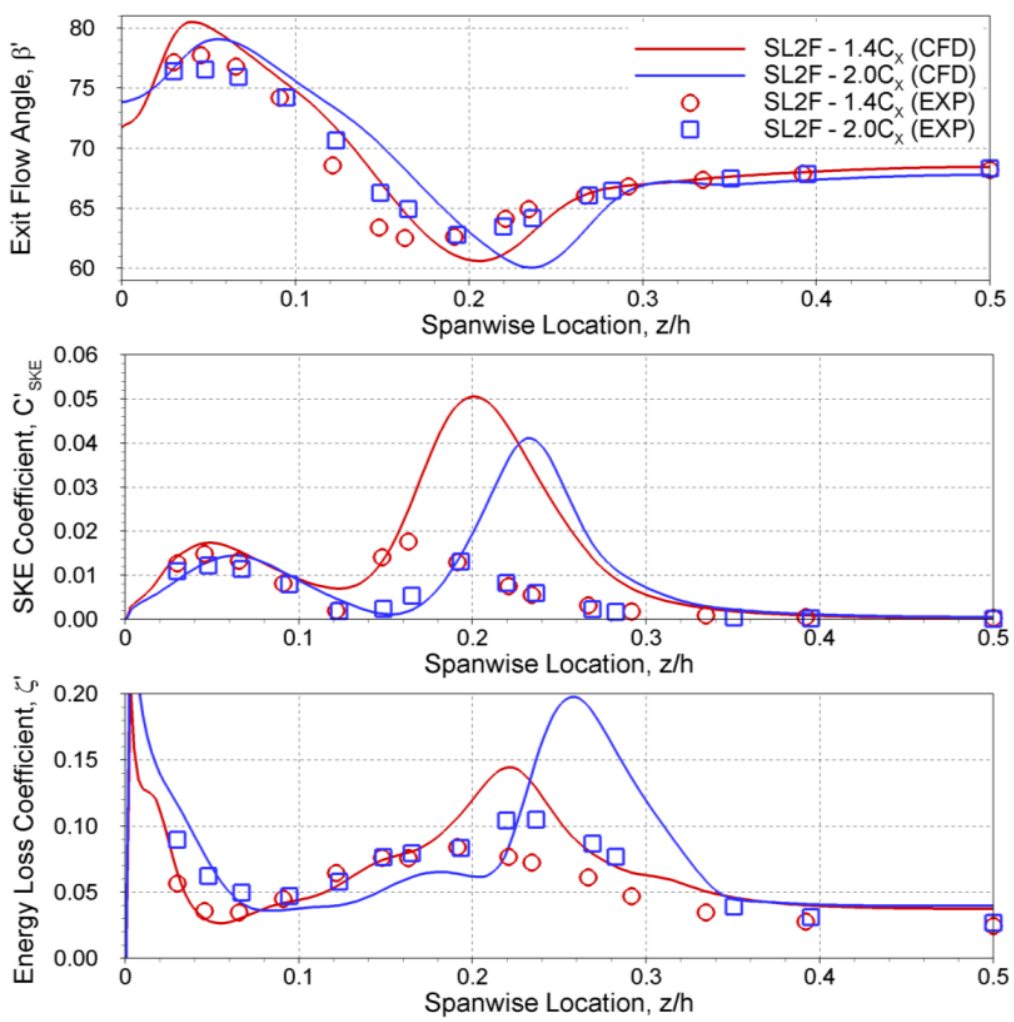

Figure 7.22 Experimental and numerical pitch-averaged results for SL2F at 1.4C $C_{X}$ : exit flow angles, $S K E$ coefficients and energy loss coefficients $\left(M_{2, i s}=0.78\right)$

As previously discussed, the 3D CFD results presented here are from fully-turbulent simulations. It should, however, be noted that MacIsaac and Sjolander (2011) also described differences between transitional CFD and measured results in a low-speed cascade; these differences were in part attributed to the application of the SST turbulence model, with isotropic eddy viscosity, to the highly-anisotropic secondary flow field. Due to current limitations in terms of computational resources, it was not possible to consider alternative CFD methods such as time-averaged unsteady RANS (URANS) or large eddy simulation (LES).

\subsection{Overall Integrated Results: Mass Averaged and Mixed Out}

The total pressure losses and the secondary kinetic energy coefficients are integrated over the entire measurement plane to quantify the performance of the blade rows. The results are both mass averaged at the traverse plane and also mixed out to uniform outlet flow conditions. To evaluate the row loss coefficients, the inlet endwall boundary layer losses (measurements at $-2.0 C_{X}$ ) are subtracted from the total pressure deficit at the 
downstream traverse plane $\left(1.4 C_{X}\right.$ or $\left.2.0 C_{X}\right)$. The profile and secondary losses are evaluated using two different loss-breakdown schemes. The energy loss coefficients, $\zeta_{P}$ and $\zeta_{S}$, are determined based on the classical loss-breakdown method, which assumes constant profile losses across the span at the midspan value. The results are presented in Table 7.5 and Table 7.6. The total pressure loss coefficients, $Y_{P}$ and $Y_{S}$, are calculated using both the classical method, and the method of Benner et al. (2006a) based on the penetration depth of the passage vortex separation line at the trailing edge $\left(Z_{T E}\right)$. The results are presented in Table 7.7 and Table 7.8. Additional details regarding the loss coefficient and the loss breakdown schemes are provided in Chapter 4.

Table 7.5 shows the integrated results at the design Mach number for all but one case: no measurements were collected at $2.0 C_{X}$ for SL4F. SL2F generates larger $C^{\prime \prime}{ }_{S K E}$ than other cascades at both measurement planes; this is attributed to the stronger secondary flow structures. The similarly-loaded SL4F cascade produces lower secondary kinetic energy than SL2F due to smaller total flow turning. The dissipation of the secondary kinetic energy downstream of $1.4 C_{X}$ is most pronounced for SL2F, although the mixing is not complete by $2.0 C_{X}$ (dissipation of the remaining $C^{\prime \prime}{ }_{S K E}$ contributes to the final mixedout losses).

Table 7.5 Overall integrated results at the design Mach number

\begin{tabular}{|c|c|c|c|c|c|c|c|c|}
\hline & \multirow{2}{*}{\multicolumn{4}{|c|}{$1.4 C_{x}$}} & \multirow{2}{*}{\multicolumn{4}{|c|}{$2.0 C_{x}$}} \\
\hline & & & & & & & & \\
\hline & SL1F & SL2F & SL3F & SL4F & SL1F & SL2F & SL3F & SL4F \\
\hline Zweifel Coefficient, Zw & 0.75 & 0.97 & 0.78 & 1.01 & 0.75 & 0.97 & 0.78 & 1.01 \\
\hline Total Flow Turning, $\theta\left(^{\circ}\right)$ & 112 & 112 & 90 & 90 & 112 & 112 & 90 & 90 \\
\hline \multicolumn{9}{|l|}{ Mass-Averaged } \\
\hline SKE Coefficient, C" SKE & 0.0014 & 0.0056 & 0.0008 & 0.0017 & 0.0010 & 0.0042 & 0.0008 & \multirow{4}{*}{$\mathrm{n} / \mathrm{a}$} \\
\hline Energy Loss Coefficient, $\zeta$ & 0.035 & 0.036 & 0.031 & 0.033 & 0.042 & 0.048 & 0.036 & \\
\hline Profile Energy Loss Coefficient, $\zeta_{P}$ & 0.024 & 0.024 & 0.020 & 0.022 & 0.027 & 0.026 & 0.021 & \\
\hline Secondary Energy Loss Coefficient, $\zeta \mathrm{s}$ & 0.011 & 0.012 & 0.011 & 0.011 & 0.015 & 0.022 & 0.015 & \\
\hline \multicolumn{9}{|l|}{ Mixed-Out } \\
\hline Energy Loss Coefficient, $\zeta$ & 0.040 & 0.045 & 0.035 & 0.038 & 0.045 & 0.056 & 0.040 & \multirow{3}{*}{$\mathrm{n} / \mathrm{a}$} \\
\hline Profile Energy Loss Coefficient, $\zeta_{P}$ & 0.025 & 0.025 & 0.020 & 0.023 & 0.027 & 0.026 & 0.021 & \\
\hline Secondary Energy Loss Coefficient, $\zeta_{\mathrm{s}}$ & 0.015 & 0.020 & 0.015 & 0.015 & 0.018 & 0.030 & 0.019 & \\
\hline
\end{tabular}


The high-turning cascades produce larger profile losses than the low-turning airfoils, and this is in part attributed to the separation zones on the suction surfaces (both at the suction peak and at the trailing edge in Figure 7.12). At 2.0 $C_{X}$, SL2F displays larger mass-averaged secondary losses than both SL1F and SL3F. The additional losses generated between $1.4 C_{X}$ and $2.0 C_{X}$ are associated with primary and secondary kinetic energy dissipation, as well as changes in static pressure. $C^{\prime \prime}{ }_{S K E}$ dissipation accounts for about $15 \%$ of the additional secondary losses generated between the measurements planes in SL2F.

The mixed-out losses are larger than the mass-averaged losses since they account for the additional energy dissipation downstream of the traverse planes. The mixed-out results from $1.4 C_{X}$, however, do not include the near-wall losses between $1.4 C_{X}$ and $2.0 C_{X}$. The near-wall losses, which are increased by the interaction between the passage and corner vortices and the endwall boundary layers, also contribute to the secondary losses. The mixed-out results from $2.0 C_{X}$ indicate significantly larger secondary losses for SL2F $\left(\zeta_{S}=0.30\right)$ than the other cascades in the present study.

Table 7.6 shows the integrated results at off-design Mach numbers for the highturning cascades. Raising the exit Mach number results in smaller Zweifel coefficients, smaller secondary kinetic energy coefficients and lower mass-averaged row losses in both cascades. Accordingly, the mixed-losses also decrease with Mach number. The reduction in secondary losses with Mach number will be revisited in Chapter 8 with reference to the endwall contouring results.

The mixed-out total pressure loss coefficients are presented in Table 7.7 and Table 7.8. In Table 7.7, the baseline-loaded cascades (SL1F and SL3F) show lower secondary flow penetration depths than the more highly-loaded cascades, although the differences are small. Based on the new loss breakdown scheme, SL2F generates higher secondary losses than profile losses (mixed-out from $1.4 C_{X}$ ). The loss trends in Table 7.7 are in agreement with the mixed-out energy loss coefficients in Table 7.5, in the sense that SL2F produces higher secondary losses and higher row losses than the other cascades at design conditions. The potential for weight reduction associated with the increased blade spacing in SL2F seems to have been offset by the additional losses, whereas SL4F might be explored further as a suitable alternative. 
Table 7.6 Overall integrated results at off-design Mach numbers (high-turning cascades)

\begin{tabular}{|c|c|c|c|c|c|c|}
\hline \multirow{3}{*}{ Outlet Isentropic Mach Number, $M_{2, \text { is }}$} & \multirow{2}{*}{\multicolumn{3}{|c|}{ SL1F }} & \multirow{2}{*}{\multicolumn{3}{|c|}{ SL2F }} \\
\hline & & & & & & \\
\hline & 0.65 & 0.78 & 0.91 & 0.65 & 0.78 & 0.91 \\
\hline Zweifel Coefficient, Zw & 0.81 & 0.75 & 0.66 & 1.07 & 0.97 & 0.89 \\
\hline \multicolumn{7}{|l|}{ Mass-Averaged } \\
\hline SKE Coefficient, C" SKE & 0.0016 & 0.0014 & 0.0014 & 0.0065 & 0.0056 & 0.0050 \\
\hline Energy Loss Coefficient, $\zeta$ & 0.037 & 0.035 & 0.032 & 0.038 & 0.036 & 0.033 \\
\hline Profile Energy Loss Coefficient, $\zeta_{P}$ & 0.025 & 0.024 & 0.023 & 0.024 & 0.024 & 0.023 \\
\hline Secondary Energy Loss Coefficient, $\zeta_{s}$ & 0.012 & 0.011 & 0.009 & 0.014 & 0.012 & 0.010 \\
\hline \multicolumn{7}{|l|}{ Mixed-Out } \\
\hline Energy Loss Coefficient, $\zeta$ & 0.042 & 0.040 & 0.038 & 0.048 & 0.045 & 0.041 \\
\hline Profile Energy Loss Coefficient, $\zeta_{P}$ & 0.026 & 0.025 & 0.024 & 0.026 & 0.025 & 0.025 \\
\hline Secondary Energy Loss Coefficient, $\zeta_{s}$ & 0.016 & 0.015 & 0.014 & 0.022 & 0.020 & 0.016 \\
\hline
\end{tabular}

Table 7.7 Mixed-out total pressure loss coefficients based on the method of Benner et al. (2006a) at the design Mach number

\begin{tabular}{|c|c|c|c|c|}
\hline Mixed-Out Results from $1.4 \boldsymbol{C}_{\boldsymbol{X}}$ & SL1F & SL2F & SL3F & SL4F \\
\hline Penetration Depth, $\boldsymbol{Z}_{\boldsymbol{T}} / \boldsymbol{h}$ & 0.09 & 0.10 & 0.08 & 0.10 \\
\hline Total Pressure Loss Coefficient, $\boldsymbol{Y}$ & 0.050 & 0.056 & 0.043 & 0.045 \\
\hline Midspan Loss Coefficient, $\boldsymbol{Y}_{\boldsymbol{M} \boldsymbol{S}}$ & 0.030 & 0.030 & 0.024 & 0.027 \\
\hline Profile Loss Coefficient, $\boldsymbol{Y}_{\boldsymbol{P}}$ & 0.027 & 0.027 & 0.022 & 0.024 \\
\hline Secondary Loss Coefficient, $\boldsymbol{Y}_{\boldsymbol{S}}$ & 0.023 & 0.029 & 0.021 & 0.021 \\
\hline
\end{tabular}

Table 7.8 Mixed-out total pressure loss coefficients based on the classical loss-breakdown method at the design Mach number

\begin{tabular}{|c|c|c|c|c|}
\hline Mixed-Out Results from $\mathbf{1 . 4}_{\boldsymbol{X}}$ & SL1F & SL2F & SL3F & SL4F \\
\hline Total Pressure Loss Coefficient, $\boldsymbol{Y}$ & 0.050 & 0.056 & 0.043 & 0.045 \\
\hline Profile Loss Coefficient, $\boldsymbol{Y}_{\boldsymbol{P}}$ & 0.030 & 0.030 & 0.024 & 0.027 \\
\hline Secondary Loss Coefficient, $\boldsymbol{Y}_{\boldsymbol{S}}$ & 0.020 & 0.026 & 0.019 & 0.019 \\
\hline
\end{tabular}




\section{Chapter 8}

\section{Secondary Flows and Losses in Turbine Cascades with Contoured Endwalls}

\subsection{Introduction}

Secondary losses due to endwall flows constitute as much as a third of the total entropy generation within the turbine stages of an aircraft engine (Denton, 1993). Consequently, researchers have investigated several methods for reducing the secondary losses in turbines, with much focus on endwall contouring in the past decade. The majority of the investigations in the published literature have been conducted in lowspeed linear cascade facilities, which neglect the compressibility effects, and rotating test rigs with some inherent limitations on the spatial resolution of the experiments. The current study is therefore aimed to improve the understanding of the secondary loss generating mechanisms and to assess the effectiveness of endwall contouring in compressible flows, using surface flow visualization and steady-state probe measurements from transonic linear turbine cascades.

Endwall contouring is utilized to modify the near-wall pressure distribution, and thereby to control the development of the vortical structures within the blade passages. These objectives are accomplished through streamline curvature: flow is locally accelerated over convex features and decelerated over concave features. The profiled endwall may be axisymmetric or non-axisymmetric, extending beyond or restricted to within the blade passage, and combined with three-dimensional blades and leading/trailing edge fillets at the endwalls. For the present study, non-axisymmetric endwall contours were designed using the method of Praisner et al. (2007) with no blade modifications or fillets. The contoured endwalls were optimized computationally to minimize the predicted mass-averaged row losses in the transonic flow regime. 
The experimental and computational results from the high-turning turbine cascades are presented in this chapter. The baseline flat-endwall configurations are referred to as SL1F and SL2F: SL2F is more highly loaded than SL1F. The results for these cascades were presented in the last chapter. The corresponding contoured-endwall configurations are referred to as SL1C and SL2C.

\subsection{Non-Axisymmetric Endwall Contouring Design}

\subsubsection{Introduction}

Several researchers have presented design methods for non-axisymmetric endwall contouring (Harvey et al., 2000; Brennan et al., 2003; Germain et al., 2010; Sonoda et al., 2009). The computationally-optimized designs are typically validated through experimental investigations in linear cascades (Hartland et al., 2000; Ingram et al., 2005a; Gregory-Smith et al., 2008) and rotating test rigs (Harvey et al., 2002; Schuepbach et al., 2007). For the present study, the contoured endwalls were designed by researchers from Pratt and Whitney Aircraft (PWA) using a gradient-based optimization algorithm coupled with compressible CFD simulations. The design and optimization procedures have been described by Praisner et al. (2007), and their application to several low-speed linear cascades has been examined by Knezevici (2011). In general, the CFD simulations and the low-speed cascade studies have demonstrated the effectiveness of non-axisymmetric endwall contouring under incompressible flow conditions. The present study examines the application of endwall contouring to transonic turbine cascades.

\subsubsection{Computational Analysis for Design and Optimization}

An in-house (PWA) 3D structured RANS code with second order spatial accuracy was used to compute the flow fields. The simulations were performed using the $k-\omega$ turbulence model and the transition model of Praisner and Clark (2007). The computational domain, extending from the endwall to midspan, contained approximately 800,000 nodes per passage, with $\mathrm{y}^{+}$of about 1.0 for the first node away from the wall. The endwall contouring was designed using commercial optimization software with the goal of minimizing the mass-averaged row losses (objective function). The following additional constraints were imposed: 
- The contouring was to be confined to the blade passage, referred to as "fullpassage" contouring, with 25 control points ( 5 pitchwise x 5 streamwise) and one degree-of-freedom in the wall-normal direction, as shown in Figure 8.1.

- The flow capacity was conserved by matching the flat-endwall cases for midspan loading distributions, axial velocity density ratio, throat areas and midspan exit flow angles.

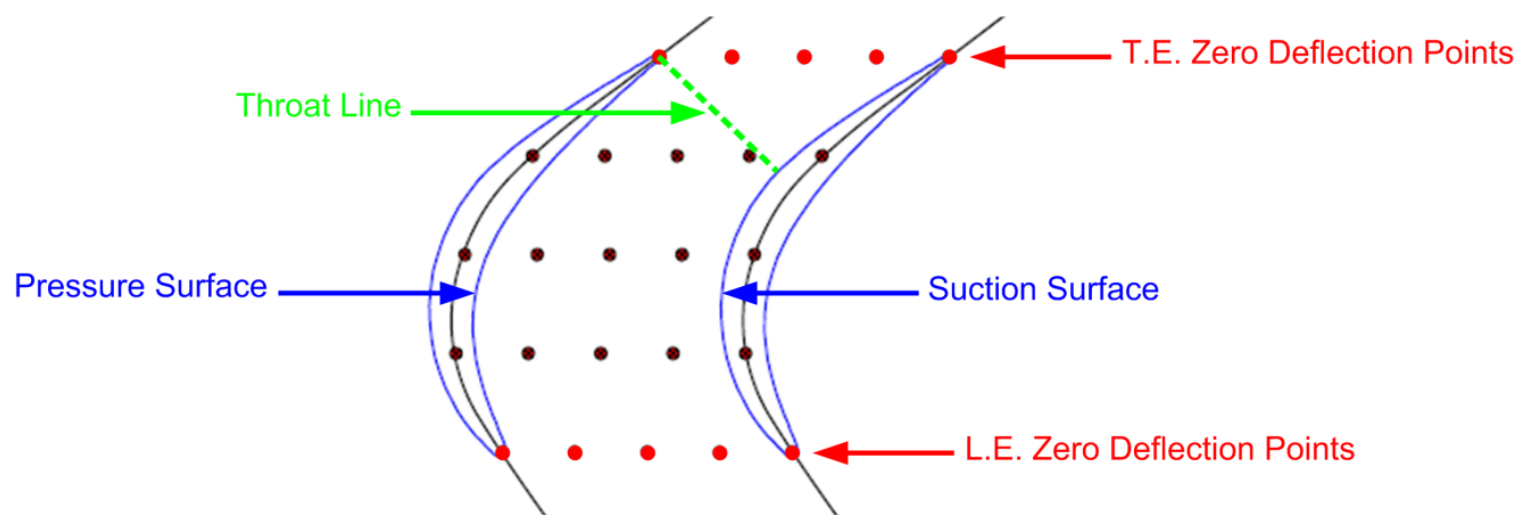

Figure 8.1 Example distribution of endwall control points (reproduced from Praisner et al., 2007)

As many as 1000 design iterations were performed to arrive at the final optimized contoured geometry, at which point other parameters such as turbulence kinetic energy $(T K E)$ and secondary kinetic energy ( $S K E)$ were examined to substantiate the improvements. As a final design criterion, solutions with increased overturning near the endwall at 5\% span were rejected to ensure acceptable spanwise exit flow uniformity.

Additional simulations were performed to investigate the effects of the transition model, inlet free-stream turbulence and inlet endwall boundary layer thickness on the predicted row losses. The simulations with the transition model generate approximately $10 \%$ lower losses for both flat and contoured cascades, compared to the fully turbulent simulations. Nevertheless, the computational results indicate very similar benefits from contouring in all cases.

The PWA CFD predictions indicate mass-averaged row loss reductions of 3\% for SL1C and 7\% for SL2C, relative to the corresponding flat-endwall cases. The contoured cascades also have weaker vortical structures and produce lower SKE. These results are in agreement with the low-speed findings of Praisner et al. (2007). 


\subsubsection{Geometric Features of the Endwalls}

The optimized endwall geometries are shown in Figure 8.2 and Figure 8.3. The contoured endwalls include two distinct protrusions "P" and one primary depression "D" (in the pressure side of the passage). SL2C displays a second depression " $\mathrm{D}_{2}$ " in the aft part of the passage along the pressure surface. A cross-passage ridge " $R$ " is also identified for SL2C, whereas the ridge is located closer and almost parallel to the pressure surface for SL1C. The difference between the two configurations is the result of the optimization software arriving at two different solutions (unknown a priori). As shown in Table 8.1, the endwall spanwise displacement due to the contouring varies from about $-6 \%$ to $+19 \%$ of the axial chord length $\left(C_{X}=25.4 \mathrm{~mm}\right)$. As a potential adverse effect, the application of contouring increases the endwall surface areas compared to the flat-endwall cascades: $16 \%$ increase for SL1C and 10\% increase for SL2C.
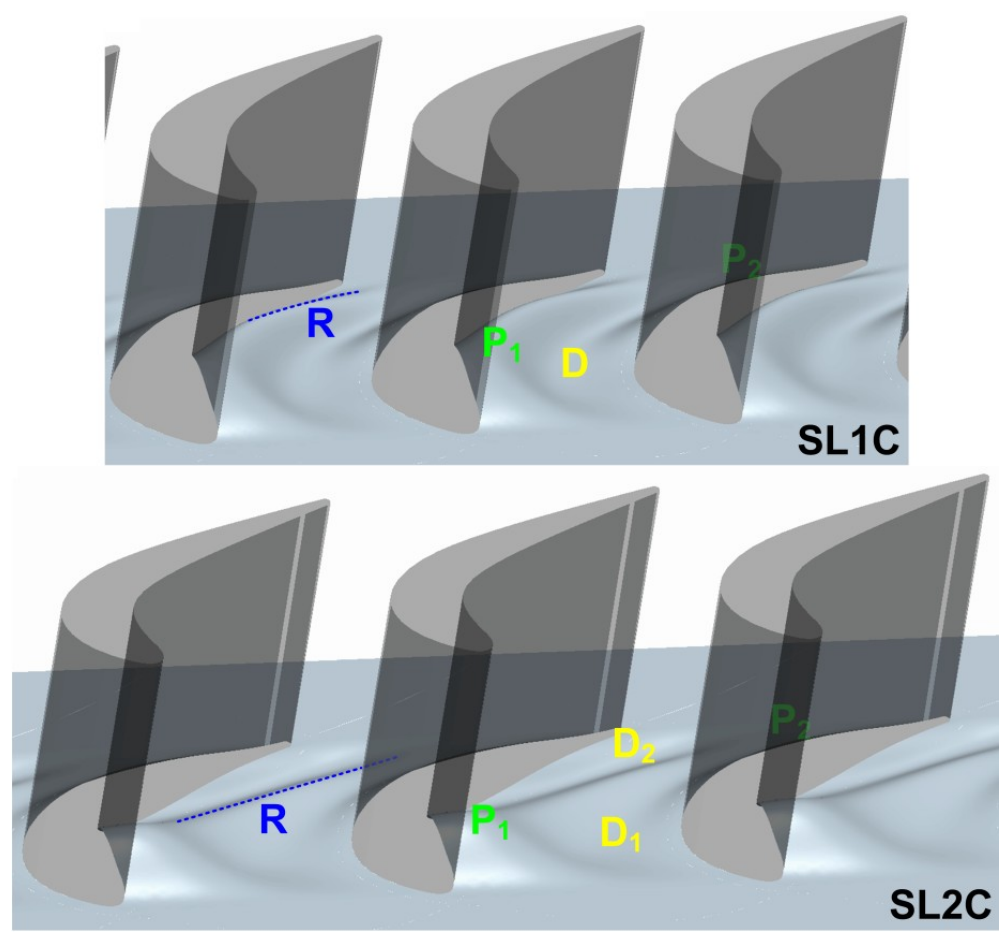

Figure 8.2 Optimized contoured endwall geometries

Table 8.1 Endwall spanwise displacement due to contouring

\begin{tabular}{|c|c|c|c|c|c|c|c|c|}
\hline \multicolumn{6}{|c|}{ Maximum Elevation Differences as Percentage of Axial Chord $\left(\% C_{X}\right)$} \\
\hline & $\mathrm{P}_{1}$ & $\mathrm{P}_{2}$ & $\mathrm{D}$ & & $\mathrm{P}_{1}$ & $\mathrm{P}_{2}$ & $\mathrm{D}_{1}$ & $\mathrm{D}_{2}$ \\
\hline SL1C & 19 & 8 & -5 & SL2C & 17 & 8 & -6 & -4 \\
\hline
\end{tabular}




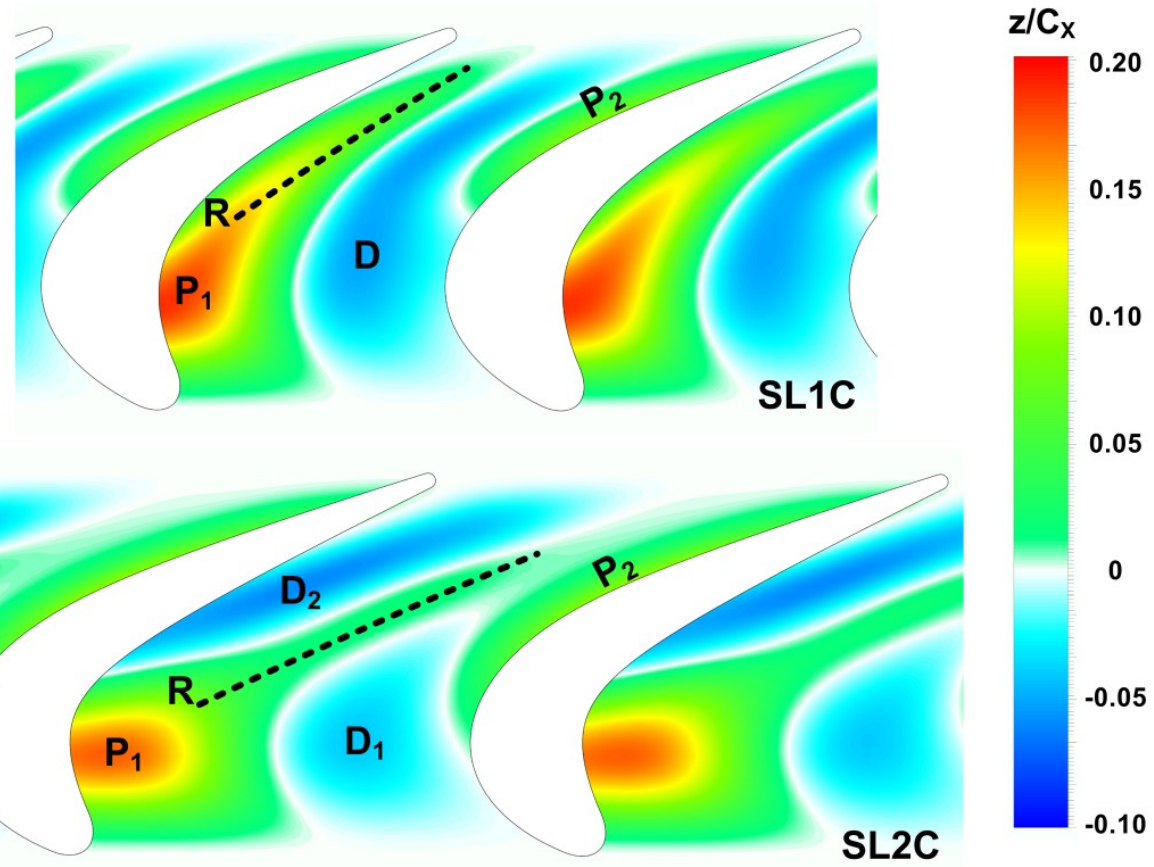

Figure 8.3 Endwall height contours $\left(z / C_{x}\right)$

Convex endwall curvature acts to locally accelerate the flow, and thereby reduce the static pressure near the pressure surface protrusion $\left(\mathrm{P}_{1}\right)$. Concave endwall curvature, on the other hand, raises the local static pressure towards the suction side of the passage depression $\left(\mathrm{D}_{1}\right)$. The modification of the endwall static pressure distribution leads to the reduction of the cross-passage pressure gradient in the forward half of the passage. This pressure gradient is typically the main driving force affecting the roll-up of the pressureside leg of the horseshoe vortex (Benner et al., 2004; Zoric et al., 2007a; Knezevici et al., 2009). Therefore, the reduction in transverse pressure gradient is expected to retard the development of the passage vortex, and delays its interaction with the suction-surface boundary layer. Additional improvements are expected as a result of a diminished counter vortex (smaller contribution from the wall-vortex induced by the passage vortex), leading to lower losses and SKE (Knezevici et al., 2010). The cross-passage ridge in the aft part of the passage is also intended to alter the trajectory of the passage vortex. The amplitude and location of the ridge was arrived at by the optimization procedure, giving rise to differences between SL1C and SL2C configurations. By the trailing edge plane the displacement due to contouring returns to the nominal endwall height since the contouring is restricted to the passage. This results in regions of adverse and favourable 
streamwise pressure gradients across the pitch. The effects of contouring on the endwall limiting streamlines will be discussed with reference to the flow visualization pictures.

\subsubsection{Predicted Airfoil and Endwall Isentropic Mach Number Distributions}

The midspan surface Mach number distributions are presented in Figure 8.4. SL2 is both more highly loaded and more mid loaded than SL1. Accordingly, SL2 has larger cross-passage pressure differences in the forward part of the passage, and larger adverse pressure gradients on the blade surface following the suction peak. On the pressure surface, SL2 has a region of high static pressure $\left(M_{i s}<0.1\right)$ from the leading edge to about $0.5 C_{X}$. The high static pressure in this region is typically found to enhance the growth of the passage vortex, resulting in higher secondary losses as shown in Chapter 7.
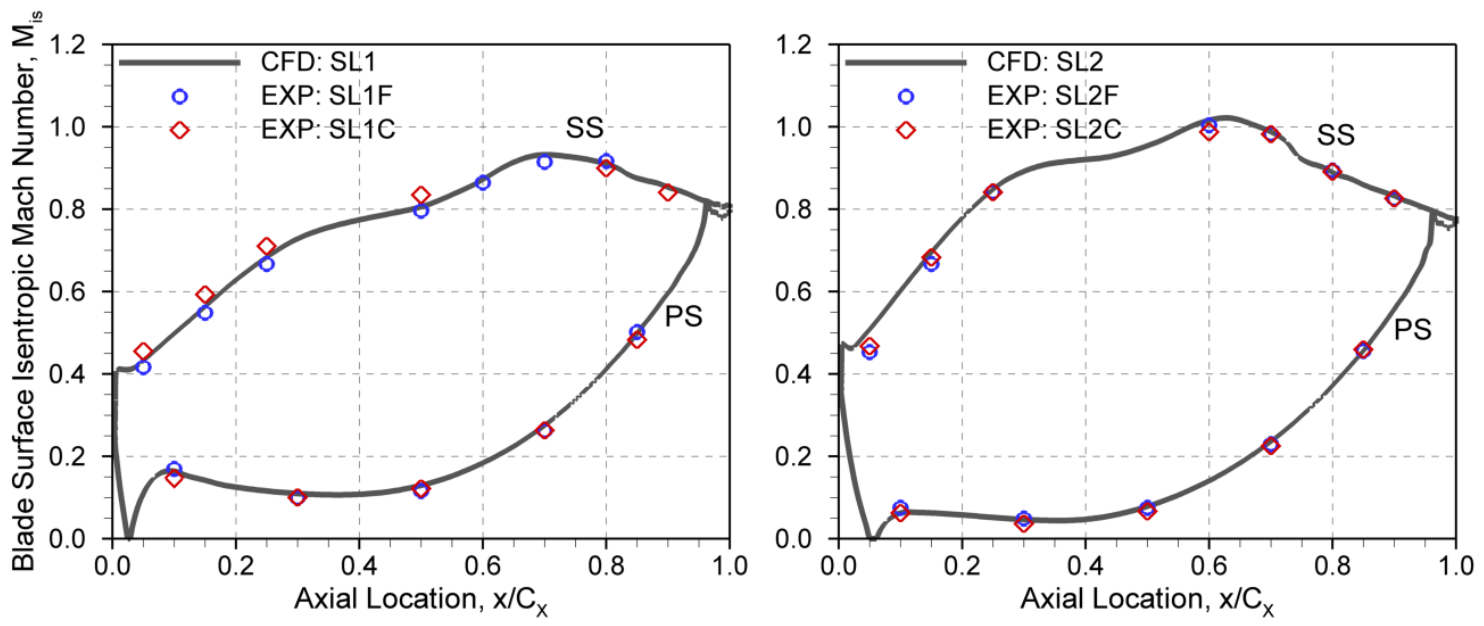

Figure 8.4 Midspan blade loading distributions $\left(M_{2, i s}=0.78\right)$

The predicted endwall isentropic Mach number distributions, based on the inlet centreline total pressure and the wall static pressures, are shown in Figure 8.5. The endwall isentropic Mach numbers are close to 1.0 near the throat region. The locations of the protrusions are also identified for the contoured cascades. One of the objectives of contouring is to increase the flow velocity in the near-stagnation regions, particularly near the pressure surface.

In Figure 8.5, the velocity vectors are extracted at $0.6 \mathrm{~mm}$ above the endwall surface, which corresponds to $1 \%$ span for the flat-endwall cases, to demonstrate the effects of 
contouring on the endwall crossflows. The velocity vectors at $0.5 \%$ and $1.5 \%$ span show very similar behaviour to the results presented here.

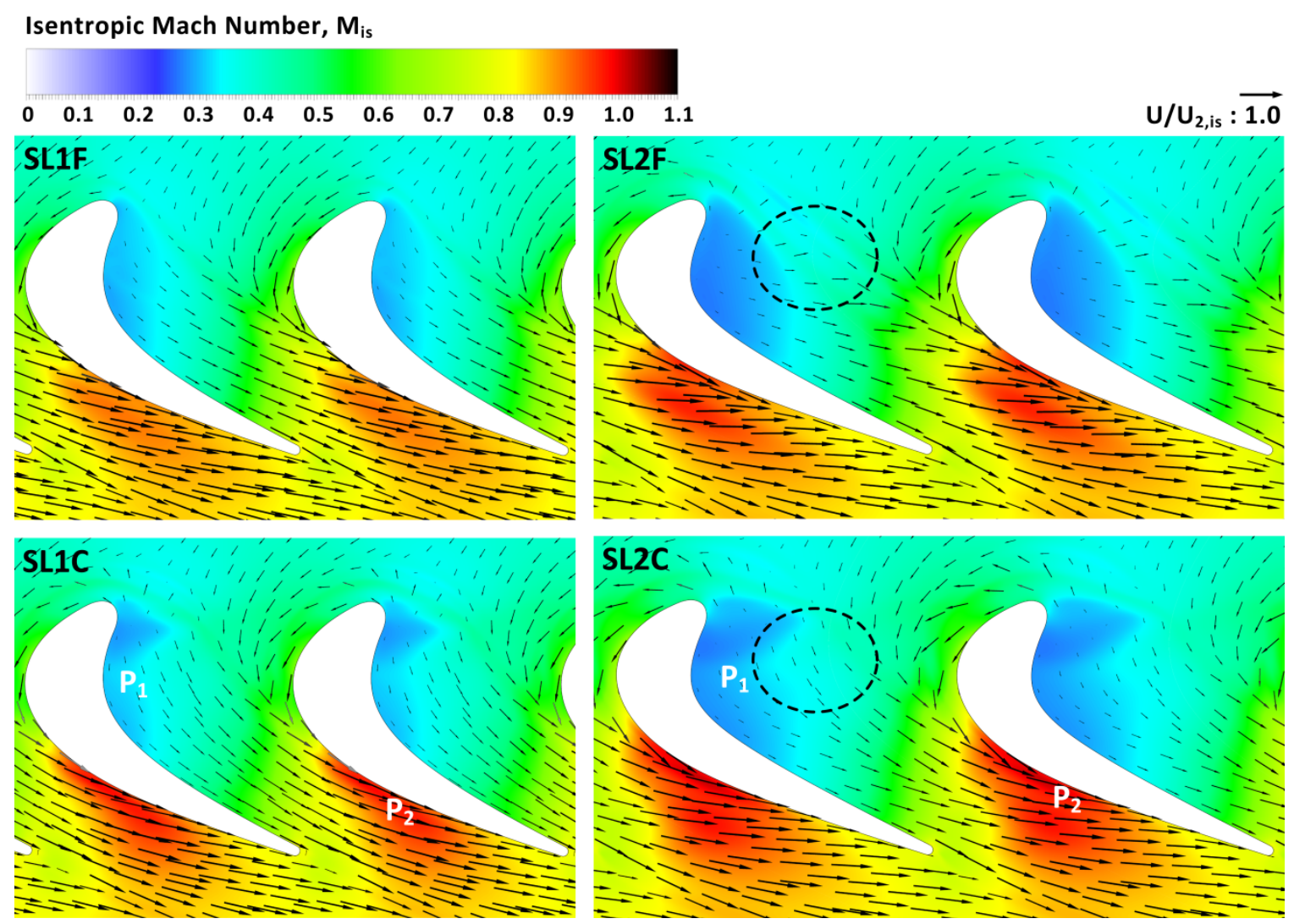

Figure 8.5 CFD predictions: endwall isentropic Mach number distributions overlaid on velocity vectors at $0.6 \mathrm{~mm}$ above the endwall surface

The protrusions locally accelerate the flow as evident by the larger endwall isentropic Mach numbers for the contoured cascades. The increase in isentropic Mach number near the pressure surface associated with $\mathrm{P}_{1}$ results in more favourably-oriented endwall crossflows. In contrast to SL2F, the near-wall velocity vectors for SL2C are aligned approximately in the streamwise direction in the forward of the passage (enclosed in dashed circle), which is confirmed by the experimental flow visualization results in the next section. The contoured cascades also display a region of flow acceleration near the suction surface in the aft part of the passage, associated with $\mathrm{P}_{2} . \mathrm{P}_{2}$ alleviates the adverse streamwise pressure gradients downstream of the suction peak, and this may weaken the formation of the corner vortex. 


\subsection{Experimental Results at the Design Mach Number}

\subsubsection{Surface Flow Visualization Results}

The surface flow visualization studies were conducted using a mixture of fluorescentdye and motor oil, illuminated and photographed under ultra-violet lighting, following the completion of the tests; the results are presented in Figure 8.6 and Figure 8.7. As in Chapter 7, the nomenclature of Sieverding (1985) is used to interpret the limiting streamlines: dashed lines are used for flow separation, dotted lines for flow reattachment and arrows for surface shear stress vectors. Also marked are the locations of the protrusions and the depressions on the contoured endwalls, while the approximate positions of the ridges are shown with cyan dashed lines. Since the surface flow visualization results for the flat-endwall cascades were discussed in Chapter 7, the focus here is on the differences due to endwall contouring.

In Figure 8.6 and Figure 8.7, $\mathrm{A}_{1}$ marks the saddle point of the inlet endwall boundary layer separation and $S_{1}$ is the resulting primary separation line. In the contoured cascades, the position of $A_{1}$ is shifted in both pitchwise and axial directions due to changes in endwall static pressure distributions. In Figure 8.6(b) and Figure 8.7(b), the inlet boundary layer streamlines approaching the blade passages experience local acceleration (over the protrusion) or diffusion (over the depression) as compared to the flat endwalls, induced by the pitchwise variations in endwall shape. The resulting streamline curvature displaces $A_{1}$ in the pitchwise direction towards the centre of the passage. $A_{1}$ and $S_{1 S}$ are also shifted upstream, indicating slightly earlier inlet boundary layer separation, which is particularly noticeable for the more highly-loaded SL2C cascade. These results, consistent with the low-speed findings of Knezevici et al. (2010) and Torre et al. (2011), also indicate changes in the horseshoe vortex formation and inlet boundary layer entrainment due to contouring.

Moving downstream, $S_{2}$ marks the lift-off line of the horseshoe vortex. In each contoured case, the distance between $\mathrm{A}_{1}$ and $\mathrm{S}_{2}$ has increased relative to the corresponding flat endwall. The main difference between SL1C and SL2C is evident in the locations of the pressure-side legs, $\mathrm{S}_{1 \mathrm{P}}$ and $\mathrm{S}_{2 \mathrm{P}}$, and this is attributed to the high static pressure regions on the endwalls stemming from the optimization procedure. For SL1C, 
the depression, $\mathrm{D}$, extends upstream of $\mathrm{S}_{2 \mathrm{P}}$ and through the blade passage at approximately $70 \%$ pitch. Consequently, the distance between $\mathrm{S}_{1 \mathrm{P}}$ and $\mathrm{S}_{2 \mathrm{P}}$ is increased if compared to Figure 8.6(a). For SL2C, on the other hand, $\mathrm{D}_{1}$ is both smaller and more localized. Furthermore, the SL2 loading distribution in Figure 8.4 indicates stronger cross-passage pressure differences in the forward part of the passage with significantly lower suction-surface static pressures than SL1, which ultimately result in smaller flow convergence between $\mathrm{S}_{1 \mathrm{P}}$ and $\mathrm{S}_{2 \mathrm{P}}$ in Figure 8.7(b). Overall, endwall contouring leads to weaker interaction between $\mathrm{S}_{2 \mathrm{P}}$ and the suction-surface boundary layers in both cases. This is typically found to reduce the size of the passage vortex and the resulting losses (Knezevici, 2009), as will be examined in the next sections.
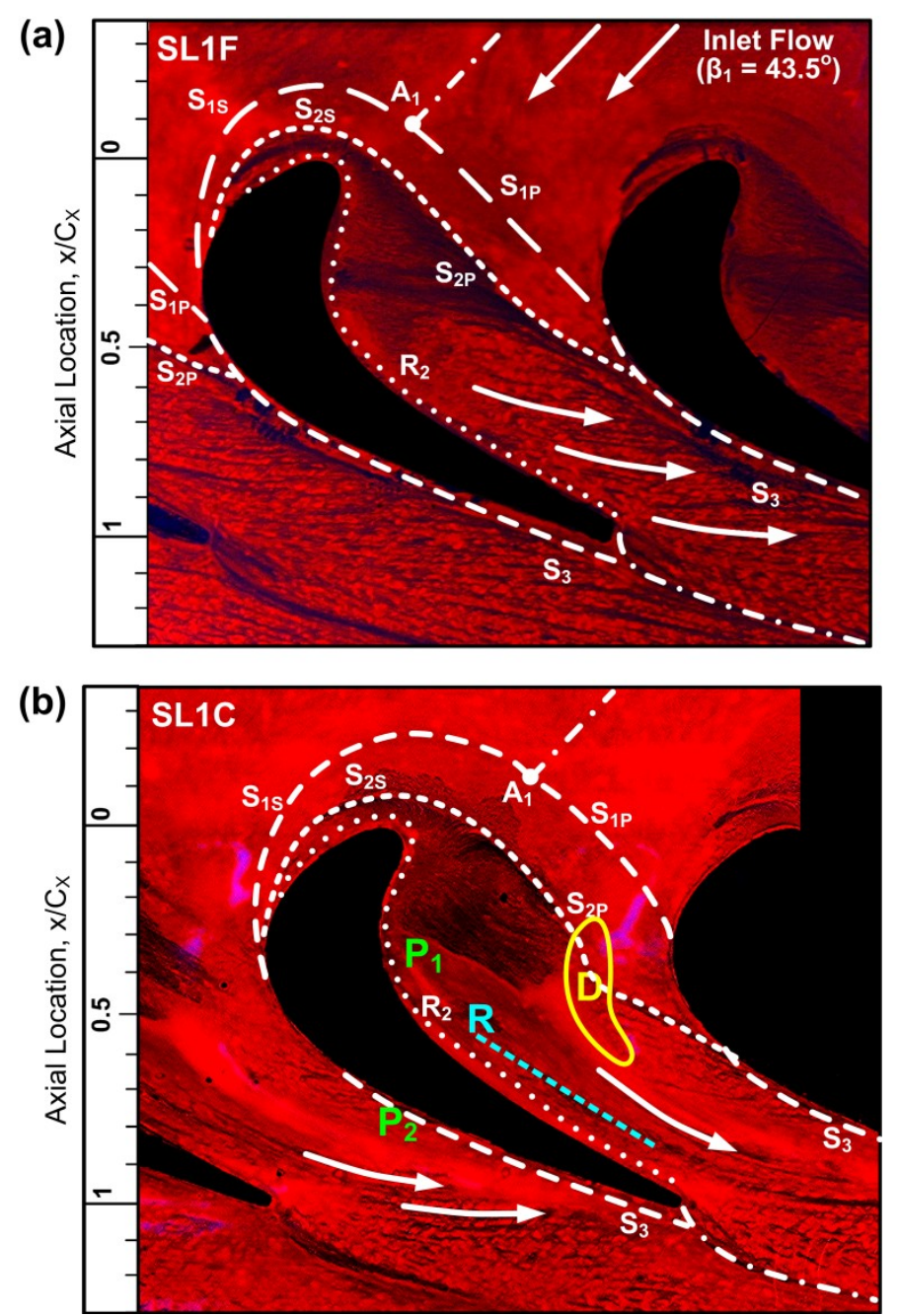

Figure 8.6 Endwall surface flow visualization results $\left(M_{2, i s} \approx 0.8\right)$ : (a) SL1F and (b) SL1C 

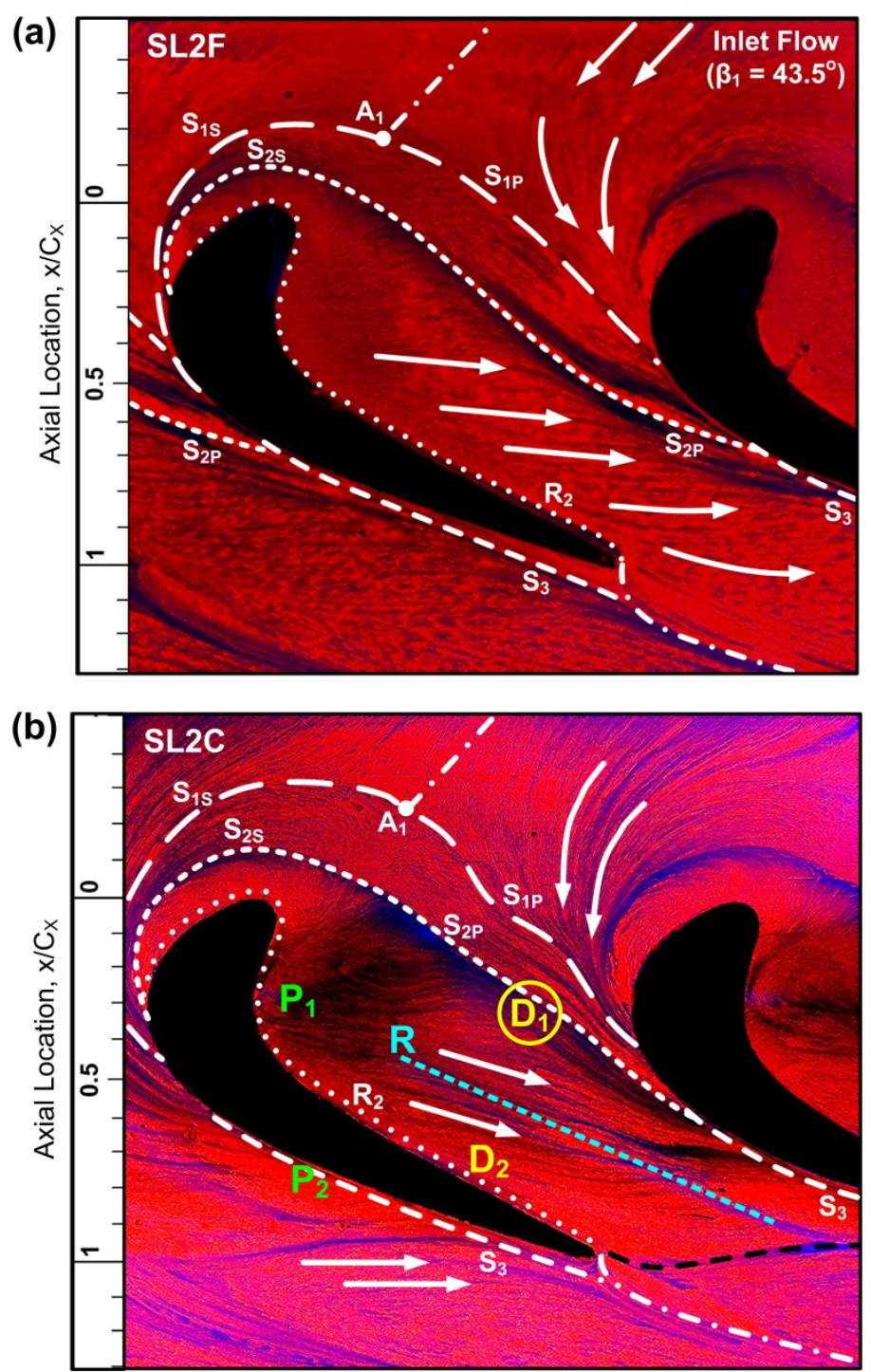

Figure 8.7 Endwall surface flow visualization results $\left(M_{2, i s} \approx 0.8\right)$ : (a) SL2F and (b) SL2C

The new endwall boundary layer, which forms after the horseshoe vortex has removed the inlet endwall boundary layer, begins in the corner at the pressure side (at $\mathrm{R}_{2}$ ). The new endwall boundary layer is swept across the passage by the transverse pressure gradients. An important objective of contouring is to reduce the strength of this crossflow within the passage. As shown in Figure 8.6(b) and Figure 8.7(b), the shear stress vectors in the contoured cascades are more closely aligned with the nominal streamwise directions up to about $0.8 C_{X}$. For SL2C, this is primarily attributed to the protrusion $\mathrm{P}_{1}$ and the streamwise ridge, which alter the trajectory of the passage vortex. Subsequently, the passage vortex likely undergoes fewer revolutions, which is anticipated to reduce the entrainment of the low-momentum endwall-boundary-layer fluid. In Figure 
8.7(b), the large surface shear stresses near $\mathrm{P}_{1}$ also suggest closer proximity of the passage vortex to the endwall. Downstream of $S_{2 P}$ and along the suction surface in Figure 8.6(b), protrusion $\mathrm{P}_{2}$ is expected to reduce the adverse streamwise pressure gradients in the aft part the passage that would otherwise enhance the passage vortex both in size and in radial migration.

SL1C and SL2C also have differences in the location and extent of the ridge and the second depression. The ridge reduces the static pressure along the pressure surface for SL1C, thereby reducing the transverse pressure difference acting on the passage vortex. For SL2C, the cross-passage ridge, originating at $\mathrm{P}_{1}$ and extending towards the suction surface, accelerates the endwall crossflow in the nominal streamwise direction. The differences due to contouring are especially evident from the mid-passage shear stress vectors in Figure 8.7(a) and Figure 8.7(b). In the aft part of the passage in Figure 8.7(b), the surface streamlines over $\mathrm{D}_{2}$ are turned towards the suction surface under the influence

of raised static pressure. This is also evident close to the trailing-edge plane, possibly leading to corner vortex intensification, as the wall displacement due to contouring returns to its initial (pre-passage) height. The results of Ingram et al. (2002) indicated a similarly stronger corner vortex. The additional losses associated with the more intense corner vortex were deemed acceptable. In Figure 8.7(b), another high shear stress line (black dashed line) is identified downstream of $\mathrm{D}_{2}$, which will be further discussed with reference to the streamwise vorticity plots. Downstream of the cascades, the corner vortices are displaced away from the suction surfaces, possibly due to trailing edge separation on the airfoil near the endwalls.

\subsubsection{Downstream Flow Field Measurements at $1.4 C_{X}$}

Figure 8.8(a) shows colour flood contour plots of streamwise vorticity coefficient, superimposed on contour lines of mass-weighted energy loss coefficient $(\zeta \times A V D R)$. As before, the results are plotted over one blade pitch $(0<y / s<1)$ and half span $(0<z / h<$ $0.5)$. The pitchwise mass-averaged flow angles, showing the classic under/overturning, are given in Figure 8.8(b). 
(a)

(a)
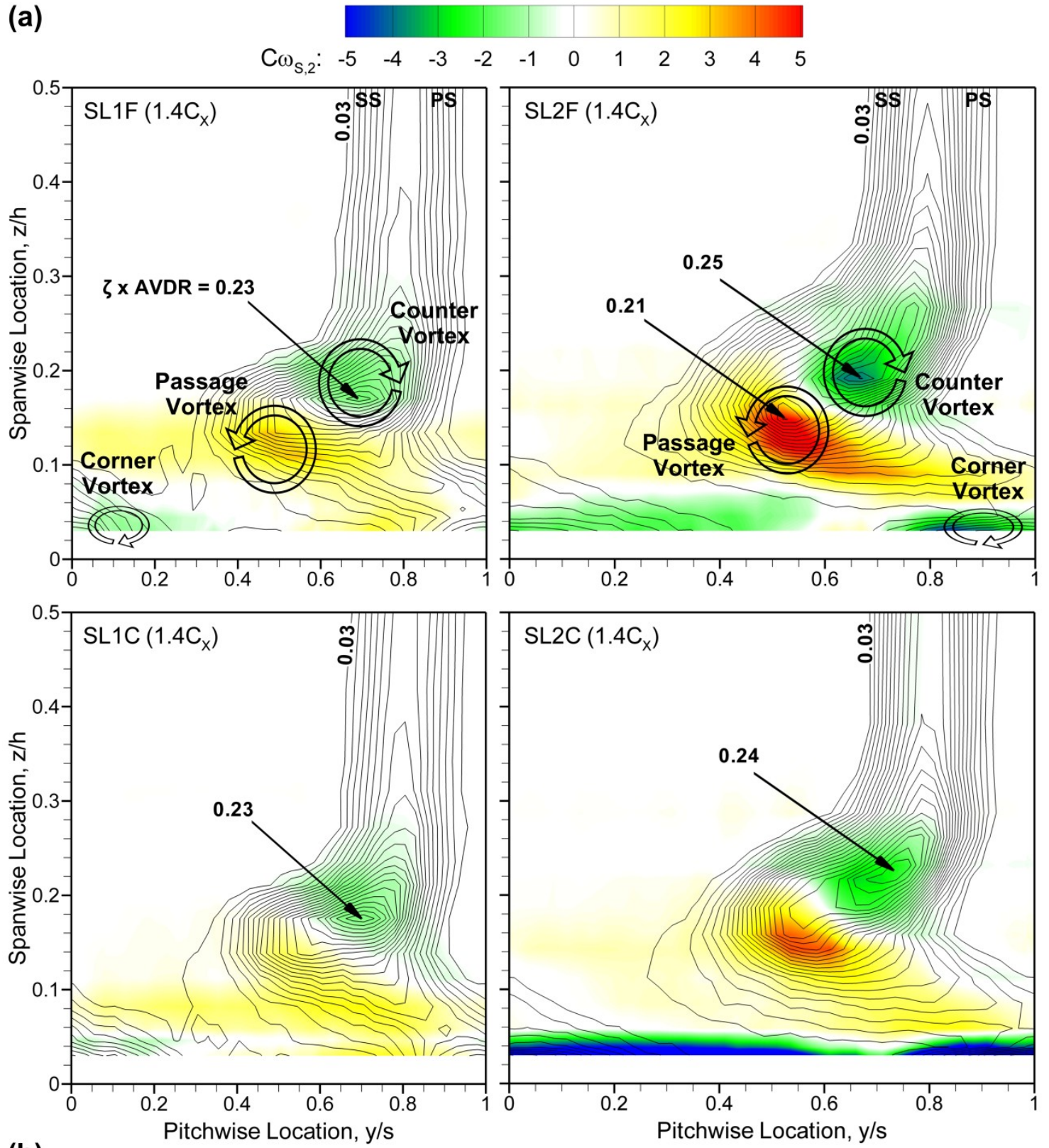

(b)

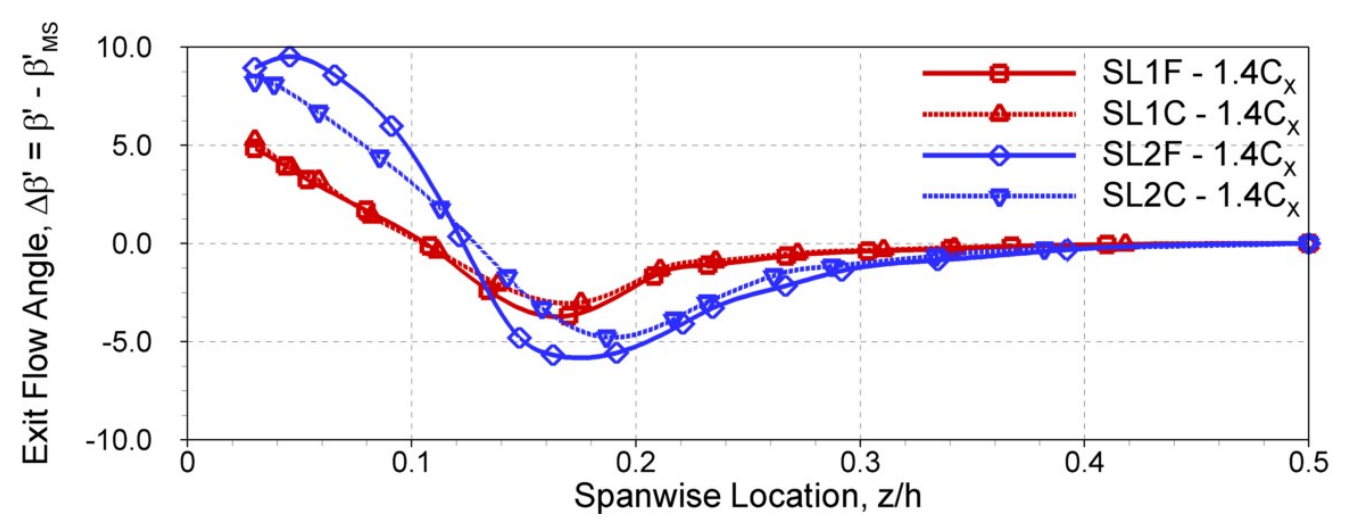

Figure 8.8 (a) Colour floods of streamwise vorticity coefficient overlaid on contour lines of mass-weighted energy loss coefficient and (b) pitch-averaged exit flow angles at $1.4 C_{X}$ 
SL1F and SL1C display similar secondary flow structures and secondary losses: the sizes and locations of the vortical structures, and the secondary loss penetration depths are not significantly altered by the contouring. The passage vortex and the counter vortex, however, display slightly lower intensities for SL1C. Consequently, the pitchaveraged exit flow angles show about a $1^{\circ}$ reduction in underturning, while there is no measureable increase in overturning near the endwall.

In general, the more highly loaded SL2 cascades display stronger secondary flow structures than the SL1 cascades. The application of endwall contouring reduces the size and strength of the passage and counter vortices for SL2C. Furthermore, SL2C displays only one distinct loss core, as opposed to two loss cores for SL2F, with a slightly lower magnitude $(\zeta \times A V D R=0.24)$. The location of the vortical structures and the pitchwise extent of the secondary losses, on the other hand, are not significantly affected by contouring. The loss core associated with the counter vortex has migrated slightly closer to midspan for SL2C, perhaps indicative of larger spanwise static pressure gradients. The intensity of the corner vortex, which stretches almost entirely across the pitch for SL2C, has increased markedly. This intensification is mainly attributed to the depression $\mathrm{D}_{2}$ in Figure 8.3, as discussed with reference to the trailing-edge shear stress line in Figure $8.7(\mathrm{~b})$.

As shown in Figure 8.8(b), contouring yields approximately $2^{\circ}$ reductions in both underturning with a small shift towards midspan and overturning below $10 \%$ span. The more uniform exit flow angle distribution, sometimes used as the objective function for contouring design (Harvey et al., 2000), would reduce the losses due to off-design incidence in any subsequent blade rows. The reduced under/overturning also affects the spanwise variations in mass flux, and consequently the mass-weighted loss contours in Figure 8.8(a): regions of underturning and overturning are associated with high and low mass fluxes, respectively. Accordingly, SL2C displays higher mass-weighted loss coefficients than SL2F from 5\% to $10 \%$ span. In summary, the application of contouring results in weaker secondary flow structures, but it does not reduce the secondary losses at $1.4 C_{X}$. These results do not confirm the mass-averaged row loss reductions, predicted by PWA CFD. This suggests that the simulations overpredict the loss generation due to mixing within the blade passages. 
Figure 8.9(a) shows colour floods of secondary kinetic energy coefficient $\left(C_{S K E}\right)$, overlaid on secondary velocity vectors. Also shown in Figure 8.9(b) are the pitchwise mass-averaged distributions of $C_{S K E}$. $C_{S K E}$ is mainly a function of the secondary velocities, and to a lesser extent of the local variations in density. Large values of $C_{S K E}$ are found in the interaction regions between the passage vortex and the counter vortex. Closer to the endwall, large pitchwise secondary velocities are induced by the overturning action of the passage vortex.

The application of contouring results in slightly smaller secondary velocities and $C_{S K E}$ for SL1C. This is particularly evident for the passage and counter vortices, as anticipated from Figure 8.8(a). The smaller $S K E$ at $1.4 C_{X}$ will also reduce the mixing losses downstream, which will be discussed in the next sections. Consistent with the findings in Figure 8.8(a), the contouring features for SL1C (the baseline-loaded case) do not bring about any deleterious effects. The SL2C results in Figure 8.9(a) show significant improvements compared to SL2F: up to $30 \%$ reduction in the magnitude of the secondary velocities and $50 \%$ reduction in peak values of $C_{S K E}$ between $10 \%$ and $25 \%$ span. In this region, the secondary velocities have similar spanwise and pitchwise components since the relative locations of the vortices are not changed noticeably. The secondary velocities also indicate that free-stream fluid is continuously convected into the secondary loss core, shown by the red arrows in Figure 8.9(a), thereby producing additional shearing losses downstream. Closer to the endwall (below 5\% span), both SL2F and SL2C show large pitchwise secondary velocities induced by the passage vortex. The effects of the counterrotating corner vortex on the secondary velocities in this region, although small, can be seen between 0 and $20 \%$ pitch for SL2C. Finally, the pitchwise mass-averaged results in Figure 8.9(b) confirm the qualitative assessment of the contour plots: contouring greatly reduces the secondary kinetic energy, mainly associated with a diminished passage vortex.

\subsubsection{Downstream Growth of Secondary Losses}

Additional measurements were collected further downstream of the cascades to document the progression of the secondary flows and determine the mixing losses at the design Mach number. Figure 8.10(a) shows colour floods of $C_{S K E}$ at $2.0 C_{X}$. 
(a)
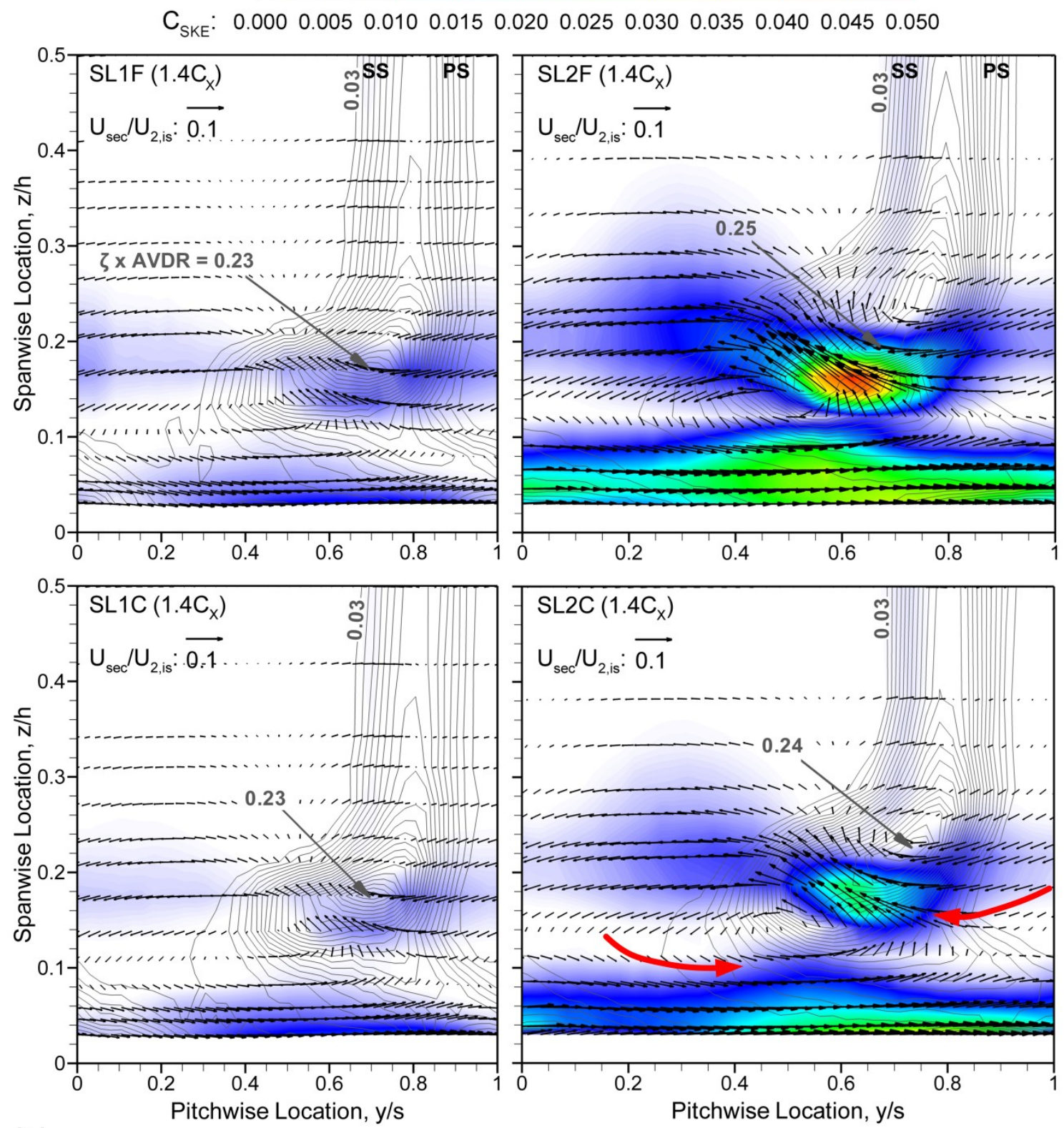

(b)

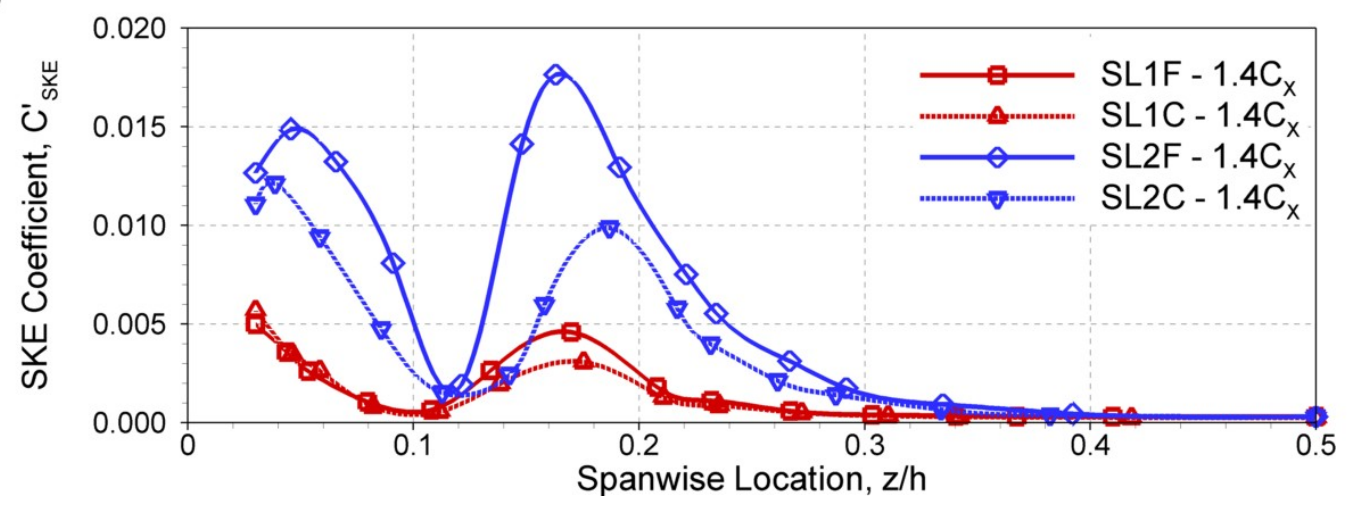

Figure 8.9 (a) Secondary kinetic energy coefficients and secondary velocities and (b) pitchaveraged $C_{S K E}$ at $1.4 C_{X}$ 
(a)

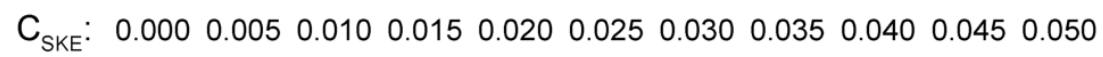
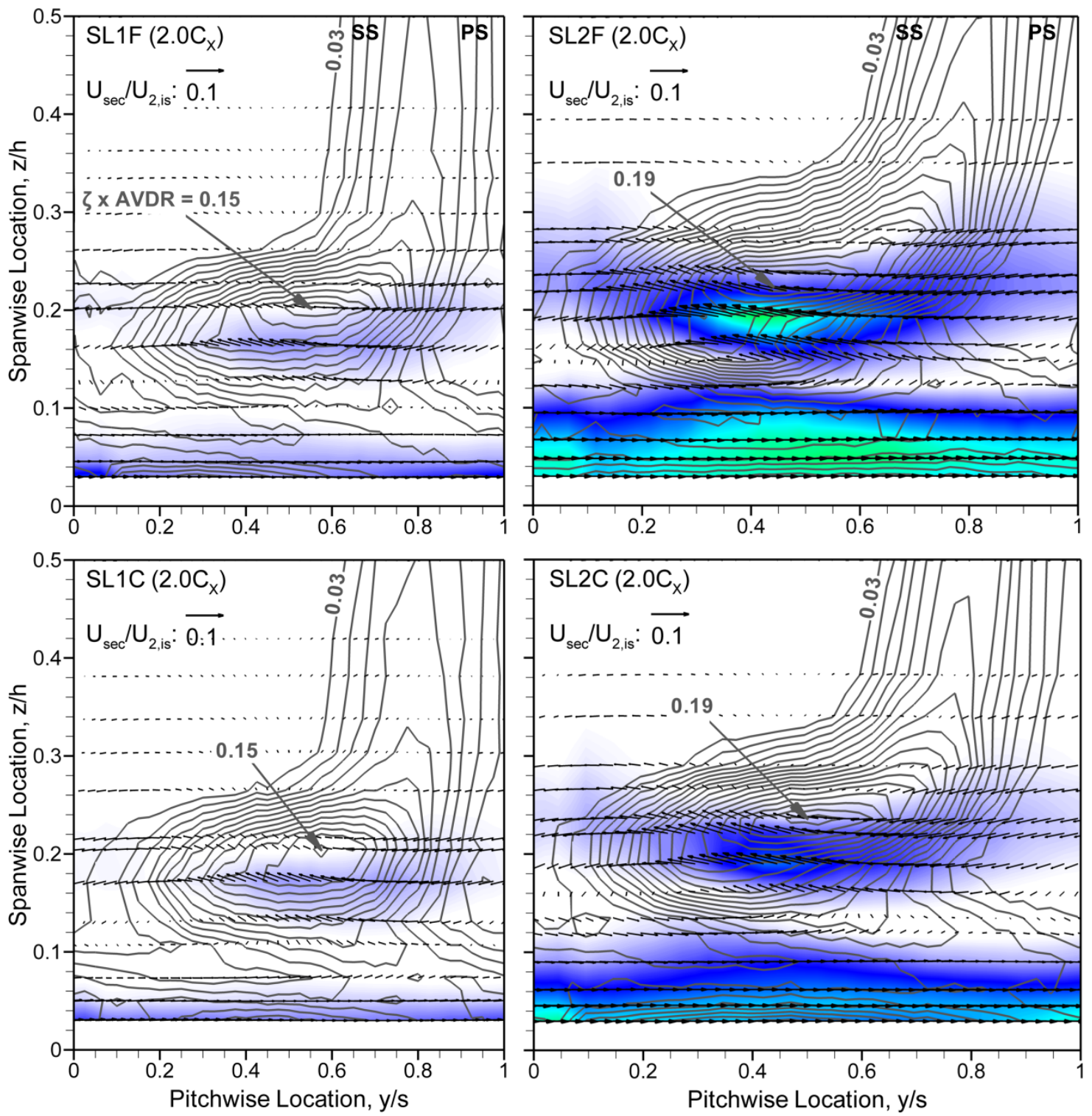

(b)

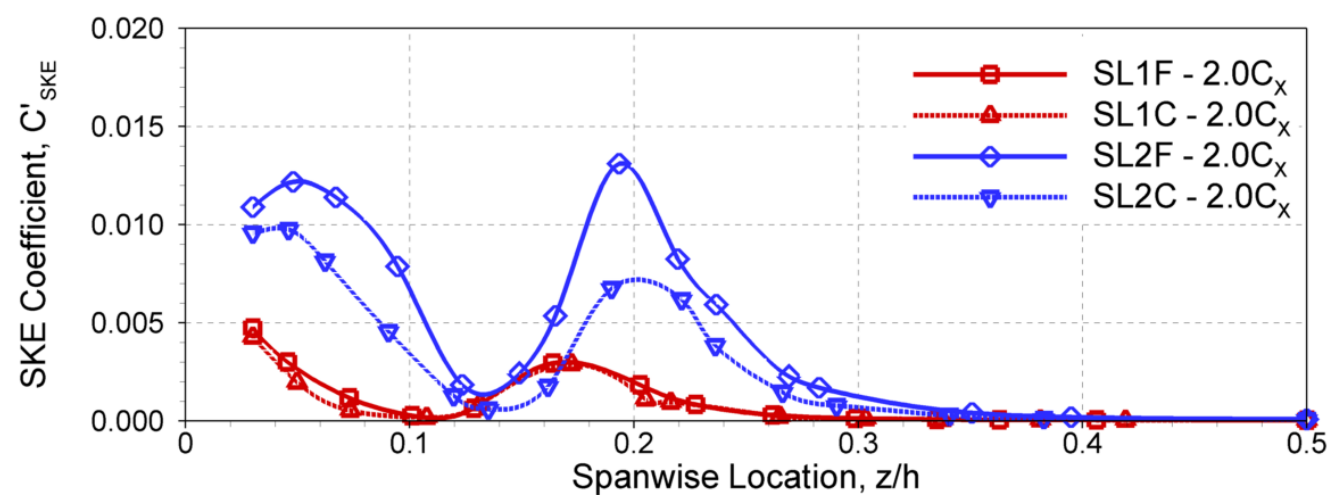

Figure 8.10 (a) Secondary kinetic energy coefficients and secondary velocities and (b) pitchaveraged $C_{S K E}$ at $2.0 C_{X}$ 
Comparing the results in Figure 8.10(a) to those in Figure 8.9(a) indicates significant diffusion and mixing of flow fields downstream of the cascades. The maximum loss values are reduced for all the cases in Figure 8.10(a), but the secondary losses occupy larger regions of the flow fields. There are also marked reductions in the magnitudes of both secondary velocities and $C_{S K E}$.

SL1C produces slightly smaller loss cores and smaller $C_{S K E}$ than SL1F, particularly below 10\% span as shown in Figure 8.10(b). On the other hand, they both show similar $C_{S K E}$ at around $15 \%$ span, indicative of different mixing rates downstream of $1.4 C_{X}$. The mixing is more pronounced downstream of the SL2 cascades: the two loss cores for SL2F in Figure 8.9(a) have merged into one in Figure 8.10(a), the secondary loss penetration depths have increased significantly, and the near-wall losses are mainly associated with the skewed endwall boundary layers below 5\% span. The mixing-out and dissipation of the primary and secondary kinetic energy raise the losses in both cascades, and by $2.0 C_{X}$ SL2C shows smaller losses than SL2F. The difference in loss generation between $1.4 C_{X}$ and $2.0 C_{X}$ for SL2F and SL2C is primarily due to the differences in the intensities of the vortical structures at $1.4 C_{X}$ (Figure 8.8(a)) and the associated $C_{S K E}$ (Figure 8.9(a)). In Figure $8.10(\mathrm{a})$, the secondary velocities from $10 \%$ to $25 \%$ span have smaller spanwise components than those in Figure 8.9(a), suggesting greater spanwise mixing rates (than pitchwise) between the two planes. The secondary velocities below $10 \%$ span, having

primarily pitchwise components, are also significantly smaller due to weaker crossflows at the endwalls. Figure 8.10(b) depicts the spanwise migration of the secondary flows with peak $C_{S K E}^{\prime}$ values displaced towards midspan. Overall, the application of contouring positively influences the flow field in terms of both loss and SKE reductions for SL2C. However, the flow non-uniformities at $2.0 C_{X}$ indicate additional mixing losses yet to come. The fully mixed-out losses will be discussed in Section 8.5.

\subsection{Computational Results at the Design Mach Number}

\subsubsection{Introduction}

The CFD simulations were performed following the completion of the experiments, using the measured boundary conditions at the design outlet Mach number $\left(M_{2, i s}=0.78\right)$ and Reynolds number $\left(\operatorname{Re}_{C, 2}=600,000\right)$. The results from fully-turbulent simulations 
with the Shear Stress Transport turbulence model are presented here. Further details regarding the computations may be found in Chapter 5.

\subsubsection{Downstream Flow Field Predictions at $1.4 C_{X}$}

Figure 8.11 shows the predicted streamwise vorticity coefficients and mass-weighted energy loss coefficients at the design Mach number at $1.4 C_{X}$. The application of contouring reduces the vorticity coefficients for both cases, although the reduction is more pronounced for SL2C.

There are significant differences between the numerical results in Figure 8.11 and the measurements in Figure 8.8(a). CFD in general predicts stronger vortical structures and larger losses, indicative of lower diffusion rates and higher dissipation rates in the computations. In Figure 8.11, SL1C shows a weaker passage vortex than SL1F, whereas the counter and corner vortices remain nearly unaffected. Accordingly, the loss core associated with the passage vortex at around $17 \%$ span is also smaller for SL1C. The experimental results, on the other hand, show very similar vortical structures and losses for SL1F and SL1C in Figure 8.8(a). The additional loss cores in the computations at about $10 \%$ span, coincident with a region of negative vorticity, do not appear in the experiments either.

The numerical results show substantial benefits from contouring for SL2C, as compared to SL2F: the passage and counter vortices are smaller and less intense, and the loss cores are significantly reduced in size and peak magnitude. The numerical results also show small reductions in both corner vortex intensity, and near-wall losses below $5 \%$ span. The measurements in Figure 8.8(a), however, do not confirm the CFD predictions: CFD overpredicts the loss reduction due to contouring and does not capture the corner vortex intensification for SL2C.

The pitchwise mass-averaged results from the experiments and the CFD are presented in Figure 8.12 and Figure 8.13. As anticipated from the vorticity plots, CFD predicts reductions in both underturning and overturning: the application of endwall contouring yields more uniform flow fields downstream of the cascades. The predicted exit flow angles are generally in good agreement with the measurements for the contoured cascades, in particular for SL2C. The predictions for SL2F, on the other hand, show 
larger flow underturning $\left(-2^{\circ}\right)$ and higher spanwise migration of the secondary flows at $1.4 C_{X}(+0.05 \mathrm{z} / \mathrm{h})$ than measured. SL2F shows a larger reduction in overturning near the endwall $(0<z / h<0.03)$ than SL2C due to the presence of a stronger corner vortex in the simulations.
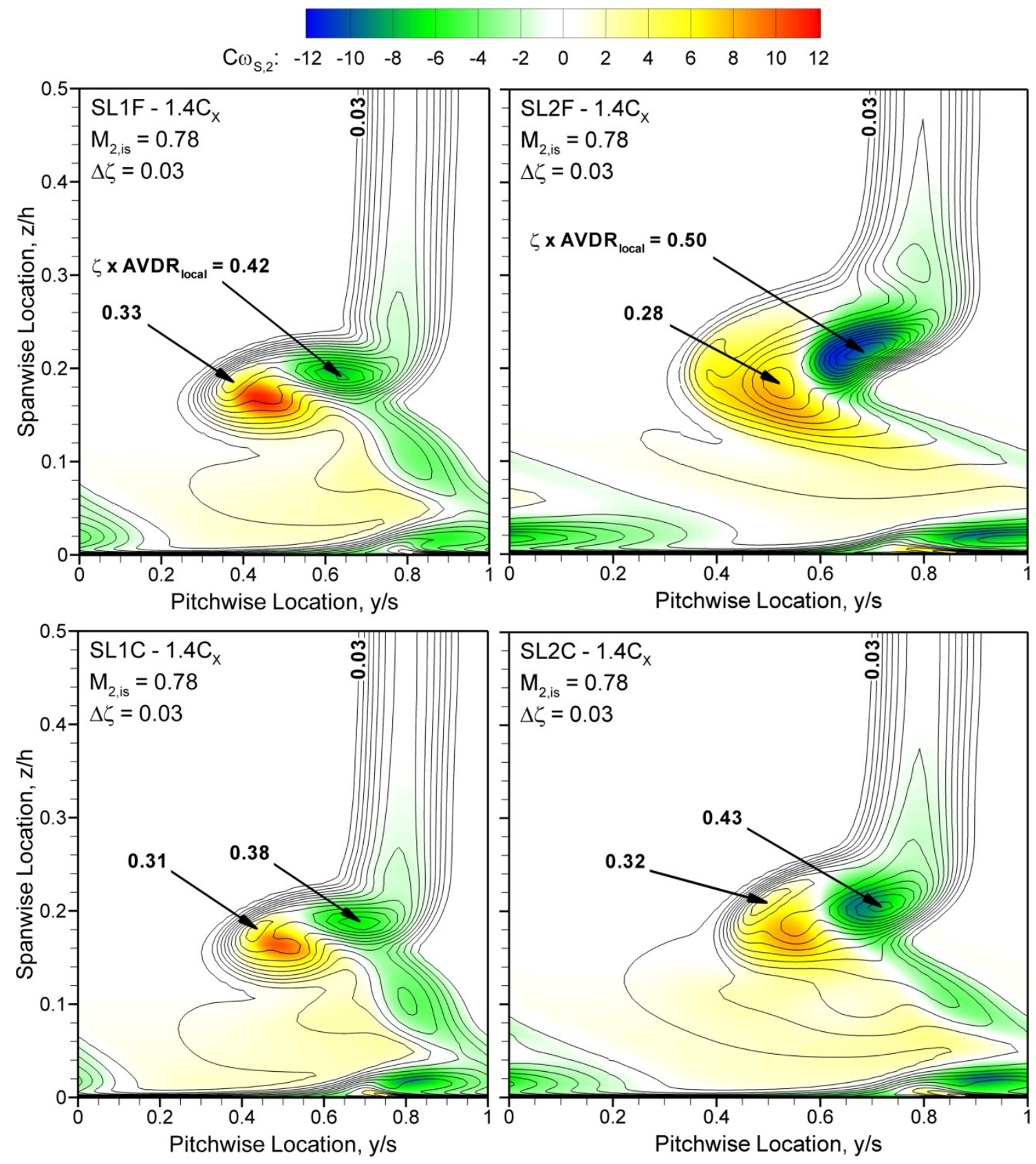

Figure 8.11 CFD predictions for SL2F and SL2C: colour floods of streamwise vorticity coefficient overlaid on contour lines of mass-weighted energy loss coefficient $\left(M_{2, i s}=0.78\right)$ 

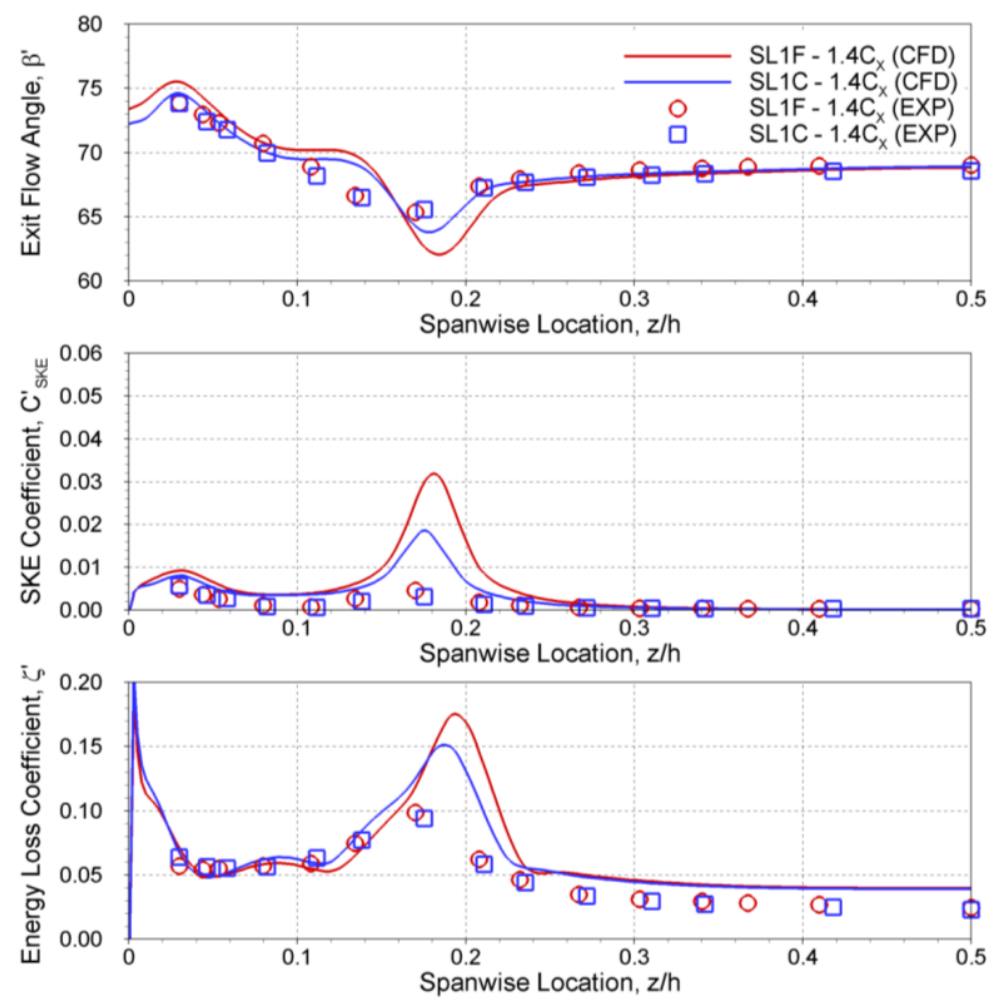

Figure 8.12 Experimental and numerical pitch-averaged results for SL1F and SL1C at $1.4 C_{X}$ : exit flow angles, SKE coefficients and energy loss coefficients $\left(M_{2, i s}=0.78\right)$
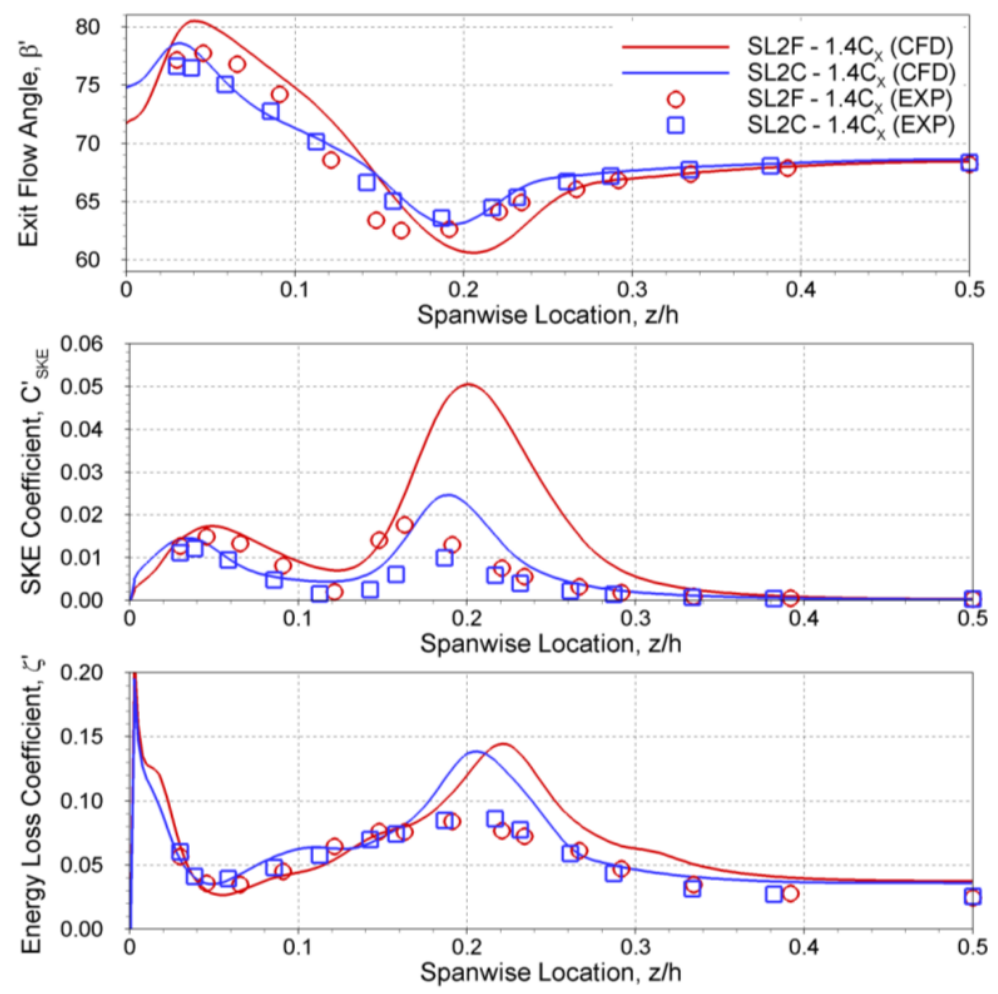

Figure 8.13 Experimental and numerical pitch-averaged results for SL2F and SL2C at $1.4 C_{X}$ : exit flow angles, SKE coefficients and energy loss coefficients $\left(M_{2, i s}=0.78\right)$ 
The CFD results also show benefits for endwall contouring in terms of reductions in both $C_{S K E}^{\prime}$ and energy loss coefficients. This is particularly evident for SL2C, which shows a significant drop in $C_{S K E}^{\prime}$ at $20 \%$ span. However, the loss reductions are more modest. The present fully-turbulent numerical results indicate mass-averaged row loss reductions of $5 \%$ for SL1C, as compared to SL1F, and 10\% for SL2C, as compared to SL2F. Overall, CFD predicts higher $C_{S K E}^{\prime}$ and energy loss coefficients than the experiments, which is consistent with the findings in Chapter 7. The experimental results are summarized next.

\subsection{Integrated Results at both Design and Off-Design Mach Numbers}

The pitchwise mass-averaged energy loss coefficients at the design Mach number are shown in Figure 8.14.

(a)

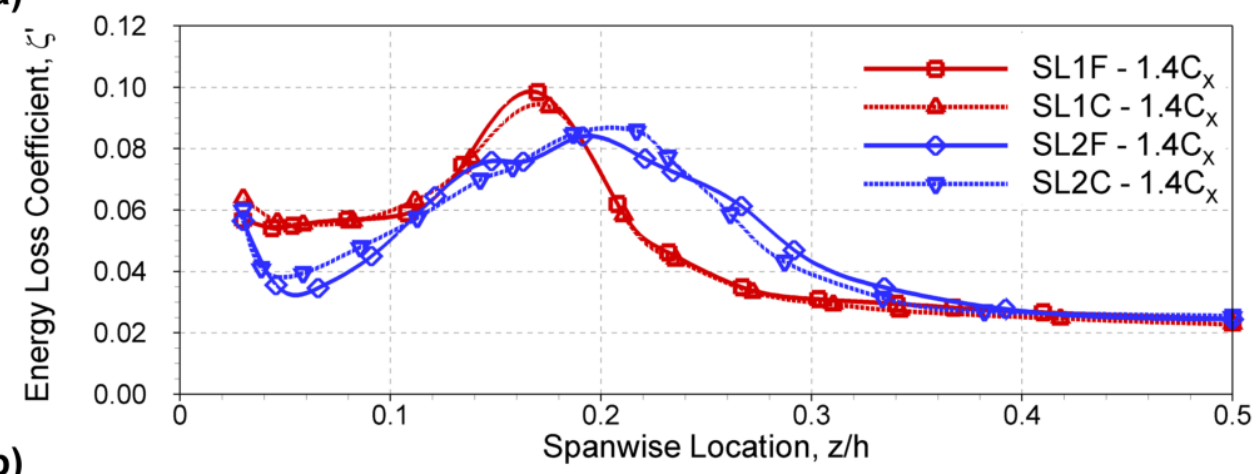

(b)

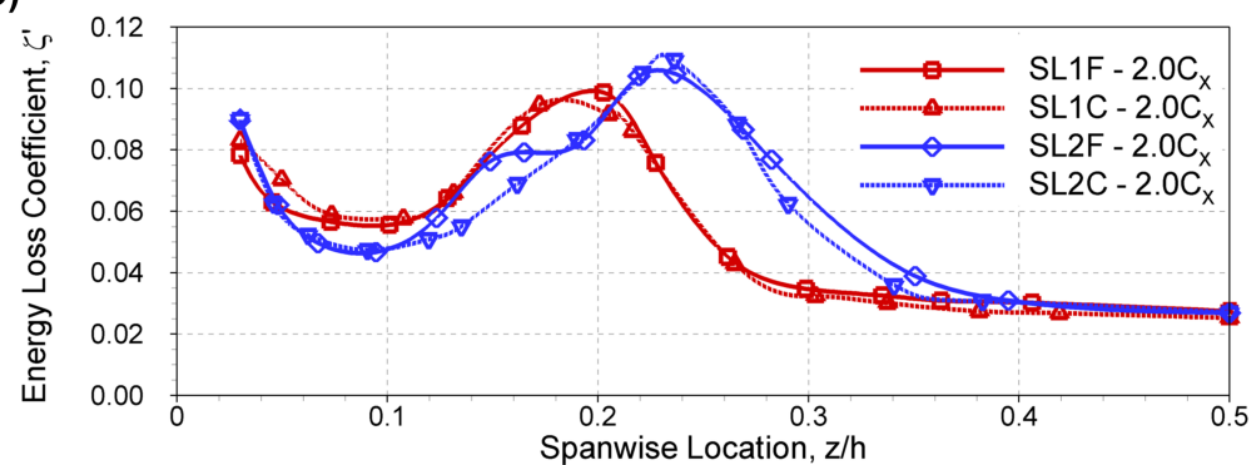

Figure 8.14 Pitch-averaged energy loss coefficients: (a) $1.4 C_{X}$ and (b) $2.0 C_{X}$

As anticipated from the contour plots, SL1C shows slightly smaller losses than SL1F, associated with less intense passage and counter vortices. The secondary losses increase downstream of the cascades, and the contributions of the near-wall losses below $10 \%$ 
span become more significant due to reduced overturning, and the resulting larger mass fluxes. At $2.0 C_{X}$, SL1C shows higher losses than SL1F from 3\% to $7 \%$ span due to larger $S K E$ dissipation between $1.4 C_{X}$ (Figure 8.9(a)) and 2.0 $C_{X}$ (Figure 8.10(a)). Like the SL1 cascades, the SL2 cascades produce similar row losses at $1.4 C_{X}$, although there are some differences in the spanwise distributions. By 2.0 $C_{X}$, SL2C has lower losses than SL2F in two distinct areas at $12 \%$ to $18 \%$ span, corresponding to the passage vortex, and at $27 \%$ to $38 \%$ span, where the counter vortex is located. The SL2 results, however, show very similar losses from $3 \%$ to $10 \%$ span: the application of contouring does not increase the near-wall losses for SL2C, unlike the case of SL1C.

The overall-integrated results, from 0 to $50 \%$ span, at the design Mach number, both mass-averaged and mixed-out, are shown in Table 8.2. The inlet endwall boundary-layer losses are subtracted from the downstream results, and therefore the comparisons are based on actual row losses. The mixed-out results include additional losses due to primary and secondary kinetic energy dissipation, as well as changes in static pressure (e.g. pressure recovery). On the other hand, the mixing-out calculations does not account for the additional loss production on the endwalls downstream of the measurement planes. Consequently, the mixed-out values from $2.0 C_{X}$ are larger than those from $1.4 C_{X}$. The additional losses, however, do not affect the conclusions regarding the effectiveness of endwall contouring. SL1F and SL1C produce similar mass-averaged row losses at both measurement planes despite the small differences in $C^{\prime \prime}{ }_{S K E}$ at $1.4 C_{X}$. The results at $2.0 C_{X}$ display about a $30 \%$ reduction in $C^{\prime \prime}{ }_{S K E}$ and a $20 \%$ increase in row losses for the SL1 cascades. The mixed-out values are also very similar for the two cascades, although the additional mixing losses from $2.0 C_{X}$ are smaller than those from $1.4 C_{X}$. This is attributed to the more uniform flow fields at $2.0 C_{X}$. Notwithstanding the small differences, it may be concluded that the collective effects of endwall contouring for SL1C, which may appear aggressive according to Ingram et al. (2005b), do not adversely affect the aerodynamic performance. However, the measurements show very similar losses, and therefore do not validate the $5 \%$ reduction in predicted mass-averaged row losses by CFD. 
Table 8.2 Overall integrated results for SL1F and SL1C at the design Mach number

\begin{tabular}{|c|c|c|c|c|c|c|c|c|}
\hline & \multicolumn{4}{|c|}{$1.4 C_{x}$} & \multicolumn{4}{|c|}{$2.0 C_{X}$} \\
\hline & SL1F & SL1C & SL2F & SL2C & SL1F & SL1C & SL2F & SL2C \\
\hline \multicolumn{9}{|l|}{ Mass-Averaged } \\
\hline SKE Coefficient, C" SKE & 0.0014 & 0.0012 & 0.0056 & 0.0035 & 0.0010 & 0.0008 & 0.0042 & 0.0027 \\
\hline Energy Loss Coefficient, $\zeta$ & 0.035 & 0.035 & 0.036 & 0.036 & 0.042 & 0.041 & 0.048 & 0.045 \\
\hline Profile Energy Loss Coefficient, $\zeta_{P}$ & 0.024 & 0.023 & 0.024 & 0.024 & 0.027 & 0.025 & 0.026 & 0.025 \\
\hline Secondary Energy Loss Coefficient, $\zeta_{s}$ & 0.011 & 0.012 & 0.012 & 0.012 & 0.015 & 0.016 & 0.022 & 0.020 \\
\hline \multicolumn{9}{|l|}{ Mixed-Out } \\
\hline Energy Loss Coefficient, $\zeta$ & 0.040 & 0.039 & 0.045 & 0.042 & 0.045 & 0.044 & 0.056 & 0.051 \\
\hline Profile Energy Loss Coefficient, $\zeta_{P}$ & 0.025 & 0.023 & 0.025 & 0.025 & 0.027 & 0.025 & 0.026 & 0.026 \\
\hline Secondary Energy Loss Coefficient, $\zeta_{s}$ & 0.015 & 0.016 & 0.020 & 0.017 & 0.018 & 0.019 & 0.030 & 0.025 \\
\hline
\end{tabular}

In contrast to SL1, the SL2 results in Table 8.2 show larger SKE dissipation and higher mixing losses downstream of $1.4 C_{X}$. In particular, the mass-averaged row losses for SL2F have risen by $33 \%$ at $2.0 C_{X}$. SL2C, on the other hand, shows smaller changes between the two planes, as anticipated from Figure 8.9(a) and Figure 8.10(a). Overall, the results at $2.0 C_{X}$ indicate reductions in both mass-averaged (5\%) and mixed-out $(8 \%)$ row losses due to endwall contouring. In an engine environment, the additional benefit associated with reduced $S K E$ and lower under/overturning would be realized in the downstream blade rows due to lower variation in incidence. This is particularly significant for rotating blades, since a given variation in the flow angle of the absolute velocity vector leaving the nozzle might result in an even larger variation in flow angle for the relative velocity seen by the following blade.

In addition to the investigations at the design Mach number, probe measurements were collected at two off-design Mach numbers at $1.4 C_{X}$. The overall-integrated results at both design and off-design Mach numbers are summarized in Table 8.3 and Table 8.4. The effects of Mach number variation on profile losses and secondary losses for flat endwalls are presented in Chapter 6 and Chapter 7, respectively. As discussed there, increasing the outlet Mach number in the experiments is accompanied with larger Reynolds numbers and row velocity ratios, smaller Zweifel coefficients, higher suctionsurface Mach numbers and increase aft loading, all of which affect the losses.

Overall, the losses and secondary kinetic energy coefficients decrease with increasing Mach number. The results in Table 8.3 and Table 8.4 indicate the largest benefit for the 
contoured cascades at the lowest Mach numbers. This is in part attributed to the corresponding change in loading distribution. As discussed in Chapter 7, the airfoils shift from aft to more mid loaded as the Mach number is reduced, and this results in higher secondary losses. Accordingly, endwall contouring becomes more effective in alleviating the transverse pressure gradients in the forward and mid parts of the passage to reduce the secondary losses. Operating at higher Mach numbers, on the other hand, results in increased aft loading, thereby reducing the benefit of contouring. This is especially evident for SL2C in Table 8.4: SL2C produces slightly higher mass-averaged row losses than SL2F at the highest Mach number, but it displays lower mixed-out losses due to the smaller contribution from $S K E$ dissipation (about $40 \%$ lower $C^{\prime \prime}{ }_{S K E}$ than SL2F). Of course, these results only show the effects of varying the exit Mach number for a given airfoil; the results in the present study do not indicate whether compressibility effects decrease or increase the benefit of endwall contouring with increasing Mach number for rows that were optimized for each of these Mach numbers.

Table 8.3 Overall integrated results for SL1F and SL1C at three different Mach numbers

\begin{tabular}{|c|c|c|c|c|c|c|}
\cline { 2 - 7 } \multicolumn{1}{c|}{} & SL1F & SL1C & SL1F & SL1C & SL1F & SL1C \\
\hline Outlet Isentropic Mach Number, $\boldsymbol{M}_{2, \text { is }}$ & \multicolumn{2}{|c|}{0.65} & \multicolumn{2}{|c|}{0.78} & \multicolumn{2}{c|}{0.91} \\
\hline Zweifel Coefficient, $Z \mathbf{w}$ & \multicolumn{2}{|c|}{0.81} & \multicolumn{2}{|c|}{0.75} & \multicolumn{2}{c|}{0.66} \\
\hline Mass-Ave. SKE Coefficient, $\boldsymbol{C}^{\prime \prime}$ sKE & 0.0016 & 0.0014 & 0.0014 & 0.0012 & 0.0014 & 0.0014 \\
\hline Mass-Ave. Row Loss Coefficient, $\boldsymbol{\zeta}$ & 0.037 & 0.036 & 0.035 & 0.035 & 0.032 & 0.033 \\
\hline Mixed-Out Row Loss Coefficient, $\boldsymbol{\zeta}$ & 0.042 & 0.041 & 0.040 & 0.039 & 0.038 & 0.039 \\
\hline
\end{tabular}

Table 8.4 Overall integrated results for SL2F and SL2C at three different Mach numbers

\begin{tabular}{|c|c|c|c|c|c|c|}
\hline & SL2F & SL2C & SL2F & SL2C & SL2F & SL2C \\
\hline Outlet Isentropic Mach Number, $M_{2, \text { is }}$ & \multicolumn{2}{|c|}{0.65} & \multicolumn{2}{|c|}{0.78} & \multicolumn{2}{|c|}{0.91} \\
\hline Zweifel Coefficient, Zw & \multicolumn{2}{|c|}{1.07} & \multicolumn{2}{|c|}{0.97} & \multicolumn{2}{|c|}{0.89} \\
\hline Mass-Ave. SKE Coefficient, C" SKE & 0.0065 & 0.0042 & 0.0056 & 0.0035 & 0.0050 & 0.0031 \\
\hline Mass-Ave. Row Loss Coefficient, $\zeta$ & 0.038 & 0.037 & 0.036 & 0.036 & 0.033 & 0.034 \\
\hline Mixed-Out Row Loss Coefficient, $\zeta$ & 0.048 & 0.045 & 0.045 & 0.042 & 0.041 & 0.040 \\
\hline
\end{tabular}

As a final note, comments have been made recently regarding the suitability of $C_{S K E}$ as the objective function for optimization of $3 \mathrm{D}$ airfoils and endwall contouring (Schuepbach et al., 2009; MacIsaac et al., 2010; Snedden et al., 2010). In the present study, $S K E$ dissipation between $1.4 C_{X}$ and $2.0 C_{X}$ accounts for less than $15 \%$ of the 
additional losses. The majority of the additional losses appear to arise from primary kinetic energy dissipation, including shearing losses at the endwalls, as well as pressure force effects. Furthermore, the mass-averaged results from the experiments do not corroborate the CFD predictions at $1.4 C_{X}$. On the other hand, the mixed-out results from the experiments show improvements due to contouring, particularly for the more highlyloaded SL2C cascade. In view of this, the fully mixed-out energy loss coefficient may be a more appropriate objective function, provided that the designer examines the flow field to confirm the benefits for all other parameters that may influence the overall performance (e.g. TKE and under/overturning). 


\section{Chapter 9}

\section{Modification of the Airfoil Pressure Surface for Secondary Loss Reduction}

\subsection{Introduction}

The importance of secondary loss reduction to the overall stage efficiency was highlighted in the preceding chapters. Non-axisymmetric endwall contouring has been demonstrated to be a viable option for mitigating the secondary flows in turbines. An alternative method for secondary loss reduction that does not involve modifications to the endwall is investigated in the present chapter. Instead, the airfoil geometry is adjusted to modify the static pressure distribution near the endwall and thereby reduce the secondary losses, without altering the overall blade loading. A related study was recently completed by Knezevici (2011), who demonstrated significant secondary loss reduction for a modified low-speed cascade, designated PAKD-FP.

The SL2F cascade was redesigned for the present study, with the new designation SL2P. This chapter describes the design philosophy of SL2P, presents CFD results to assess the predicted loss generation for SL2F and SL2P, and then presents corresponding experimental results. The discussion is primarily focused on the design point, $M_{2, i s}=0.8$. The integrated results at the design Mach number and at two off-design Mach numbers are summarized at the end.

\subsection{Concept for the Modified Pressure Side}

\subsubsection{Design Approach}

One of the chief geometric features of the contoured endwalls described in Chapter 8 is the pressure-side protrusion in the forward part of the passage, referred to as $\mathrm{P}_{1}$. This 
region of the flow is typically near stagnation in cascades with flat endwalls. The low momentum of the fluid in the near-stagnation region causes the fluid to be strongly driven across the passage by the blade-to-blade pressure difference. This fluid feeds into the pressure-side leg of the horseshoe vortex to enhance its size and strength. As discussed, the resulting composite vortex is known as the passage or secondary vortex as it reaches the passage outlet. The greater the extent of the near-stagnation region, the more fluid will be contributed to the passage vortex in this way. The protrusion $\mathrm{P}_{1}$ acts to reduce the static pressure on the forward pressure side. The consequent higher streamwise momentum of the fluid results in less fluid being fed into the passage vortex. This reduces its size and strength, and the associated $S K E$ and losses. The other protrusions and depressions incorporated in the endwall contouring have further beneficial effects on the endwall flow elsewhere in the passage, as described in Chapter 8. However, the benefits of the $\mathrm{P}_{1}$ protrusion can also be obtained by simply modifying the geometry of the airfoil on the forward pressure side in such a way that the extent of the near stagnation region is minimized. This is the essence of the pressure-side modification that is investigated in this chapter. The concept was developed by Pratt \& Whitney Aircraft and is the subject of two patent application filings (Patent Application Numbers: US 2007/0258818 A1 and US 2007/0258819 A1).

The modified airfoil examined here was developed from the SL2F geometry and is designated SL2P. The geometries are compared in Figure 9.1. The modified pressure distribution was obtained by increasing the airfoil thickness, particularly near the leading edge, to accelerate the flow along the pressure surface. Other geometric and aerodynamic parameters such as axial chord, pitch spacing and inlet and outlet flow angles are left unchanged to obtain the same row velocity ratio and Zweifel coefficient as for SL2F.

It should be noted that the large increase in the thickness of the airfoil is not an inherent consequence of the pressure side modification. In this case, it is partly the result of retaining the suction-side geometry from the SL2F airfoil. An airfoil designed with the pressure side modification from the start could probably be made considerably thinner. On the other hand, an increased thickness-to-chord ratio may be beneficial in certain situations, such as for cooled turbine blades. The pressure side modification 
could also be confined to the hub, and perhaps tip, regions of the airfoil, with a thinner profile such as the SL2F geometry being applied elsewhere. Other potential consequences of this novel technology, such as increased heat transfer and vibration and reduced part life, will be assessed prior to engine implementation.

\subsubsection{Predicted Airfoil and Endwall Isentropic Mach Number Distributions}

Figure 9.1 shows the predicted and measured loading distributions for the two airfoils. It can be seen that apart from the necessary stagnation point, the static pressure has been lowered substantially over a significant portion of the pressure side of SL2F, as reflected in the raised isentropic Mach numbers. Although the two airfoils have identical suction-side geometries, the changes on the pressure side have also affected the suction side flow: since both blade rows have the same spacing and are turning the flow through the same angle, and thus have the same overall aerodynamic loading, the Mach numbers have increased slightly on the suction side as well.
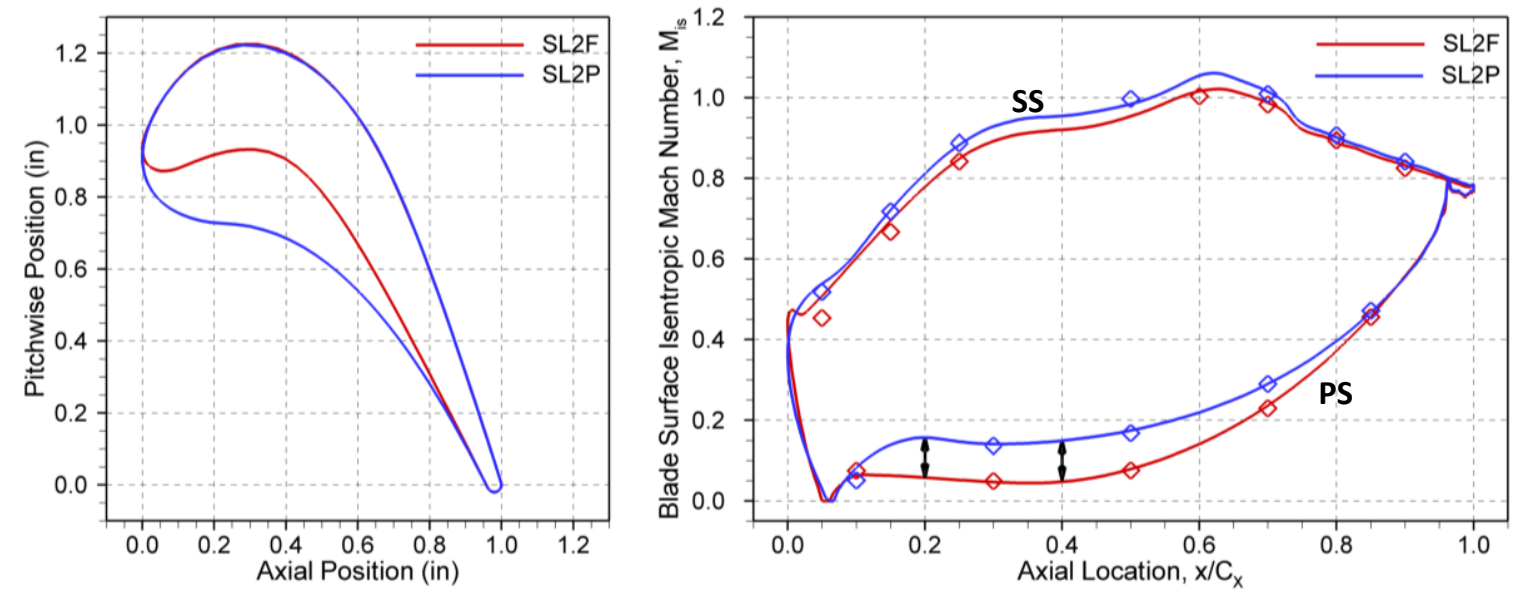

Figure 9.1 Airfoil geometries and midspan loading distributions $\left(M_{2, i s}=0.78\right)$

The predicted endwall isentropic Mach number distributions, based on the inlet centreline total pressure and the wall static pressures, are shown in Figure 9.2. Also shown are the velocity vectors at $1 \%$ span. As indicated in Chapter 8 , the velocity vectors at $0.5 \%$ and $1.5 \%$ span show very similar behaviour to the results presented here. The most striking feature is the significant increase in endwall isentropic Mach numbers near the pressure surface for SL2P. Accordingly, the velocity vectors in the forward part of the passage (enclosed in dashed circles) appear to be more closely aligned in the 
streamwise direction for SL2P. These results are consistent with the endwall flow visualization results of Brear et al. (2002) for their thickened airfoil profile.

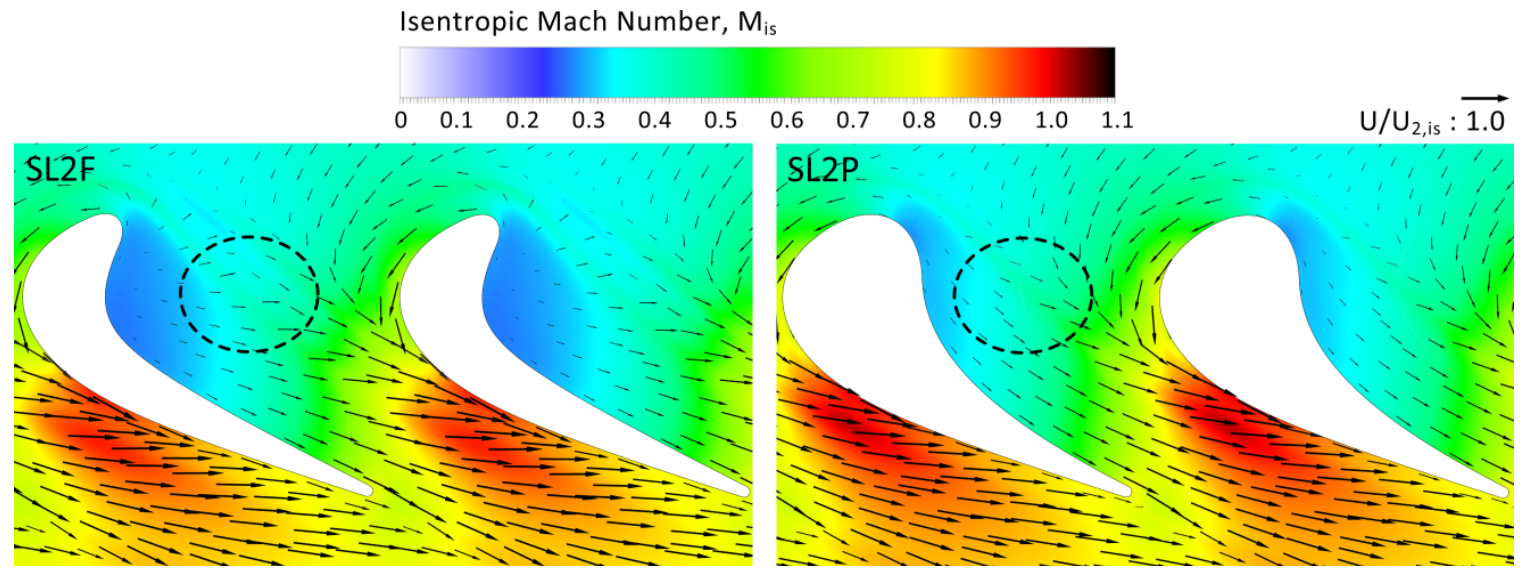

Figure 9.2 CFD predictions: endwall isentropic Mach number distributions overlaid on velocity vectors at $1 \%$ span

\subsection{Experimental Results at the Design Mach Number}

\subsubsection{Surface Flow Visualizations}

The flow visualization results for SL2F and SL2C were examined in Chapter 8. In general, the application of endwall contouring leads to more favourable (streamwiseoriented) mid-passage crossflows and weaker interactions between the vortical structures and the SS boundary layers. The aim of this section is to describe the differences between SL2F and SL2P at the design Mach number.

The suction surface and endwall flow visualization results are presented in Figure 9.3 and Figure 9.4, respectively. The nomenclature of Sieverding (1985) is used as in the earlier chapters: dashed lines are used for flow separation, dotted lines for flow reattachment and arrows for surface shear stress vectors. In Figure 9.3, there is evidence of small SS separation bubbles for both SL2F and SL2P. Following the reattachment, at $\mathrm{R}_{5}$, there is also evidence of flow separation near the trailing edge, as shown by $\mathrm{S}_{6}$. SL2P shows a larger near two-dimensional flow region than SL2F upstream of the separation bubble. As discussed in Chapter 6, SL2P produces about 10\% higher mixed-out profile losses than SL2F.

$\mathrm{S}_{2 \mathrm{~S}}$, marking the suction-side leg of the horseshoe vortex in Figure 9.3, is located closer to the endwall for SL2P. The suction-side leg of the horseshoe vortex is typically 
lifted off the endwall by the action of the passage vortex, marked by $\mathrm{S}_{4}$. The closer proximity of $\mathrm{S}_{2 \mathrm{~S}}$ to $\mathrm{S}_{4}$ for SL2P is perhaps indicative of the weaker influence of the passage vortex. The low shear-stress region between the separation lines is associated with the convection of inlet boundary layer fluid through the passage; the weaker interaction between the passage vortex and the SS boundary layer also reduces the associated mixing losses. The results in Figure 9.3 indicate slightly lower secondary flow penetration depth for SL2P as judged from $\mathrm{S}_{4}$, which is also indicative of a smaller passage vortex.

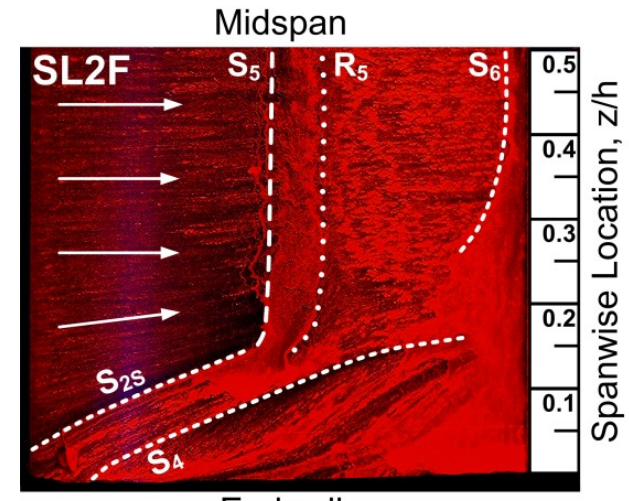

Endwall

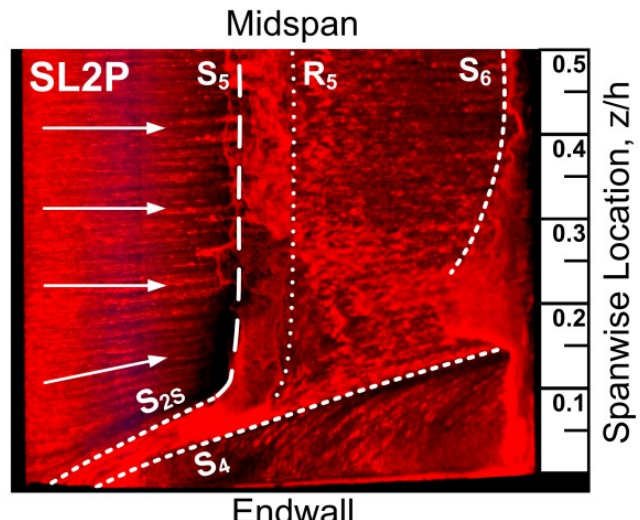

Endwall

Figure 9.3 Suction surface flow visualization results

The endwall flow visualization in Figure 9.4(a) and Figure 9.4(b) show subtle differences between SL2F and SL2P. In comparison to SL2F, $\mathrm{A}_{1}$ is positioned closer to the airfoil leading edge for SL2P, and $\mathrm{S}_{1 \mathrm{P}}$ is shifted towards the pressure side of the passage. The closer proximity of the primary separation line to the pressure surface for SL2P is due to the smaller transverse pressure gradients in the forward part of the passage; this delays the trajectory and growth of the pressure-side leg of the horseshoe vortex. Downstream of $\mathrm{S}_{2 \mathrm{P}}$, the endwall streamlines are oriented more closely in the streamwise direction as indicated by the arrows. Combined with the smaller endwall area for SL2P, the orientation of the endwall crossflows is expected to reduce the entrainment of the endwall boundary layer fluid by the passage vortex. The corner vortex separation line, shown by $\mathrm{S}_{3}$, is also positioned closer to the blade suction surface in Figure 9.4(b), suggesting a smaller corner vortex for SL2P. The associated vortical structures and losses are described next. 

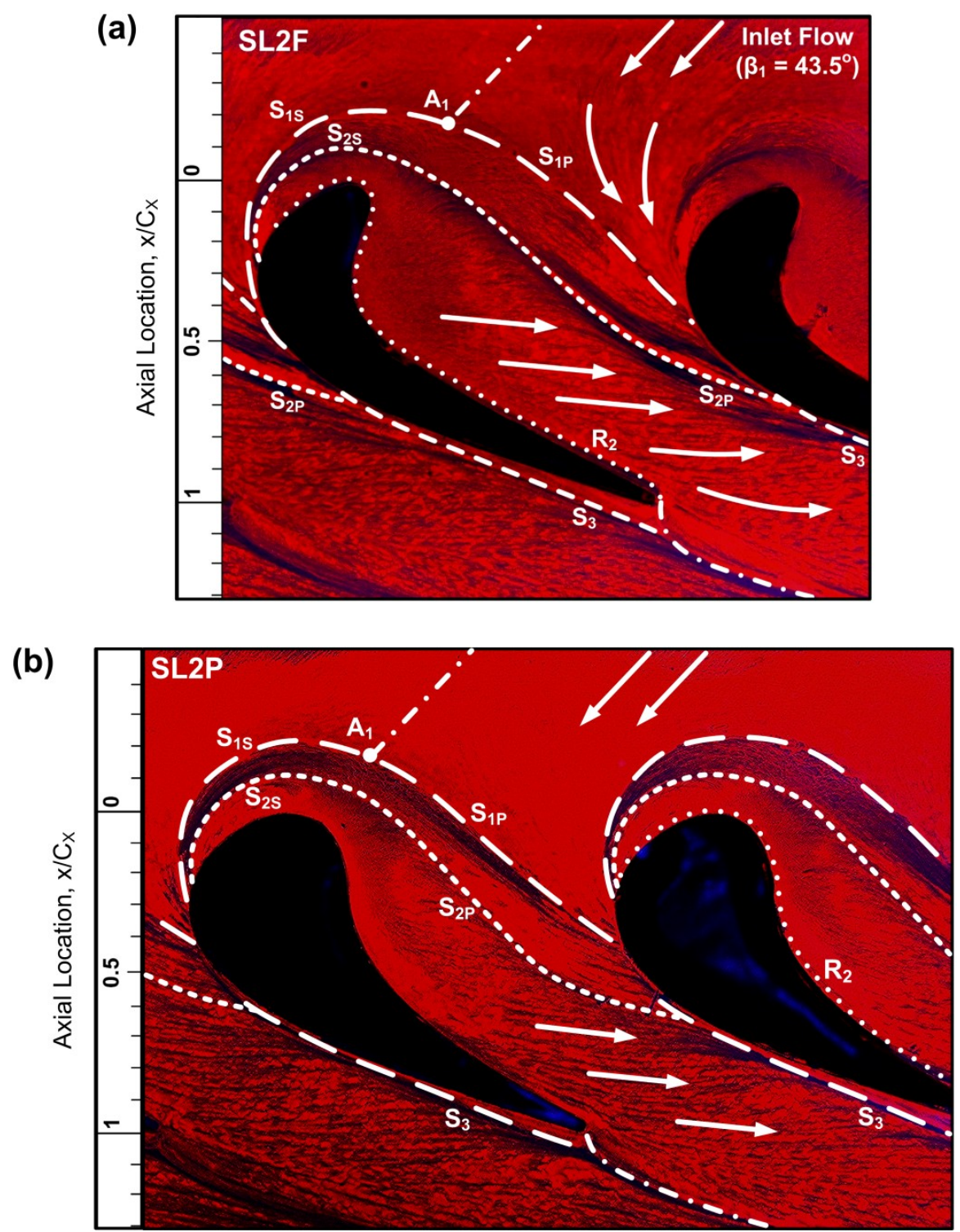

Figure 9.4 Endwall surface flow visualization results: (a) SL2F and (b) SL2P

\subsubsection{Downstream Flow Field Measurements at $1.4 C_{X}$}

Colour flood contour plots of streamwise vorticity coefficient superimposed over contour lines of mass-weighted energy loss coefficient are presented in Figure 9.5(a). SL2P has a wider wake, extending from the midspan to about $30 \%$ span, than SL2F. The reduction in secondary flow penetration depth for SL2P is in agreement with the flow visualization results in Figure 9.3. Secondary losses occupy a smaller portion of the flow field for SL2P with only one distinct loss core, but with a slightly higher peak value: 0.28 for SL2P versus 0.25 for SL2F. Below 10\% span, the contribution of the near-wall losses is attenuated particularly for SL2F, as compared to SL2P, due to lower mass fluxes. 
(a)
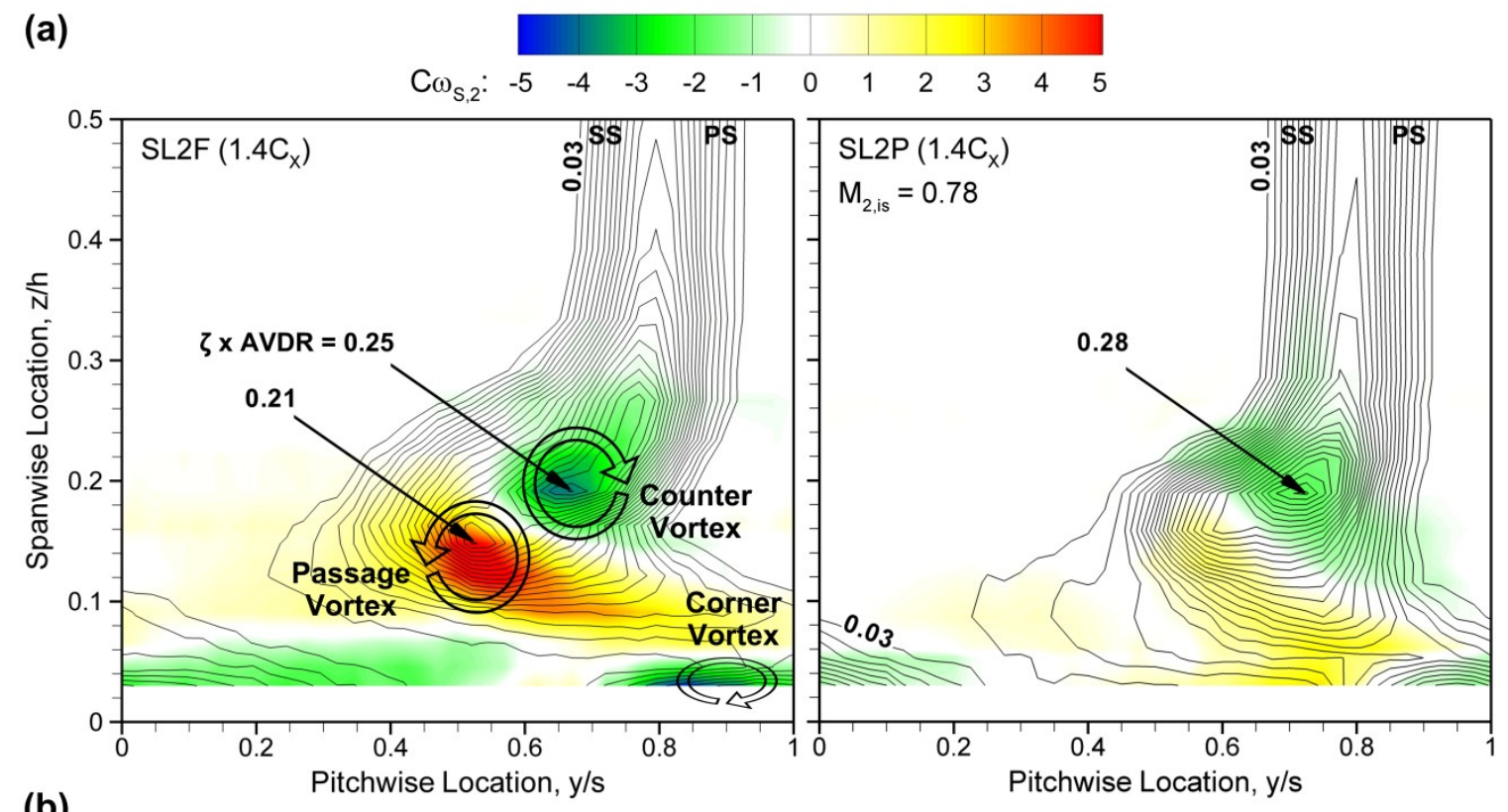

(b)

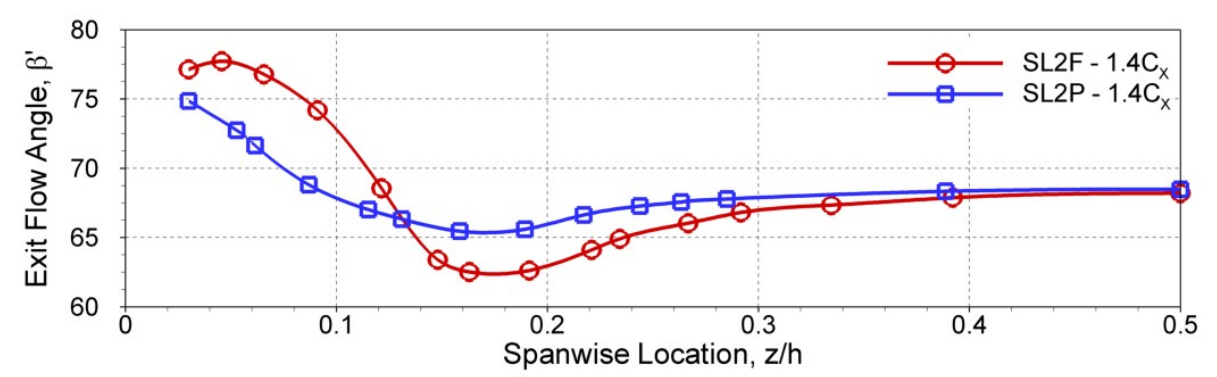

Figure 9.5 (a) Streamwise vorticity coefficients overlaid on contour lines of mass-weighted energy loss coefficient and (b) pitch-averaged exit flow angles at $1.4 C_{X}$

SL2F generates significantly stronger vortical structures than SL2P. The counter vortices are positioned at similar spanwise locations (about 19\% span) for both cascades. The counter vortex is slightly larger for SL2P, extending from about 55\% to $90 \%$ pitch, but considerably less intense. In each case, the centre of the counter vortex coincides with the location of the highest loss core. For SL2F, the loss coefficient contour lines are distorted under the action of the stronger counter vortex from about $15 \%$ to $25 \%$ span. Therefore, the secondary losses penetrate further into the inviscid flow region for SL2F, potentially resulting in higher mixing losses. SL2F also exhibits a very intense passage vortex, centred at approximately 55\% pitch; the passage vortex further distorts the loss coefficient contour lines from $5 \%$ to $20 \%$ span, and augments the mixing downstream of the measurement plane. In contrast, SL2P displays lower levels of positive vorticity from about $3 \%$ to $18 \%$ span. The significant reduction in positive vorticity for SL2P is 
accompanied by relatively undisturbed loss coefficient contour lines. Near the endwall, SL2P displays a smaller and less intense counter-rotating corner vortex, which is consistent with the endwall flow visualization results in Figure 9.4. The reductions in negative vorticity, associated with the counter and corner vortices, are in part due to the reduction in passage vortex intensity: the smaller passage vortex for SL2P has a weaker interaction with the suction-surface boundary layer, which in turn reduces the negative vorticity production. The improved alignment of the near-wall streamlines in the streamwise direction for SL2P, seen in Figure 9.4(b), reduces the endwall boundary layer migration into the corner vortex.

The pitch-averaged exit flow angles in Figure 9.5(b) indicate very similar total flow turning for both cascades at midspan. SL2P shows smaller flow underturning at about $17 \%$ span and smaller flow overturning near the endwall. The significant reduction in under/overturning is primarily associated with the smaller and less intense passage vortex for SL2P. Although not shown here, the smaller variation in exit flow angles is also accompanied with more uniform mass flux distribution for SL2P.

Figure 9.6(a) shows colour floods of secondary kinetic energy coefficient $\left(C_{S K E}\right)$, overlaid on secondary velocity vectors. The pitchwise mass-averaged values of $C_{S K E}$ are also shown in Figure 9.6(b). Overall, SL2P has smaller secondary velocities and lower $C_{S K E}$ than SL2F. The reduction in secondary velocities is particularly evident in the interaction region between the passage vortex and the counter vortex, consistent with the results for streamwise vorticity, shown in Figure 9.5(a). Below 5\% span, larger secondary velocities are induced by the action of the passage vortex, resulting in a considerable rise in $C^{\prime}{ }_{S K E}$ for SL2P. These results suggest that there will be significant differences in the losses due to the downstream mixing for SL2F and SL2P associated with the dissipation of the remaining $S K E$.

The pitchwise mass-averaged energy loss coefficients are presented in Figure 9.6(c). SL2P generates higher losses than SL2F from about 3\% to $12 \%$ span due to the closer proximity of the secondary flows to the endwall and the larger mass fluxes in this region (smaller overturning shown in Figure 9.5(b)). SL2F, on the other hand, generates higher losses from $20 \%$ to $35 \%$ span, associated with the stronger passage and counter vortices in Figure 9.5(a). The overall integrated results are summarized in section 9.5. 
(a)

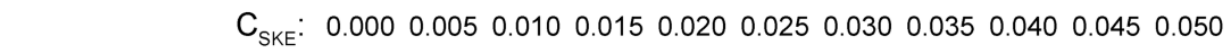
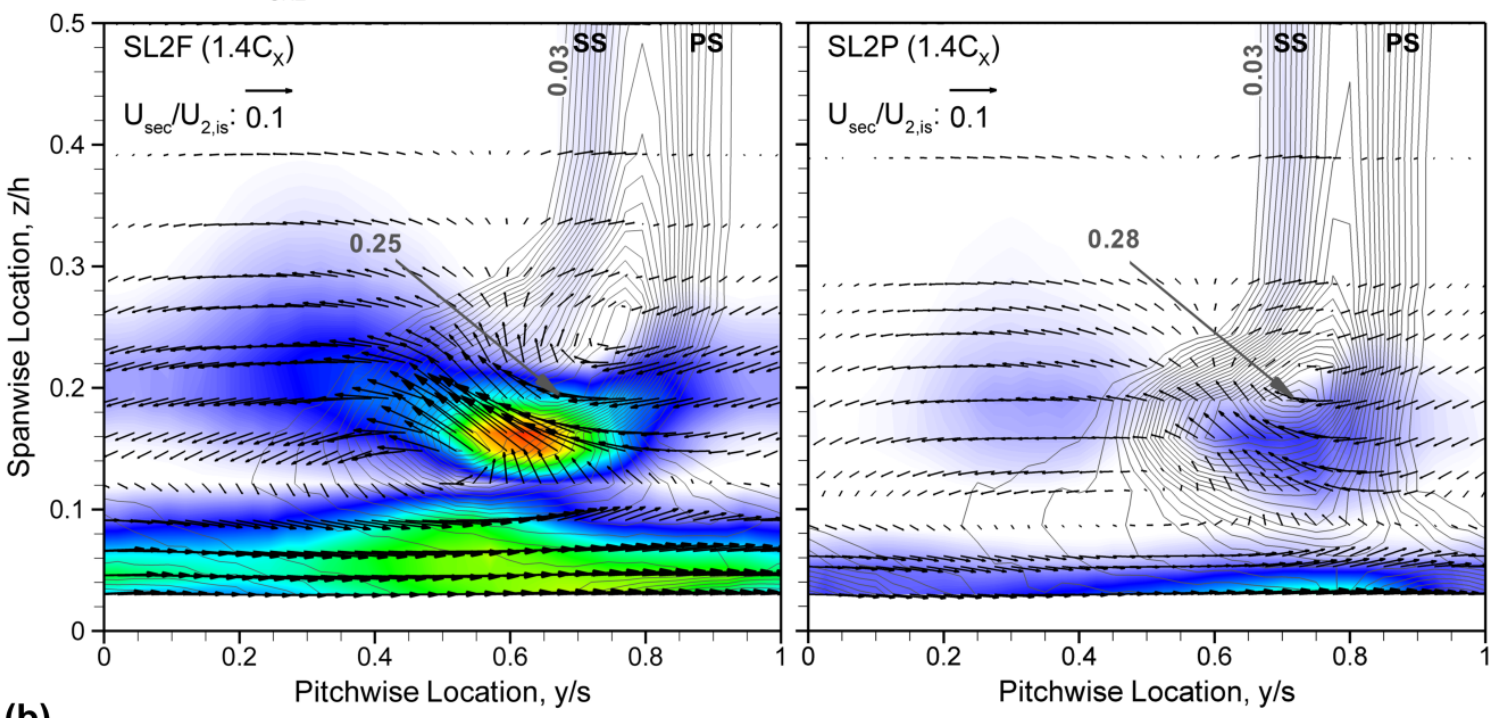

(b)

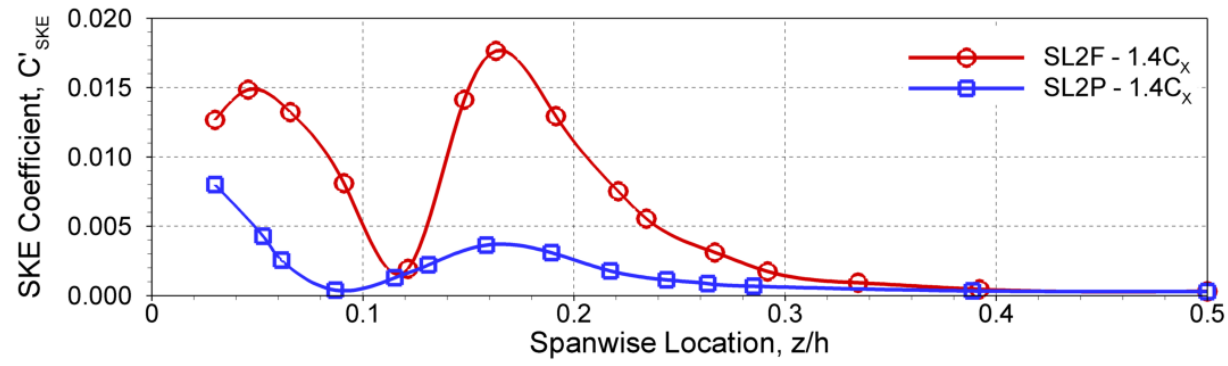

(c)

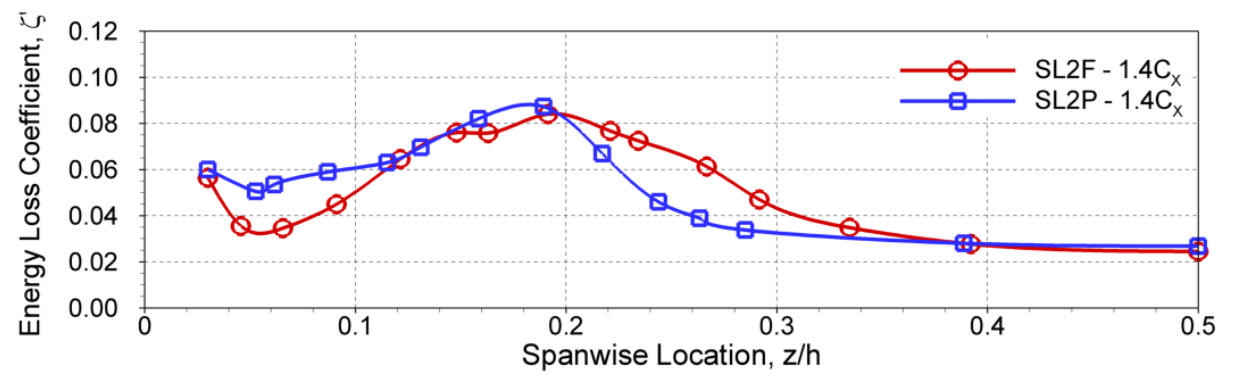

Figure 9.6 (a) Secondary kinetic energy coefficients and secondary velocities, (b) pitchaveraged $C_{S K E}$ and (c) pitch-averaged energy loss coefficients at $1.4 C_{X}$

\subsubsection{Downstream Flow Field Measurements at 2.0 $C_{X}$}

Figure 9.7(a) and Figure 9.7(b) show the $C_{S K E}$ distributions further downstream at $2.0 C_{X}$. The $S K E$ dissipation between the two measurement planes brings about additional losses, in particular for SL2F: compared with SL2P, the secondary losses for SL2F occupy a larger portion of the flow field at $2.0 C_{X}$, and the distortion of the loss coefficient line contours have begun to affect the midspan flow. The results also indicate larger spanwise dissipation than pitchwise dissipation between the two planes; the secondary 
velocities have considerably smaller spanwise components as compared to those in Figure 9.6(a). In spite of the mixing, SL2F still shows considerable levels of $C_{S K E}^{\prime}$ at around $20 \%$ span, apparently due of the strong interaction between the passage vortex and the counter vortex. The lower values of SKE for SL2P indicate that there will be smaller mixing losses, which is ultimately a benefit in an engine.

(a)

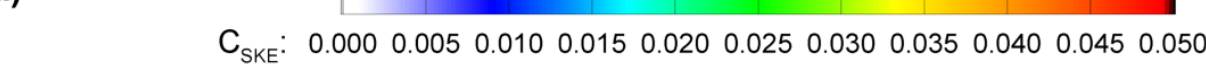
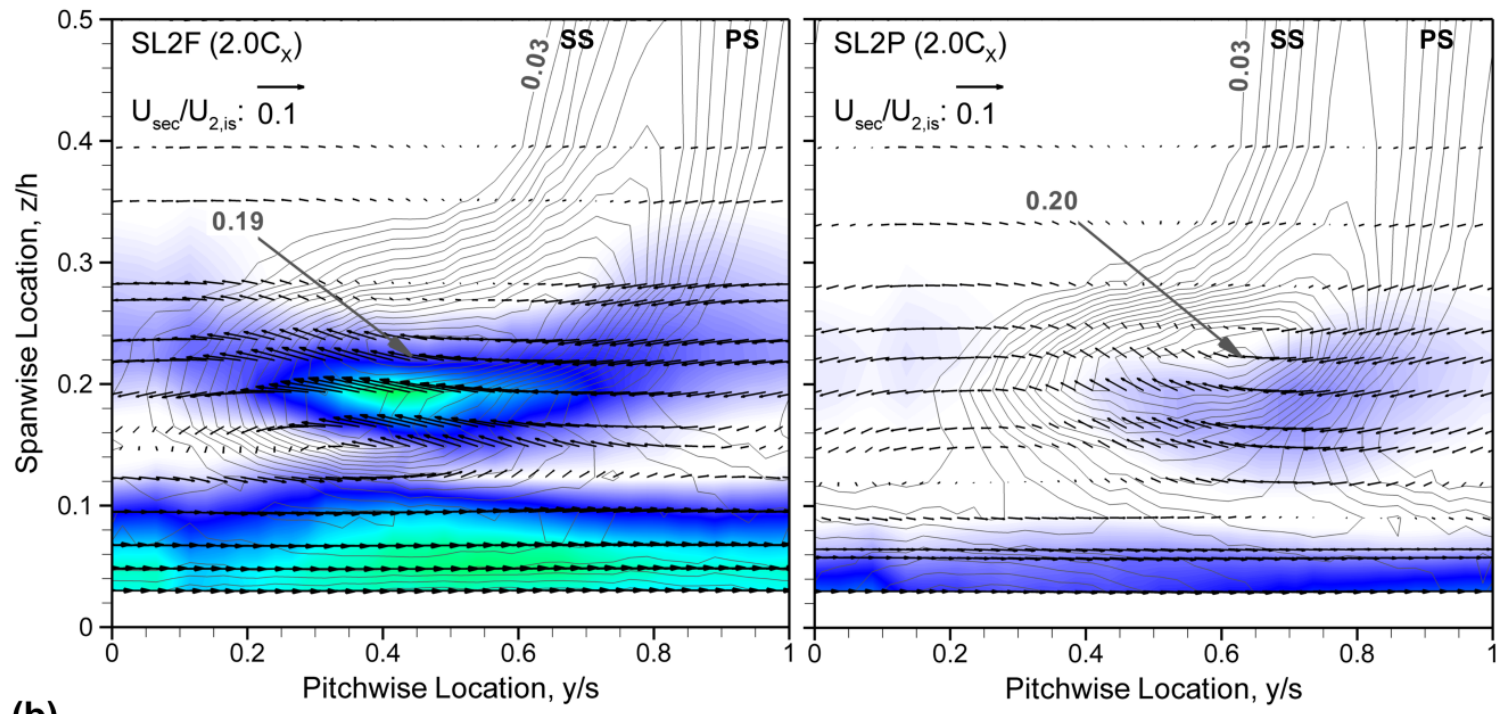

(b)

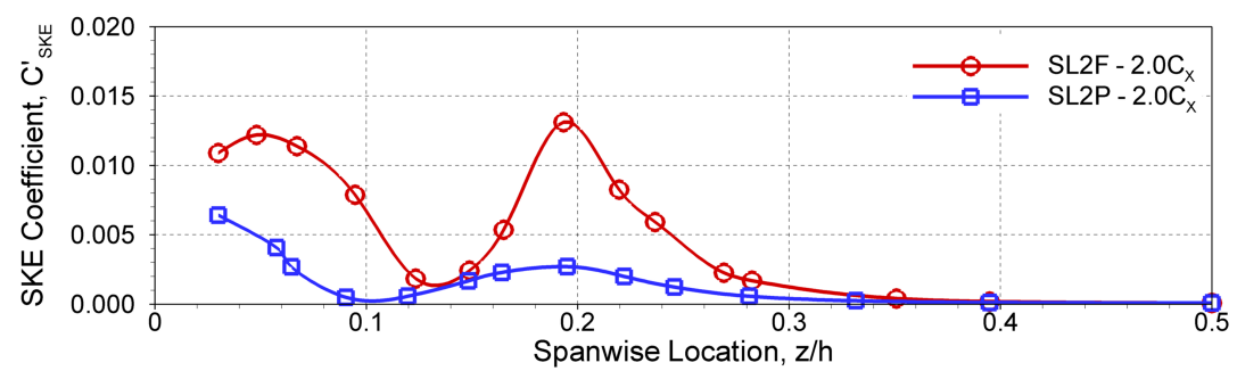

(c)

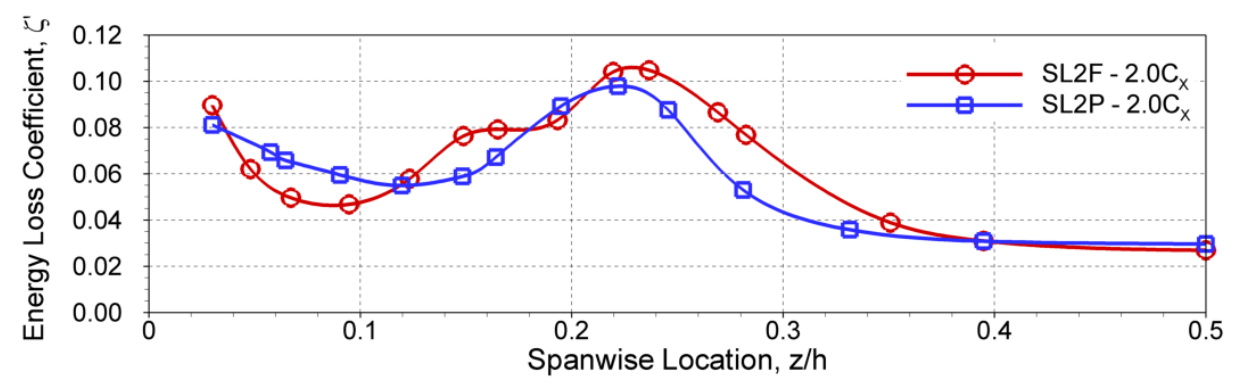

Figure 9.7 (a) Secondary kinetic energy coefficients and secondary velocities, (b) pitchaveraged $C_{S K E}$ and (c) pitch-averaged energy loss coefficients at $2.0 C_{X}$

The pitchwise mass-averaged energy loss coefficients at $2.0 C_{X}$ are shown in Figure 9.7(c). The contribution of near-wall losses increases substantially for SL2F, in part due 
to the increased mass fluxes, due to the more uniform mass flux distribution. SL2F produces larger losses than SL2P from about 12\% to 35\% span, and the midspan losses increase by about $10 \%$ for both cases. The pitch-averaged loss trends in the current data sets are similar to the results of Sauer et al. (2001), who investigated the effects of endwall leading edge bulbs on secondary flows; in their study, the secondary loss reduction was mainly attributed to the intensification of the suction-side leg of the horseshoe vortex, resulting in a weaker passage vortex.

\subsection{Computational Results at the Design Mach Number}

The CFD simulations were performed following the completion of the experiments, using the measured boundary conditions at the design outlet Mach number $\left(M_{2, i s}=0.78\right)$ and Reynolds number $\left(\operatorname{Re}_{C, 2}=600,000\right)$. The results from fully-turbulent simulations with the Shear Stress Transport turbulence model are presented in this section.

Figure 9.8 shows the predicted streamwise vorticity coefficients at $1.4 C_{X}$ for SL2F and SL2P. Also shown are the contour lines of mass-weighted energy loss coefficient. In comparison to the experimental results in Figure 9.5(a), the CFD predictions show stronger vortical structures and larger secondary losses.

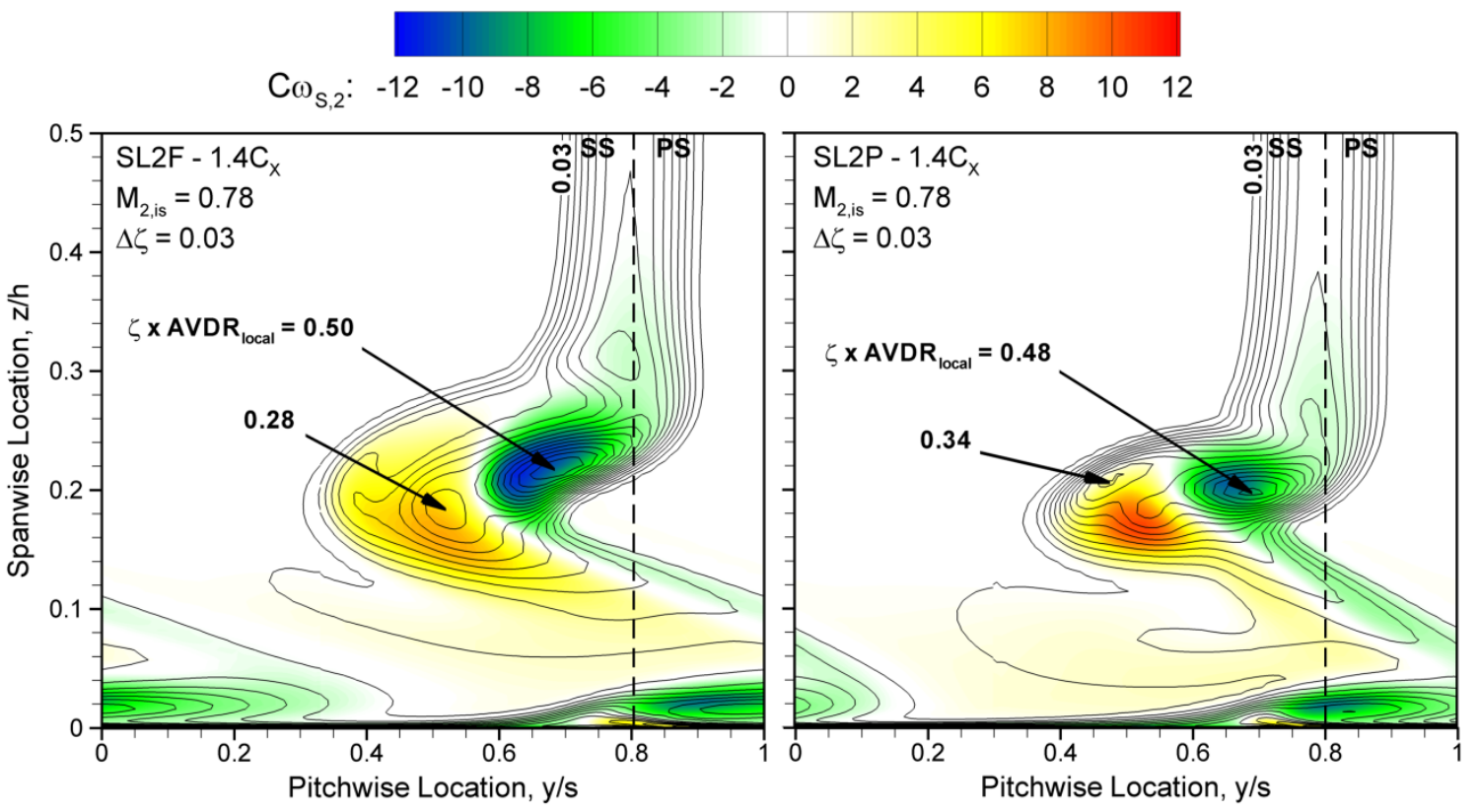

Figure 9.8 CFD predictions for SL2F and SL2P: colour floods of streamwise vorticity coefficient overlaid on contour lines of mass-weighted energy loss coefficient $\left(M_{2, i s}=0.78\right)$ 
As discussed in Chapter 6, SL2F and SL2P produce very similar profile losses in the computations. In Figure 9.8, SL2P shows lower secondary flow penetration than SL2F, as well as a significantly smaller and less intense counter vortex. The loss core coincident with the counter vortex is also smaller and has a lower peak magnitude for $\mathrm{SL} 2 \mathrm{P}(\zeta \times A V D R=0.48)$ than for SL2F $(0.50)$. The passage vortex, on the other hand, is considerably more intense for SL2P and the associated losses appear to be more concentrated, although they occupy a smaller portion of the flow field. Below 5\% span, both cascades have similar corner vortices and generate similar near-wall losses.

The pitchwise mass-averaged results from the experiments and the CFD are presented in Figure 9.9. The pitch-averaged exit flow angles show marked reductions in both underturning and overturning for SL2P. However, the numerical results overpredict the underturning by about $2^{\circ}$ for both cases. The location of maximum underturning in the computations is displaced slightly from $21 \%$ span for SL2F to $18 \%$ span for SL2P; this is consistent with the streamwise vorticity results in Figure 9.8(a). The numerical results are in better agreement with the measurements approaching the endwalls; as discussed in Chapter 8 , the computations seem to capture the overturning reasonably well. The experimental and computational results of Knezevici (2011) show very similar trends to those observed here, that CFD captures the overturning, but overpredicts the underturning.

CFD predicts a significant reduction of $C^{\prime}{ }_{S K E}$ for SL2P, as compared to SL2F. This is consistent with the reductions in the streamwise vorticity coefficients in Figure 9.8. The SKE predictions, however, are larger than the measured values for both cascades, particularly in the interaction region between the passage and counter vortices (at about $20 \%$ span). Similarly, the loss predictions are also higher than the measurements in this region. The $S K E$ and loss predictions are in better agreement with the measurements between $3 \%$ and $15 \%$ span, as well as in the $2 \mathrm{D}$ flow region.

The integrated numerical results indicate a 5\% mass-averaged row loss reduction for SL2P (as compared to SL2F) at the design Mach number. Therefore, the predicted massaveraged loss reduction for SL2P is smaller than the predicted benefit for endwall contouring, which was shown to be $10 \%$ for SL2C (as compared to SL2F) in Chapter 8. The experimental results are summarized in Section 9.5. 

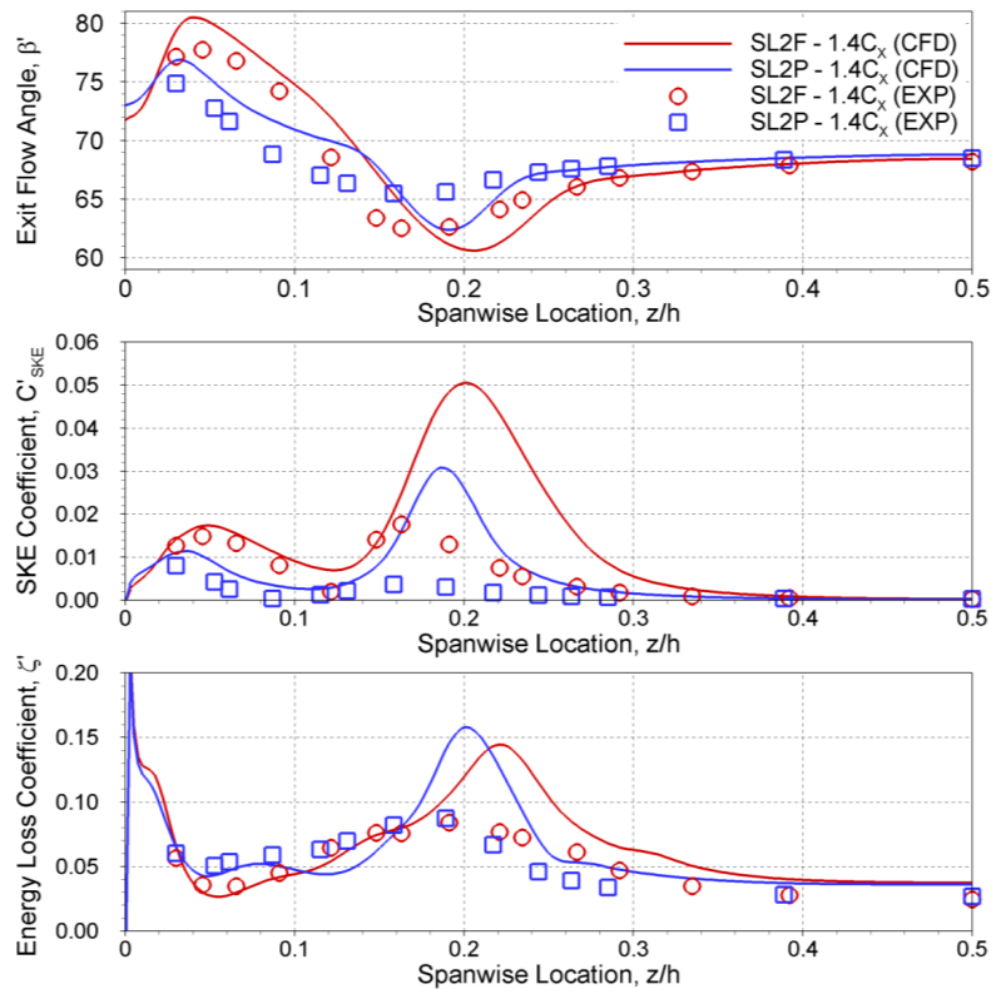

Figure 9.9 Experimental and numerical pitch-averaged results for SL2F and SL2P at 1.4C $C_{X}$ : exit flow angles, $S K E$ coefficients and energy loss coefficients $(M 2$,is $=0.78)$

\subsection{Integrated Results at both Design and Off-Design Mach Numbers}

The integrated results at the design Mach number are presented in Table 9.1. The results for SL2C (Chapter 8) are also included here for comparison purposes. SL2P generates smaller $C^{\prime \prime}$ SKE than SL2F at both measurement planes. At $1.4 C_{X}$, SL2P shows larger mass-averaged profile losses and smaller secondary losses than SL2F, resulting in slightly lower mass-averaged row losses. The experimental results, however, do not corroborate the predicted mass-averaged row loss reduction (-5\%) for SL2P at $1.4 C_{X}$ : the present CFD results overpredict the benefit.

At $2.0 C_{X}$, SL2P has generated smaller mass-averaged row losses than SL2F and SL2C. The mixed-out results from $2.0 C_{X}$ also indicate significant improvements for SL2P, as compared to SL2F: $15 \%$ reduction in mixed-out row losses and $38 \%$ reduction in secondary losses. On the other hand, SL2P produces about 10\% larger profile losses, as discussed in Chapter 6, and therefore it may be used for configurations that primarily benefit from endwall loss reduction, such as high-pressure turbines. Low-pressure turbines may exploit mix-loaded airfoils, comprising a profile similar to SL2P for the root 
and/or hub sections and a profile similar to SL2F for the profile flow region around the midspan.

Table 9.1 Overall integrated results for SL2F, SL2C and SL2P at the design Mach number

\begin{tabular}{|c|c|c|c|c|c|c|}
\hline & \multirow{2}{*}{\multicolumn{3}{|c|}{$1.4 C_{x}$}} & & & \\
\hline & & & & \multicolumn{3}{|c|}{$2.0 C_{X}$} \\
\hline & SL2F & SL2C & SL2P & SL2F & SL2C & SL2P \\
\hline \multicolumn{7}{|l|}{ Mass-Averaged } \\
\hline SKE Coefficient, C" SKE & 0.0056 & 0.0035 & 0.0017 & 0.0042 & 0.0027 & 0.0014 \\
\hline Energy Loss Coefficient, $\zeta$ & 0.036 & 0.036 & 0.035 & 0.048 & 0.045 & 0.044 \\
\hline Profile Energy Loss Coefficient, $\zeta_{P}$ & 0.024 & 0.024 & 0.026 & 0.026 & 0.025 & 0.029 \\
\hline Secondary Energy Loss Coefficient, $\zeta_{s}$ & 0.012 & 0.012 & 0.009 & 0.022 & 0.020 & 0.015 \\
\hline \multicolumn{7}{|l|}{ Mixed-Out } \\
\hline Energy Loss Coefficient, $\zeta$ & 0.045 & 0.042 & 0.040 & 0.056 & 0.051 & 0.048 \\
\hline Profile Energy Loss Coefficient, $\zeta_{P}$ & 0.025 & 0.025 & 0.028 & 0.026 & 0.026 & 0.029 \\
\hline Secondary Energy Loss Coefficient, $\zeta_{s}$ & 0.020 & 0.017 & 0.012 & 0.030 & 0.025 & 0.019 \\
\hline
\end{tabular}

The integrated results for three different exit Mach numbers are presented in Table 9.2. As discussed in Chapter 6, raising the Mach number is accompanied with increased aft-loading, higher Reynolds numbers and higher row velocity ratios. In Table 9.2, the loss coefficients and $C^{\prime \prime}{ }_{S K E}$ both decrease with Mach number. SL2P shows a larger benefit in terms of the mixed-out loss coefficient than in terms of the mass-averaged loss coefficient at all investigated Mach numbers. The measured benefit for SL2P, however, diminishes at the highest Mach number partly due to the lower secondary loss production for both cascades (perhaps due to increased aft loading). The results for the contoured cascades, SL1C and SL2C, indicated a similar decrease in endwall contouring benefit at higher Mach numbers in Chapter 8.

Table 9.2 Integrated results for SL2F and SL2P at three different Mach numbers $\left(1.4 C_{X}\right)$

\begin{tabular}{|c|c|c|c|c|c|c|}
\cline { 2 - 7 } \multicolumn{1}{c|}{} & SL2F & SL2P & SL2F & SL2P & SL2F & SL2P \\
\hline Outlet Isentropic Mach Number, $\boldsymbol{M}_{2, \text { is }}$ & 0.65 & 0.65 & 0.78 & 0.78 & 0.91 & 0.90 \\
\hline Zweifel Coefficient, $\mathbf{Z} \boldsymbol{w}$ & 1.07 & 1.07 & 0.97 & 0.99 & 0.89 & 0.90 \\
\hline Mass-Ave. SKE Coefficient, $\boldsymbol{C}^{\prime \prime}$ sKE & 0.0065 & 0.0021 & 0.0056 & 0.0017 & 0.0050 & 0.0016 \\
\hline Mass-Ave. Row Loss Coefficient, $\boldsymbol{\zeta}$ & 0.038 & 0.037 & 0.036 & 0.035 & 0.033 & 0.033 \\
\hline Mixed-Out Row Loss Coefficient, $\boldsymbol{\zeta}$ & 0.048 & 0.043 & 0.045 & 0.040 & 0.041 & 0.039 \\
\hline
\end{tabular}


The mixed-out loss coefficients for SL2P at three different axial locations are presented graphically in Figure 9.10 (the results form additional traverse plane at $1.7 C_{X}$ are included here). As previously noted, both profile and secondary losses decrease with Mach number. Profile losses are markedly larger than secondary losses at $1.4 C_{X}$ possibly related to the high airfoil aspect ratios. Secondary losses increase with downstream distance: a $25 \%$ increase at $1.7 C_{X}$ and $50 \%$ increase at $2.0 C_{X}$ at the design Mach number. The largest row loss coefficient in Figure 9.10 corresponds to the lowest Mach number $\left(M_{2, i s}=0.65\right)$ at $2.0 C_{X}$.

Figure 9.10 Mixed-out loss coefficient variation with Mach number from three different axial locations for SL2P

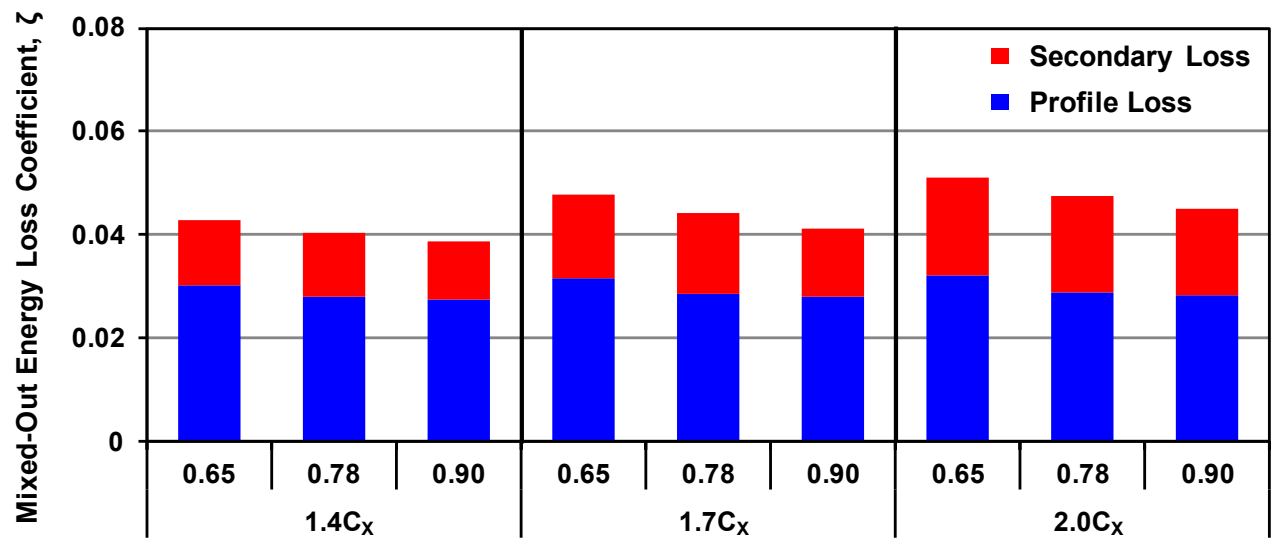




\section{Chapter 10}

\section{Summary, Conclusions and Recommendations}

\subsection{Summary}

The aerodynamic performance of a family of linear turbine cascades, designed and provided by PWA, were examined with the goal of improving the understanding of the secondary flow mechanisms in the transonic flow regime. The turbine airfoils were designated SL for "Secondary Loss", and grouped by both total flow turning and overall loading level to develop a comprehensive dataset:

- low flow turning $\left(\theta=90^{\circ}\right)$ : SL3 and SL4

- $\quad$ high flow turning $\left(\theta=112^{\circ}\right)$ : SL1, SL2 and SL2P

- $\quad$ baseline loaded $(\mathrm{Zw} \sim 0.75):$ SL1 and SL3

- $\quad$ more highly loaded (Zw 1.0): SL2, SL2P and SL4

The investigations were primarily based on detailed experimental measurements at the High-Speed Wind Tunnel Laboratory at Carleton University; these included both pressure probe measurements and surface flow visualization studies at design and offdesign Mach numbers. In addition, numerical investigations provided complementary results, which were not directly available from the experiments.

An important objective in modern gas turbine design is to reduce the cost of the engine. The cost reduction, however, should not have a negative impact on the aerodynamic performance or efficiency of the engine. In the current project, the cost reduction is associated with reducing the number of turbine blades within the highpressure stages, which results in both fewer turbine blades and lower engine weight. To extract the same work from a turbine stage with fewer blades, each blade is subjected to larger aerodynamic loadings that may result in higher losses and lower efficiency. Therefore, any new strategy for cost reduction is typically validated through a series of 
technology readiness levels (TRL), which were described in Chapter 2. The current research project is identified as TRL-4: second stage of experimental testing.

A summary of the available literature on the subject was provided in Chapter 2, which points to the scarcity of secondary flow data from high-speed linear turbine cascades. The results from the current work aim to bridge the gap between low-speed linear cascade testing and high-speed rotating test rigs. The experimental apparatus and measurement procedures were described in Chapter 3. As a prerequisite to testing, the test section flow quality was also documented to demonstrate acceptable inlet flow uniformity and outlet flow periodicity. The data reduction procedures and uncertainty analysis were discussed in Chapter 4, and the computational procedures were described in Chapter 5. In particular, the impact of inlet turbulence intensity and length scale on the numerical predictions were investigated to assess their contributions to the overall numerical uncertainty.

The experimental and numerical results were presented in Chapters 6 through 9. As a first step, the profile flow measurements were examined and compared to the CFD predictions in Chapter 6 to identify any potential discrepancies from design intent. The secondary flow results from cascades with flat endwalls were presented in Chapter 7. The effects of varying the exit Mach number on secondary flows and losses were also examined here. The application of endwall contouring to reduce the secondary losses was investigated in Chapter 8. The baseline-loaded, SL1, and the more-highly-loaded, SL2, configurations were selected for this study to assess the effects of the overall loading level on endwall contouring effectiveness. The influence of airfoil pressure-side modification on secondary flows was documented based on the results from SL2 and SL2P cascades in Chapter 9.

\subsection{Conclusions}

An improved understanding of the loss generating mechanisms in transonic turbines has been developed through detailed experimental and computational investigations. The main conclusions and contributions of the thesis are summarized here.

The midspan aerodynamic performance of the turbine cascades were examined in terms of the airfoil loading distributions and the profile losses. The more highly-loaded 
cascades, SL2, SL2P and SL4, display larger suction-surface Mach numbers and larger adverse pressure gradients past the suction peak than SL1 and SL3. The flow visualization results also suggest evidence of a small region of flow separation on the suction sides of SL1, SL2 and SL2P. Consequently, SL2P generates the highest profile losses in the experiments. Increasing the outlet Mach number results in larger flow acceleration through the passage and more aft-loaded blade loading distributions. The profile energy loss coefficients, however, do not vary significantly for the operating points examined here $\left(0.65<\mathrm{M}_{\text {exit }}<0.91\right)$.

The secondary flow results from cascades with flat endwalls were presented in two separate sections: low flow turning and high flow turning cascades. Overall, the highturning cascades produce higher row losses than the low-turning cascades. The highturning cascades also exhibit generally stronger vortical structures and higher secondary flow penetration depth than the low-turning cascades, as indicated by the spanwise extents of the vortical structures.

Based on the results from the low-turning cascades, the more highly-loaded SL4F displays stronger vortical structures and generates higher secondary losses than SL3F. In particular, a more intense passage vortex results in larger flow overturning and higher secondary kinetic energy production for SL4F. In contrast to SL4F, the secondary flow structures for SL3F are located closer to the endwall and there is no evidence of a corner vortex, perhaps due to its close proximity to the endwall. Overall, SL4F generates $7 \%$ higher mixed-out row losses than SL3F as measured by the energy loss coefficient.

The results from the high-turning cascades comprised probe measurements, surface flow visualization pictures and CFD predictions. The probe measurements at $1.4 C_{X}$ indicate similar row loss coefficients for the two cascades despite the $30 \%$ higher Zweifel coefficient for SL2F. The endwall flow visualization results indicate higher endwall shear stresses for SL1F than SL2F, particularly in the forward part of the passage and near the pressure surface. On the other hand, the SL2F results indicate higher secondary flow penetration depth at the trailing edge. SL2F generates stronger vortical structures, larger exit flow angle variations, larger secondary velocities and higher secondary kinetic energy at $1.4 C_{X}$. Additional probe traverses at $2.0 C_{X}$ show significant differences in the 
secondary flow fields: SL2F ultimately produces $23 \%$ higher mixed-out row losses than SL1F in part due to the mixing out and dissipation of the larger secondary kinetic energy.

Raising the outlet Mach number results in smaller streamwise vorticity coefficients associated with the passage and the counter vortices. This is accompanied with spanwise displacement of the vortices towards the endwall, smaller flow underturning and overturning, and smaller secondary velocities. Accordingly, the secondary kinetic energy and the secondary losses decrease as the outlet Mach number is raised in the subsonic regime.

Non-axisymmetric endwall contouring was applied to the SL1 and SL2 configurations based on the method of Praisner et al. (2007), modified for compressible CFD calculations. The contoured endwall geometries were optimized with the goal of reducing the secondary losses. The application of endwall contouring leads to weaker interactions between the vortical structures and the suction-surface boundary layers, and more favourable, streamwise-oriented, mid-passage crossflows based on the surface flow visualization results, at the expense of increased wetted areas.

The initial probe measurements at $1.4 C_{X}$, however, do not confirm the numerically predicted row loss reductions. The aerodynamic performance of the baseline-loaded cascades, SL1F and SL1C, shows negligible changes due to contouring. The more highly-loaded SL2C cascade, on the other hand, produces smaller and less intense vortical structures and lower secondary kinetic energy than SL2F. This is accompanied with reduced under/overturning from $5 \%$ to $50 \%$ span, qualitatively validating the CFD predictions. The second set of measurements at $2.0 C_{X}$ display lower mass-averaged row losses for SL2C, associated with smaller mixing losses between the two planes. Consequently, the fully mixed-out results indicate an $8 \%$ row loss reduction for SL2C at the design Mach number as compared to SL2F.

Operating at off-design Mach numbers also influences the results: reducing the Mach number enhances the effectiveness of endwall contouring, whereas increasing the Mach number reduces the benefit due to increased aft loading. The results suggest that the application of endwall contouring is more appropriate for front- or mid-loaded airfoils. Overall, the results confirm the low-speed findings in cascades with similar endwall 
designs (Knezevici, 2011) that contouring is a viable method to reduce the secondary losses in transonic turbines.

A novel approach for endwall loss reduction in transonic turbines was also investigated here. The new technique aims to modify the $2 \mathrm{D}$ blade profile in an effort to raise the fluid momentum near the pressure surface of the airfoil. The pressure-side modification may be used either in place of or combined with non-axisymmetric endwall contouring. The new airfoil, which is based on the SL2 geometry, is designated SL2P.

The CFD predictions indicate weaker endwall crossflows, reduced secondary flow penetration depth and a 5\% reduction in mass-averaged row losses for SL2P. The surface flow visualization results confirm the CFD predictions particularly regarding the weaker interaction between the passage vortex and the suction-side boundary layer. The downstream measurements also exhibit significant reductions in both passage vortex and counter vortex intensities, resulting in reduced under/overturning for SL2P; accordingly, SL2P generates $70 \%$ lower secondary kinetic energy than SL2F at $1.4 C_{X}$. The measurements at $2.0 C_{X}$ demonstrate additional benefits for SL2P in terms of loss reduction due to higher primary and secondary kinetic energy dissipation for SL2F. In comparison to the measured losses for SL2F, the fully mixed-out results indicate a $38 \%$ reduction in secondary losses for SL2P, which exceeds the 15\% reduction for SL2C. On the other hand, SL2P generates approximately 10\% larger profile losses than SL2F and SL2C. Therefore, SL2P is deemed a suitable design-point alternative to endwall contouring for low aspect ratio applications, such as high-pressure turbines. Operating at higher off-design Mach numbers reduces the benefit in terms of the mixed-out row losses due to increased airfoil aft loading.

\subsection{Recommendations for Future Work}

Additional research areas were identified during the course of the experimental investigations. Even though the results from the current project form a large dataset, experimental data from additional cascades would be required to assess the impact of front- and mid-loading distributions on the secondary flows. Front loading is typically found to reduce the profile losses in low-speed cascades, whereas aft-loading reduces the 
secondary losses. Therefore, the performance of mixed-loaded transonic airfoils (e.g. front loaded at midspan and aft loaded near the endwall) should also be investigated.

In an aero engine, the range of incidence variations seen by an HPT tends to be small. Nonetheless, as a design requirement, the profile performance of a new turbine airfoil is typically assessed for a range of inlet incidences associated with off-design operation. As shown, operating at off-design Mach numbers alters the airfoil loading distributions and the secondary losses. Operating at off-design incidence is expected to alter the secondary flow structures in a similar fashion. Therefore, the effects of inlet incidence on secondary losses in the transonic flow regime should also be investigated. The results from these studies may be used to improve the secondary loss correlations.

As discussed in Chapter 2, the turbulence measurements from low-speed linear turbine cascades have provided valuable insights in to the secondary loss generating mechanisms. The use of hot-wire probes in transonic cascades is more challenging due to the high Mach numbers and the temperature drops associated with air expansion. Therefore, optical techniques such as Laser Doppler Anemometry (LDA) and Particle Image Velocimetry (PIV) may be used instead to document the turbulence kinetic energy production and dissipation within and downstream of the cascades. The frequency spectrum of turbulence may be used to examine any potential unsteadiness in the secondary flow field.

Although the application of endwall contouring is demonstrated to positively influence the aerodynamic performance of the cascades, secondary loss predictions in high-speed flows and particularly the contributions from secondary kinetic energy dissipation require further analysis. The discrepancies between the numerical predictions and the measurements also highlight the need for further experimental studies. Additional experimental studies at off-design incidence are paramount to assess the risk associated with endwall contouring.

A recent study by Gregory-Smith et al. (2008) from a low-speed facility showed similar benefits for "conservative" endwall profiling and leading edge extensions that were attributed to the unloading of the leading edge; combining the two mechanisms resulted in a $20 \%$ row loss reduction compared to the baseline case. Therefore, the 
effects of combining the pressure-side modification with endwall contouring in highspeed cascades also require further analysis.

The results presented here correspond to a highly-simplified, linear cascade test configuration. This is essential to isolate and study the effects of flow compressibility on secondary flows and secondary losses. Additional features such as inlet and outlet cavities, and cooling and leakage flows may also be simulated in the linear cascade configuration. The effects of radial pressure gradients, skewed inlet boundary layers and upstream wakes on secondary flows are typically investigated at TRL-5: high-speed rotating test rigs.

Finally, the comparisons between the experimental data and the CFD predictions highlight the need for additional numerical studies. In particular, it was evident that the CFD results do not capture the diffusion and dissipation of the secondary flow structures accurately. Therefore, the current experimental results should be utilized to validate any potential improvements in CFD turbulence modeling in the future. 


\section{References}

Adjlout, L., and Dixon, S. L. (1992), "Endwall Losses and Flow Unsteadiness in a Turbine Blade Cascade,” ASME J. Turbomach., 114, 191-197.

Amecke, J., and Safarik, P. (1995), "Data Reduction of Wake Flow Measurements with Injection of an Other Gas," Forschungsbericht DLR-FB 95-32, Cologne, Germany.

Aunapu, N. V., Volino, R. J., Flack, K. A., and Stoddard, R. M. (2000), "Secondary Flow Measurements in a Turbine Passage With Endwall Flow Modification," ASME J. Turbomach., 122, 651-658.

Bagshaw, D. A., Ingram, G. L., Gregory-Smith, D. G., and Stokes, M. R. (2008), "An experimental study of three-dimensional turbine blades combined with profiled endwalls," Proc. Instn. Mech. Engrs., Part A: J. Power and Energy, 222, 103-110.

Bario, F., Leboeuf, F., and Papailiou, K. D. (1982), "Study of Secondary Flows in Blade Cascades of Turbomachines,” ASME J. Eng. Power, 104, 497-509.

Bassi, F., Osnaghi, C., Perdichizzi, A., and Savini, M. (1989), "Secondary Flows in a Transonic Cascade: Comparison between Experimental and Numerical Results,” ASME J. Fluid Eng., 111, 369-377.

Bassi, F., and Perdichizzi, A. (1987), "Secondary Flow Development Downstream of a Transonic Cascade," Proc. of The International Gas Turbine Congress (87-Tokyo-IGTC-17), pp. 123130, Tokyo, Japan.

Becz, S., Majewski, M. S., and Langston, L. S. (2003), "Leading Edge Modification Effects on Turbine Cascade Endwall Loss," Proc. of Turbo Expo 2003 (GT2003-38898), Atlanta, GA, USA.

Benner, M. W. (2003), "The Influence of Leading-Edge Geometry on Profile and Secondary Losses in Turbine Cascades," Ph.D. thesis, Carleton University, Ottawa, Canada. 
Benner, M. W., Sjolander, S. A., and Moustapha, S. H. (1997), "Influence of Leading-Edge Geometry on Profile Losses in Turbines at Off-Design Incidence: Experimental Results and an Improved Correlation,” ASME J. Turbomach., 119, 193-200.

Benner, M. W., Sjolander, S. A., and Moustapha, S. H. (2004), “The Influence of Leading-Edge Geometry on Secondary Losses in a Turbine Cascade at the Design Incidence," ASME J. Turbomach., 126, 277-287.

Benner, M. W., Sjolander, S. A., and Moustapha, S. H. (2006a), “An Empirical Prediction Method for Secondary Losses in Turbines - Part I: A New Loss Breakdown Scheme and Penetration Depth Correlation,” ASME J. Turbomach., 128, 273-280.

Benner, M. W., Sjolander, S. A., and Moustapha, S. H. (2006b), “An Empirical Prediction Method for Secondary Losses in Turbines - Part II: A New Secondary Loss Correlation," ASME J. Turbomach., 128, 281-291.

Binder, A., and Romey, R. (1983), "Secondary Flow Effects and Mixing of the Wake Behind a Turbine Stator,” ASME J. Eng. Power, 105, 40-46.

Bradshaw, P. (1996), "Turbulence Modeling with Application to Turbomachinery," Progress in Aerospace Sciences, 32, 575-624.

Brear, M. J., Hodson, H. P., Gonzalez, P., and Harvey, N. W. (2002), "Pressure Surface Separations in Low-Pressure Turbines-Part 2: Interactions with the Secondary Flow," ASME J. Turbomach., 124, 402-409.

Brennan, G., Harvey, N. W., Rose, M. G., Fomilson, N., and Taylor, M. D. (2003), “Improving the Efficiency of the Trent 500-HP Turbine Using Nonaxisymmetric End Walls - Part I: Turbine Design,” ASME J. Turbomach., 125, 497-504.

Brown, L. E. (1972), “Axial Flow Compressor and Turbine Loss Coefficients: A Comparison of Several Parameters,” ASME J. Eng. Power, 94 Ser A, 193-201.

Came, P. M. (1973), “Secondary Loss Measurements in a Cascade of Turbine Blades," Proc. of Conference on Heat and Fluid Flow in Steam and Gas Turbine Plant (Conference Publication 3), pp. 75-83, Coventry, England, UK.

Came, P. M., and Marsh, H. (1974), "Secondary Flow in Cascades: Two Simple Derivations for the Components of Vorticity," J. Mechanical Engineering Science, 16, 391-401. 
Camus, J. J., Denton, J. D., Scrivener, C. T. J., and Soulis, J. V. (1984), “An Experimental and Computational Study of Transonic Three-Dimensional Flow in a Turbine Cascade," ASME J. Eng. Gas Turbines Power, 106, 414-420.

Chen, L. D., and Dixon, S. L. (1986), "Growth of Secondary Flow Losses Downstream of a Turbine Blade Cascade,” ASME J. Eng. Gas Turbines Power, 108, 270-276.

Chue, S. H. (1975), "Pressure Probes for Fluid Measurement," Progress in Aerospace Sciences, 16, 147-223.

Chung, J. T., Simon, T. W., and Buddhavarapu, J. (1991), “Three-Dimensional Flow Near the Blade/Endwall Junction of a Gas Turbine: Application of a Boundary Layer Fence," Proc. of International Gas Turbine and Aeroengine Congress and Exposition (91-GT-45), Orlando, FL, USA.

Chung, J. T., and Simon, T. W. (1993), "Effectiveness of the Gas Turbine Endwall Fences in Secondary Flow Control at Elevated Freestream Turbulence Levels," Proc. of International Gas Turbine and Aeroengine Congress and Exposition (93-GT-51), Cincinnati, OH, USA.

Contini, D., Manfrida, G., Michelassi, V., and Riccio, G. (2000), "Measurements of Vortex Shedding and Wake Decay Downstream of a Turbine Inlet Guide Vane," Flow, Turbulence and Combustion, 64, 253-278.

Corriveau, D. (2005), "Influence of Loading Distribution on the Performance of High Pressure Turbine Blades," Ph.D. thesis, Carleton University, Ottawa, Canada.

Corriveau, D., and Sjolander, S. A. (2002), "Impact of Flow Quality in Transonic Cascade Wind Tunnels- Measurements in an HP Turbine Cascade," Proc. of ICAS 2002 Congress (511R1), Toronto, Canada.

Corriveau, D., and Sjolander, S. A. (2004a), "Experimental and Numerical Investigation on the Performance of a Family of Three HP Transonic Turbine Blades," Proc. of Turbo Expo 2004 (GT2004-53087), Vienna, Austria.

Corriveau, D., and Sjolander, S. A. (2004b), "Influence of Loading Distribution on the Performance of Transonic High Pressure Turbine Blades," ASME J. Turbomach., 126, 288296.

Corriveau, D., and Sjolander, S. A. (2005), “Aerodynamic Performance of a Family of Three High Pressure Transonic Turbine Blades at Off-Design Incidence," Proc. of Turbo Expo 2005 (GT2005-68159), Reno-Tahoe, NV, USA. 
Corriveau, D., and Sjolander, S. A. (2007), "Influence of Loading Distribution on the Off-Design Performance of High-Pressure Turbine Blades," ASME J. Turbomach., 129, 563-571.

Cumpsty, N. A., and Horlock, J. H. (2006), “Averaging Nonuniform Flow for a Purpose,” ASME J. Turbomach., 128, 120-129.

Davidson, P. A. (2005), “Turbulence: An Introduction for Scientists and Engineers,” Book, pp. 39-48, Oxford, UK: Oxford University Press.

de la Rosa Blanco, E., Hodson, H. P., Vasquez, R., and Torre, D. (2003), "Influence of the State of the Inlet Endwall Boundary Layer on the Interaction Between Pressure Surface Separation and Endwall Flows. Part A: J. Power and Energy,” 217, 433-441.

Denton, J. D. (1993), “The 1993 IGTI Scholar Lecture: Loss Mechanisms in Turbomachines,” ASME J. Turbomach., 115, 621-656.

Denton, J. D., and Xu, L. (1990), “The Trailing Edge Loss of Transonic Turbine Blades,” ASME J. Turbomach., 112, 277-285.

Detemple-Laake, E. (1990), "Measurements of the Flow Field in the Blade Passage and SideWall Region of a Plane Turbine Cascde," AGARD-CP-469: Secondary Flows in Turbomachines, Paper No. 10.

Dominy, R. G., and Harding, S. C. (1990), “An Investigation of Secondary Flows in Nozzle Guide Vanes," AGARD-CP-469: Secondary Flows in Turbomachines, Paper No. 7.

Dominy, R. G., and Hodson, H. P. (1993), "An Investigation of Factors Influencing the Calibration of Five-Hole Probes for Three-Dimensional Flow Measurements," ASME J. Turbomach., 115, 513-519.

Dossena, V., D'Ippolito, G., and Pesatori, E. (2004), "Stagger Angle and Pitch-Chord Ratio Effects on Secondary Flows Downstream of a Turbine Cascade at Several Off-Design Conditions," Proc. of Turbo Expo 2004 (GT2004-54083), Vienna, Austria.

Dossena, V., Perdichizzi, A., and Savini, M. (1999), “The Influence of Endwall Contouring on the Performance of a Turbine Nozzle Guide Vane,” ASME J. Turbomach., 121, 200-208.

Duden, A., and Fottner, L. (1997), "Influence of Taper, Reynolds Number and Mach Number on the Secondary Flow Field of a Highly Loaded Turbine Cascade," Part A: J. Power and Energy, 211, 309-320. 
Duden, A., Raab, I., and Fottner, L. (1999), "Controlling the Secondary Flow in a Turbine Cascade by Three-Dimensional Airfoil Design and Endwall Contouring," ASME J. Turbomach., 121, 191-199.

Dunham, J. (1970), “A Review of Cascade Data on Secondary Losses in Turbines," J. Mechanical Engineering Science, 12, 48-59.

Eckerle, W. A., and Langston, L. S. (1987), "Horseshoe Vortex Formation Around a Cylinder," ASME J. Turbomach., 109, 278-285.

Germain, T., Nagel, M., Raab, I., Schupbach, P., Abhari, R. S., and Rose, M. (2010), “Improving Efficiency of a High Work Turbine Using Nonaxisymmetric Endwalls - Part I: Endwall Design and Performance,” ASME J. Turbomach., 132, 021007.

Gregory-Smith, D. G., Graves, C. P., and Walsh, J. A. (1988a), "Growth of Secondary Losses and Vorticity in an Axial Turbine Cascade," ASME J. Turbomach., 110, 1-8.

Gregory-Smith, D. G., Walsh, J. A., Graves, C. P., and Fulton, K. P. (1988b), “Turbulence Measurements and Secondary Flows in a Turbine Rotor Cascade," ASME J. Turbomach., 110, 479-485.

Gregory-Smith, D. G., and Cleak, J. G. E. (1992), "Secondary Flow Measurements in a Turbine Cascade With High Inlet Turbulence,” ASME J. Turbomach., 114, 173-183.

Gregory-Smith, D. G. (1997), "Secondary and Tip-Clearance Flows in Axial Turbines,” Lecture Series 1997-01, Rhode St. Genese, Belgium: Von Karman Institute for Fluid Dynamics.

Gregory-Smith, D. G., Ingram, G., Jayaraman, P., Harvey, N. W., and Rose, M. G. (2001), "Nonaxisymmetric turbine end wall profiling," Proc. Instn. Mech. Engrs., Part A: J. Power and Energy, 215, 721-734.

Gregory-Smith, D., Bagshaw, D., Ingram, G., and Stokes, M. (2008), "Using Profiled Endwalls, Blade Lean and Leading Edge Extensions to Minimize Secondary Flow," Proc. of Turbo Expo 2008 (GT2008-50811), Berlin, Germany.

Gustafson, R., Mahmood, G., and Acharya, S. (2007), “Aerodynamic Measurements in a Linear Turbine Blade Passage With Three-Dimensional Endwall Contouring," Proc. of Turbo Expo 2007 (GT2007-28073), Montreal, Canada.

Harrison, S. (1990), "Secondary Loss Generation in a Linear Cascade of High-Turning Turbine Blades,” ASME J. Turbomach., 112, 618-624. 
Harrison, S. (1992), "The Influence of Blade Lean on Turbine Losses," ASME J. Turbomach., 114, 184-190.

Hartland, J. C., Gregory-Smith, D. G., Harvey, N. W., and Rose, M. G. (2000), “Nonaxisymmetric Turbine End Wall Design: Part II - Experimental Validation," ASME J. Turbomach., 122, 286-293.

Hartland, J., and Gregory-Smith, D. (2002), “A Design Method for the Profiling of End Walls in Turbines," Proc. of Turbo Expo 2002 (GT-2002-30433), Amsterdam, The Netherlands.

Harvey, N. W., Rose, M. G., Taylor, M. D., Shahpar, S., Hartland, J., and Gregory-Smith, D. G. (2000), "Nonaxisymmetric Turbine End Wall Design: Part I - Three-Dimensional Linear Design System,” ASME J. Turbomach., 122, 278-285.

Harvey, N. W., Brennan, G., Newman, D. A., and Rose, M. G. (2002), "Improving Turbine Efficiency Using Non-Axisymmetric End Walls: Validation in the Multi-Row Environment and With Low Aspect Ratio Blading," Proc. of Turbo Expo 2002 (GT-2002-30337), Amsterdam, The Netherlands.

Hjärne, J., Chernoray, V., Larsson, J., and Lofdahl, L. (2007), "Numerical Validations of Secondary Flows and Loss Development Downstream of a Highly Loaded Low Pressure Turbine Outlet Guide Vane Cascade,” Proc. of Turbo Expo 2007 (GT2007-27712), Montreal, Canada.

Hodson, H. P., and Dominy, R. G. (1987), “Three-Dimensional Flow in a Low-Pressure Turbine Cascade at Its Design Condition,” ASME J. Turbomach., 109, 177-185.

Hoheisel, H., Kiock, R., Lichtfuss, H. J., and Fottner, L. (1987), "Influence of Free-Stream Turbulence and Blade Pressure Gradient on Boundary Layer and Loss Behavior of Turbine Cascades,” ASME J. Turbomach., 109, 210-219.

Hourmouziadis, J. (1989), “Aerodynamic Design of Low Pressure Turbines,” AGARD-LS-167: Blading Design for Axial Turbomachines, Paper No. 8.

Ingram, G., Gregory-Smith, D., Rose, M., Harvey, N., and Brennan, G. (2002), “The Effect of End-Wall Profiling on Secondary Flow and Loss Development in a Turbine Cascade," Proc. of Turbo Expo 2002 (GT-2002-30339), Amsterdam, The Netherlands.

Ingram, G. L. (2003), "Endwall Profiling for the Reduction of Secondary Flow in Turbines," Ph.D. thesis, University of Durham, Durham, England, UK. 
Ingram, G., Gregory-Smith, D., and Harvey, N. (2005a), "The Benefits of Turbine Endwall Profiling in a Cascade,” Proc. Instn. Mech. Engrs., Part A: J. Power and Energy, 219, 49-59. Ingram, G., Gregory-Smith, D., and Harvey, N. (2005b), "Investigation of a Novel Secondary Flow Feature in a Turbine Cascade with End Wall Profiling," ASME J. Turbomach., 127, 209-214.

Islam, A. M. T. (1999), "An Experimental and Computational Study of the Aerodynamics of Turbine Blades with Damage,” Ph.D. thesis, Carleton University, Ottawa, Canada.

Jeffries, M. S. (1994), "The Commissioning and Enhancement of a Blow Down Wind Tunnel," Master's thesis, Carleton University, Ottawa, Canada.

Jeffries, M. S., Jouini, D. B. M., and Sjolander, S. A. (1997), "Determining the Sampling Rates and Times in a High Speed Wind Tunnel," Proc. of CASI 6th Symposium on Aerodynamics, Toronto, Canada.

Jeffries, M. S. (2000), "Initial Investigations of Transonic Turbine Aerodynamics Using the Carleton University High-Speed Wind Tunnel," Ph.D. thesis, Carleton University, Ottawa, Canada.

Johansen, E. S., Rediniotis, O. K., and Jones, G. (2001), "The Compressible Calibration of Miniature Multi-Hole Probes,” ASME J. Fluid Eng., 123, 128-138.

Jouini, D. B. M. (2000), “Experimental Investigation of Two Transonic Linear Turbine Cascades at Off-Design Conditions," Ph.D. thesis, Carleton University, Ottawa, Canada.

Kacker, S. C., and Okapuu, U. (1982), “A Mean Line Prediction Method for Axial Flow Turbine Efficiency,” ASME J. Eng. Power, 104, 111-119.

Knezevici, D. C., Sjolander, S. A., Praisner, T. J., Allen-Bradley, E., and Grover, E. A. (2009), "Measurements of Secondary Losses in a High-Lift Front-Loaded Turbine Cascade With the Implementation of Non-Axisymmetric Endwall Contouring," Proc. of Turbo Expo 2009 (GT2009-59677), Orlando, FL, USA.

Knezevici, D. C., Sjolander, S. A., Praisner, T. J., Allen-Bradley, E., and Grover, E. A. (2010), "Measurements of Secondary Losses in a Turbine Cascade With the Implementation of Nonaxisymmetric Endwall Contouring,” ASME J. Turbomach., 132, 011013.

Knezevici, D. C. (2011), "Controlling Secondary Flows in Very Highly-Loaded Low-Pressure Turbine Cascades," Ph.D. thesis, Carleton University, Ottawa, Canada. 
Langston, L. S. (2001), "Secondary Flows in Axial Turbines - A Review," Annals - New York Academy of Sciences, 934, 11-26.

Langtry, R. B., and Menter, F. R. (2005) "Transition Modeling for General CFD Applications in Aeronautics," Proc. of 43rd AIAA Aerospace Sciences Meeting and Exhibit (AIAA Paper 2005-522), Reno, Nevada.

MacIsaac, G. D., Taremi, F., Knezevici, D. C., Scribner, C. A., and Sjolander, S. A. (2009), "Challenges in Using Kiel and Seven-Hole Pressure Probes in Highly Three-Dimensional Flows: Application to Turbomachinery Cascade Measurements," Proc. of 19th ISABE Conference (ISABE-2009-1209), Montreal, Canada.

MacIsaac, G. D., Sjolander, S. A., and Praisner, T. (2010), "Measurements of Losses and Reynolds Stresses in the Secondary Flow Downstream of a Low-Speed Linear Turbine Cascade,” Proc. of Turbo Expo 2010 (GT2010-22727), Glasgow, Scotland, UK.

Mahallati, A. (2003), “Aerodynamics of a Low-Pressure Turbine Airfoil under Steady and Periodically Unsteady Conditions," Ph.D. thesis, Carleton University, Ottawa, Canada.

Mee, D. J., Baines, N. C., Oldfield, M. L. G., and Dickens, T. E. (1992), “An Examination of the Contributions to Loss on a Transonic Turbine Blade in Cascade," ASME J. Turbomach., 114, $155-162$.

Menter, F. R. (1994), "Two-Equation Eddy-Viscosity Turbulence Models for Engineering Applications", AIAA Journal, 32(8), 1598-1605.

Michelassi, V., Rodi, W., and Gie , P. A. (1998), "Experimental and Numerical Investigation of Boundary-Layer and Wake Development in a Transonic Turbine Cascades," Aerospace Science and Technology, 2, 191-204.

Moffat, R. J. (1982), "Contributions to the Theory of Single-Sample Uncertainty Analysis," ASME J. Fluid Eng., 104, 250-258.

Moore, J., and Adhye, R. Y. (1985), "Secondary Flows and Losses Downstream of a Turbine Cascade,” ASME J. Eng. Gas Turbines Power, 107, 961-968.

Mukund, R., Viswanath, P. R., Narasimha, R., Prabhu, A., and Crouch, J. D. (2006), "Relaminarization in Highly Favourable Pressure Gradients on a Convex Surface," J. Fluid Mech., 566, 97-115. 
Oldfield, M. L. G., Schultz, D. L., and Nicholson, J. H. (1982), "Loss Measurements Using a Fast Traverse in an ILPT Transient Cascade," Proc. of Measuring Techniques in Transonic and Supersonic Flows in Cascades and Turbomachines.

Panchal, K., Abraham, S., Ekkad, S. V., Ng, W., Malandra, A., and Brown, B. J. (2011), "Investigation of Effect of End Wall Contouring Methods on a Transonic Turbine Blade Passage,” Proc. of Turbo Expo 2011 (GT2011-45192), Vancouver, Canada.

Pecnik, R., Pieringer, P., and Sanz, W. (2005), "Numerical Investigation of the Secondary Flow of a Transonic Turbine Stage Using Various Turbulence Closures," Proc. of Turbo Expo 2005 (GT2011-45192), Reno-Tahoe, NV, USA.

Perdichizzi, A. (1990), "Mach Number Effects on Secondary Flow Development Downstream of a Turbine Cascade,” ASME J. Turbomach., 112, 643-651.

Perdichizzi, A., Ubaldi, M., and Zunino, P. (1990), "Reynolds Stress Distribution Downstream of a Turbine Cascade,” Experimental Thermal and Fluid Science, 5, pp. 338-350.

Perdichizzi, A., and Dossena, V. (1993), "Incidence Angle and Pitch-Chord Effects on Secondary Flows Downstream of a Turbine Cascade,” ASME J. Turbomach., 115, 383-391.

Pianko, M., and Wazelt, F. (1982), "Propulsion and Energetics Panel Working Group 14 on Suitable Averaging Techniques in Non-Uniform Internal Flows," AGARD Advisory Report No. 182, Neuilly Sur Seine, France.

Popovic, I. (2005), "Measured Steady and Unsteady Aerodynamic Performance of a Family of Three Highly-Loaded Low-Pressure Turbine Cascades," Ph.D. thesis, Carleton University, Ottawa, Canada.

Popovic, I., Zhu, J., Dai, W., Sjolander, S. A., Praisner, T., and Grover, E. (2006), "Aerodynamics of a Family of Three Highly Loaded Low-Pressure Turbine Airfoils: Measured Effects of Reynolds Number and Turbulence Intensity in Steady Flow," Proc. of Turbo Expo 2006 (GT2006-91271), Barcelona, Spain.

Praisner, T. J., and Clark, J. P. (2007), "Predicting Transition in Turbomachinery - Part I: A Review and New Model Development,” ASME J. Turbomach., 129, 1-13.

Praisner, T. J., Allen-Bradley, E., Grover, E. A., Knezevici, D. C., and Sjolander, S. A. (2007), "Application of Non-Axisymmetric Endwall Contouring to Conventional and High-Lift Turbine Airfoils," Proc. of Turbo Expo 2007 (GT2007-27579), Montreal, Canada. 
Praisner, T. J., Grover, E. A., Knezevici, D. C., Popovic, I., Sjolander, S. A., Clark, J. P., and Sondergaard, R. (2008), “Toward the Expansion of Low-Pressure-Turbine Airfoil Design Space,” Proc. of Turbo Expo 2008 (GT2008-50898), Berlin, Germany.

Pullan, G., and Harvey, N. W. (2008), "The Influence of Sweep on Axial Flow Turbine Aerodynamics in the Endwall Region,” ASME J. Turbomach., 130, 041011.

Rose, M. G., Harvey, N. W., Seaman, P., Newman, D. A., and McManus, D. (2001), "Improving the Efficiency of the Trent 500 HP Turbine Using Nonaxisymmetric End Walls. Part II: Experimental Validation,” Proc. of Turbo Expo 2001 (2001-GT-0505), New Orleans, LA, USA.

Sauer, H., Muller, R., and Vogeler, K. (2001), "Reduction of Secondary Flow Losses in Turbine Cascades by Leading Edge Modifications at the Endwall," ASME J. Turbomach., 123, 207213.

Schuepbach, P., Rose, M., Gier, J., Raab, I., Germain, T., and Abhari, R. (2009), "NonAxisymmetric End Wall Profiles Including Fillet Radii, in a 1.5 Stage Axial Flow Turbine," Proc. of 8th European Conference on Turbomachinery Fluid Dynamics and Thermodynamics, Graz, Austria.

Scribner, C. A. (2011), "The Effect of Turbulence Intensity and Reynolds number on the Aerodynamic Behaviour of Kiel, Three-Hole, and Seven-Hole Pressure Probes," Master's thesis, Carleton University, Ottawa, Canada.

Sharma, O. P., and Butler, T. L. (1987), "Predictions of Endwall Losses and Secondary Flows in Axial Flow Turbine Cascades,” ASME J. Turbomach., 109, 229-236.

Sieverding, C. H., Stanislas, M., and Snoeck, J. (1980), “The Base Pressure Problem in Transonic Turbine Cascades," ASME J. Eng. Power, 102, 711-718.

Sieverding, C. H., and Wilputte, P. (1981), "Influence of Mach Number and End Wall Cooling on Secondary Flows in a Straight Nozzle Cascade,” ASME J. Eng. Power, 103, 257-263.

Sieverding, C. H. (1985), "Secondary Flows in Straight and Annular Turbine Cascades," Thermodynamics and Fluid Mechanics of Turbomachinery, 2, 621-664.

Sjolander, S. A. (2006), "MECH 5401: Turbomachinery Supplementary Course Notes," Department of Mechanical and Aerospace Engineering, Carleton University, Ottawa, ON, Canada. 
Sjolander, S. A. (2008), "Pressure and Pressure-Probe Measurements in Subsonic Wind-Tunnel Testing," Carleton University, Ottawa, Canada.

Snedden, G., Dunn, D., Von Backström, T. W., and Ingram, G. (2010), “Observations on the Selection of Objective Function for the Optimisation of Turbine Endwalls Using Computational Fluid Dynamics," Proc. of 7th South African Conference on Computational and Applied Mechanics (SACAM10), Pretoria, South Africa.

Sonoda, T., Arima, T., Olhofer, M., Sendhoff, B., Kost, F., and Giess, P. A. (2006), “A Study of Advanced High-Loaded Transonic Turbine Airfoils," ASME J. Turbomach., 128, 650-657.

Sonoda, T., Hasenjager, M., Arima, T., and Sendhoff, B. (2009), "Effect of End Wall Contouring on Performance of Ultra-Low Aspect Ratio Transonic Turbine Inlet Guide Vanes," ASME J. Turbomach., 131, 011020.

Sumner, D. (2002), “A Comparison of Data-Reduction Methods for a Seven-Hole Probe,” ASME J. Fluid Eng., 124, 523-527.

Torre, D., Vázquez, R., Blanco, E. d. 1. R., and Hodson, H. P. (2011), "A New Alternative for Reduction in Secondary Flows in Low Pressure Turbines," ASME J. Turbomach., 133, 011029 .

Vázquez, R., and Fidalgo, V. J. (2010), "The Effect of Reynolds and Mach Number on End-Wall Profiling Performance," Proc. of Turbo Expo 2010 (GT2010-22765), Glasgow, Scotland, UK.

Walsh, J. A., and Gregory-Smith, D. G. (1990), "Inlet Skew and the Growth of Secondary Losses and Vorticity in a Turbine Cascade," ASME J. Turbomach., 112, 633-642.

Wang, H. P., Olson, S. J., Goldstein, R. J., and Eckert, E. R. G. (1997), "Flow Visualization in a Linear Turbine Cascade of High Performance Turbine Blades,” ASME J. Turbomach., 119, $1-8$.

Weiss, A. P., and Fottner, L. (1995), "The Influence of Load Distribution on Secondary Flow in Straight Turbine Cascades,” ASME J. Turbomach., 117, 133-141.

Xu, L., and Denton, J. D. (1988), "The Base Pressure and Loss of a Family of Four Turbine Blades,” ASME J. Turbomach., 110, 9-17.

Yamamoto, A. (1987a), "Production and Development of Secondary Flows and Losses in Two Types of Straight Turbine Cascades: Part 1 - A Stator Case,” ASME J. Turbomach., 109, 186-193. 
Yamamoto, A. (1987b), "Production and Development of Secondary Flows and Losses in Two Types of Straight Turbine Cascades: Part 2 - A Rotor Case,” ASME J. Turbomach., 109, 194200.

Zoric, T. (2006), "Experimental Investigation of Secondary Flows in a Family of Three Highly Loaded Low-Pressure Turbine Cascades," Master's thesis, Carleton University, Ottawa, Canada.

Zoric, T., Popovic, I., Sjolander, S. A., Praisner, T., and Grover, E. (2007a), "Comparative Investigation of Three Highly Loaded LP Turbine Airfoils: Part I - Measured Profile and Secondary Losses at Design Incidence," Proc. of Turbo Expo 2007 (GT2007-27537), Montreal, Canada.

Zoric, T., Popovic, I., Sjolander, S. A., Praisner, T., and Grover, E. (2007b), "Comparative Investigation of Three Highly Loaded LP Turbine Airfoils: Part II - Measured Profile and Secondary Losses at Off-Design Incidence,” Proc. of Turbo Expo 2007 (GT2007-27538), Montreal, Canada.

Zweifel, O. (1945), "The Spacing of Turbo-Machine Blading Especially with Large Angular Deflection," Brown Boveri Review, 32, 436-444. 


\section{Appendix A}

\section{Derivation of Streamwise Vorticity}

The derivation of streamwise vorticity is presented in this section. Vorticity is defined as the curl of the velocity field:

$$
\Omega=\nabla \times V \rightarrow\left[\begin{array}{l}
\omega_{X} \\
\omega_{Y} \\
\omega_{z}
\end{array}\right]=\left[\begin{array}{l}
\frac{\partial w}{\partial y}-\frac{\partial v}{\partial z} \\
\frac{\partial u}{\partial z}-\frac{\partial w}{\partial x} \\
\frac{\partial v}{\partial x}-\frac{\partial u}{\partial y}
\end{array}\right]
$$

As shown, the axial vorticity component is determined from the velocity gradients in the pitchwise and spanwise directions, and thus it may be calculated using the measurements from a single traverse plane (e.g. $1.4 C_{X}$ plane). The other vorticity components, on the other hand, cannot be determined directly from a single traverse plane since they require velocity gradients in the axial direction as well. Alternatively, the pitchwise and spanwise pressure gradients may be used to obtain the components of vorticity, by employing a few simplifying assumptions as described by Perdichizzi (1990) and Yaras (1990). Assuming inviscid flow, the Crocco equation for steady, compressible flow is expressed as:

$$
V \times \Omega=\nabla h_{0}-T \nabla s
$$

and assuming adiabatic flow:

$$
V \times \Omega=-T \nabla s \rightarrow\left[\begin{array}{l}
v \omega_{Z}-w \omega_{Y} \\
w \omega_{X}-u \omega_{Z} \\
u \omega_{Y}-v \omega_{X}
\end{array}\right]=-T\left[\begin{array}{l}
\frac{\partial s}{\partial x} \\
\frac{\partial s}{\partial y} \\
\frac{\partial s}{\partial z}
\end{array}\right]
$$

Next, applying the second law for an adiabatic system: 


$$
\boldsymbol{d} \boldsymbol{s}=-\boldsymbol{R} \boldsymbol{d}\left(\ln P_{0}\right) \rightarrow\left[\begin{array}{l}
\boldsymbol{v} \omega_{z}-w \omega_{Y} \\
\boldsymbol{w} \omega_{X}-\boldsymbol{u} \omega_{z} \\
\boldsymbol{u} \omega_{Y}-\boldsymbol{v} \omega_{X}
\end{array}\right]=R T\left[\begin{array}{l}
\frac{\partial\left(\ln P_{0}\right)}{\partial x} \\
\frac{\partial\left(\ln P_{0}\right)}{\partial y} \\
\frac{\partial\left(\ln P_{0}\right)}{\partial z}
\end{array}\right]=\frac{a^{2}}{\gamma}\left[\begin{array}{l}
\frac{\partial\left(\ln P_{0}\right)}{\partial x} \\
\frac{\partial\left(\ln P_{0}\right)}{\partial y} \\
\frac{\partial\left(\ln P_{0}\right)}{\partial z}
\end{array}\right]
$$

The pitchwise and spanwise vorticity components are determined as follows:

$$
\begin{aligned}
& \omega_{Y}=\frac{1}{u}\left(v \omega_{X}+\frac{a^{2}}{\gamma} \frac{\partial\left(\ln P_{0}\right)}{\partial z}\right) \\
& \omega_{Z}=\frac{1}{u}\left(w \omega_{X}-\frac{a^{2}}{\gamma} \frac{\partial\left(\ln P_{0}\right)}{\partial y}\right)
\end{aligned}
$$

Gregory-Smith et al. (1998) and Yaras (1998) employed the incompressible Euler equation, expressed in the Helmholtz form, to determine the pitchwise and spanwise vorticity components. They also examined the effects of viscosity on the derived vorticity components, and concluded that apart from small differences near the vortex core, the results were in generally good agreement. The applicability of the inviscid assumption in Crocco's relation for the compressible flow field in the present study was examined using the CFD results. The vorticity components were computed using both methods, Equation A.1 and Equation A.5, and found to be very similar.

The component of vorticity in the streamwise direction (profile flow direction) is obtained from the axial and pitchwise components of vorticity as shown in Figure A.1. The spanwise vorticity component is not required here since it is perpendicular to the profile flow direction.
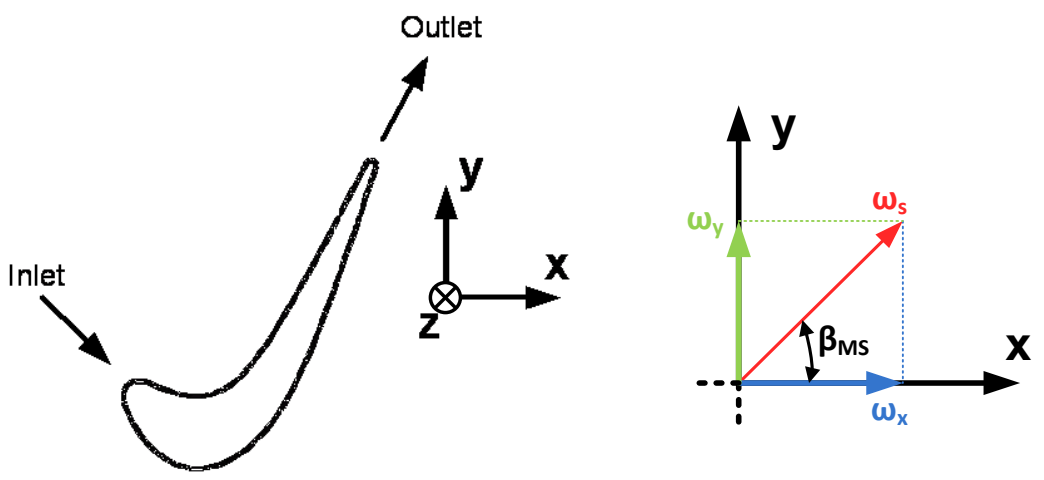

Figure A.1 Cascade coordinate system and vorticity components 


$$
\omega_{S}=\omega_{X} \cos \beta_{M S}+\omega_{Y} \sin \beta_{M S}
$$

Finally, the non-dimensional streamwise vorticity coefficient is defined as:

$$
\boldsymbol{C} \omega_{S}=\frac{\boldsymbol{C}}{V_{2, i s}}\left(\omega_{X} \cos \beta_{M S}+\omega_{Y} \sin \beta_{M S}\right)
$$




\section{Appendix B}

\section{Pitchwise Averaged Plots}

The pitchwise mass-averaged results both presented in Chapters 7 through 9. The differences between pitchwise mass-averaging and pitchwise area-averaging are described here. The pitchwise area-averaged results are determined using Equation B.1:

$$
\phi_{\text {area }}^{\prime}=\int_{0}^{1} \phi d(y / s)
$$

Therefore, the pitchwise area-averaged plots may be visually integrated (e.g. by the reader) to estimate the overall area-averaged value. A different situation occurs when

processing the pitchwise mass-averaged plots. Initially, the pitchwise mass-averaged plots were generated using Equation B.2:

$$
\phi_{\text {mass }}^{\prime}=\frac{\int_{0}^{1}(\rho u) \phi d(y / s)}{\int_{0}^{1}(\rho u) d(y / s)} \quad(\text { Method } 1)
$$

If this parameter is plotted in terms of the spanwise location $(z / h)$, the area under the curve is no longer representative of the overall mass-averaged value; the area under the curve represents a combination of mass-averaging in the pitchwise direction and areaaveraging in the spanwise direction since it does not account for any spanwise variations in mass flux. In view of this discrepancy, the pitchwise mass-averaged results are determined in two steps: the results are first weighted based on the mass flux ratio, $\rho u_{\text {local }} \rho u_{\text {total }}$, and then plotted using Equation B.3:

$$
\phi_{\text {mass }}^{\prime}=\frac{(\rho u)_{\text {local }}}{(\rho u)_{\text {total }}} \cdot \frac{\int_{0}^{1}(\rho u) \phi d(y / s)}{\int_{0}^{1}(\rho u) d(y / s)} \quad \text { (Method 2) }
$$

Here, $\rho u_{\text {local }}$ represents the mass flux at the particular spanwise location, and $\rho u_{\text {total }}$ represents the total integrated mass flow over the traverse plane. 
Consequently, the area under the curve based on Equation B.3 is representative of the overall mass-averaged value, both in the pitchwise and spanwise directions.

An example of the different pitchwise mass-averaging methods is shown in Figure B.1 based on the SL4F results (Figure B.2). Here, the pitch-averaged plot based on Equation B.2 results in the amplification of losses at approximately $15 \%$ span due to higher mass fluxes, and the reduction of near-wall losses $(3 \%<z / h<7 \%)$ due to lower mass fluxes. This effect is consistent with the overall mass-averaging procedure.

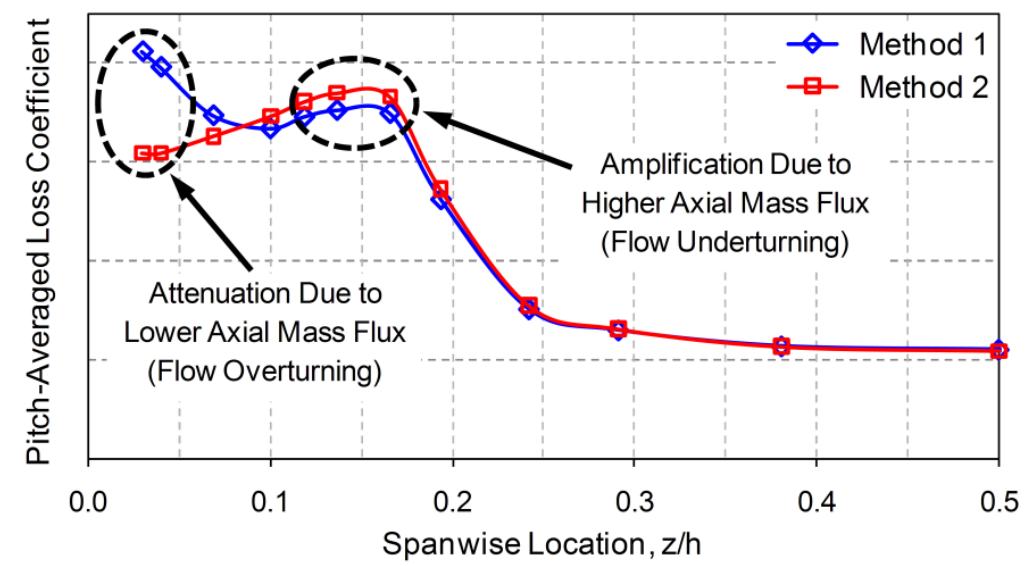

Figure B.1 Comparison of the two methods for the presentation of the pitchwise massaveraged loss coefficients

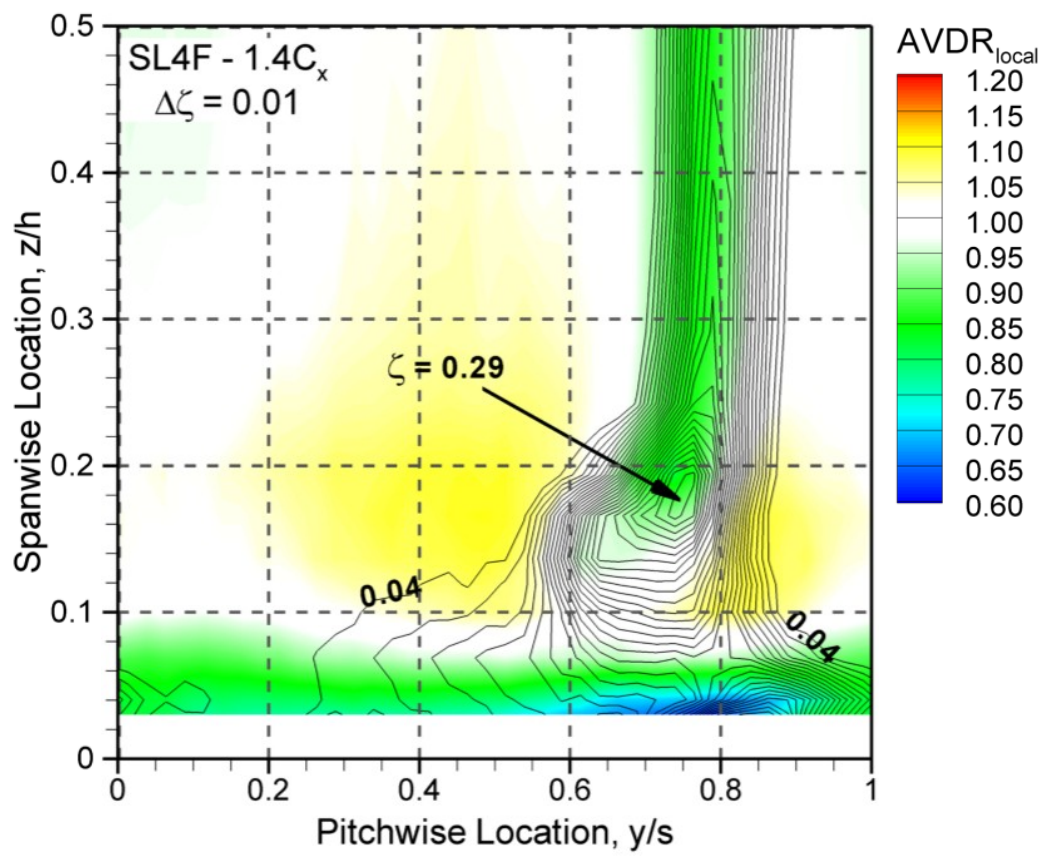

Figure B.2 Colour floods of local axial velocity density ratio superimposed with contour lines of energy loss coefficient 


\section{Appendix C}

\section{Loss Mixing Out Procedure}

This section describes the loss mixing-out procedures developed by Oldfield et al. (1981) and Amecke and Safarik (1995). The method is applicable to the threedimensional, compressible flow field downstream of the cascade. The conservation of mass and momentum equations are solved simultaneously to determine the mixed-out flow parameters from the collected data at the downstream traverse planes (e.g. $1.4 C_{X}$ ). The conservation of energy is not required here since total temperature variation downstream of the cascade is assumed negligible ( $T_{0}$ is constant).

In the following derivation, the subscripts " 1 ", " 2 " and " $\mathrm{m}$ " refer to the inlet plane, the exit plane (data collection location) and the mixed-out plane, respectively. Furthermore, the limits of integration are set to the following values corresponding to the experimental boundaries:

- Pitchwise direction $(y / s): 0$ to 1 (covering one blade pitch)

- $\quad$ Spanwise direction $(z / h): 0$ to 0.5 (from the endwall to midspan)

As shown in Figure C.1, the conservation equations are set up between the measurement plane and the mixed-out plane, assuming frictionless parallel walls:

$$
\begin{aligned}
& \text { Conservation of mass } \rightarrow \rho_{m} u_{m}=\frac{1}{0.5} \int_{0}^{0.5} \int_{0}^{1} \rho_{2} u_{2} d(y / s) d(z / h) \\
& \rho_{m} U_{m} \frac{1}{\sqrt{1+\tan ^{2} \alpha_{m}+\tan ^{2} \beta_{m}}}=2 \int_{0}^{0.5} \int_{0}^{1} \rho_{2} U_{2} \frac{1}{\sqrt{1+\tan ^{2} \alpha_{2}+\tan ^{2} \beta_{2}}} d(y / s) d(z / h) \\
& \text { Conservation of x-momentum } \rightarrow \rho_{m} u_{m}^{2}+P_{m}=\frac{1}{0.5} \int_{0}^{0.5} \int_{0}^{1}\left(\rho_{2} u_{2}^{2}+P_{2}\right) d(y / s) d(z / h) \\
& \rho_{m} U_{m}^{2} \frac{1}{1+\tan ^{2} \alpha_{m}+\tan ^{2} \beta_{m}}+P_{m}=2 \int_{0}^{0.5} \int_{0}^{1}\left(\rho_{2} U_{2}^{2} \frac{1}{1+\tan ^{2} \alpha_{2}+\tan ^{2} \beta_{2}}+P_{2}\right) d(y / s) d(z / h)
\end{aligned}
$$




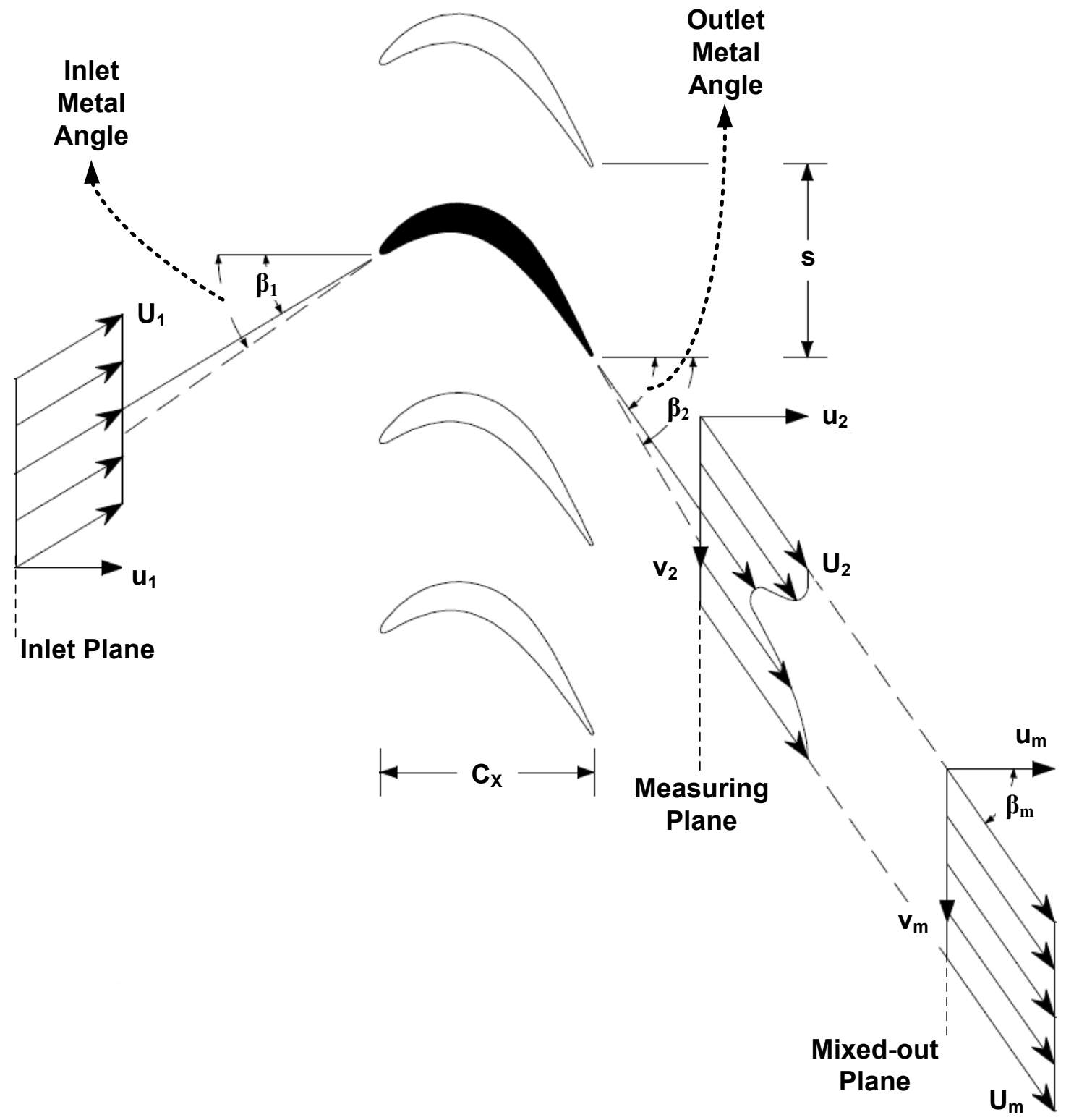

Figure C.1 Loss mixing-out domain (reproduced from Mahallati, 2003)

Conservation of y-momentum $\rightarrow \rho_{m} u_{m} v_{m}=\frac{1}{0.5} \int_{0}^{0.5} \int_{0}^{1} \rho_{2} u_{2} v_{2} d(y / s) d(z / h)$

$\rho_{m} U_{m}^{2} \frac{\tan \beta_{m}}{1+\tan ^{2} \alpha_{m}+\tan ^{2} \beta_{m}}=2 \int_{0}^{0.5} \int_{0}^{1}\left(\rho_{2} U_{2}^{2} \frac{\tan \beta_{2}}{1+\tan ^{2} \alpha_{2}+\tan ^{2} \beta_{2}}\right) d(y / s) d(z / h)$

Conservation of z-momentum $\rightarrow \rho_{m} u_{m} w_{m}=\frac{1}{0.5} \int_{0}^{0.5} \int_{0}^{1} \rho_{2} u_{2} w_{2} d(y / s) d(z / h)$

$\rho_{m} U_{m}^{2} \frac{\tan \alpha_{m}}{1+\tan ^{2} \alpha_{m}+\tan ^{2} \beta_{m}}=2 \int_{0}^{0.5} \int_{0}^{1}\left(\rho_{2} U_{2}^{2} \frac{\tan \alpha_{2}}{1+\tan ^{2} \alpha_{2}+\tan ^{2} \beta_{2}}\right) d(y / s) d(z / h)$ 
The equations are further rearranged to express the results in terms of the four fundamental parameters: $P_{0}, P, \alpha$ and $\beta$. Furthermore, the critical mass flow rate is used to non-dimensionalized the equations as follows:

$$
\Theta=\frac{\rho \cdot U}{\rho^{*} \cdot a^{*}}=\left(\frac{P}{P_{0}}\right)^{\frac{1}{\gamma}}\left(\sqrt{1-\left(\frac{P}{P_{0}}\right)^{\frac{\gamma-1}{\gamma}}}\right)\left(\sqrt{\left(\frac{2}{\gamma-1}\right)\left(\frac{\gamma+1}{2}\right)^{\frac{\gamma+1}{\gamma-1}}}\right)
$$

Normalizing Equation $\mathrm{C} 1$ by $\rho_{1}^{*} a_{1}^{*}$ :

$$
\begin{gathered}
\frac{\rho_{m}^{*} a_{m}^{*}}{\rho_{1}^{*} a_{1}^{*}} \frac{\Theta_{m}}{\sqrt{1+\tan ^{2} \alpha_{m}+\tan ^{2} \beta_{m}}}=2 \int_{0}^{0.5} \int_{0}^{1} \frac{\rho_{2}^{*} a_{2}^{*}}{\rho_{1}^{*} a_{1}^{*}} \frac{\Theta_{2}}{\sqrt{1+\tan ^{2} \alpha_{2}+\tan ^{2} \beta_{2}}} d\left(\frac{P_{m}}{P_{0 m}}\right)^{\frac{1}{\gamma}}\left(\sqrt{1-\left(\frac{P_{m}}{P_{0 m}}\right)^{\frac{\gamma-1}{\gamma}}}\right)\left(\sqrt{\left(\frac{2}{\gamma-1}\right)\left(\frac{\gamma+1}{2}\right)^{\frac{\gamma+1}{\gamma-1}}}\right) \\
\sqrt{1+\tan ^{2} \alpha_{m}+\tan ^{2} \beta_{m}}
\end{gathered}
$$

Next, normalizing Equations $\mathrm{C} 2, \mathrm{C} 3$ and $\mathrm{C} 4$ by $\mathrm{P}_{01}$ :

$$
\begin{aligned}
\frac{P_{0 m}}{P_{01}}\left\{\left[\frac{\gamma}{\gamma-1}\left(\frac{P_{m}}{P_{0 m}}\right)^{\frac{1}{\gamma}}\left(1-\left(\frac{P_{m}}{P_{0 m}}\right)^{\frac{\gamma-1}{\gamma}}\right)\right] \frac{2}{1+\tan ^{2} \alpha_{m}+\tan ^{2} \beta_{m}}+\frac{P_{m}}{P_{0 m}}\right\}= \\
\quad 2 \int_{0}^{0.5} \int_{0}^{1} \frac{P_{02}}{P_{01}}\left\{\left[\frac{\gamma}{\gamma-1}\left(\frac{P_{2}}{P_{02}}\right)^{\frac{1}{\gamma}}\left(1-\left(\frac{P_{2}}{P_{02}}\right)^{\frac{\gamma-1}{\gamma}}\right)\right] \frac{2}{1+\tan ^{2} \alpha_{2}+\tan ^{2} \beta_{2}}+\frac{P_{2}}{P_{02}}\right\} d\left(\frac{y}{s}\right) d\left(\frac{z}{h}\right) \\
\frac{P_{0 m}}{P_{01}}\left\{\left[\frac{\gamma}{\gamma-1}\left(\frac{P_{m}}{P_{0 m}}\right)^{\frac{1}{\gamma}}\left(1-\left(\frac{P_{m}}{P_{0 m}}\right)^{\frac{\gamma-1}{\gamma}}\right)\right] \frac{2 \cdot \tan \beta_{m}}{1+\tan ^{2} \alpha_{m}+\tan ^{2} \beta_{m}}\right\}= \\
\quad 2 \int_{0}^{0.5} \int_{0}^{1} \frac{P_{02}}{P_{01}}\left\{\left[\frac{\gamma}{\gamma-1}\left(\frac{P_{2}}{P_{02}}\right)^{\frac{1}{\gamma}}\left(1-\left(\frac{P_{2}}{P_{02}}\right)^{\frac{\gamma-1}{\gamma}}\right)\right] \frac{2 \cdot \tan \beta_{2}}{1+\tan ^{2} \alpha_{2}+\tan ^{2} \beta_{2}}\right\} d\left(\frac{y}{s}\right) d\left(\frac{z}{h}\right)
\end{aligned}
$$




$$
\begin{aligned}
& \frac{P_{0 m}}{P_{01}}\left\{\left[\frac{\gamma}{\gamma-1}\left(\frac{P_{m}}{P_{0 m}}\right)^{\frac{1}{\gamma}}\left(1-\left(\frac{P_{m}}{P_{0 m}}\right)^{\frac{\gamma-1}{\gamma}}\right)\right] \frac{2 \cdot \tan \alpha_{m}}{1+\tan ^{2} \alpha_{m}+\tan ^{2} \beta_{m}}\right\}= \\
& \quad 2 \int_{0}^{0.5} \int_{0}^{1} \frac{P_{02}}{P_{01}}\left\{\left[\frac{\gamma}{\gamma-1}\left(\frac{P_{2}}{P_{02}}\right)^{\frac{1}{\gamma}}\left(1-\left(\frac{P_{2}}{P_{02}}\right)^{\frac{\gamma-1}{\gamma}}\right)\right] \frac{2 \cdot \tan \alpha_{2}}{1+\tan ^{2} \alpha_{2}+\tan ^{2} \beta_{2}}\right\} d\left(\frac{y}{s}\right) d\left(\frac{z}{h}\right)
\end{aligned}
$$

Assigning: $\quad K=\frac{P_{2}}{P_{02}}$

$$
\frac{P_{0 m}}{P_{01}} \frac{\left(\frac{P_{m}}{P_{0 m}}\right)^{\frac{1}{\gamma}}\left(\sqrt{1-\left(\frac{P_{m}}{P_{0 m}}\right)^{\frac{\gamma-1}{\gamma}}}\right)\left(\sqrt{\left(\frac{2}{\gamma-1}\right)\left(\frac{\gamma+1}{2}\right)^{\frac{\gamma+1}{\gamma-1}}}\right)}{\sqrt{1+\tan ^{2} \alpha_{m}+\tan ^{2} \beta_{m}}}=
$$

$$
2 \int_{0}^{0.5} \int_{0}^{1} \frac{P_{02} \frac{\sqrt{K^{\frac{2}{\gamma}}-K^{\frac{\gamma+1}{\gamma}}}}{P_{01}} \sqrt{\left(\frac{2}{\gamma-1}\right)\left(\frac{\gamma+1}{2}\right)^{\frac{\gamma+1}{\gamma-1}}}}{\sqrt{1+\tan ^{2} \alpha_{2}+\tan ^{2} \beta_{2}}} d\left(\frac{y}{s}\right) d\left(\frac{z}{h}\right)
$$

$$
\frac{P_{0 m}}{P_{01}}\left\{\left[\frac{\gamma}{\gamma-1}\left(\frac{P_{m}}{P_{0 m}}\right)^{\frac{1}{\gamma}}\left(1-\left(\frac{P_{m}}{P_{0 m}}\right)^{\frac{\gamma-1}{\gamma}}\right)\right] \frac{2}{1+\tan ^{2} \alpha_{m}+\tan ^{2} \beta_{m}}+\frac{P_{m}}{P_{0 m}}\right\}=
$$

$$
2 \int_{0}^{0.5} \int_{0}^{1} \frac{P_{02}}{P_{01}}\left\{\left(\frac{\gamma}{\gamma-1}\right)\left(K^{\frac{1}{\gamma}}-K\right) \frac{2}{1+\tan ^{2} \alpha_{2}+\tan ^{2} \beta_{2}}+K\right\} d\left(\frac{y}{s}\right) d\left(\frac{z}{h}\right)
$$

$$
\frac{P_{0 m}}{P_{01}}\left\{\left[\frac{\gamma}{\gamma-1}\left(\frac{P_{m}}{P_{0 m}}\right)^{\frac{1}{\gamma}}\left(1-\left(\frac{P_{m}}{P_{0 m}}\right)^{\frac{\gamma-1}{\gamma}}\right)\right] \frac{2 \cdot \tan \beta_{m}}{1+\tan ^{2} \alpha_{m}+\tan ^{2} \beta_{m}}\right\}=
$$

$$
2 \int_{0}^{0.5} \int_{0}^{1} \frac{P_{02}}{P_{01}}\left\{\left(\frac{\gamma}{\gamma-1}\right)\left(K^{\frac{1}{\gamma}}-K\right) \frac{2 \cdot \tan \beta_{2}}{1+\tan ^{2} \alpha_{2}+\tan ^{2} \beta_{2}}\right\} d\left(\frac{y}{s}\right) d\left(\frac{z}{h}\right)
$$

$$
\frac{P_{0 m}}{P_{01}}\left\{\left[\frac{\gamma}{\gamma-1}\left(\frac{P_{m}}{P_{0 m}}\right)^{\frac{1}{\gamma}}\left(1-\left(\frac{P_{m}}{P_{0 m}}\right)^{\frac{\gamma-1}{\gamma}}\right)\right] \frac{2 \cdot \tan \alpha_{m}}{1+\tan ^{2} \alpha_{m}+\tan ^{2} \beta_{m}}\right\}=
$$

$$
2 \int_{0}^{0.5} \int_{0}^{1} \frac{P_{02}}{P_{01}}\left\{\left(\frac{\gamma}{\gamma-1}\right)\left(K^{\frac{1}{\gamma}}-K\right) \frac{2 \cdot \tan \alpha_{2}}{1+\tan ^{2} \alpha_{2}+\tan ^{2} \beta_{2}}\right\} d\left(\frac{y}{s}\right) d\left(\frac{z}{h}\right)
$$


The integrated parameters on the R.H.S. of the equations $\left(I_{M}, I_{X}, I_{Y}\right.$ and $\left.I_{Z}\right)$ are evaluated based on the data from the traverse plane (e.g. $1.4 C_{X}$ ). The four equations are solved simultaneously using a MATLAB script to obtain the mixed-out parameters of interest: total and static pressures, and pitchwise and spanwise flow angles. Other mixedout parameters, such as Mach number and loss coefficients, are determined based on these. 